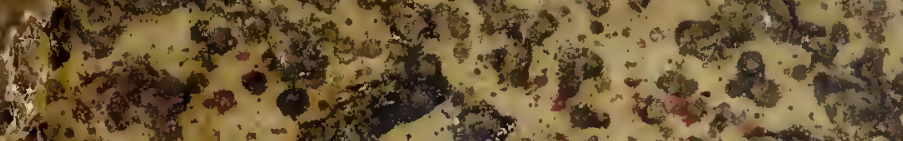

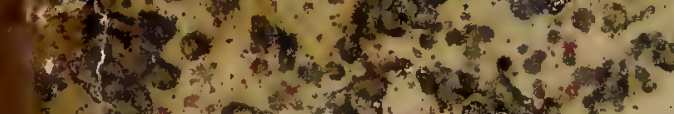

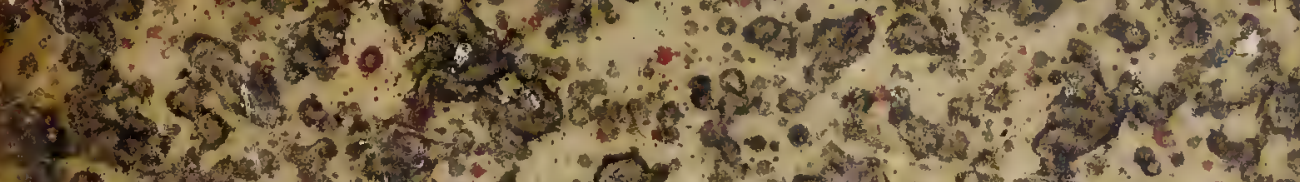

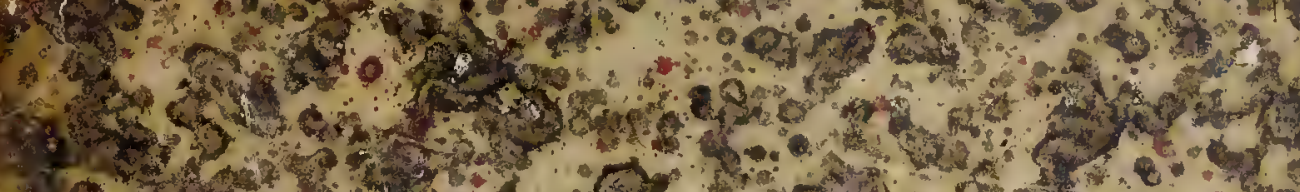

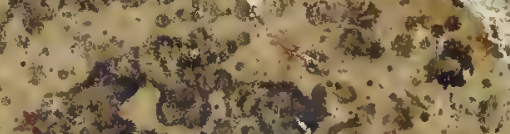

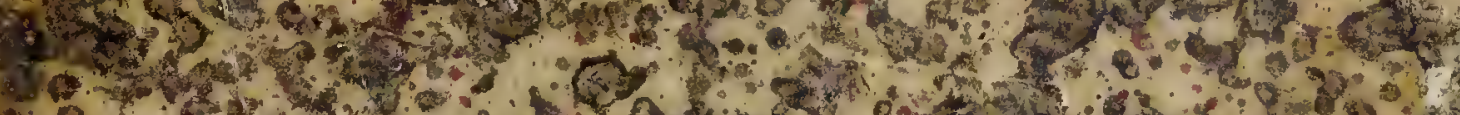

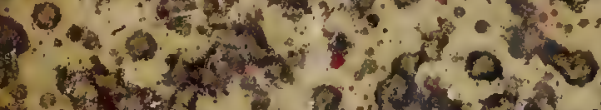

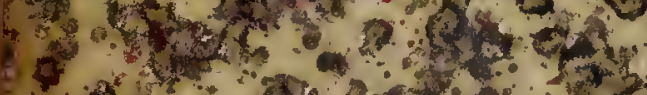

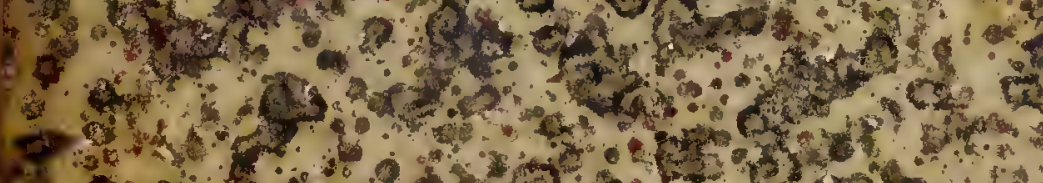

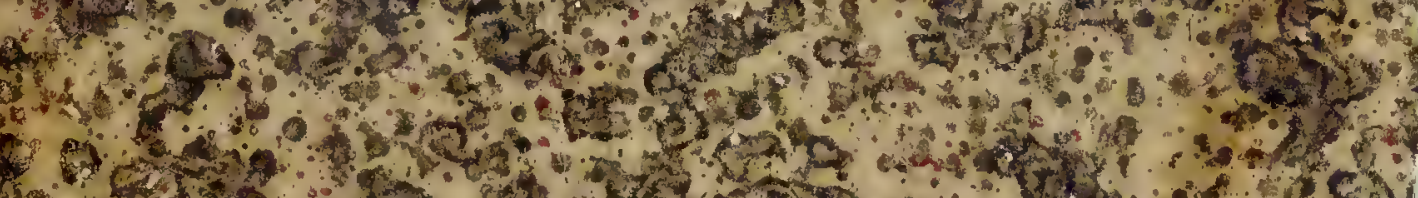

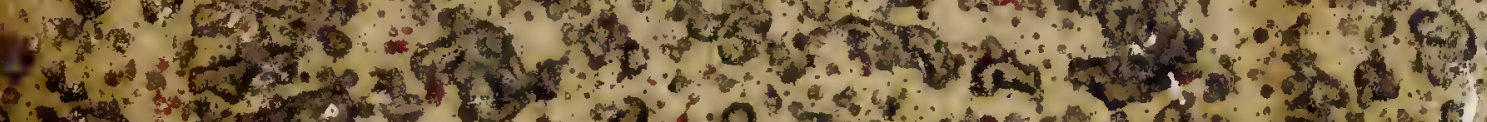

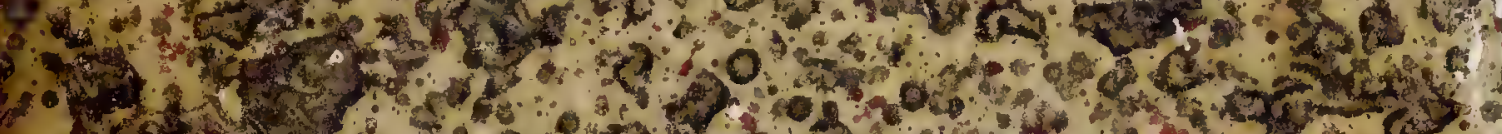

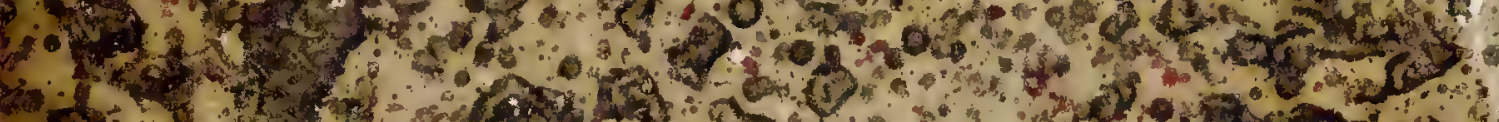

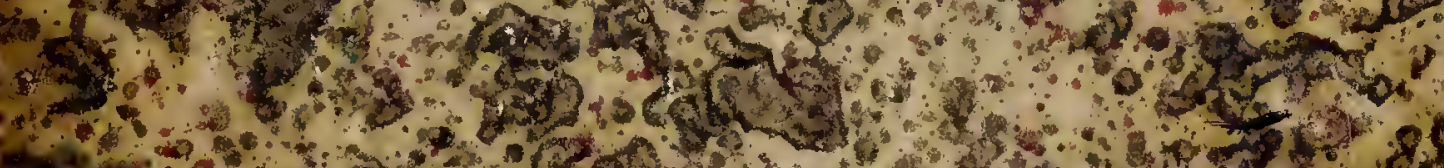

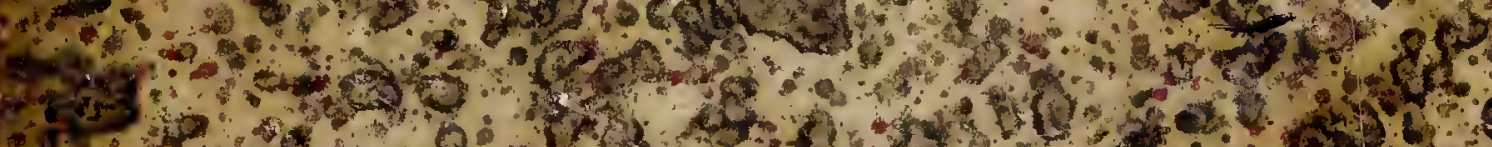

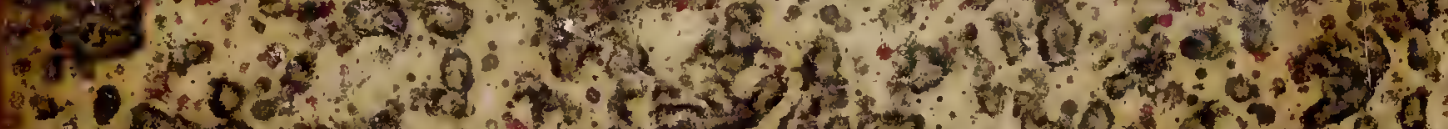

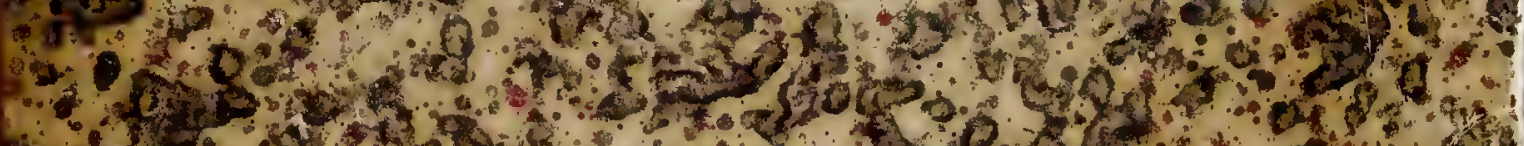

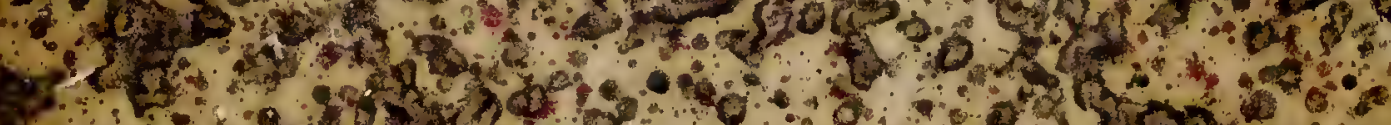

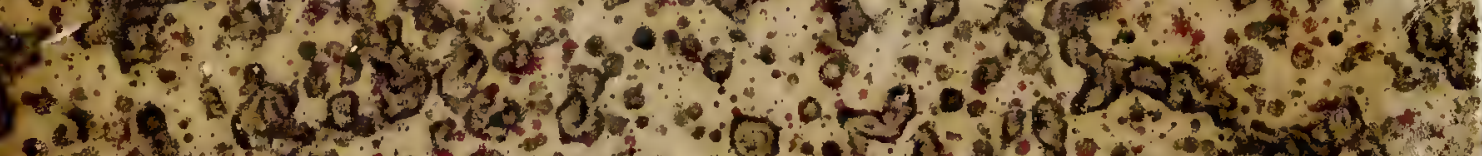

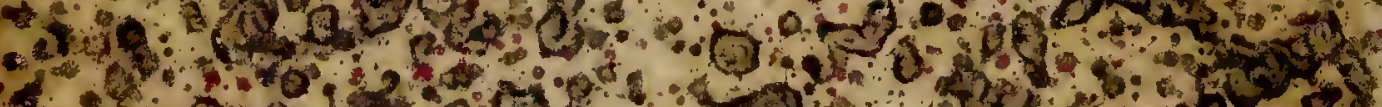

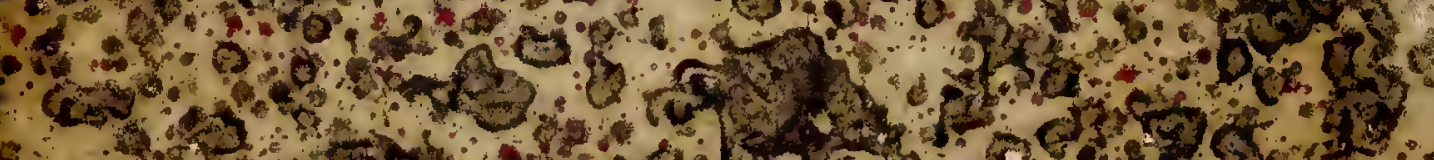

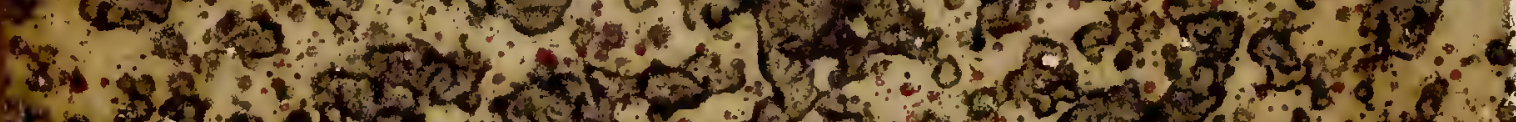

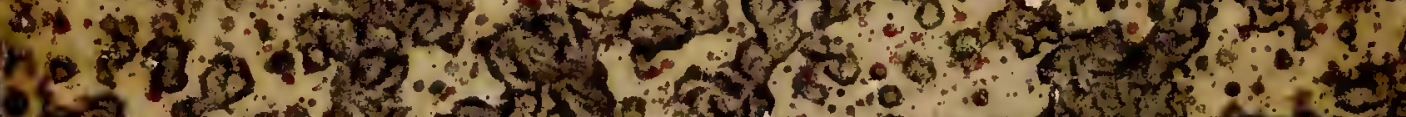

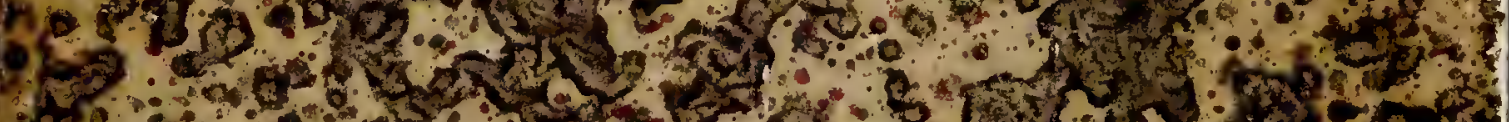

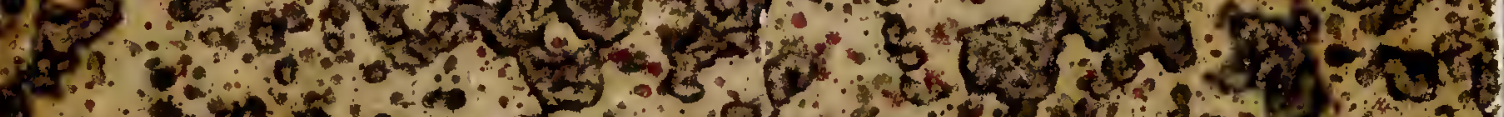

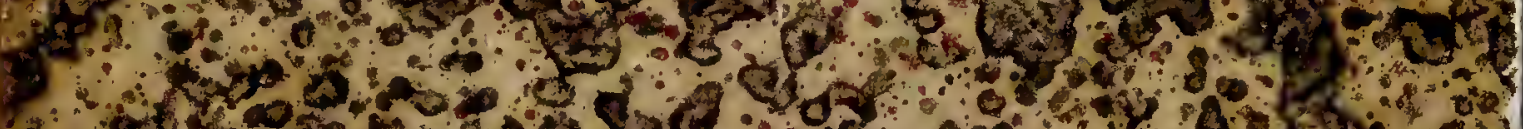

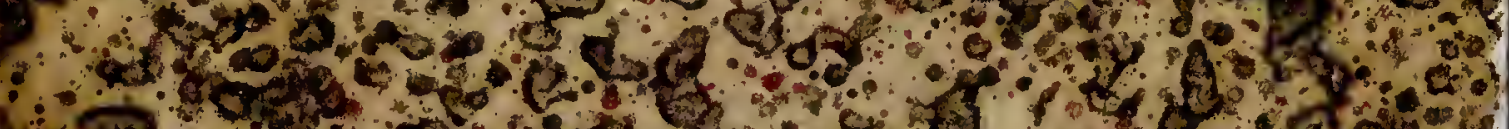
$\therefore$ :

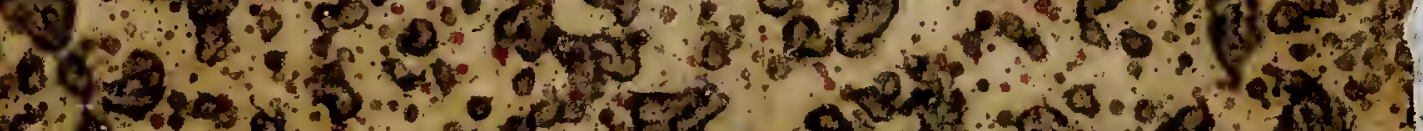

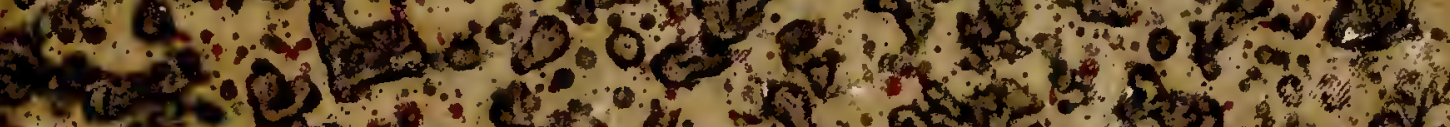

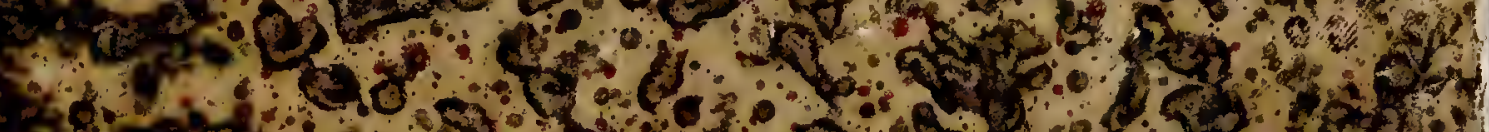

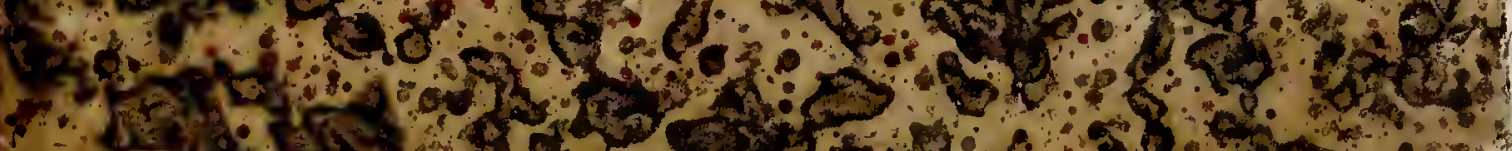

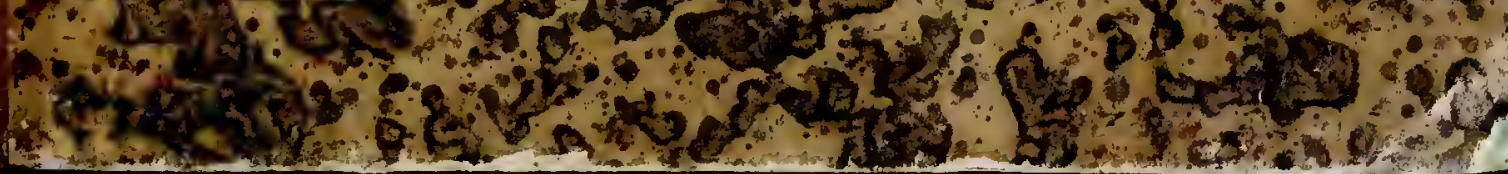


1)
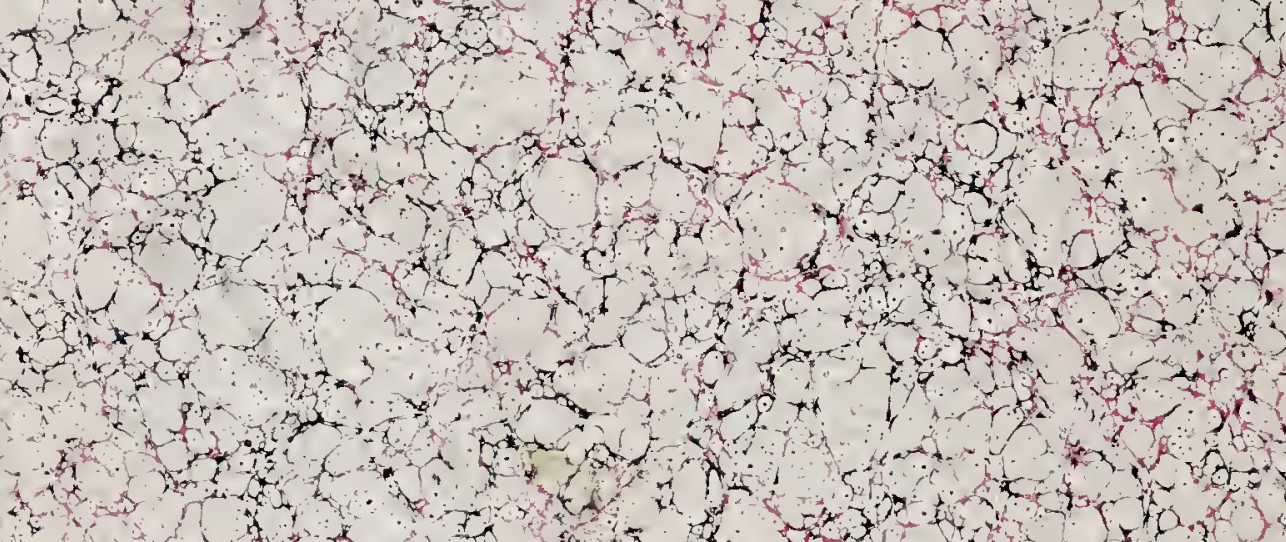

$21 x^{2}$

$$
\text { 1. }+4+1
$$

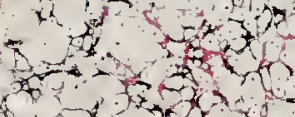

se

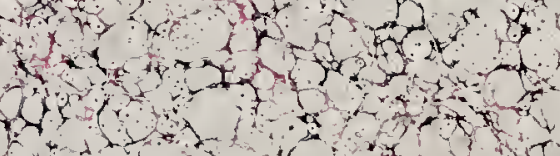

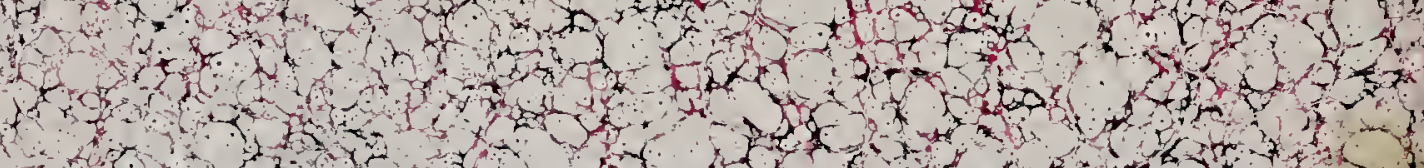
(1)

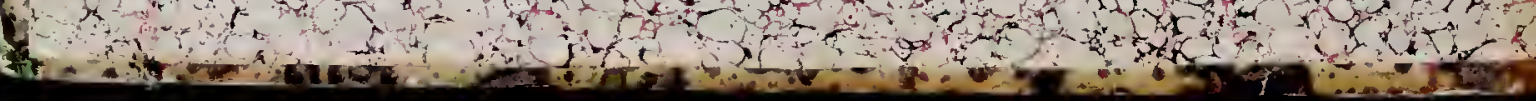




$$
\begin{aligned}
& 7+x+2
\end{aligned}
$$

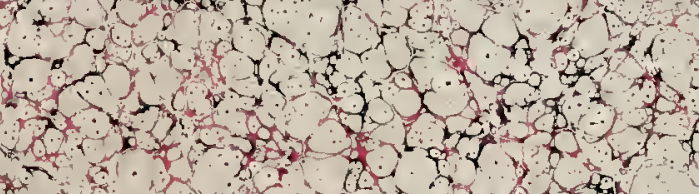

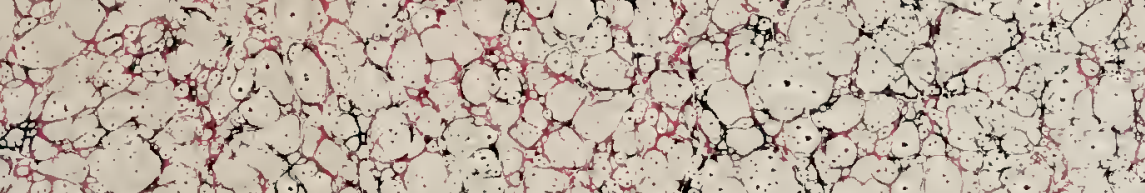

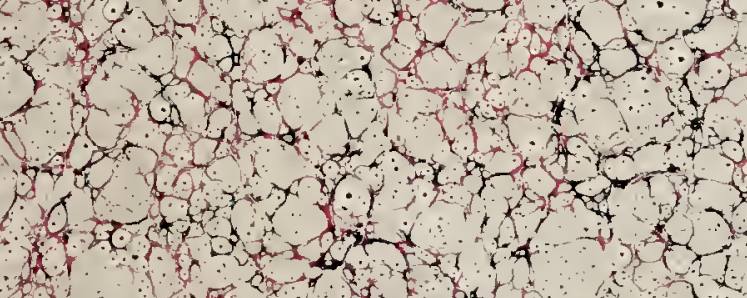

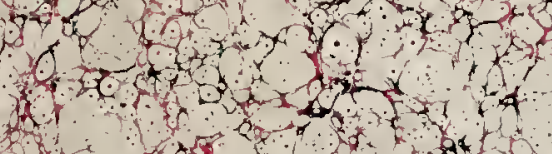

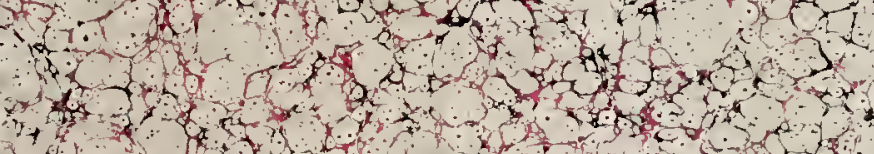

tenting

$x+2, x+1$

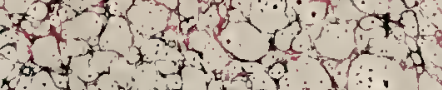

$x+7 \times+4$

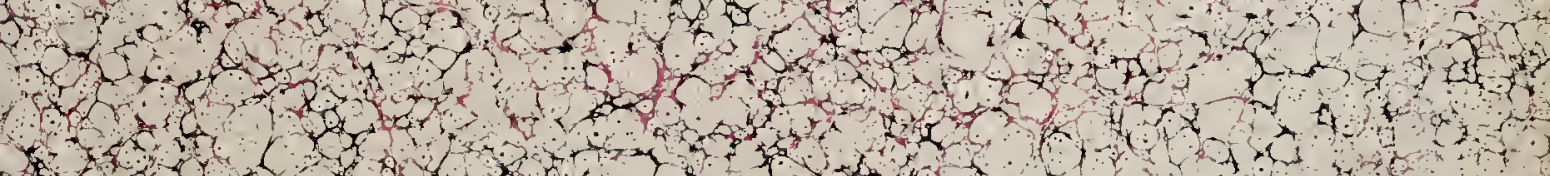

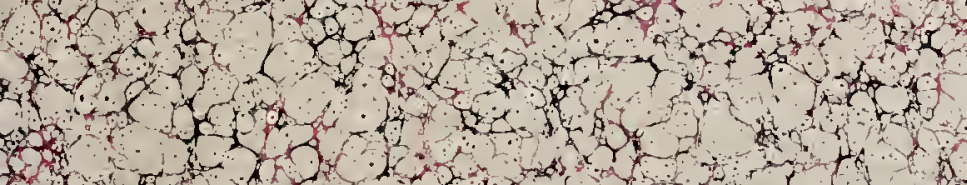

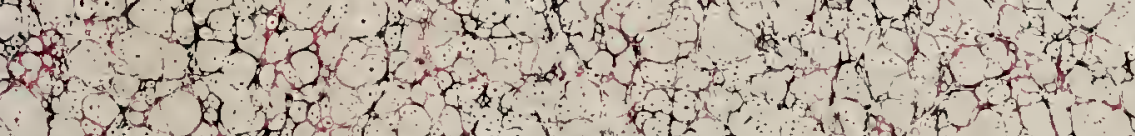

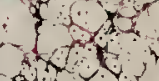

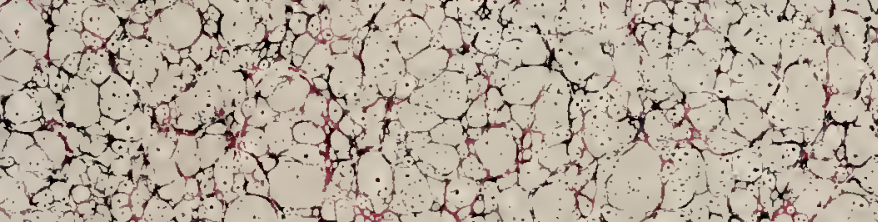

ro.t.

1.

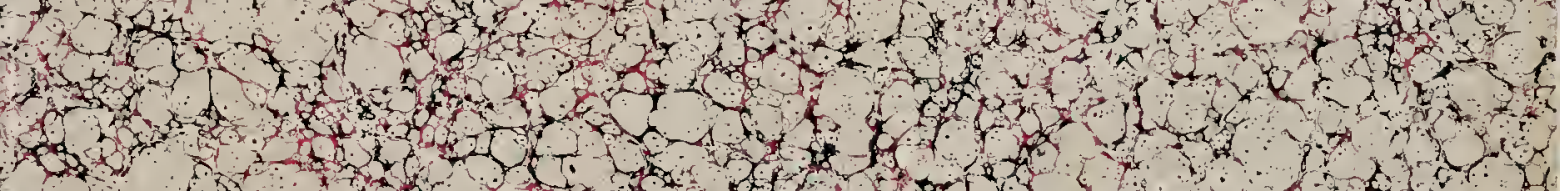

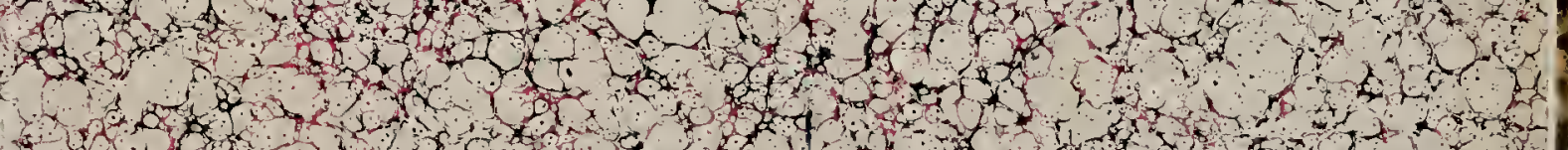

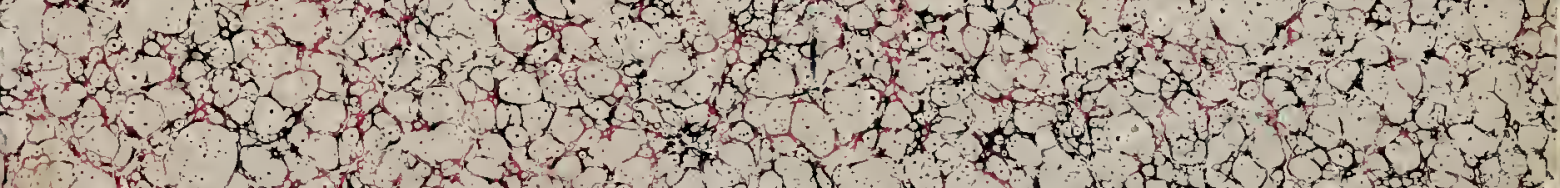

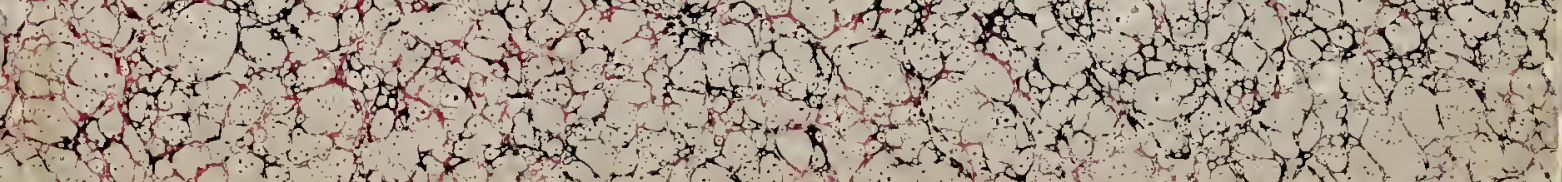




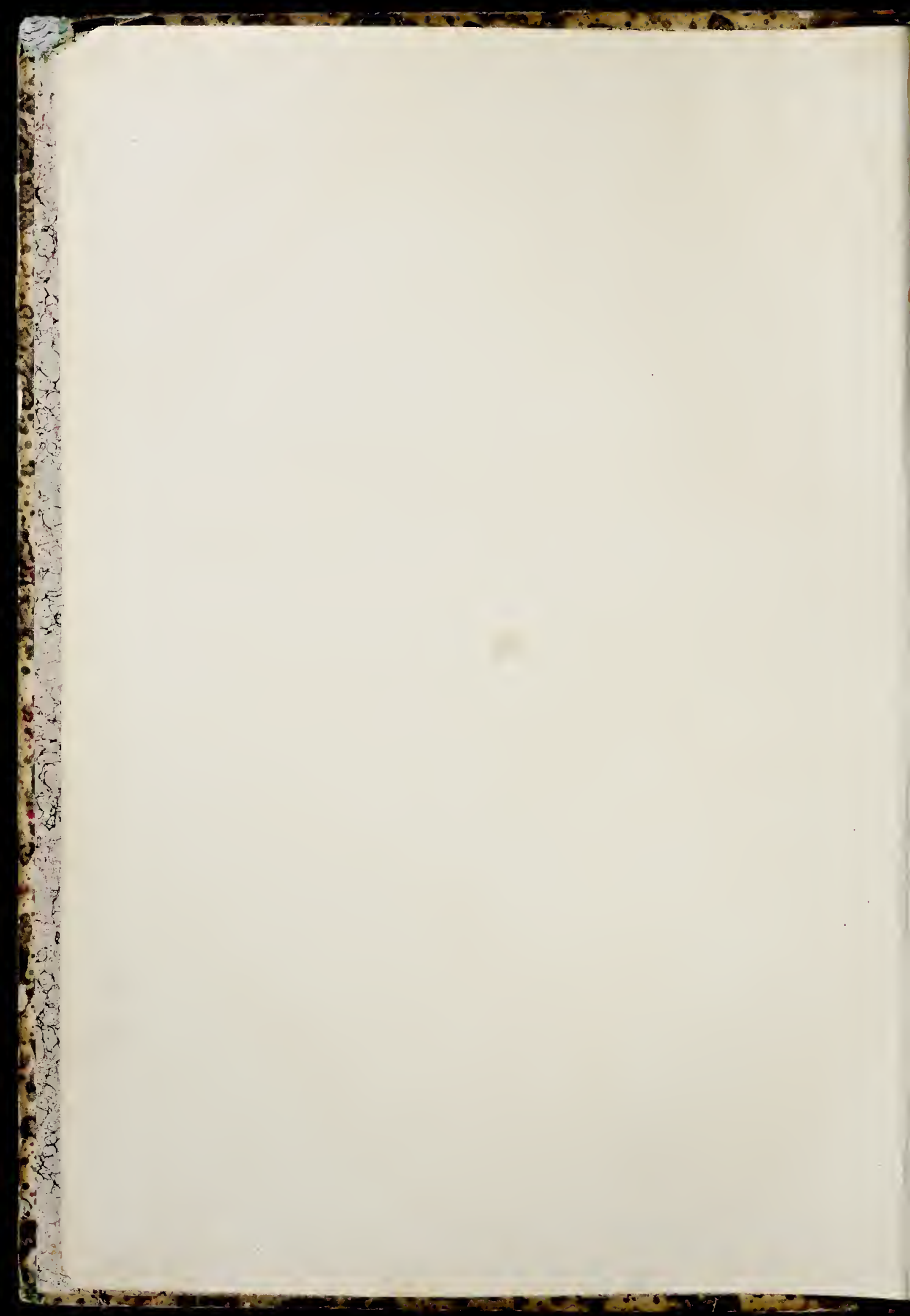





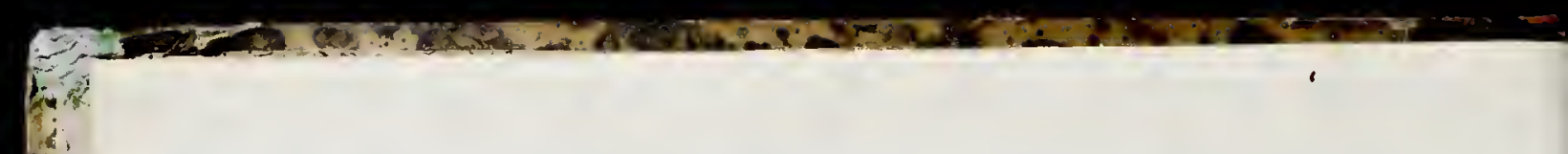




\section{plans mainoniés}

1)l:

\section{TOUTES LES ESPECES DE JADDINS,}

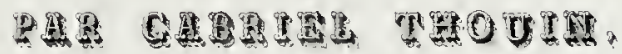

\section{Croisimi gefition.}

\section{(1)}

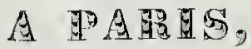

CHEZ MADAME hUZARD ( NE VALdAT LA CHAPELLE), IMPRIMEUR - LIBRAIRE, RUE DE Y'T́PERON-SALNT-ANDTÉ-DES-ARTS, No 7 . 


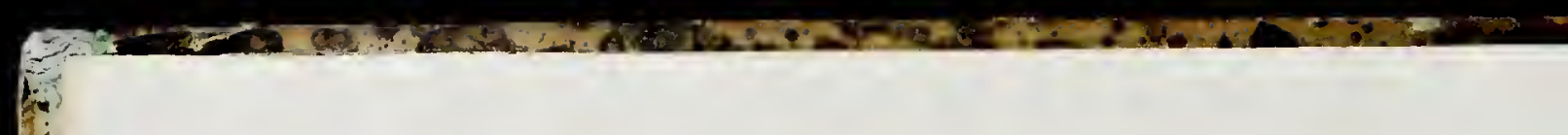




\section{AVIS DE LEOITEUR}

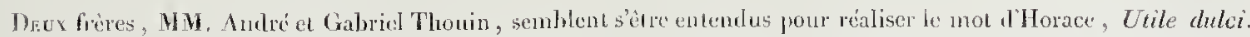

Dans son Conrs de Culture (1), fruit de soixante ans de travaux, de lecons publiques au Jarelin du Roi, d'applicalions continnellement réitírées, d'essais faits avec toutes les modifications que sa position lui permettait et que sa passion pour ce genle d'étule pouvait lui suggérer, M. Iudré Thouin a posé des principes, tracé ıles règles d'une utitite incontestable, et qui forment une sorte de corle de la Nature el de I'Art, où clargue genre de culture est latisonné, drimontré, approfondi

A eốté de ce monument élevé ì la science de I'Tlorticulture, M. Gabriel Thonin a dessiné, dans son Recueil te Plans de Jardins, l'ensemble et les détails dont se constituent nos Jardins d'ingrement. Si son frère analyse la terre ‘fui conviont à telle essence végétale, au potager, au légumier, au verger, M. Gabriel décrit les emplacemens que l'art doit lent assigner. L'art consiste daus l'mitation de la betle Nature, et le beau, en ce genre, exige I'adroiles combinaisons, qui présentent à la vue, sous les formes les plus variées, ici des tapis de gazon, lis des bostuets, aillerrs une verdure émaillée de fleurs, plus loin une ombre produite par une épaisse feuillée. Un systeune me préscnte-t-il des allées droites, symétriquement alignées sur un plan horizontal, un autré me plait par la flexibilité des délours sinueus de ses pronenades, par ces rampes adoncies qui m'évent inseusiblement sur uno collinx pittoresque, on m'entraînent, par une pente artistement tracée, dans un vallon enchanté, sur le bord d'un Inissean, d'un bassia, d'un lac, dont une main ingénieuse a su cmbellir le pourtour et orner l'intérieur de grotles, de chammières, de hiosques, de fabrípues, de mille agrémens divers.

Le talent du jardinier-architecte embrasse toutes les localités, et ces localités différant dan leur disposition, les plans varient comme les sites de la Nature : tirer parti d'un monticule, utiliser un rocher, en créer méme d'artiliciels; rechercher les points où la vue doit se porter avec plaisir; masruer avec intention des objets qu'on ne verrait ‘fu'arec répugnance ou sans intérêt : telles sont les lois prescrites par le goût et la raison. H. Grabricl Thoinh les connait et s'y conforme; it doit ètre consulté comme un excellent guide par tous ceux qui s'occupent des Jarlins d'agrément.

Linsi, quoiquà des titres différens, sans avoir d’autre point de rapprochement que d'ètre les ceuvres de deux frères distingnés l'un et l'autre, chacun en sa partie, ces deux Ourages métitent d'être en même temps recommintés à tous cenx qui, dans la disposition et la culure des jardins, visent à rćuniv l'agréable à l'utile.

Ils nous offrent, sous ce commun rapport, l'occasion d'accoler ici le double hommage que nous nous plaisons à reutre anx talens do deux fróres iuséparables dans l'expression de l'estione publique, comme ils le furent dans kur toucliante anticic.

La nombreuse collection do Plans, publice dans la première et la deuxièmédition, a élé favorablement jugée.

La troisieme édition que nous publions, outre qu'elle contient un plus grand nowbre de Plans, présente ne anéliolation renurefuable.

Les Planches, entièrement noires, dans les ćditions précédentes, avaient l'inconvéuient de ne pas permettrè à l'ceil de discemer, au premier aspect, les parties sablées d'avec celles occupées par les can ; pour éviter toute confusion it cet égard, el rentre lus divisions toul-à-fait distincles, elles ont été mises cn couleur de nuances analogues, qui donneat à ces dessins tonte: la clarté désirable, et épargnent les frais l'une enluminure complète, beanconp plus dispendieuse.

L. B.

(1) Cours dle culture et de naturalisation des vigetaux, par André Thouin, mumhre de l'Tustitut, professeur de cuiture au Muséum (l'Histoire natırelle, etc., pulslic par Oscar Leclerc, son ncven, et son aide au Jardin du Roi. Trois volumes in- ${ }^{\circ}$, imprinnés avec des

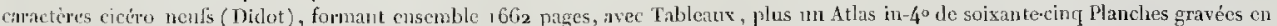
taille-donce, représentant tous les Outils, Instrumens, Lstensiles, Machines, et Fil rirques cliverses, de grande ou de petite culture, dout les Moléles compusent la cullection furmée du Jardin du Roi, et les exfmples de travanis dopérations de Culture, dessinés pour liz phupart d'aruris nature, dans l'École pratiq̨ue de cet Établissement, ctc. A Paris, clez Madame Huzard (née Vallat la

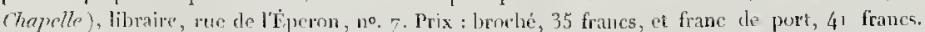




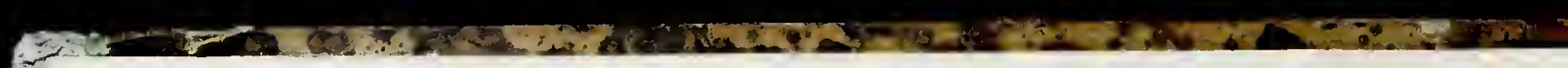




\section{PREFACE DE L'AUTEUR.}

Potr mettre le Public à portée de connatire la uature ct l'étenrlue de l'ourrage que nous publions, nous allons exposer rapidement quelques vues générales, et les principaux caractéres propres et particuliers aux différens Jardins.

On peut diviser les Jardins en quatre sections principales, en raison de leurs divers usages

La $\mathbf{I}^{\text {1e }}$. comprend les Jardins économiques ou légumiers, à eommencer par ce quon appelle marais, près des grandes villes, jusqu'à ceux que l'on nomme potagers, qui accompagnent presque tous les!grands Jardins de plaisance.

La $2^{\circ}$, se compose des Jardins fruitiers ou vergers, daus lesquels les arbres sont ou abandonnés à leur croissance naturclle, ou soumis à l'art de la trille

La 3", renferme les Jardins de botanique destines it lis culture des séries plus on moins nombreuses de plantes propres à l'étude de cette seience, on muployées daus la pharmacie et la médecine.

Dans la 4 , enfin, se trouvent les Jardins d'agrćment ou dc plaisance, dont le nombre rarie à l'infini, suivaut les localités, la nature du sol, la situation et lé climat.

Ces Jardins doivent être divisés eux-mêmes en trois séries principales, saroir

$\mathrm{I}^{\circ}$. Les Jardins symétriques, á la composition desquels procedent la régle et le compas, et que l'on exécute au moyen de la toise, des jalons et du cordeau;

$2^{\circ}$. Les Jardins clubois, anglais ou de genre irrignlier, qui n'ont ponr principes que le caprice ou la fautaisic de leuts constructeurs et les facultés de leurs propriétaires;

30. Les Jardins des paysages, paysagistes, paysagers, on de la nature (noms que l'on dome dans les divers ouvrages qui en traitent spécialement).

Les Jurdins symétriques u'admeticnt dans lene composition que des formes régulières, et des surfaces plus ou moins planes daus leurs parties ou mème dans leur cisemble. 'J'cls sont les Jardins tu Palais-Royal, du Luxembourg, des Tuileries, Ie Versailles. L'architccte le Nùtre, au conmencment du siècle dernier, a fourni les plus Jraux modẻles en ce genre.

Les Jardins de la deuximme série offrent, dans un espace tris-rétréci, toute sorte de formes fantastiques, en méme temps que les diverses productions des arls et les fabriques de toute espéce, amoncelées sans nécessité comme sans rapports entre elles. Tels étiient les Jatdius de Honcean et de Chavilles, tels sont encore une grande partie de ceus cqui ont étei exécutés it Paris, flans les temps modernes.

Enfin, le caractére de lát troisième ct dernière série des Jardins l'ngrénent est d'imiter les plus belles scènes de la Nature, en faisant disparaître l'art qui a servi ì les ćtablir. Ceux dEmnenonville, de Guiscard, de Méróville, de Trianon, de Jambeville, de Moulin-Joli, construits par Watelet, de l'Académie française, Girardin, Morel, Bulanger, MI. Lecourbe, Blaikié, etc., ofrnient et présentent cucore de beaus exemples te cette série de Jardins, chantés aroc tant de grâces par Defille, daus son poüme des farclizs.

Ceux-ci ne doivent pas être confondns avec les Jardins qu'on nomme communemeut chinois on anglais, prisque c'est la Nature qui a foumi leur modéle, el que les principes dianè lesquels ils sont établis ont été posés en France, dés le commencement du siede dernice, par Dufreny. Cet architecte a donné un bean modelt: de cette construction sur nn terrain qui lui apparienait, dans le faubourg Saint-Antoine, à l'aris. I,es plans qu’il présenta à Louis VIV des vastes Javlins de Versailless, de Mendon et de Siml-Germain-en-Laye, doni quelques-uns ont été gravés, suffisent pour lui assurer l'antériorité sur ses coucurrens en ce genre.

Cette série des Jardins paysagistes ou de la Nature ofle cinq sections diffërentes, qui comprennent les Jardius champétres, syluestres, pastoratex, ronantiques, et les parcs ou carrières. Ces noms lenr ont été donnés en raison des caractéres qui les distinguent dins leur ensemble, et dont nons tracerons ici une légére esquisse.

Un sol plane ou peu tourmenté, des prairies, des terres labourables, des cultures économirpes, des vergers agrestes, des bourguets de bois, des masses fleuries, une culture soignce, des eaux vives, des fabric] nes agricoles et des rues uénagées sur tout le pays environnant, avec lequel ils paraissent se confondre, constituent les Jardins du style champeitre.

On donne le nom de sylvestres á ceux dont le sul, àpre et toumenté, présente des rochers, des chutes d'eau, des forêts d'arbres estivanx et résineux, đles clairières tapissées de gazon et émaillćes de fleur's des diverses saisons; des faloriques approprices au site, des chaumiẻres agrestes de bucherons ot de charbonniers, augmentent les caractéres distinctifs des Jardins de celle suction.

Ceux de la troisicme ou do style pastoral exigen des terrains unis ou nu pen concaves, traversés par des eaux vives, formant des ruisseanx, de petites rivières, des lacs bordés de pelouses, de prairies, d'oseraies, de saules, de bouquets d'arbres aquatiques variés par leur port et leur hantenr, des ponts, des moulins, des bestiaux de plnsieurs espéces, des cabanes rustiques propres aux animanx, qui animent la scène, et aux honmes quì les gouvernent. 
(B11 alpelle Jaudins romentiques ceux dont le sol, très-varié dans son plan, ainsi que dans ses élévations et ses contou's, présente des pieces de gazon, des tapis de fleur's, des nusses d'arbustes, des bouquets d'arbres d'agrénent de toutes fes saisons, des bois dans leurs diférens àges, des futaies, des canx dans les divers états dans lesquels on les rencontre dans la nature. Ces Jardins admettent, pour ornement, des vases, des statues, des colonnes, des grottes, des rnines, dis combeaux et des temples.

Enfiu, lat cinquíme et derniere sorte des dardins-paysages, nommé parc ou carriere, néecsite les phus grandes dimensions dans son ensemble. Un pare comprend sourent un pays cutier : celni de Versailles, par exemple, renforme le Jardin du palais, les chateanx et les Jardins des deux Trianons, les fermes de satori, des hameaux, des villages et des bourgs. Les fardins de celle section admettent tout ce qui distingne les unatre précédentes, tous les genres de culture, tons les batimens, depuis les palais des sonverains jusqu'à la cabane du cliarbonnier et la luutte Iu berger. Ils comportent l'emploi des eatu sous toutes les formes daus les phlus grandes dimensions, toutes les usines ct les fabriques, tous les ehemins qui doivent servir à les pareonrir ou les traverser : ils admetteut lous les

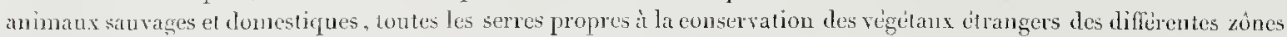
Te la tcre. Mais il fant que chaque sène qui se presente aus regards, cucadrece dins ses limites, n'ofie pas de contraste cloquant of encore moins de contrarlictions; il faut qu'elle soit lice aux autres par des transitions ménagées

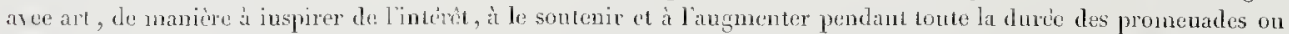
des eourses. Comme elles se font ordinairement id cheral ou en voiture, il est essentiel d'établir, dans les roisinages te l'habitation, des allées circonscrites dans de petits espaees qui puissent servir aus promeneurs ì pied, à toutes les leurus du jonr et dans tontus les saisons; de former des sentiers on chemins qui conduisent directement à ehaque site en partieulier, el enlin des routes qui fornent les linites de la proprieté, et, la traversant dans tous les seus,

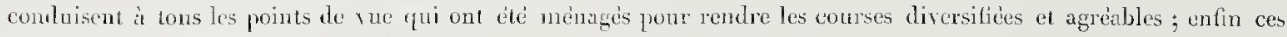
compositions doirent rassembler les sites les plus gracieux el les flus surprenans, et emprunter aux arts mécani-

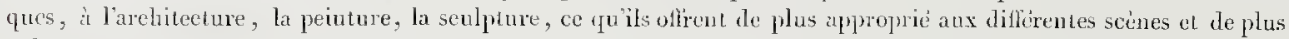
seillitisant,

L.eo Jardins de Guiseard, te Chanteloup, de Bay, de Chantilly, d'Ermenonville, en France; ceux de Stowe, de Persichd, de Haglay en Angleterre; en Allemagne, eeux de iymphenbong et de Munich, et entin, si l'on cru croit les relations, cenx des eurperen's de la Chine aus environs de Pekin, présentent, ou du moins présentaint, ear plesieurs d'entre cux n'existent plus, des modéles ylus ou moins perfeetionne's de ee genve de Jardins.

Il existe un grand nombre d'onvages pulolies en livance, en ingletere, en Nlemagne, etc., qui traitent avee demilue de la theoric de la composition des diflèrens genres de Jadins dont nous venons d'esquisser l'énnmération. Mais il en est peu, on mime point, ì notre connaissance, dans lesequels la pratique de cet art soit développic par des plans exacts, dont loutes les parties figurées soient dans leurs justes proportions et en xapport avec le caractere essentich de la constrnetion. Aucun deux ne présente une série de modeles de divers geures, diluries lesquels on puisse exécuter sur le terrain ceux qui pouraient ètre à la biensénce des propriétaires des diverses classes de fortune.

Notre but n'est point de nous oceuper de la throric de l'art de la composition des Jardins, qui, ayant été traitie dans beaueoup d'ourrages, comme il vient dêtre dit, deviendrait iei superflue; mais nous avons cru qu'un our rage qui présenterait wne sute de plans de toutes les sortes de dardins pourrait itre utile et agréable au public.

Tons nous proposons done de lui offir une série non interompue de tontes les constructions en ee genre, depuis celles qui se trourent en raport avec les faeultés du modeste labitant des campagnes, jusquä celles qui conviennent aux hommes les plus fortunes et aux Puissances de la terre. Nons traeerons le petil clos ferme d'uno haie qui entoure la channice dn laboureur, "t dont l'espace, divisi en earrés ou planches, est oceupé par des

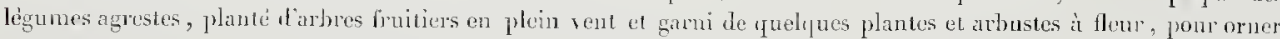
le corsel de la jenne villageoise lé jours de fète. Nont nonblierons point la tonnelle ou le bereean seus leçuel la

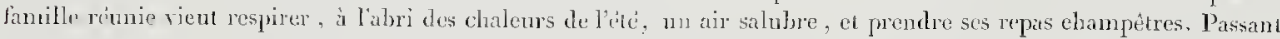
ensuite anv Jardins des citadius qui entoment lis maisons des villes, nons offirons des exemples om plutôt des mortéles de leur construction. Cens-ci dovent présenter un marcher facile pour l'exereice de la pronenade, nne verdure qui repose at rojonisse la vue, en mème temps que des fleurs qui lauttirent et la flattent, des odenrs qui captivent lodorat, des frnits qui prissent satisfaire le gout ; mais essentiellement nn air pur et Lalsamirque capable de neutraliser eelui que l'on respire daus les appartemens fermés et dans le eloaque de la pluprat des rues.

Nous oflirons des exemples du genre symétriqne, soit que les lardins qui le composent sojent destinés à la culture des légumes, des flenrs, des jennes arbros en prininières, on que leur usage soit de servir de délassement au public,

Les dirdins paysagistes des diferentes sulies seront traités ave tonte l'étendue que néeessitent leurs nonbreuses varictés et la diflérenee des climats du nidi, du milien, du norl de l'burope, oi l'on serait tenté de les exécuter, mais tonjonrs en olliant des modèles qui puissent étre cu rapport avee les fucultés pécuniaires des diverses classes de la secicité.

Les élénens de cet our rage sont le fruit d'observations multip,iées et d'une pratique de plus de einquante ans. Nous avons exécute la plupart de ees plans en divers lieux; les autres ont été faits sur des programmes qui nous sont parvenus des pays étrangers et propres à êtce exécutés dans les différentes parties de l'Europte. 


\section{INTRODUCTION.}

$A$ Avant de domncr les détails particuliers sur chaque Jardin paysagiste on d'agrément, on a pensé qu’il convenait de faire connaitre les Olsservations gènèrales ci-jointes:

$I^{n}$. Le Bâtiment principal doit av oir des Points de Vue agréab]cs sur lé Jardin, pour cngager à la promenade, et exciter la curiosite par des Monumens, qui auront eux-mèmes leurs Points de Vuc sur la Campagne ou sur quelques Fabriques;

$2^{\circ}$. Il faut planter sur le derant du Batiment, des Arbres verts et autres, dont la teinte du feuillage soit foncée en coulcur, pour faire repoussoir. Sur le sceond Plan, il faut des Arbres plus petits, et dont le feuillage soit plus clair;

Enfin, sur le troisième Plan, les Arbres doivent ćtre plus petits, et avoir une teinte argentine, afin cle rendre, sur le terrain, lefict qu'un peintre de paysages rend sur la toile.

$3^{\circ}$. On doit établir un Chemin qui tourne autour de la Proprićté, en observant de l'alonger le plus qu'il est possible, et lui donner un contonr agréable, pour qu'en le parcourant, soit ì pied, á cheval, ou en caicche, on u'éprouve aucune difficulté, ct pour ne pas suivre l'exemple dounć dans des Jardins, soi-disant Anghiuis, où l'ou voit des allées qui tortillent sans motifs, et qui ne mèncnt ì aticuu but.

4. On veillera anssi à ce que tous les Chemins qui se sépareront de celui cqui toume autour de la propriété, aicnt une destination, soit pour conduire ì difî́rentes Fabriques, Salle de repos et autres Points de Vuc, et à ce que claqque Fabrique fasse un Tableau. Il est nécessaire que tous ces Chemins soient bordís de diffërentes espececs d'Arbres, soit en massifs, ou isolés.

Planter des masses d'Arbres de mème nature, et si on les mẻlange, rassembler les teintes í-peu-prés semblables, afin d'éviter le désagrément de voir des couleurs fortes réunies à des conlenrs faibles, ce rui produit un contraste désagréable. Il faut avoir soin ègalement de grouper de maniére à former toujours des avant-sénes prour chaque Tableau qu'on aura à faire, et les varier. Les Arbres les plus agréalules doivent être plantés isolẻnent dans la prairie gè̀ et lì par groupes de $3,5,7,9$, etc.

Dans le choix d'une Propriété, il serait agréable d'avoir le Bàtiment principal sur une hautcur, afin que du Bátiment on puisse apercevoir une partie des Fabriques ćtablies à divers endroits, et former le chemin aux angles dudit Bàtiment, afin 'fu'il se trouve entouré de pelouses de tous côtės.

Il est à observer que les Plans des Fabriques sont à l'échelle du Plan; nais on a èté obligé de faire quelquefois les élévations du double, triple, etc. du Plan, nais toujours daus les uuèmes proportions, pour rendre les détails plus scusibles. 


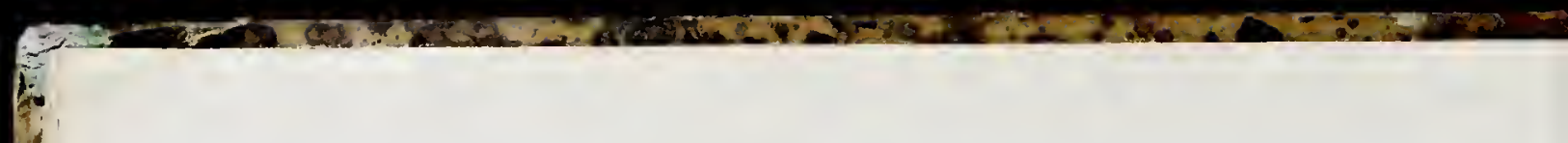




\section{PLANS RAISONNES}

$\mathrm{DF}$

\section{TOUTES LES ESPECES DE JARDINS.}

\section{$N^{\circ}$ I. JARDIN DE GROS LÉGUMES.}

C

Tardin contient environ six arpens, et pourrait ètre clos de haies. On y cultiverait toutes les espèces de Légumes, nécessaires à l'approvisionnement des halles, comme clioux, pommes de terre, betteraves, carottes, laricots, navets, et autres dı nicme genre, selon la nature des climats et les productions des différens pays.

A. Bàtiment du Cultivateur.

B. Bàtiment destinć à serrer les légumes.

C. Basse-cour avec toit à porcs, vacherie, écurie, trou a fumier ct colombicr.

D. Puits autour desquels on planterait des arbres fruitiers pour garantir du soleil les ouvriers qui $y$ tireraient de l'eau.

E. Carrés destinés aux semis des légumes potagers; ces carrés seraient entourés de plates-bandes, garnies d'arbustes à fleurs et de plantes d'agrémens.

F. Massif ou on planterait les différentes espèces de Légumes potagers. Près du Bàtiment, il y aurait un berceau ou tonnclle; autour de ce berceau, on planterait des arbres d’agrément atx pieds desquels on mettrait des plantes grimpantes ou des vignes.

G. Massifs de groseillers, au centre desquels on planterait un noyer ou autre arbre à fruits; tous les carrés, on massifs de ce Jardin, pourraient être entourés de vigues.

\section{$\mathrm{N}^{\circ}$ 2. JARDIN POTAGER MARAICHER.}

Ce Jardin contient cuviron quatre arpens. Le terrain est supposé entre deux rivières qui se joignent à l'extrénité dudit Jardin. On a pratiqué plnsieurs escaliers pour pouvoír puiser à la riviẻre de l'eau pour les arrosemens du Jardin.

A. Bâtiment du Jardinier. Son logement serait élevé de quatre pieds au-dessus du niveau du terrain, et quatre pieds pour descendre à la cave; ce qui donnerait huit pieds pour le dessous du Bảtiment : il serait destiné à serrer les Légumes et autres objets.

B. Couches à melons sous chàssis.

C. Couches à melons sous cloches.

D. Couches à champignons.

E. Cour à fumier et Hangar pour serrer les cloches, paillassons, et les instrumens aratoires.

F. Côtièze destinée aux primcurs.

G. Clıque planche serait destinée à mettre différens Légumes.

II. Puits. 


\section{$\mathrm{N}^{\circ}$ 3. JARDIN POTAGER PRIVÉ.}

Ce Jarlin eontient trois arpens un tiers, et pourrait être établi dans le miliea d'un pare ou d'un jardin pittoresque, et serait entouré de murs, le long descucls on planterait des pêchers, abricotiers, vignes, ete.

A. Bâtiment de Cultivateur. De claque côté il y a une Serre; en avant des deux Serres, il y aurait deux cllẩssis destinés aux semis.

B. Ilangard pour serrer les ustensiles du jardinage et la voiture.

C. Cour a fumier.

D. Puits auquel on a adapté une manivelle pour tirer l'eau, la dirigger dans le réservoir et ensuite dans les bassins établis dans le Jardin, au nombre de quatre.

L. Réservoir.

F. Figueric ou Melonnière.

G. Quatorze carrés destinés à la culture de toutes les espèces de Légumes, lesquels seraicnt cntourés, ainsi que les murailles, d'arbres fruitiers en espaliers.

\section{$N^{\circ}$ 4. JARDIN POTAGER DE PRIMEUR.}

Ce Jardin contient deux arpens et demi. Il serait spécialement destiné aux Serres, chàssis et couches.

A. Grande Serre pour chauffer les figuicrs, cerisicrs et les Légumes de primeur, arec un poële sur le desant de la Serre, posé sur l’épaisscur du mur d’appui, et sur le tuyau, qui serait caré, on mettrait des poteries, pour fraisiers, ou antres Légumes.

13. Petite Bache ou chatssis hollandais en arant de la grande Serre; dans laquelle on semerait des haricots; des pois, et on planterait des fraisicrs afin d’y récolter de bone leure. Un fourneau en terrecuite serait placé sur l'épaisseur du mur qui cst en avant.

C. Serre à ananas.

D). Baclie à ananas.

E. Serre que l'on adapterait le long des murs pour chanffer les arbres en espalier, comme vignes, abricotiers, pechers et antres. Sur le derant de ladite Serre, on pourrait semer des prois, des haricots et autres. Pour ne pas fatiguer les arbres, on ne les chauffe que tous les trois ans. Si on a un espalier de soixante toises, on cul chauffe tous les ans ringt toises.

Pour établir cette Serre, on place des dés en pierre à quatre pieds de distaure les uns des autres, pour recevoir les moutans en bois sur lesquels on pose les chàsis. Il faut que les dés ayent dis-huit pouces d'écarissage, afin de pouvoir entailler, dans lesdits dés, le diamétre des tuyaux en terre cuite destinés pour la conduite de la chaleur, comme il est marqué sur le plan E.

F. Châssis dont les couches scraient inclinées pour les melons.

G. Chàssis, dont les couches seraicnt à plat, pour les melons, asperoges, laitues, etc.

II. Couches inclinées pour melons, laitues, sur lesquelles on mettrait des cloches.

I. Couche ì plat eomme ci-dessus.

K. Couchies sourdes.

l. Couches sourdes inclinées pour les patates, etc.

MI. Couclies sourdes cr buttes, pour melons, patates ou autres.

N. Couclıes sourdes pour succéder aux clıàssis.

O. Couches sourdes à plat pour succéder aux couches à cloches.

P. Couches en meale à clampignons. 


\section{$\mathrm{N}^{\circ}$ 5. ORANGERIE.}

Ce Jardin contient environ six arpens.

A. Serre d'Orangerie.

B. Grande plate-bande en gazon sur laquelle on mettrait les plus grauds Orangers.

C. Deuxieme plate-bande sur laqueile on mettrait les moyens Orangers.

D. Troisieme plate-bande sur laquelle on mettrait les plus petits : ce qui ferait amplhithéâtre. On établirait les plate-bandes plus larges que la tête des Orangers, afiu que les fleurs ne tombasscnt pas sur le sable.

E. Pelonse autour de laquelle il $\mathrm{y}$ aurait des plate-bandes de fleurs sur lesquelles, et cn face des pelits Orangers, on planterait des rosiers grefẻs sur églantiers, ce qui terminerail l'anplithéâtre. Lorsque les Orangers seraient dans l'Orangerie, on mettrait a leur place des arbres verts, ce qui formerait un amphithéàtre d'un antre genre.

F. Allćc faisant le tour de l'Orangerie d'été.

G. Grand bassin.

H. Salon qui aurait vue sur le Jardin de deux côtès. Le côté de la glace serait placé du còté de l'Orangerie de manic̀e à produire l'effet suivant:

Il y aurait denx glaces, l'une à tain el l'autre sans tain; toutes denx seraient mobiles et de manièce à disparaître à volonté. Lorsqu'on recevrait des personues dans le salon, la glace à tain serait en évidence; lorsque la compagnie aurait été distraite par un deplacement, on la ferait revenir dans le salon; alors on aurail fait disparaître la glace à tain pour y substituer celle sans tain. La surprise serait grande de voir au travers de la glace une Orangerie illuninée. A l'extrémité, parallèle à ce salon, serait un Pavillon pour la musiquc. Les portes intéricures du salon, étant garnies de glaces, répéteraient l'Orangeric.

\section{$\mathrm{N}^{\circ}$ 6. JARDIN FRUITIER NON SUJET A LA TAILLE:}

Ce Jardin, de huit arpens, serait entouré de haics ou d'un treillage agreste; les plantations se feraient d'une manière irrégulière ou en ligne, et cn laissant assez de distance pour que le fourrage pousse facilement, et que les arbres ne se gèncut pas entre cux.

A. Bàtiment servant à serrer les ustensiles aratoires, et logement du jardinier.

B. Fruiticr.

C. Grange.

D. Basse-cour dans laquelle on planterait des noyers, mûriers ou autres arbres.

E. Vacheric el écurie.

F. Polager.

G. Porte rustique.

H. Salle de repos.

I. Petit Bàtiment pour loger un garçon jardinier chargé de veiller à la conservation des fruits.

\section{$\mathrm{N}^{\circ}$ 7. JARDIN FRUITIER EN QUINCONCE.}

Celui-ci peut étre établi dans un Jardin pittoresque; sa grandeur serait de deux arpens et demi environ.

A. Bàtiment selvant de logement au jardinier. Ce Bàtiment pourrait faire point de vue au Château.

B. Cour pour les fumiers. 


\section{( I 4 ; )}

C. Ilangar pour serrer les écliclles destinces à la taille des arbres.

D. Bassin.

E. Massifs entonrant le verger.

\section{N 8 . JARDIN FRUITIER}

\section{SLJET A LA TAILLE EN QUENOUILLLS.}

Ce Jardin contient trois arpens et denii. Il pourrait ètre établi dans un jarlin pittoresque, en y ménagcant des clairières pour domure le lair aux nourelles plantations.

A. biatiment du jardinier.

B. Pelouse.

C. Massifs d'arbres en quenouille.

D. Clairières.

$\mathrm{N}^{\circ}$. Taille d'un an.

Yo 2. Taille de cleox ans.

No 3. Taille de trois ans.

\section{$N^{\circ}$ g. JARDIN FRUITIER}

\section{SUJET $\Lambda$ LA TAILLE EN VASES OU BUISSONS.}

Ce jardin contient quatre arpens et demi. Il ponrrait ètre établi dans un jardin pittoresque : les arbres seraient plantés de manière à être taillés commolément; il faudrait ménager des clairičres pour faciliter la végétation.

A. Bàtinient du jardinier, servant de point de vue. I un des angles du batiment, il y a une tour gothique, de laquelle on peut découvrir toute la campagne.

B. Massifs darbres à fruits.

C. Pelouse.

1). Clairicres.

$\mathrm{N}^{\circ}$ r. Taille l'un an.

$\Gamma^{\circ}$ 2. Taille de deux ans.

N 3 . Taille de trois ans.

\section{$\mathrm{N}^{\circ}$ г. JARDIN FRUITIER}

\section{SUJET A LA TAILLE EN ESPALIERS.}

Ce jardin contient quatre arpens deux tiers. Il pourrait ètre établi dans un jardiu pittoresque. Il est uecessaire qu’il soit entouré de murs afin de pouvoir établir les espaliers. Il serait pratiqué quatre issues pour aller dans le jardin pittoresque autour des murs. Il y aurait une plate-bande ou on planterait des 


\section{$(15)$}

arbres fruitiers, comme pếchers, abricotiers, poiriers, cerisiers, etc., et entre les arbres, de la vigne pour former un cordon au-dessus des espaliers.

A. Bâtiment pouvant scrvir de point de vue au Clâteau, et de logement au jardinier.

B. Porte rustique.

C. Bassin, au centre durquel il y aurait un jet d'eau C. ou une figure D. représcıtant Pommone, déesse des fruits.

E. Arbres fruitiers, taillés en érentail, plicés sur des plate-bantles.

F. Allée fiasant le tour du verger.

G. Espalicers.

N" r. Taille l'un an.

No 2. Taille de deux ans.

$N^{\circ} 3$. Taille de trois ans.

\section{$\mathrm{N}^{\circ}$ II. JARDIN PIIARMACEUTIQUE.}

Ce jardin contient deux arpens un quart.

A. Bàtiment du cultivateur.

B. Serres destinées à mettre les plantes qui doivent être portées à la halle.

C. Deux autres petits bàtimens destinćs à éplucher les plantes et fleurs, pour ètre livrées aux herboristes.

D. Monticules, plantées d'arbres isolés, sous lesquels on placerait les plantes qui demandent de l'ombre et de la sécheresse.

E. Plate-bandes de différentes grandeurs; les grandes, pour les plantes dont le débit est considérable, leș moyemnes et les plus petites, par gradations, pour les plitutes dont l'usage est moins frérquent.

F. Bassin pour les Plantes arpuatirucs.

G. Isle plantée de manière que claque espèce ait la quantité d'eau suffisante pour leur régétation.

iI. Allée plantée de tilleuls dont la fleur est utile, et ils procureraient de l'ombre et une promenade agréable.

I. Petit bassiu pour l'arrosement.

Plantes que l'on peut cultiver avec avantage dans le Jardin Pharmaceutique, on obserpant le site convenable aux espèees.

Absinthe grande et petite.

Aconit napel.

Ache.

Angélique.

Armoise.

Aunćc.

Bardane.

Belladone.

Bourracle.

Camomille.

Chamedrys.

Chardon bénit.

Chicorée.
Ciguë.

Guimauve.

Hysope.

Mélisse.

Menthe.

OEillets rouges.

Raifort.

Ruc.

Roses de Provins.

Sauge.

Scordium.

Violettes. 


\section{(16)}

\section{$\mathrm{N}^{\circ}$ I2. JARDIN PIIARMACEUTIQUE D'ÉTUDE.}

Ce Jardin contient Ileux arpens et demi.

A. Båtiment destinć pour la démonstration des plintes et drogues.

B. Salle fon la préparation des sroģues.

C. Escalicr pour monter au secont, oi serait le logement des Professeurs, et plus Iaut le logement du jardinier et des persomes occupees it la culture.

D. Jicuries, remise, poteric et lieu oil l'on prẻpare les terres.

E. Cour d'entrće.

F. École de botanique.

G. Bassin servant a l'arrosement des plantes.

H. Promenales, avec bancs le long du inur, pour les éléres.

I. Sere dOrangerie.

F. Serre tempéréc.

I. Bache.

M. Chaissis.

N. Plantes destinées pour ètre distribuées aux éleves. Ce terrain contient huit cent quarante toises.

O. Bassin pour les plantes aquatiques.

P. Hangar ponr serrer les ustensiles aratores.

Q. Vestibule on on mettrait les fourneaux de la serre posés sur le petit mur d'appui qui porte les vitraux.

R. Passage pour aller d'un jardin à l'autre.

Dans une Ĺcole destinéc á l'instruction des èlèves, le Professeur ćtablit la méthode, la classification ou le systeme qu'il juge le plus convenable pour faciliter lètude des végétaux. Les Écoles les plus célères ont adopté les fimilles naturelles de Jussicu, d’autres le systeme de Limeus; ceux-ci la métlode de Tournefort, avec quelques modifications.

Il est loin de ma pensée de vouloir tracer aux Professenrs lia classffication quils doivent suivre; mais, puisque l'Ecole du Jardin du Roi a adopté la méhode de Jussien; que le Professeur de la Faculté de Médecine de Paris suit le systime de Linneus, avec quelques changemens, je vais rapporter la classification enseignér à l'Ecole Spéciale de Plarmacie de Paris.

Cette méthode, adoptée par Tournefort, et modifiéc par M. Gruiart, est eelle qui convenait mieux, tanl par rapport à la célébrité de son auteur, qu'à cause de son utilité pour le jeune pharmacien, qui se livre pour la premicre fois a l'etude de la Botanique.

MÉTIODE CALQLÉE SUR CELLE DE TOURNEFORT.

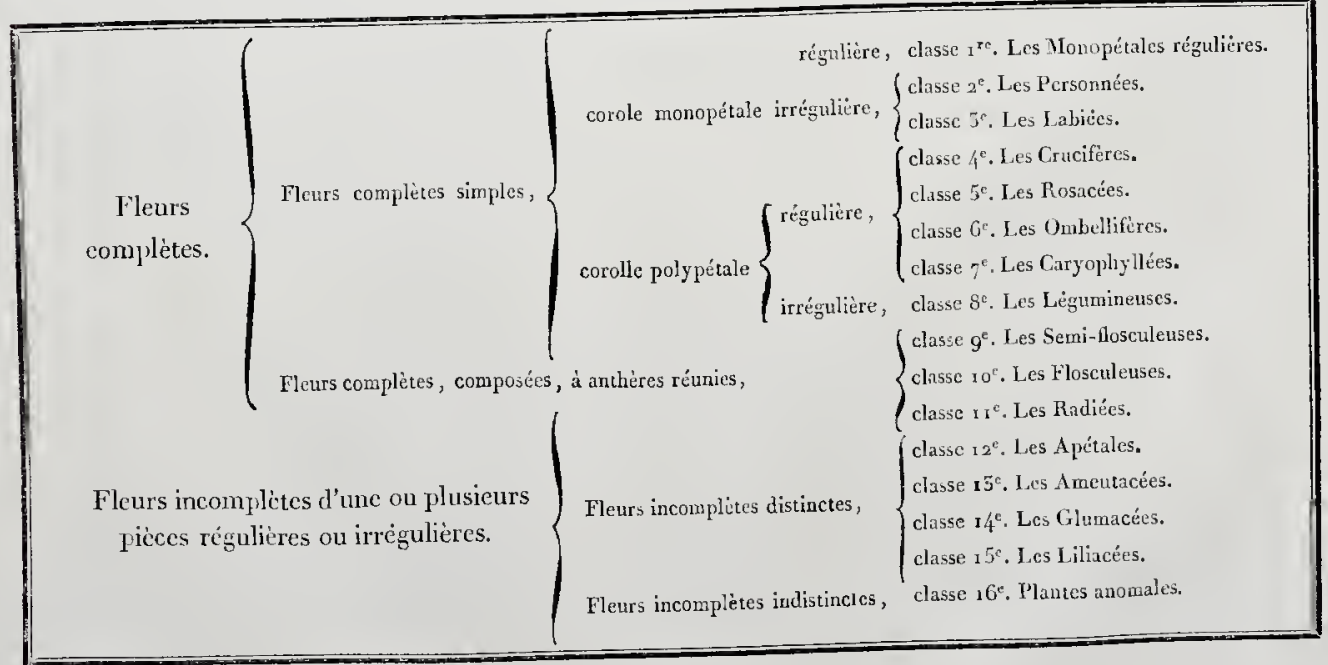




\section{No 13. PROJET D'AGRANDISSEMENT}

\section{DU JARDIN DES PLANTES DE PARIS.}

Ce jardin contiendrait cent quarante arpens, et scrait destiné á la culture de tontes les espéces de végétaur.

A. Cabinet d'Histoire Naturelle actuel.

AA. Cabinet où seraient classés les trois régnes de la nature.

B. Ecole de Botanique, fermée par des caux vives, des eaux stagnantes, des eaux eourantes et des viviers, dans lesquels on mettrait tontes sortes de poissons pour les démonstrations.

C. Bassin propre à la eulture des plantes aquatiques.

D. Plantations d'arbres variés, les plus petits sur le devant, les moyens sur la seconde ligne, et les plus grands strr la dernière ligne, de maniére i former un amphithétitre sans être obligé de les tailler; ce qui donnerait un aperç de la nature de charue axbre, pour les planter dans les massifs des jardins piltorestues, et propres à offrir un coup-d'oil agréable.

E. Pépinière dans plusieurs carrés. Il y anrait dés écoles de démonstration pour les greffes, la taille, les boutures et marcottes.

F. Potager, avec des murs de refend pour les espaliers et la démonstration des différentes tailles.

G. Fleniste.

II. Serre chaude, scrre tempéréc, scrre á annnas, bache pour la naturalisation et autres.

I. Dépót de fumiers, terres, poteries, ustensiles, et langar derrière les serres.

K. Bibliotlèque.

I. Grange pour serrer les produits de la culture.

M. Amphithéatre pour les démonstrations de botanique, de chimic et autres.

N. Logement des professeur's et corps-de-garde.

O. Grand labyrinthe.

P. Pelit labyrinthe.

Q. Serre Baudir.

R. Serre des arbrisseanx.

S. Serre des plantes grasses.

T. Serre chande.

U. Orangeric.

$\boldsymbol{V}$. Rocher d'où l'eau sortirait pour retomber dans un las ó il y aurait plusieurs îles. Le eheanin $\mathrm{X}$ passerait sous le rocher, et. les eaux tomberaient á travers les roclies, devant les personnes qui iraient au Cabinet. $\Lambda$ l'entrée du soutcrain, il y aurait des escaliers pratiqués dans les roches, conduisant au labyruthe. Sur le rocher on placerait tontes les especes de plantes srasses, pendant l'été.

Y. Moulin á eau, propre à des divers usages.

Z. Parc pour les mimaux paisibles.

\&. Moulin à vent.

(a) Vignoble pour toutes les espéces de rignes qui croissent en Europe et autres.

(b) Ferme contenant vacherie, bergevic, grange, colombier, toit à porcs, elc.

(c) Toliere entourćc d'arbres.

(d) Ménagcric des animaux féroces, avec leurs parcs.

(c) Autre ménagerie d'animanx paisibles.

(c) Terrain destiné a la culture des plantes usuelles distribuées aux paurres malades; pour la teinture, la filature, et une plate-bande dont les plantes sont données aux ćléves qui font des herbiers. Les platehandes qui bordent les arbres seraicnt destinées aux arbustes, arbrisseaux, qui se lieraient avec les petits et grauds arbies. 
(s) Portes d'entréc par la rue du Jardin du Roi.

(h) Pont d'Austerlitz.

(i) Pont de la Tournelle.

(k) Grande culture pour les céréales, avec des arbres plantés gà et là, par groupe, et pour en aroir les graines.

\section{$N^{\circ}$ I. . JARDIN POUR LA NATURALISATION.}

Ce Jardin contien environ quatre arpens.

A. Bâtiment dı cultivateur.

B. Orangerie faisant parallèle.

C. Serre chaude ponr planter les arbres en pleine-terre.

D. Serre pour les arbres de lia Nourclle-Hollande et autres.

E. Baches pour la multiplication.

F. Baches au couchant, pour le mème objet.

G. Plate-bandes pour la multiplication.

H. Plate-bandes pour les semis.

I. Triangle destiné aux semis.

Ii. Grand carré pour les arbres un peu forts.

1. Carré pour repiquer les semis.

N. Bassin.

O. Fleuriste.

P. Plate-bandes pont flacer les arlores et arbustes en sortant des serres.

Q. Carré pour repiquer les semis de deux ou trois ans.

S. Escalier descendant de la terrasse au fleuriste par-dessus la bache du nord.

T. Bache du nord.

U. Testibule pour allumer les fonrneaux des serres.

V. Elération de la serre cliaude $\mathbf{D}$.

X. IIangar nour serrer les ustensiles du jarlinage.

Y. Escalier pour monter sur la galcrie de la serre D, pour la couvir aver des paillassons. $\Lambda$ l'extrémité de ladite salerie, du cóté de la serte $\mathbf{C}$, il y a un esealier touruant daus deux colliers de fer pour monter à la galeric de la serre $\mathbf{C}$ et au srenier destinć à serrer les paillassons, les grames, etc.

Le jardinier pourrait parcourir ces serres sans sortir dehor's.

\section{$N^{\circ}$ 15. JARDIN SYMÉTRIQUE DE VIILE.}

Ce Jarlin conticnt un demi-arperit.

A. Bìiment.

B. Allce couverte, faisant le tour du jardiu.

C. Allée de mème niveau allant au hosquet: clle serait séparée de la précédente par une haie en troënes ou en rosiers.

D. Allée du bosquet avee salle, bane de repos et diverses figures; ce bosquet est entonré d'arbustes à fleurs et darbres a tiges sur le dericre.

E. Allée creusée de dix-huit pouces, dont les terres seraient soutcnues par des talus plaqués en gazon.

F. Pelouse aux deux extrémités de laruelle il y aurait un vase et une ligure.

G. Sulle élevée de quatre pieds au-dessus de la pelouse $F$.

II. Autre salle, avec une figure, faisant fond au petit bosquet. Cette salle serait élerée de quinze pouces â-dessus, ce qui ferait amphilhéâtre.

I. Figures formant point de vue du Bảtiment.

K. Salle de repos du uéme niveau que la salle D. 


\section{No ${ }^{\circ 6 . ~ J A R D I N ~ P U B L I C . ~}$}

\section{CIIAMS ELISEES.}

Cette promenade conticnt environ cont viugt arpens.
A. Jardin des Tuilcries.
B. Place de Louis XV. Il y aurait à étublir deux ponts sur les fossés, l'un donnerait sur le quai des Tuileries el l'autre donncrait du còté de la rue de Rivoli.

D. Garde-Mcuble de la Couroume.

E. Pont de Jouis XY.

F. Clambre des Députés des Départemeus.

G. Palais Bourbon.

II. Quinconces des Invalides.

I. Cours la Reine.

K. Rotondes destinées pour les bals les jours de fêtes publiques, et lExposition des produits de l'industric nationale.

L. Grand carré des Jeux, aux angles duquel on établirait des pavillons destinés aux Restanrateurs; et sur la pelouse il y aurait des Jeux, les jours de fútes, tels que les exercies d'Ĺfuitation de Franconi, Dauscs de corde, Miats de Cocagnte et autres.

M. Carrés aux extrénités desquels on établinait des Cafés.

N. Ces deux carrés seraient disposćs de la mème manière, arec Traileurs ou Cafés.

Il serait élabli denx Avenues : l'une O traverserait le grand caré des Jeux, dont le commencement scrait à la place Louis XV; clle serait terminée par une Avenue quon pourrait établir à la lettre P. Cette Avenue conduirait du Cours la Reine an faubourg Saint-IIonoré ; ct l'Arenue Q commencerait ì la place de Louis XV, en suivant lalignement du Garde-Menble, jusquà la lettre P. Les jonrs de Fétes, l'on ponrrait illuminer ces trois grandes Avenues; celle du bord de l'ean, celle du milieu, l'Arente de Neuilly et relle du côté du Garde-Nenhle.

R. Jardins particulicrs.

S. Avenuc de Mirigny.

T. Arcnue d'Antiu.

U. Arenue des Veures.

\section{$\mathrm{N}^{0} 1 \%$ JARDIN DE PALAIS.}

\section{E S T U I L E R I ES.}

Ce Jardia, de liuvention de $\mathbf{M}^{* * *}$, fut commencé sous HenriIY, et séparé du Palais par une rue. Le Jardin dit Tuileries était mal distribué, dépourvu de tout agrément, et beaucoup noins ćtcndu qu'il ne l'est aujourd'lui. Les deux projets d'achever le Palais et d'enlyellir le Jardin furent conçus en mène-temps. Le innr fut abattu : on démolit les dircrs édifices qui s'y tronvaient, et sur le terrain rui contenait soixantc-sept arpcus, Lenôtre exécuta ce inagnifiqne Jardin, qui est regardé comme un des plus beaux de l’Europe. (Voye Saint-Yictor.)

A. Les trois pièces de broderies entourées de plate-bandes de fleurs.

B. Les trois bassins. 
C. Parterres de gazon.

D. Petit bois plantc.

E. Bosquets.

F. Boulingrin ou Bassin de gazon.

II. Salle de lia Comédie.

I. Frises on Paneaux de gazon.

T. Grand bassin octogone.

M. Escaliers.

N. Petil escalier.

O. Escalicr de la terrasse.

P. Terrasse la long de licau.

Q. Terrasse le long du palais.

R. Plan du palais des Tuileries.

S. Fossés de la rille.

U. Terrasse faisant face át la place louis XV.

V. Glacis pour monter sur la terrasse.

\section{$\mathrm{N}^{\circ}$ 18. JARDIN ROMANTIQUE CHINOIS.}

Ce jardin contient quatre-vingt-qnatorze arpens environ.

A. Porte d'entrie arec quatre parilions destinćs au portier.

B. Pont clinois.

C. Deus corps de bittimens serrant de restibule. Escalier et Galeric.

D. Salon du propriétaire.

E. Pont et Galerie courerte, conduisant au logement de divers amis.

F, Logement du proprićtaire ayant vue sur la mer. Un lac spacienx, entouré de montngnes cscarpées et dun chemin de ronde ì mi-côte, qui fail décourrir une partie de l'ensemble dn jardin, commode pour la promenakle.

G. He de Flore. On a plinté sur les bords des chemins, des arbres et arbnstes ì flenrs, et des fleurs odoriférantes sur de petites monticules très-donces, pour que les fletrs forment un amphithéitre, et "fue les parties en gazon soient un peu concares; ce qui donne de la grâce aux prairies, et fait que les clueninus ne sont pas monotones. On a $\mathrm{eu}$ soin, à ect effet, de les élever. Les massil's qui bordent les clicinuins de l'autre rivage, sont plantés d'arbustes a fleurs odoriférantes. On a placé des bincs clrampictres, l'oul, à travers les feuillages, on appercoit li rivière. Dans les partics un peu entourées, il y a d'antres massifs plantés de saules pleureurs, afin d'avoir la facilité de sapprocher á fleur d'eau pour pouroir y pè̀her. On a eu soin de placer sur les borls de lîle, des peupliers suisses et quelques saules pleareurs. A lextrêmité de l'ile se trouve un Tenrple chinois en prorceline, aperçu dı salon du propriétaire, et ayant vue sur denx rivières, l'une souterraine et lautre a lair, avec un front. Le pont de droite conduit i travers les roches, les pins, les sapins, les ronees, et rient gagner le eliemin I, qui passe sous un roclıer, ou l'on voil tomber l'eau en lame sur sa tìte. Ensuite, on passe en plein air devant une autre chnte d'ean moins considèrable. Tous ces bancs de roclıers sont plantés d'arbres aquatiques, de comouilliers sanguins, pistachiers cultivés, oliviers de Bolı́me, ronces a fleurs doubles, etc, etc, etc. Suirant le même chemin, on passe snr um pont qui fait voir le port M, destiné á mettre les gondolles à l’abri. De li, on cutre dans nne presquile plantée d'arbres les plus agréables, tels que les peupliers d'Italie, le saule pleureur, le peuplicr blane, le grisard. Des bancs variés procureront par leur position, des points de vue pour jouir du départ des gondolles. Le bittiment principal D, est posé sur un rocher escarpé, sur lequel on a plante, par groupes, des arbres agréables.

II. Ile de F́nus. Céle ile est plantée d'arbres isolés de toutes les variétés d'urbres aquatiques, tels que saules pleureurs, peupliers, faux trembles, penpliers d'Atheines, peupliers noirs, etc, etc. Ils sont 


\section{(21)}

disposés ça et là par nasse, arec des banes champêtres. Cette île se troure presquà fleur d'eau, de manière que, dans les grandes chalcurs, on va y respirer le frais, et de lia on monte au desert.

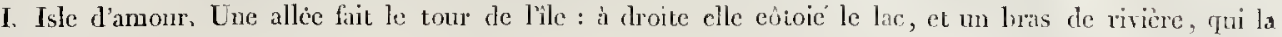

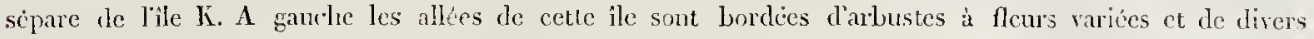
groupes d'arbres, eomme platanes, peuplicrs suisses, frünes pendans, eytises des $\Lambda$ lpes, cle. Deux ponts font passcr sur la petite île $\mathrm{K}$, et cette ile de l'Amitié est plantie, sur les hords re la rin iére, d'arbres aruatiques, comme saules pleureurs, saules marceaus, peupliers d'Athénes, peupliers du Cinada; prés de l'uu des deux ponts se trouve un pavillon chinois oú loon pourcait pícher à l'abri du uanais temps.

1. Monticule an haut duquel il y aumit une pagoile. Le chemin du bîtiment principal qui conduirait a cette pagode, scrait plante de groupes darbres très-touffus, en laissant des clairieres. Le vallon serait orné d'arbustes à fleurs pour se lier arec les autres groupes de terre de bruyère situés du côté de l'autre rive. On a placé derriẻre les arbustes et arbres, des banes qui laissent voir la riviére et l'ỉle. Cet endroit forme un bocage delicieux, pour arriver à la pagorle L. Le chemin qui prend naissance su diemin Y est montueux; arrive à l. pagorle $N$, on découvre une partic hu lac et des cmirons; il sert de point de vue pour le bâtiunent principal. Ce monticule a trente prieds d'élévation. Un autre chemin passe de même dans un bois très-touffu. A lissue le l’allée qui arrive á la rencontre du clıcnin qui le traverse, se trouve une clairière ì ganche, les arbres qui la bordent sont d'une teinte très-sonbre, et à l'extrémité est le tombeau diui ani révéré.

N. L'autre pagode est placée sur un monticule. Un scul chenin y conduit. Ce rhemin n’est pas aperẹu. Il se trouve parfois entourć d'arbustes et darbres isolés, comme vernis du japon, noyers, pacaniers, sumues, etc.; les parties de pelouse qui entourent ce inonticule, sont très-profondes, et deviennent un endroit très-sauvage; et ì lestrémité se trowe le tombeau de Confucis:.

O. Pagode placéc sur un roclıcr, doà l’ean sortant avec impetuosité, et de cascade en cascarle, irait tomber dans un immense lac.

P. Isle des Roclics. Cette île serait cutıe deux montagnes vomissant l'eau. Le soubassement de ce rocher à fleur d'eau aurait une issue pour entrer dins une pagorle garnio de glaces de tous cótçs avec des sophas au pourtour. Le plafond de eette salle serait en glace sans tain, sur lequel il serait établi un bassin dans lequel on mettrait des poissons rouges, dorès, et des oiseaux dans des globes qu’on placerait sur ce même plafont. On croirait voir les oiseanx dans l'eau. Des chemins pratiqués daus les roches montent it une autre pagode d'une hauteur supérieure, où se trouve le bassin dont on vient de douncr le détail.

Par le moyen d'une nuchiue hydraulique, on ferait monter les eaux qui, par l'établissement d'mne màcanique, mettraient en jeu $m$ orgue qui exécuterait plusienrs aiss.

Q. Chemin du désert, passant à travers les roches, parmi les sapias et les torrens qui coulcraient à Aroite et à gauche dudit rocher. La première pagole se tronve à vingt pieds d'clévation au-dessus du nivcau de l'eau; on arrive ensuite à une pagode plus élévéc de douze pieds, dont la vue domine sur tous les cuvirons. En suivant le mème chemin, on rencontre deux pagodes dont la derniere est eneore plus Clevéc que la première, puis en eontinuant, on descend dans une autre pagode. Apres a oir parcouru ce déscrt, il se trouve un clıcmin conduisant à un Elysće; cet Elyséc serait établi parni plusicurs monticules, lesquels seraient plantés d'arbres à fruits et à flcurs, tels que eanclia, azalía, rhododendrum, ete, afin quil $y$ ait toujours des fleurs on des fixits.

R. Clicumin destinc aux voitures et cavalcades, ì partir d'R en S. Le chemin monterait d'S cn T et redescendrait dans une vallée bordée de coteaux escarpés, dont les parties de roclics menacent ruine. De $T$ en $U$ le chemin est très-montueux. Arrivé à la lettre $\mathbf{U}$, on eommencerait à être dans une partie plantéc d'arbres fruitiers et d'arbres á fleurs, il ne resterait plus qu'une gorłe à parcourir pour arriver à l'Jilysée.

V. Différentes rivières seraient bordécs de rochers et d'arbres aquatiques.

$\mathrm{X}$. Rivière passant à travers un rocher dont les arbres paraitraicnt être retenus par des roches; et dans d'autres endroits, les roches sembleraicnt menacer ruine, ou ctte soutenucs par les arbres; divers torrens passeraicut entre les roches.

Y. Diffèrens chemins borderaient li rivière. On placerait des figures aux angles des massifs. 


\section{(22)}

\section{$\mathrm{N}^{\circ}$ 19. JARDIN FANTASTIQUEE ANGLAIS.}

C. Jardin était entouré de murailles qui masquaient la vue de la campagne; une source d'ean viye se trouvait sur la drnite, a six pieds d'élćvation, et ses caux élaicnt conduites an potager par des plombs.

Des rivières ont été creusées. Les terres ont été transportées sur lallée désignée $O$, élevéc de cinq pieds jusru'au temple desinné $H$; de maniére que l’on décourre toute la campagne de cette allée. On a eu soin de planter rà et lá sur les ềtés du chemin, de grands arbres et sur les talus des arbustes pour soutenir le ehemin et cu même temps eacher la muraille et les grands arbres, et mettre à l'abri du soleil.

Une partie des prairies a été creusée et les terres transportées sur les massifs, pour donner au terrain un mouvenent pittoresque ct agréable.

A. Cour d'entrée.

B. Battiment principal.

C. Basse-cour

D. Terrain élcvé ct soutenu par des rochics près le bâtíment.

E. Petit Hermitage servant de cabinet de travail; dans l'angle de lhermitage, on a placé une glace qui fait aperecroir le temple romain désigné $\mathbf{H}$.

F. Rocher sous lequel une source d'eau vive tombe par cascades de la hauteur de six pieds, dans un lae cntouré de peupliers suisses, de saules pleureurs, arbes à tiges, arbustes à fleurs et à odeur; une rivière sortant dn lac passe sous un pont clinois $\mathrm{N}$, et traverse la prairie devant le bàtiment.

La rivière de droite traverse la prairie, en passant sous le pont lollaudais 11 , chenin $O$, et forme Vîle de Bélus P, fils de Neptune; repasse sous deux ponts, l'un rustique et l'antre hollandais, et de la dans le lac $G$. La rivière regagne la piece d'cau F.

G. Tac entouré de saules pleureurs, penpliers d'Italie, platanes et fiènes pendans, peuplier banmier, etc.

H. Temple posé sur une éminence, ayant vue à travers les arbres sur la eampagne et sur une prairie plantéc d'arlıres isolés, eomme tulipiers, catalpa, mûricr de la Chine, frènes à fleurs; et, près du bàtiment, des acaéas triacanthos, et blancs. Le trop plein des caux passe sous le tcmple et va se jeter dans la prairie.

I. Chemin eonduisant à la salle $\mathbf{K}$ entouréc de figures posées sur des gaînes. Entre ces ligures sont placés des bancs elinois, enviromés d'arbustes à fleurs odorintes.

L. Chemin traversavt le bois, le pont hollandais désigné M et la prairie plantée d’arbres isolés, comme saules blancs, saules marceaux, frèves pendans. Arrivé à une figure représcitant Diane, on trouve deux elicmins, ccluí de droite fait traverser le bois planté d'arbres de moyeme hauicur. Ccux qui sont en avant dans la prairie, sont plus petits, et eeux plantés dans le fond, très-grands, de manière à faire croire que c'est une forêt très-étenduc. Arrivé an pont rustique, formé avec des branclıcs d'arbres, on aperçoit à droite des arbres isolés dans la praírí, avec un banc en gazon, plicé au pied d'un coteau, jouissant de la vue du temple de Bellone, désígné P. De l'autre côté, la prairie plantée d’arbres d’une autre nature, va rejoindre le chemin de ecinture, et suit derrière-le temple désigné II.

Liautre chemin passant dans une prairie, de lá sur le pont hollandais, eonduit dírectement au temple romain. Arrivé à ee temple, on a dix marches á monter. Sur la droíte du bútiment se présentent denx lremius : celui de ganche fait traverser la prairic, le bois, et va rejoindre le bàtiment; l'autre, l'allćc de ccinture, traverse le bois, conduit à un superbe espalier et à lansle du mur. Une chaumière, désignée $Q$, sert de lieu de repos, En suivant ce ehemin, on arrive au bâtiment.

R. Potager, avee des plate-bandes, garnies de quenouilles, entremẻlées de pommiers de paradis. 


\section{No 20. JARDIN FANTASTIQUE.}

Ce Jardin contient environ douze arpens.

A. Cour en avant du bâliment et une pelousc en gazon cntourée de fleurs.

B. Remise, écurie et basse-cour.

C. Chàtcau.

D. Lac arec deuy îles réunies par un pont.

E. Chemin traversant la prairic et la forêt, et allant au rocher F, avec un pont chinois. L'eau tomberait d'un rocher de dix pieds, dans un petit lac, et se répandrait dans la prairic, par deux petits bras de riviere; ensuite après avoir traversé la forct et la rivière, on arriverait au temple de Dianc $G$, posé sur une glacière; puis on passcrait sur un pont qui conduirait au lsàtment $\mathbf{C}$.

I. Chemin conduisant au vallon où il y a un rocher sur lequel il se troure un temple $\mathrm{K}$, dont. l'escalier a été pratiqué dans les roches; différens petits elrewins ont été ouverts dans la pelouse ou est le temple chinois $\mathbf{K}$, lesquels ne sont pas aperçus du bâtiment.

J. Vallon.

\section{$\mathrm{N}^{\circ}$ 2т. JARDIN CIIAMPÊTRE.}

\section{Ce Jardin contient envir on scize arpens.}

A. Porte d'entréc, arec deux pavillons pour le portier; le chemin en face conduit au batiment B, il est bordé de grauds arbres : ce bâtiment est établi sur un terrain élevé de cinq pieds an-dessus du niveau de la porte d'eutrée, et domiue sur une grande partie du jardin. Du bâtiment on a pour point de rne le pont hollandais $T$, un grand lac, une prairic émailléc de fleurs et plantée de groupes d'arbres isolés, tels que platanes, peupliers d'ltalie, noyers, pacaniers, tilleuls d'Amérique, peupliers, trembles, etc $\Lambda$ droite du bâtiment est une grande prairic, dans laquelle sont placées de grandes masses d'arbres et plusieurs pièces de céréales, prés desquel!es se trouve le temple de Cérès clésigné $\mathrm{Q}$, déesse des moissons et des champs, fuisant point de vuc au bàtiment; sur la même prairic, est établie une statuc V, repuésentant Pomone, déesse des fruits, autour de laruelle sont des masses d'arbres à fruit; à gauche du buttiment, est une prairie qui se lie par une jente douce au lae, dont les caux passent sous un pont rustique, trarersent le potager, et vont arroser la campagne voisine. La pelouse, en face du bátiment, du côté de la porte d'entrée, est plantée de masses d'arbres très-touffus, et dequelques arbres isolés; à l'extrémité est un ha-ha, par dessus lequel on aperçoit la campagne, qui se lie avec le jardin. Le chemin de droite, en sortant du batiment, passe sur le pont $T$, conduisant dans l'île de IAmitié I, au centre de laquelle est le temple du mème nom, qui pourrait servir de salle de bain; cette île est plantée de groupes d'arbres isolés, tels quérables sucres, érables á feuilles de frênes, hêtres pourpres, nragnolia, alisiers, sophora du Japon, etc. Ensuite, passant sur le pont chinois $\mathrm{N}$, on traverse une prairie plantéc de grandes masses d'arbres, sous lesquels sont placés des bancs pour se reposer; en continuant, et passant sur le pont $\mathbf{M}$, on arrive au temple de Téuus $\mathbf{L}$, posé sur un rocher d'où l'eau sort avec impétuosité entre les roches, et retombe dans les riviéres qui scrpentent autour de la prairie; le pourtour du temple est planté d'arłores isolés, tels que bonducs, bouleaus, liquilambars, micoconlicrs, ctc.; cu suivant le chemin on côtoie la rivière qui, tantút est caclı́e par des masses d’arbres, et quelquefois se trouve a fleur d'eau, suivant le mouvement du terrain : on arrive à la salle de danse $\mathbf{K}$, antour de laquelle sont des bancs de gazon, plusieurs arlures isolés sont plantés cà et lí dans la salle de dause, on passe sur le pont rustique $V$, autour duquel sont plantés des arbres isolés, à travers lesquels on aperçoit la rivière qui serpente dans une prairie, plantée d'épines à fleurs doubles, sorbiers des oiseaux, pavia à fleurs jaunes, etc.; cn continuant, on arrive au batiment B; sortant de la salle de danse, et prenant le chemin de droite, on parcourt une partie d'un bois, au bout duquel, 


\section{( 24 )}

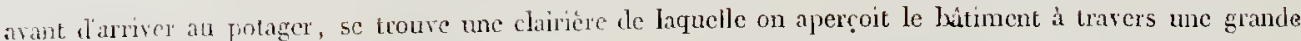
cuantitéd arbres isoles, dont les brancles portent sur la riviere et le lac

II. Barhe , dass laquelle m cultive des myrtes, des halmia, rhododendrum, acwia, minosa et antres.

D. Yerger.

F. Bassin entouré de gitzm.

G. Orangeric

E. l'utaser.

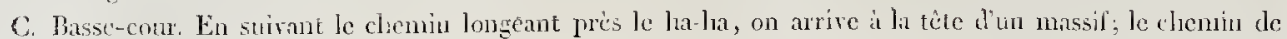
droite traverse un grand massil d'arbres, et conduit an temple de Fabins $\mathbf{S}$, fils d'flcrcule, posé sur un teriain besé de quatre pierls au-dessus du nivean de lia porte d'entrie. Desant le temple est me prairie, plantée d'arbres en massifs et isolés; sur la droite sont plantés des sapins, pins d'Ecosse, pins du lord V/citmontli; et a gauche, des érables jaspés, acacias, triacanthos, cte. Le chemin de gauclie, conduit à la salle des GrandsIInmmes R : celte salle est entource de grands massifs d'arbres, et autour sont iles banes pour se reproser'; en sortant de ladite salie, on rencontre deus chemins : cclui de droite conduit au cliemiu de crinturc, l'autre conduit à une tête de massif; le clıcmin de gaurhe conduit au temple de Cérés, désinné Q, et colui de droite traverse le bois, et conduit au baituncut russe P, duquel on aperçoit des masses d'arbres parni lés cérézles, el le temple de Cérès. En sortant du temple russe, deux chemins se présentent : celui de gauche conduit au bàtiment B, aprés une promenade extrêmement variée; celıi de droite conduit ì lobelisque O, placé sur mu tertre, entouré d'ubbes isolés : ce monument est apereu de divera nudroits a travers les arbres et les clairieres.

\section{No 22. JARDIN SYLVESTRE.}

Ce Jardin contient environ trente-six arpens.

A. Bitiment du proprétairc, ajant pour point de vue un obélisque $\mathbf{T}$ et le temple rle Neptune $\mathbf{R}$, posé sur un rocher tris-escarpé, doù l'eau tombe de roche en roche dims diflétens lacs. Plusicurs chemius conduiscnt dans diverses groltes dans lesquelles on a mis de la mousse et des graces pour répéter les eaux qui tombent, soit sur le devant du rocher, ou intéricurement dans bir grolte. D'autres chrouins fratiqués a travers les rocliers, les pius, sipins, conduisent a différentes allées du jardiu.

Sur la droite du bitimcnt, on a pour point de vue, à travers de trés-grands arbres estiraux, le temple de Diane, déesse des forêts, désigné $\mathrm{N}$, posé sur une éminence triés-élcréc.

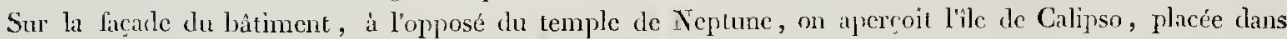
un lac, entourée de saules pleureurs, peupliers d'Ttalic. A l'extrínilé du lac, an pont $G$, il y a une trísbelle châte d'ean. Les eaux traversent une prairie plantéc d'arbres fritiers, et eoulent á travers le potager.

Promenades du bitliment au jardin.

Deux clemins se présentent ; celui de gauche traverse une forêt plantéc de maronniers isolés sur la prairie, a travers lesquels on aperçoit des peuplicr's épars çi et lí. Le pourtour de lia muraile est planté d'arbres tries - touffus pour la caclıer. Arrivé al l'obélisque, deux chemins se présentent; celui de gauclic passe audessus du temple de Neptune; un pont fait passer au temple. De lí, on voit Lomber l'eau de cascades en cascades jusques dans le lac. Liantre chemin passe à travers les roclies, de la hauteur de douze pieds; et à l'opposé, on voit l'cau passer sous les pieds, et tomber de roclie en roclic dans différens lacs; de lit, on monte par unc pente douce et on arrive au chemin de ceinture. Ce clımin conduit à la maison du bûcheron, désigné $Q$, en avant de la petite fabrique. On y a planté des arbres fruitiers.

Le chemin de droite du bâtiment, fai passer sur un pont rustique; le là se voient trois chûtes d'eau, et une chùte sous le pont, et l'on apercoit la rivière serpenter dass la prairie. Du front, on se rend à la salle de: Jugatinus, P, Dicu du sommet des montagnes; on l'invoquait aussi quand les nouveaux mariés se juraicut Ja foi conjugale.

Quatre chemins se rósentent ; celui de gauche va rejoinlre le clicmin de ccinture et, de lá, la cabane du bùcheron. 
Le chemin de droite traverse des massifs d'arbres isolés sur la prairie; an milicu d'eus se trouvent des bancs champétres. Ce chemin va rejoindre un angle le massifs. A droite, on va au chemín de ronde; lautre remonte par un chemín trés-escarpé, et va au temple de Diane $\mathrm{N}$.

Promenade du côtó dn lac. Deux chemins se présentent. Cclui de droite fait passer sur un pont clinois, parmi de trís-grands arbres, vus du bâtiment. En suivant. ledit clıcmin il conduit à travers les arbres, et prís dı bord de l'enu, í un des ponts de l'ỉe; l'autre chemin fait arriver à m carrefour, et en le continuant, on va rejoindre celui de ronde.

Un autre monte trés-rapidement au temple $\mathrm{N}$, et celui de ganche à la salle P.

Lc chemin de gauche du bâtiment passe au milieu d"immenses peuplicớs, plantés sur le gazon, et à travers lesquels on apercoit le lac. Sur la gauche du pout se trouvent différentes allèes qui trayersent un fleuriste désigné D, avec un bassin au centre et un superbe espalicr, arec une serre tenpérée, et derrière sont placées des remises et écuries désignées B. En suivant le cliemin, on traverse une plairie plantée d'arbres f́ruitiers; arrivé à l'angle du bois, on a placé un banc champître, et í travers les irlores fruiticrs, on voit une clute d'cau du pont en roche $G$. En suivant le même chemin, on passe sur un pout rustique fait avec des trones et branches d'arbres. Passé ce pont, on tronve deux elıemins; celui đle fauche conduit à l'île d'Anour, plantéc d'arbres aquatiques, plantes étrangéres, comme azalea, halmia, rhorlodendrun, toutes les espèces de roses, et autres, ce qui lie le fleuríste arec lïle. A la tète de l'ile, est le temple de l'Amour, et à l'antre extrómité, la figure de Flore. S'autre chemín conduit à plusieurs autres; le premier a droite, firt traverser une garenne L entourée d'ćchalats trés-resscrrés, conluit au potager et vá rejoindre le clıemin de ronde. En quittant le pont $G$, un clıemin trarerse la forêt; un autre conduit au chıemin de ronde; nun autre va rejoindre les différentes fabriques $\mathrm{N}, \mathrm{P}$.

IH. Potager entouré de fossés avec plantation d'arbres fruiliers.

I. Orangerie, avec deux pavillons aux deux extrémités, et une terasse sur laquelle on place les orangers pendant l'été. Au bas de la terrasse, on a placé des chảssis pour les melons, el autres. Derrière cette orangcrie est un petit bâtiment, dans lequil on place les instrumens aratoires; des dépòts de fanaier et poleries se trourent derrière la grande serre.

Un chemin de ronde passe derrière les dépóts de terres: on a eu soin de bien masyuer la muraille.

\section{$\mathrm{N}^{\circ}$ 25. JARDIN PASTORAL.}

Ce Jardin contient soixante-six arplens environ.

A. Bâtiment gotlique, avec quatre tourelles, entourć d'eau limpide, provenaut de différcus ruísseaux, produits par les rivières et les lacs; ils traversent la prairie et vont serpenter dans celle qui est au bout «lu jardiu,

Les deux tonrelles et une partie du bàtiment ont pour point de vue la bergerie hollandeíse, désignéc $\mathbf{F}$. Il se trouve à l'cntrée de la forêt un autre point de vue. A travers dimmenses peupliers, on apercoit un moulin à cau, désígné E. Sur la facade de gavuche du bâtiment, on voil lî̀le de Cérès $\mathrm{S}$, arec plusicurs ponts et la campague qui se lie avee le jardiu. On a cu soin de planter antour dn batiment de trés-grands peupliers suisses, dont la teinte est trés-forte en coulcur, pour faire repoussoir. Les lács sont bordés de pelouses et d'arbres isolés. Les prairies, eı face du bàtiment, sont plantées d'oseraies, pour ne pas masquer la vue du château. Sur le côté des saules pleurenrs, des peuplíers d'Italie, des bouquets d'arbres aquatiques, variés par leur port et leur lanutcur.

Sur le devant dn bàtinent, du côté de l’ntrćc désignée T, est une prairie par-dessus larguelle on voit les voyageurs qui passent sur la route, et à langledu mur sur la droile, est une figure représentant l'Amitié. De l'autre còté ce sont les remises et écnries $\mathbf{X}$. Contre les reníses est adosséc une très-belle scrre d'orangerie $U$, et sur le devant des clıḋsis est un potager V, dont les murailles sont garuics d'arbres fruitiers.

Promcnades. Un parterre, planté d'arbustes les plus rares, se trouve placé dans l'île de Cithére (), cn face du bâtiment. Deux ponts-lcris ferment cette île. On passe sur le pont qui est en face de la bergerie F. Deux chemins se présentent. Celui de droite conduit sur un pont gothiyue en jicrrc. On troure deux autres chemins. Celui de droite mène dans le bós, et conduit à une salle B, où sont deux volières, entourées de bancs champêtres, 


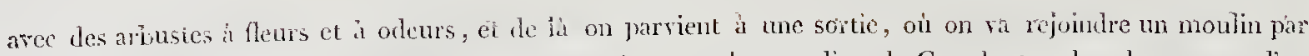
différens ponts. Du chemin qui est in face, on passe i travers les preupliers du Canada et saules pleureurs, ef lon

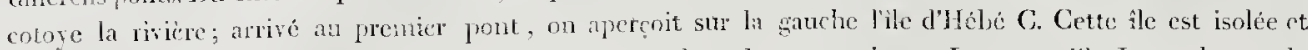

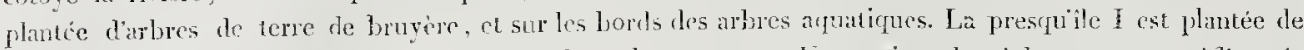

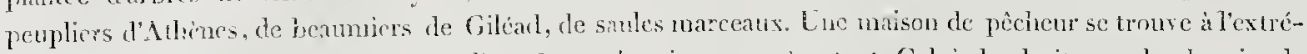

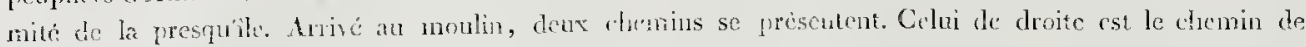
reinture, bien bordi diabres desinces á cacher les murailes, et de l'antre coté, des arbres isolis par masses,

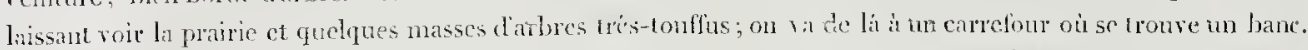

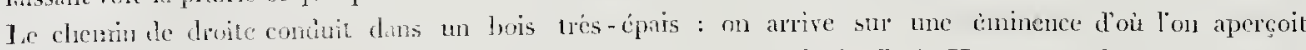

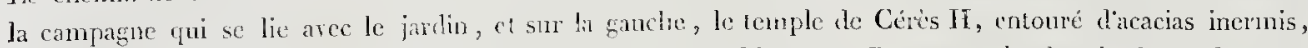
glutinosi, írables siconores, érables sucre, peupliers trembles, c'te. Reprenant le cliemin rlevant le pont, on traverse le bois jusquau carrefour, ou se uoure wi bane de verdure, qui a pour point de vue une imnense prairie, plintéc darbres par masses, et à l'cxtrénité le temple de Cérés. Le cluemin de droite conduit à zue maison de bnelieron G, cutouré de leètres, cliênes, érables, etc. Le chenin de gauche traverse la force ef va rejoindre le lıa-lıa, ou saut de loup, d'oì l'on apercoit arec surprise une immense campagne qui se lie aree le jarlin. On a soin de séloigner du hal-lia, pour wi pas voir les murailles. En continuant la route, on troure deux cliemins. Cilui de droite conduit à une maison de garde, qui a une Irès - Joclle vie sur la campagne. Cette maison cst entourée d'arbres, tels que tremblés, tilleuls, boulcaux, cte. Ce mème chemin conduit à une porte gothique $\mathrm{N}$, qui sert de point de vo pour le bàtinent et en mêre temps ficunce aus voitures pour l'exploitation du jardin. Sur la sauche de lit porte grothique, est une forte en bois hut, qui sert de fermeture à mu hernitage $\mathbf{I}$, plautée d’arbres fruitiers, de rignes, de céréales, de ligumes, de fleurs, aree des parties de gazon, banes clampêtres, petit huis planté d'arlores d'agrément, comme c) tises les Mnes, mérisiers à fleurs doubles, ćpines loses, sorbiers des oiseaux, acacias roses, arbustes, lilas, syringats, rosicrs, althea, etc. Ces arbres seraient plantés seulenent dans lintéricur et à l'extérieur. de grands arbies foresticus.

M. Retraite arec bane de gazon autour. Après avoir parcourn tous les chemins de l'hermitage, on tronve, au rentre, use chapelle gothique qui sert de lieu de repos. Le cliemin en face de la porte gothique traverse la forêt, la prairic, ardite í la bergerie; en ouvrant la porte, les noutons, les vaches, les clevaux se répandent dans la prairic, ce qui fait un tableau cliarmant : en continuant la route on arrive au bâtinent.

Le chenin de droite de la porte gothique traverse le bois et les arbres fruitiers. Arrivé à un angle, le chenin de droite fait traverscr les arbres fruitiers, les céréales; conduit à un point rustique, de lí traverse une jrairic plantéc d’arbres isolés, comme saules pleureurs, peuplicrs d'Jtalie, peupliers d'Athénes, etc.; sur la droite de la prairie est un bras de rivière, sur la gauche du pont, est un autre cliemin qui borde la rivière, le. lac, ct ra rejoindre le bảtiment.

Le chemin de droite du pont traverse deux presqu'iles. On a, du pont $\mathbf{R}$, le point de vue du bâtiment et une large rivièe; et de l'autre côté, la vae d'une rivière, arec un pout qui sc trouve a l'extrénité du jardin, et un ha - la qui laisse voir la campagne, qui se lie arec le jardin, plus la riviere qui serpente dans la prairie extérieure. En quittant le pont, deux chemins se présentent : l'un conduit an potager $\mathrm{N}$, et lautre est le chemin de ronde, traversant la prairie et allant au bâtiment.

\section{$\mathrm{N}^{\circ}$ 24. JARDIN ROMANTIQUE.}

Ce Jarilin contient trois cents arpens environ.

A. cliâteau gothique. Ce bàtiment se troure placé à mi-còte.

Le clấteau a pour point de vue, du cóté du midi, le village $\mathbf{J}$, le temple de Vénus $\mathbf{M}$, et le temple re Neptune H.

$\Lambda$ gauche du bâtiment, on a pour point de ruc la porte gothique désignće D; plus deux clutes d'eau tombant sur le cóté dlune prairie émaillée de fleurs arce des arbustes sur le bord de la prairic pour le premier plan; sur le sccond, des arbrisseaux; sur le troisiène, de petits arlures a fleurs, et sur 


\section{$(27)$}

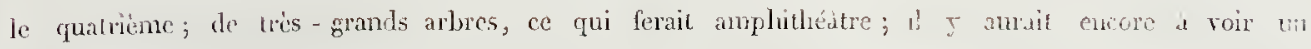
obclisque $\mathrm{S}$.

Te cóte à droite, a jour point de vue deux ponts, un lac, unc prairie émailléc de fleurs; sur le devant d.s lac re sur les denx cólés de la prairie, des arbres isoles et le hort des rhemins planté räbres tres-touffus. e.t par fuis, des clairières laissant apercer oir la prairie, et à l'extrémitè un moulin N dont les caux tomberaimi de six preils sur des porhers qui feraient jaillir leau; et le soleil donnant sur ce point, ferait un effet adnirahje.

Le còle du bitinent au nord, a pour point de vue l'ile dAmour, désignée $Q$, aver mo figure reps -

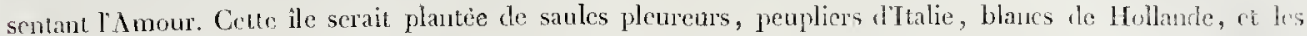
rheunins hordés d'arbustes à fleurs et à odeur, plantés par masses, et de manicre a ne pas apercevoir he diemin oppose.

Lîile de Flore $N^{\top}$, plantẻe d'arbres les plus rares. Ces deux illes se trowreraient placèes dans un trèssrand lac, dont les caux traverseraient un rocler et iraicnt se précipiter dans un lae immense.

B. Polager de quatre arpens avec serre chaude el chissis.

C. Basse-cour, temise, écurie et logenent du jardinier.

D. Porte d'entrée gothique.

E. Chemin conduisant au bâliment $\boldsymbol{A}$.

F. Clicuin conduisant à la salle des Gramls-Hommes.

G. Chemin conduisant à la bergerie; vácheric L. En continuant le mème clıenin, traversauı la forêt, on rencontre divers monmens gothiques, tels que tombeaux, vases; et cusuite on arive au temple de Neptune 1 .

H. Temple de Neptune posé sur un roeher d'oú il sortirait de l'eau qui retomberait par dilférentes roches dans un lac plus bas de trente pieds. Autour dudit roclier, il y serait pratiqué différens clıenins sinneux, plantés d'arbres verts. Dans lesdits chemins, il g cn aurait qui descendraient sous la voûte du temple, ed d'autres remonteraicut vers le chemin qui fait le tour de la propriété

1. Temple ruiné aperẹu du village.

J. Village an milieu duruel il y aurait un hermitage qui ferail point de vue au chìtean; ce village scraí, planté d'irlures d’agrẻnent, et toutes les frersonnes cmployées anx travans de l'agriculture y seraient logèes.

K. Temple de Diane, servant de point de vue au temple de Neptune.

L. Bergerie et vacheric faisant point de vue au battiment, et derrièe cette bergeric on établirait des parrs pour les dilfèrentes espéres de moutons.

M. Temple de Vénus.

N. Moulin a eau, dont la clute serait environ de six pieds; l'eau tomberait dans un lac, oc qui ferait point de vue au bâtiment.

O. Temple de Pomone, décsse des fruits, entouré d'arbres à fruits.

P. Logement du cultivateur.

Q. Ile d'Anour, plantée de peupliers, saules pleureurs et autres.

R. Ile de Flore, où on planterait toutes les espéces d'atbres à fleurs éa et là dans la prairie on planterait des saules, des peupliers, des arbres et arbustes qui viennent dans la terre de bruyére.

S. Obélisque placé à la rencontre de plusicurs allces, et faisant point de vue dans la promenade.

T. Salle irrégulière où il y aurait divers monumens tels que tombeaux golhiques ct autres.

V. Salle de danse, d'escarpolette, jeu de carrousel, etc. 


\section{$\mathrm{N}^{\circ}$ 25. JARDIN, PARG OU CARRIĖRES.}

Ce Jardin, contenant sept mille cent quatorze arpens eniron, est compose des pares de Versiniles, Trianon grand et petit, d'un jurlin sylvestre, pastoral, champilie; romantique chinois et ronantique franrais.

I. Clıàteau de Versailles et dépendances.

2. Jardin avec pieces d'caux, boulingrins, bosquets, ete.

3. Grand tapris vert, orné de figures.

4. Ile dimour.

5. Bassin de Neptune.

6. Grand canal.

7. Grand pare et la faisanderie.

8. Ménagcric des animaux féroces, avec volicre.

9. Ménagerie des animaux paisibles.

zo. Ménagerie des cerfs.

ז. Potager-marajcher.

12. Jardin pour la naturalisation, et pépinière.

13. Grand quinconce; jardin pour les jeux; balauçoire; jeux de bagues; salle de danse, escarpolette.

14. Immense jardin pour les pépinières nationales; jardin de botaniçue; scrires chandes, etc.

17. Potager clu Roi.

18. Quinconce d'arbres fruitiers. Toutes ces parties conticnnent environ quinze cent un arpens.

15. Jardin mglais, Petil-Trianon, contenant environ quatre-vingt-donze arpens.

16. Jardin francais, Grand - Trianon, contenant environ cent onze arpens.

JARDIY SYLVESTRE. Ce jardin contient curiron neuf cent soisante-dix-huit arpens; il est bordé par le grand parc de Versailles, à droite par l'un des fossés, et à gauche du point $\mathbf{A} \Lambda$, en suivant le chemin $\Lambda \mathrm{B}$.

A. Bàtiment principal, ayant pour point de vne le temple de Cérés B, entouré d'arbres fruiticrs; et une arès-bcile prairie.

C. Temple de l'Amitic, cntouré d'arbres ì fleurs et à odeur.

D. Ancienne faisanderie H. Colosse de Rhodes, servant de point de vue au clıtiteau, et pour la navigation.

II. Temple de Nérée, Dieu marin, fils de lOocéan.

G. Temple des Nayades, filles de Jupiter. Elles présidaient aux fleures et aux fontaincs.

E. Temple de Messapus, fils de Neptune.

I. Entrće d'une carrière, passant sous le chemin; clle cntre dans nn lieu très-profond : les extrémités dés chemins sont plantés d'arbres rerts. Différens chemins se croisent, et vont traverser une immense carricur, au-dessus de laquelle est bâti le temple de Jupiter, désigné $\mathbf{K}$; différens chemins traversent une forèt inmense, des prairies, des céréales, des arbres fruitiers. Il y a aussi des lacs, des chutes d'cau, des ponts clinois, liollandais, ete.

JARDIX PASTOR $A L$. Ce jardin contient environ neuf cent cinquante-cinq arpens, il est bordé par le jardin sylvestre, et de lautre còté du point $A B$, passe près du village $M$, en continuant le chemin $A D$, jusquau point $\mathrm{A} \Lambda$.
A. Bàtinent.
B. Moulin it eau.
C. Ferme avec unc bergeric, pâturages.
D. Rivière souterraine,
L. Hermitage.
F. Cabanc de bucheron.
G. Temple de Nodinus, Dien qui frésidait aus moissons lorsqu'elles germaient. 
II. Temple de Napées, nyimphes qui présidaient anx prairics et aux bocages.

I. 'Temple de Cérès, déesse des moissons.

L. École militaire de Sint-Cyi:

M. Callianasse, nymphe qui présidait à la bonne conduite, à la décence des noenes.

JARDIN CICAMPETPE contenant environ huit cent soixante-neuf arpens; il est bordé à droite par le chemin $\mathrm{AD}$ et à gaurke par. le chemin $\Lambda \mathrm{E}$.

A. Bitiment.

B. Prairie plantéc d’arbres d’agrément, d'arhres fruitiers, viggnes, cérćales, forèt; et ì l'extrémilé du jardin, des moulins à vent.

C. Temple de lomone, déesse des fruits.

D. T'emple d'Andréas, dien qui présidait à la maturité des grains.

E. Anthée, fancux géant, fils de Neptune et de la Terre.

F. Ecluse pour descendre les gondoles et bateaux dans les parties basses des jardins, et pour passer sous le colosse de Rlıodes, et de là au grand canal.

G. Chutes d'eanx de la houteur de quinze à vingt pieds, dians différens lacs; ponts rustiques, ponts gothicques, arec divers monumens placés à mi-côte, doì on roit des prairies, des masses d'arbres verts, plantés sur les roches, avec des cabanes de bûcheron, cliamnières, etc.

H. 'Temple d'Arginns. C'était un Grec qui se noya en se baighant. Aganremon qui l'ainait beaucoup, fit bâlir en son honneur un tenple qüil dédia à V́ćnus.

L. Village de St,-Cyr.

JARDIX ROHANTIQUE CHINOIS contenant environ nenf cent trente-trois arpens ; il cst bordé à droite par le chemin IE, à gancłse par le fossé, ensuite par le chemin AF et le pont $A \Lambda$.

A. Bítiment shinois, placé en face d'un immense lac, entouré par fois de rochers très-escarpés; des allées souterraines de montagnes très-élerées, sur leaquelles il y a des pagodes, des antres, des précirices, des riviéres souterraines, des ponts, des obélisques, des fonlaines, des statues, des figures, des prairies émaillées de fleurs de tontes les saisons, des tombeaux, des ruines, des banes rustiques, tures, chinois, etc.

13. Ponts.

C. Immense lac.

D. He de Triopus, fils du Solcil; il donna son nom it un promontoirc, et à ane ville de Caric. Différentes allées suivent le bord de la rivière et da lac $\mathrm{C}$; d'autre traversent de grands massifs d'arbres et passent sous plusicurs rochers sumontés de pagodes.

F. Ile des roches. Dans cette ile se tronve une pagode, sur une montagne très ćlevée cntouréc d’arbres.

K. Ile de Canope, divinité égyptienne, dont les prètres passaicut pour de grands magiciens. Cette île cst traversćc par plusieurs cliemins bordés de grands arbles, avec plusieurs monumens chinois.

Les artistes chinois dislinguent trois différentes scènes auxquelles ils donnent les noms de riantes, d'horribles et d'enchanteresses. Cette dernic̀re dénonination répond à ce quon nomme scène de romans, etc.

JARDIN ROHANTIQUE FRANCAIS contenant environ scize cent soixante-quinze arpens ; il est bordé d'un côté par le chemin AE, le point AA, par le grand parc de Versailles, et le fossé du côté de Satoris.

A. Bảtiment principal, faisant point de rue au château de Versailles. Ce bâtiment est entouré d’arbres, ayant point de vue sin une immense prairie : une riviere tombe de cliute en clute dans un grand lac.

B. Ferme de Satoris, ayant quatre cents arpens. Cette Ferme est entourée d'arbres à fruits, de prairics, d'une rivière et abreuvoir pour les bestiaux.

C. Lac immense, qui alimente la pièce d'cau des Suisses : au centre du lac, est unc île plantée d'arbres aquatiques; an centre de l'ile, se trouve le temple de Bel ou Bélus, fils de Neptune et de Lybic, et roi des Assyriens. On rendait les honneurs divins a sa statue.

D. Temple de Diane.

E. Temple de Pomone.

F. Lac reccrant les eaux par chute, provenant du lac clésiģié C.

Au centre du lac, cit le trmple de la Paix, posć sur un rocher ct planté darbres verts. L'eau 


\section{(3o)}

tombe, sur la gauche, de vingt pieds; sur la droite, de quarante pieds; une troisième clute se jotte dans un immense lac, au nilieı dıquel est l'ìle de Minerve, autrement Pallas, déesse de la guerre, de la sagesse et des arts, fille de Jupiter, avec le temple de la Déesse.

Le dessous du temple de la Paix pourrait servir d'usine pour la formation des eanons de fusils, etc. Des chemins pratiqués à travers les roches feraient descendre dans différens souterrains : l'eau tonıberait sur des rounges qui feraicnt mouroir des objets nécessaires à la confection de ces canons.

G. Teinple en ruine.

I. Obélisque.

K. Falacer, dieu des arbres fruitiers.

L. Temple de Fabulinus, dieu qu'on invoquait quaud les enfaus cormmençaient à parler.

M. Calchas, faneux devin.

N. Cantlos, fontaine proche de Namplie, oiı $\mathbf{J}$ unou venait se baigner tous les ans pour se purifier.

\section{VUES DU JARDIY, PARC OU CARRIERES}

$N^{\circ}$ r. Yue du clıâteau de Versailles avec une partie du jardin.

$\mathrm{N}^{\circ}$ 2. Vue du jardin sylvestre.

$N^{\circ}$ 3. Vue du jardin pastoral.

$N^{\circ}$ 4. Vue du jardin champètıe.

$N^{\circ} 5$. Vue du jardin romantique chinois.

$\mathrm{N}^{\circ} 6$. Vue du jardiı romantique francais.

\section{$\mathrm{N}^{\circ}$ 26. JARDIN D'AGRÉMENT.}

Ce jardin contient un arpent trois quarts.

A. Escalier pour descendre au jardirr.

B. Pavillon.

C. Autre pavillon.

D. Pelouse bordée de rosiers de toute espèce; les plus lauts au centre, les moyens et les plus petits sur le devant.

E. Massifs garnis de rosiers sur le devant et d'arbustes sur le derrière, qui ne s'élévent pas plus haut que six à sept pieds; les autres massifs, garnis d'arbres plus élevés, afin de former un amphithétitre.

F. Chemin conduisant sous le pont, sous lequel il y aurait des bancs de repos; le même elıenin conduirait à la salle de spectacle $\mathrm{H}$.

G. Pont et chemin passant sur le pont.

H. Salle de spectacle.

I. ct J. Salles de repos, avec une table au milicu pour mettre les rafraichissemens.

K. Temple des Muses.

L. M. Cabinets destinés pour l'habillement des acteurs et actrices.

N. Salle de repos, avec un banc de gazon, ayant pour point de vue le temple des Muses.

O. Petit pavillon chinois.

Il y a trois figures placées aux anğles des massifs.

La pelouse a été creusée de trois pieds, et les terres ont été transportées pour former les culées du pont et pour élcver les massifs sur lesquels le pont est établi. L'alléc bordant la salle de spectacle a été creusée à deux pieds en face le pont, et se réduit á zéro à l'extrémité de larlite allée; on a ètabli trois marches pour monter à la salle de spectacle; et sous le pont, on a formé un puisard pour recevoir les eaux de pluies.

Le propriétaire, liomme de goût, voulut avoir un jarlin oú il y eut diflérentes dispositions pour les arbres et arbustes, qui demandent les uns le soleil, et les autres le nord, des parties élevées, des parties basses, des allćes bien caclıces, des fabriques, unc salle de spectacle et des chemins fiaciles. 


\section{$(31)$}

\section{$x^{\circ}$ 27. JARDIN D'AGREMENT.}

Ce jardin contient deux arpens environ.

A. Bâtiment ayant pour point de vue le temple E, posé sur ton montícule; entouré d'arbres isolés : une serre d'orangerie, et une figure représentant Diane.

R. Pelouse plantée d'arbres d'agrément; le devant du bàtiment serait garni de fleurs, et sur le devant des massífs on planteraít des arbres verts.

C. Salle de repos et bancs de gazon entourés d'arbres d’agrément.

D. Chemin traversant des massil's d'arbres ísolés, plantés sur le gazon et allant au tenple d'Euterpe.

F. Chemin conduisant du batiment à travers le bois trés-touffu, à la salle $\mathrm{G}$, oì il y aurait un jeu de bague entouré de bancs de gazon,

H. Serre d'orangerie avee vestibule. Différentes ligures seraient placécs aux angles des massifs.

On suppose ce terrain plat, entouré de murailles.

Pour lui donner un mouvement agréable et le faire paraître plus grand, il faudrait creuser, en face du bảtiment, la prairíe de trois pieds au deux ticrs de distance du bàtiment et terminer à géro aux extrémités des allécs; les terres seraient transportées sur les allées et les massifs, le long des murailles, pour les cacher à la vuc.

Les massifs, á droite et á gauche, seront plantés d'arbres les plus hauts, avec des arbustes très-toulfus, comme lilas, syringas, sureaux, sainte-lncíe, arbres de Juckéc, sumacs, etc, de manière à ne pas laisser apercevoír ni les muraíles ni les personnes qui passeraient dans les allées bordant la prairie : celles-ci seraient plantées tantôt d'arbres isolés, tantòt de massífs très-touffus pour varier les? scènes et faire repoussoir aux différentes fabriques. D’ailleurs, il cst des arbres qui demandent de l'air ct à être isolés, comme catalıa, elc.

\section{$\Lambda^{\text {To }}$ 28. JARDINS D'AGREMENT.}

Ces deux jardins contiement l'un deux arpens un tiers, et l'autre trois arpens; ils ont été établis sul' l'emplacement de carrières qui laissaient de grands vides vers le milieu et en face du bâtiment. Pour éviter la dépense, jaí cru quil fallait placer des ponts, pour lier les buttes ensemble ct profiter de cette espèce de cahos, ce qui donne lueaucoup de variété dans ees jardins.

A. Cour d'entrće du premier jardin.

B. Seconde cour, séparée de la première par un petit mur d'appü.

C. Bâtiment quí a pour point de vue une prairie émaillée de fleurs, et un pont rustique, sous lequel on doít apercevoir la campagne. Ce pont est entouré d'arbres, arbustes à fleurs et à odeur.

D. Pelouse garnie de fleurs, et sur les côtés des massifs d'arbres d'agrément.

E. Chemin montant par une pente douce par laquelle on arrive à la tête d'uu massif. La figure de l'Amour y est placée. Le chenin de droite descend, et à l'angle du mur est un banc rustique fait avec des branches d'arbres. Le premier chemin qui se présente passe sous le pont. Hais avant d'y arríver, on a sur la gauclıc un vallon et à l'extrémité une ligure représentant Diane; autour de cette figure est un bane en gazon. En suivant le chemin qui conduit au petit pavillon $\mathbf{L}$, on trowe un autre vallon dans lequel il y a une ligure représentant Egyptus; et à l'angle du massif, est une autre figure représentant Electre. De la figure de l'Amour, on va au pont $\mathbf{F}$, on traverse le boís, et on arríve a la salle $\mathbf{G}$, salle de repos plus élevée que le pont $\mathbf{F}$, entourée de bancs et de grands arbres pour y être à l'ombre.

H. Chemin descendant sous le pavillon L.

K. Chenin descendant au pavillon $\mathrm{L}$.

M. Chemin conduísant diu bàtiment à la salle N. Cette salle est entourée d'arbustes et d'arbres à fleurs et à odeur, avec bancs en gazon. En suivant le clienun ou trouve une prairie avec des arbres isolés et rares.

A. Cour du densiéme jarlin 
1). Pátinent ou il y a trois marches pour atescentre dans le jardin.

C. Pelouse flantie d’abres, arbustes et nuassifs de fleurs sur le derant du bàtiment. On a trois points de we, le pont $\mathrm{F}$, lit salle $\mathrm{G}$, et le pont en face du bitiment.

I. Chemin conduisant sous le pout F et sous le pont II, sous lecruel il y a une porte pour entrer sous le rocier sur leruel est le parillon $\mathrm{I}$.

F. Pont par lequel on arrive is la salle $G$ oi il y a une tente pour se garantir des grandes chaleurs. De cetce tente, en passant sur le pout $\mathrm{H}$, on cutre au petit parillon $\mathrm{I}$.

Fu sortant du bàtimcut on parvient à une salle eutourée de bancs de gazon, et bordće de trés-grands arbres. En continuant le chemin on arrive à la salle de repos K, au milicu de laquelle est placéc une volìre. Celte salle est entource de bancs de gazon cl, de trés-grands arbies à fleurs. $\Lambda$ droite en sorlant de celte salle, on arrive près d'une grille, de laquelle on apercoit la campanne.

\section{$\mathrm{N}^{\circ}$-9. JARDIY D AGREMENT.}

Ce jardin conticnt truis arpeus a deni.

A. Bàtiment ayant pour point de rue le temple de Minerve $G$, et le pont $C$ placé sur des roches sous lespquelles il y a des bancs. On a eu soin de placer en avant du pont une nasse darbres qui fait repoussoir. B. Pelouse plantée de moyens arbres, arbustes à fleurs et à odeur.

Partant du batiment, le chemin qui borde la prairie passe ì travers des massifs d'arbres toufus et des pelonses plantées d'arbres isolés, et conduit au pont C. Aprés avoir passé sous le pont, on traverse une prairie flantce d'arbres rares; lon monte insensiblement et l'on est surpris de passer sur un pont D. Cc pont est enfouré de très-grands arbres; on aperęoit sur la droite une salle de dause co gazon, désignée I, entourée de: bancs aussi cn gazon, et sur lit gauche se voit un massif qui sépare denx cliemins.

Cclui de droite est de niveau au-dessous du pont, trírerse une prairie, passe an pied du temple G. Le chemin de gauche, désignè $H$, monte frar une pente uı peu roide à travers des arbres très-touflus; ce qui fait opposition à l'ante chenin qui traserse la prairie.

En partant du pont D, on descend par une pente douce. Arrivé á l'angle des massils, on a pour point de vue le temple de Minerve G. En suirant le chemin on arrive à celui des maromiers, désigné E.

F. Chemin traversant les fleurs, les arbustes à fleurs, arbres à tiges et arbustes d'agriment, et de lá au temple de Minerve.

G. Tenuple de Minerve. En suivant le clımin toujours sur la droite, on passe sur le pont C et de láarrivé ¿ $\mathbf{K}$, on troure le chemin désinné $\mathbf{L}$, qui traverse le bois planté d'arbres plus élevés que les autres pour faire amphithèitre. Parvenu à la tète d'un massif $\mathrm{L}$, il y a me figure représentant Hyllus, un des fils d'Herrcule et de Déjanire. Le chemin de ganche conduit an pont $\mathrm{D}$; celui de droite à la salle de danse; de là aux remises $\mathrm{M}$, et ensuite à li basse-cour désignce $\mathbf{N}$.

O. Porte de sorlie.

\section{$\mathrm{N}^{\circ}$ 30. JARDIN CHAMPÉTRE.}

Ce jardin contient environ six arpens; il était presque de niveau, excepté à l'enchroit oú se trouve la source, près de laquelle on a établi le temple de Diane M, sur une éminence élevée de sept à huit pieds au-dessus du niveau de l'can. Les terres provenant des dẻblais du lac ont été tramsportées sur l'emplacemeut du teuple $F$.

A. Bitiment duquel on a pour point de vine le temple $\mathbf{F}$.

3. Pelouse plantèe d'arbres d'agrément. Sur la droite et sur la gauche, il y a des groupes d'arbres verts isolés, el sur le devant, des massifs de fleurs.

C. Lac an centre duguel se trouve lîle de Flore et le temple du mćme nom.

D. Chemin faisant le tour du jardin. Les nassifs bordant les murailles seront élevés par des terres provenant des déblais du lac, et ensuite plantés d'arbres dont le feuillage cacherait les murs. 
E. Chemin bordant li pelouse el conduisant it une sille de repos $\mathrm{S}$, entourée de grands arbres et de banes de gazon; de laquelle on a pour point de vue l'ile P, et le temple de Flore.

F. Temple d'Idmon, famenx devin, posè sur m rocher, sous lequel est une grotte dans laquelle on entre par le chenin $Q$, ct par la prairic, au point H. De cette grotte on a rue sur le bâtiment et sur la salle $\mathbf{K}$, au milieu de laquelle est la figare dllereule.

G. Chemin conduisant du bîtiment au temple F.

L. Figure représentant Diane, posée sur un tertre entouré de peupliers.

N. Figure représentant Hector. Vue de la salle $S$.

O. Salle de repos entource de peuplicrs et de bancs rustiques.

T. Pont hollandinis.

V. Pont chinois.

\section{$\mathrm{N}^{\circ}$ 3r. JARDIN D'AGRÉEENT.}

Ce jardin, contenant sept arpens environ, se trouve à mi-eôte. Les parlies de droite et de gauche sout très-ćlerèes.

A. Bâtiment ayant pour point de vue le lac B, au bout duquel se trouve un rocher, d'où l'eau tombe de roche en roche daus le lac. Sur ce rocher, ou a placé un temple grec $G$, entouré de saules pleureurs, peupliers d'Italic, d'Athenes, de tillculs, de ronces à fleurs doubles, cornouillers sanguins, des perventches, des iris, des rhododendrons, etc; sur la droite, on apercoit entre les arbres, le temple de l'Amour D; placé au ecntre de l'île d'Amour $\mathrm{C}$; et sur la gauche, la figure de Flore $\mathbf{F}$, posée à lia tète de lîle de Flore E. Sur la droitc du bâtiment $A$ se trouvent plusienrs têtes de massifs composés de très-grands arbres qui annoncent lentrée d’um grand bosquet, et sur la gauclıe, une prairie qui s’élère, et au haut de laquelle on a placé un obélisque $O$, cutouré de grands arbres.

Le premier dıemin qui se présente a droite du bátiment, suit le bord de l'ean et traverse une prairie parseméc de fleurs, darbustes à fleurs, de peupliers de la Caroline et de saules pleureurs. Arrivé í la tète d’un massif très-épais, le chemin de droite conduit tantồ dans un massif trés-touffu, tantôt à travers la prairie, plantie d'arbres isolés, différcns de ceux qui les précèdent et des massifs d'arbres, jetés sur la prairie. On aperçoit dans m lieu solitaire un hiosque $Q$, de lit l'on passe derriere le temple, d'oủ l'on a pour point de rue la caupagne qui se lie arec le jardin. En suivant le chemin, on arrive à une figuré qui représcnte Diane de Poiticrs. Arrivè à ume tête de massif, le chemin de droite forme le chemin de ceinture qui trarerse am bois très-touffu. Ce elemin monte assez rapidement et redescend de même. Arrivé au carrefour $\mathrm{L}$, on trouve un fontis assez profond. Le chemin de gauche conduit à la salle $K$, cutourée d'arbres, de bancs de gazon, et au centre plusieurs arbres isolés, tels que sipins pyramidals, etc. Suivant le clicmin de droite, on descend jusqu’à la tête du bois, pour rentrer à la maison du bicheron M, de laquelle on aperçoit la fontaine antique N. Ensuitr on descend par une pente douce à travers des arbres très-touflus et l'on arrive à une colonne antique $\mathrm{C}$. Le chemiu de droite descend par une peute donce eı va rejoindre le bâtiment $\mathrm{A}$; l'autre ehemin se trouve à mi-côte, et à une petite distance, on aperçoit, à travers une clairière, le tombeau $H$, arce un banc de gazon pres du monument et les saules pleureurs. En pareourant ce chemin, on arrive à une tète de massif; prenant sur la droite, on traverse les massifs d'arbres et la prairie plantće d'arbres isolès, qui se lient aree les arbres de l'ỉle de Flore. Parvenu un peu en avant du pont, s'offrent deux allées. Celle de droite conduit à une masse d'arbres très-toulfus, et cnsuite traverse une prairie complantée d'arbres isolés, avec des arbres rares et toujours variés; et de là au bâtiment : l'autre chemin conduit au pont près la figure de Flore $\mathbf{F}$; fait traverser lîle de Flore, plantée d’arbres à fleurs les plus rares. Après avoir passè un second pont, on suit le bord de la rivicre plantè d'arbres isolés et par masses autour du temple $G$, afin que le petit chemin qui monte ne soit pas aperçu du chemin de ronde. Arrivẻ au temple, on trouve une chute d'ear passant sous les pieds, et de là on descend à travers les roches, les saule; pleureurs, les peupliers de la Caroline : on traverse la prairie plantée d'arbres isolés d'espèces diflérentes. Arrivé au pont rustique, et de là dans líle d'Aınour, on trouve le temple $\mathbf{D}$, bâti sur une ćminence 
cutourée d'arbres et arbustes très-rares, tels que laalmia, azaléa, rhododendrum, et cufin tous les arbres qui viement dans ln terre de bruyère. Dans cette île, il y a deux chemins; l'un suit le bord du lac B , et lautre le hord de la riviere. Les arbres ct arbustes qui sont dans l'île se lient avec les arbres plantés dans la prairic. Aprés avoir parcouru les deux chennins, on traterse le pont, et l'on va rejoindre la tête lu massif rui est prè́s du bâtiment; on entre dans des taillis épais : le chemin de gauche fait passer dans unc prairie plantée d'arbres fruitiers. Ce chemin se trouve à mi-côte, ct va rejoindre la tète d'un massif: at très-pen de distance on voit un vase posé sur un piédestal, entourẻ d'arbres isolés. En détournant à droite, on trouve le chemin de ceinture qui est élevé, garni d'arbres très-touffus et vient rejoindre une salle de repos P entourée de bancs en gazon.

\section{No 32. JARDIN D AGREMENT.}

Ce jarchn contrent dis arpens environ.

A. Bàtiment ayant poü point de vue le temple d'Euryplule, désigné $\mathrm{R}$, le lac et l'ile $\mathrm{B}$.

Ce Temple est posé sur une éminence de cinq pieds, placée dans une prairie émaillée de fleurs, avee des arbustes et des arbres tiges à fleurs. $\boldsymbol{A}$ droite et à gauche du bâtiment $\boldsymbol{A}$ et à la tête du ruassif, qui ra rejoindre la salle, ete, on a planté des arbres dont la teinte est très-sombre, tels que nuaronniers d'Inde, ct attres, pour faire repoussoir; ce qui prolonge le point de vue sur le temple et la campagne.

C. Basse-cour. Le chemin de lroite du batiment traverse une partie d'arbres trés-touffus et eonduit rans l'île de Cythére B, en passant sur un pont gotlique, entouré d’arbres aquatiques. Les arbres de l’île sont des saules pleureurs, pcupliers d'Italie, peupliers d'Atlienes, peuplicrs beaumiers, blancs de Iollande, etc. Le chemin de droite de l'íle, cotorant la rivière, a pour point de vue l'autre bord du rivage. L'autre clıemin cotoye le lac et conduit au temple de Cythère désigné $\mathrm{D}$, posé sur un stilobate, ċlevé de trois pieds au-dessus du nivcau du terrain. De là on aperçoit, à travers de trés-grands arbres, le temple d'Euclée E, sur un rocher, et en avant un pont elinois. On a eu la précaution de laisser la rivière très-large en face du temple $\mathbf{E}$, les arbres assez ćloignés du rivage pour que ce temple se trouve trés-éclairé, ee qui fait contraste avec la riviére qui est rẻtrécie à une eertaine distance du temple, pour que les deux inonumens se trouvent dans le clair ct les eanx également limpides.

Pour sortir de lîle de Cythère, on passe sur un pont hollandais; on traverse une partie de bois et on arrive a une salle de verdure, entourée d'arbres. Sur la pelouse se trouvent des arbres jetés cà et là. On a eu soin de laisser des clairiéres, l'une sur la prairie, et l'autre sur le lac. En poursuivant sa route, on traverse tantôt la prairie, tantót des massiĺs, d'où on entend un bruit de clutes d'éan, on arrive au lieu d'où elles sortent. Le $\mathbf{t}$ mple $\mathrm{E}$ se trouye placé sur des roches, et là on voit l'eau tomber de sept pieris de hanteur. En suivant le même chemin, on arrive á une salle eirculaire entourée de bancs hollandais, et de là on aperçoit une rivière trés-large, dont les bords et les environs sont plantés de saules pleureurs, catalpas, tulipiers, sophora, etc.; ce lieu cst délicieux. Le chemin de gauche trés-touffu, parvient à un hermitage I, avec un petit jardin planté d'arbres à fleurs et une pelouse bordèe de toutes les espéces de roses, arec un bassin à lextrémité, et une salle de rejos L, entourée de bancs cliampêtres. De cet endroit, on aperroit le petit bassin et la prairie émaillée de fleurs; une autre salle $\mathbf{L}$ se trouve cntourée de grands arbres, et dans un endroit très-solitaire : ce lieu est destiné à la méditation. En sortant on traverse, à gauehe du bàtiment I, des massifs d'arbres, et on arrive à une salle de gazon destinće aux jeux, et de lá au bàtiment $A$ : mais quand on veut parcourir tout le jardin, on reprend à droite, à l'angle d'un chemin qui conduit à l'ile B. On trouve une route qui fait passer tantòt à travers les massifs, tantôt dans la prairie et sur le bord de la rivière; on arrive à cette salle dout il a été parlé, et passé cette salle, on se trouve sur un pont chinois, d'où l'on voit l'eau tomber du souterrain du rocher sur lequel est posé le temple $\mathrm{E}$, et la rivière qui serpente dans une prairie émaillèe de fleurs. Du pont, en suivant la même route, on passe devant un ha-ha; de là on aperçoit une campagne qui se lie avec le jardin; en continuant, une tête de massif se présente. Le chemin de gauche traverse de grands massifs et la prairie, et conduit à une salle entourée d'orangers, ete., et plantée d'arbres trés élevés sur le côté du nord; mais au eôté du midi, les arbres sont plus petits, pour 
ne pas priver les orangers du soleil : sorti de cette salle, on traverse les massifs, la prairie et le bord d. lac, d'oú l'on se rend au Jâtiment $\boldsymbol{\Lambda}$; mais si l'on prend le chemin de droite, étant au ha-lıa, ou travers: de grands massifs, et on arrive au temple d'Euryphile $R$, famense sybille de l’̂le de Samos. De lá on jouit pour point de vue d'ime prairie imaillée de fleurs et de l'aspect du battiment A. En suivant le chemin de ceinture, on parvient à une tète de massifs. Le chemin de gauche fait traverser une prairic plantée do massifs d'arbres et conduit au bàtiment $\mathbf{A}$, l'autre clımin fait arriver á une autre tếte de massif. le. chemin de gauche conduit au bátiment, et celui de droite á une salle de danse $T$, et salon de musique, avec des petits ehemins, lun desquels conduit à une salle où il y a une lualançoire $V$, et l'autre à une salle le repos près le bâtiment dans laquelle on a établi un jeu de boules.

\section{N ${ }^{\circ}$ 33. JARDIN DAGRÉMENT.}

Ce jardin contient onze arpens environ; il est bordé de deux côtés par une arenue de platanes.

A. Cour d'entrće.

B. Remises, écuries.

C. Bàtimeut principal, ayant quatre marches, pour descendre sur la terrasse.

D. Terrasse de laquelle on descend par neuf marches pour arriver au jardin.

Du bàtiment on a pour point de vue une prairie émaillée de fleurs. A l'extrémité est une rivière qui fait la fermeture du jardin, sur la façade du bátiment. Une rivière serpente dans la prairie, et vient se jeter dans un lae, désigné $\mathrm{F}$.

G. Ile de Nérće, dieu marin, fils de l'Océan et de Thétis. 11 épousa Doris, sa sour, dont il eut cinquante filles, appellées Néréides.

Cette île est plantée de peupliers blanes de IIollande, peupliers trembles, peupliers noirs, peupliers pyramidals, saules pleurcurs; à l'extrèmité une eabane de pêcheur.

E. Clemin traversant la prairie. Il suit le hord de la riviére, planté par fois d'arbres assez touflus pour nazquer la rivière, et tantòt assez clairs pour la retrouver; ses bords sont plantés de peuplier's du Canada, pet:plicrs liards et saules pleureurs, cte. De lá on arrive au pont romain, désigné $O$.

II. Chemin traversant de grands massifs d'arbres, et par fois des clairières, sur lesquelles il se trouve dics figures appuyces par des massils. On parvient de lá au pont turc. Ce pont se trouve entouré de peuplier s d'Athènes et autres; mais peu d'arbres, pour laisser apercevoir le temple d'Enesa $\mathrm{K}$, une des nymphes de la mer. On aperẹoit également le pont romain $O$. Un des côtés se trouve à l'entrée d'un massif, et váa rejoindre le chemin de ceinture, qui se trouve planté, à droite et à gauche, de peupliers, saules pleurears, saules marceaux, etc.; de là on arrive à une salle de danse, dẻsignée $\mathrm{P}$, plantéc d'arbres très - touffus, i $t$ entourée de bancs faits aree des trones d'arbres et des branches.

M. Pépinières.

N. Clımin faisant traverser le pont chinois J, qui se trouve placé au centre d'une rivière. Ce pont est entouré darbustes et de fleurs.

En cotoyant le lac sur la gauche, on entre dans un bois trés-épais. Un chemin conduit au pont de roches, $\mathrm{X}$ c lì on a pour point de vue la tour de Gabrielle T, placėe sur un tertıce, entouréc de frênes pleureurs, peuplicıss du Canada, tulipiers magnolias. En suivant le bord du lac, la partie de droite est plantée d'érables sicomores, ćrables de Virginie, érables à feuilles de frênẹ, des mérisiers à grappes, faux ébéniers, arbres de Judée, etc.; lilas, syringas, etc.; ct le côté du lac, de frênes à la manne, á une feuille, peupliers d'Italic, saules pleureurs. Ces arbres sont isolés sur la prairie et plantés par masses, fle là on ra rejoindre le bâtinnent.

R. Chemin conduisant au carrefour R. Le clemin de gauche traverse un massif très-touffu et va rejoindre le chemin de ceinture; cclui de droite fait passer une partie de bois très-touffu d'où l'or entre dans wne prairie plantée d'arbres verts, et où se trouve un tombeau antique, désigné $\mathrm{S}$.

V. Chemin traversant des massifs d'arbustes à fleurs et à odeur; il conduit à une salle de repos, avec vu bassin V, dans lequel il y a des poissons rouges. A la suite de cette salle se tranve une tête de massifs. İe chemin de droite traverse un grand massif llanté d'arbres de la seconde liauteur, pour se lier avec c: $4 x$ 
rle la route. On a eu soin de planter les masses d'arbres isolés sur la prairie, par groupes, tautòt rapprochẻs des bords des allées, et tantôt éloignés et disposés de manière à ce qu'on ne puisse pas aperceroir hiun chemin à un autre les personnes qui passent.

\section{No 34. JARDIN FANTASTIQUE.}

Ce jardin contient environ douze arpens

^. Cour.

B. Bàtiment ayant pour point de vue le lac C et l'íle d'Androgée P, fils de Minos. Des jeunes gens d'Athène el de Mérare le tuèrent par jalousie de ec qu'il remportait tonjours le prix daus les jeux. Sur la droite du bàtiment est une prairie énnilléc de fleurs et le lac $\mathbf{O}$, entouré d'arbrès isolés et arbustes ì fleurs. Sur la gauche on apercoit une pelouse plantée de massifs d’arbres fruitiers. Le chemin D traverse la prairie borlée de massils et d'arbres isolés; passe sur le pont égyptien $\mathrm{E}$, et eonduit au temple de Neptune F, posé sur un rocher, d'où l'eau tombe de roche en roche dans le lac C. Ce temple est entouré de grancles anasses d'arbres. En sortant du temple le chemin de droite traverse une pelouse plantée d'arbres fruitiers, et sur la droite, près de la muraille est une figure. Prenant à droite du point $G$, on suit le chemin rle ronde hordé de grandes masses d'arbres. Ce chemin monte toujour's jusqu'au temple de Jupiter $\mathbf{H}$, élevé de quarante pieds au-dessus du lac; de ce temple on apercoit le bàtiment B, entre les grauds arbres; en prenant le chemin de gauche du point $G$ on cotoye le bord du lac planté de peupliers, saules pleureurs, etc. Passant près le pout $\mathrm{S}$, on continue jusquau temple $\mathrm{H}$, dont il a été parlé. En sortant du temple, prenant le chemin de droite, on trarerse une prairie plantée darbres isolés et par masses; on arrive au pont chinois $K$, avec un petit hiosque, an milicu duquel on aperçoil la rivière serpentant dans le jardin et dins la canıpagne roisine; en eontinuant le elıemin on arrive ála salle de repos champêtre $\mathbf{L}$, dèrrière laguelle est une figure cntourée de grands arbres pour donner del'ombre. De cette salle on a vue sur la prairie en face, plantée de grandes masses darbres et des masses de fleurs, en face de la salle.

R. Chemin passant sur le pont ct traversant une île plantée d'arbres isolés; sur la droite est l'île d'Androgée P, à laquelle on arrive en traversant le pont rustique Q. Cette íle est plantée d'arbres rares afin de produire un beau coup d'ail du bâtiment. Le temple du même noun se trouve à l'extrémité de l'île, et entouré d'arbres isolés, et de fleurs sur ]e eôté du bàtiment $B$.

M. Chemm traversant la prairie plantée de masses d'arbres et d'arbres isolés. On arrive au bâtiment $\mathrm{N}$. Une partie servant de salle de bain et l'autre de gare pour les gondoles.

\section{$\mathrm{N}^{\circ}$ 35. JARDIN D'AGREIENT.}

Ce jardin contient environ treize arpens.

A. Bàtiment ayaut pour point de vue la prairie plantic de masses d'arbres à fleurs, sur le devant, tels que faux ébéniers, Sainte-Lucie, épines-roses, épines blanches, pêclıers à fleurs doubles, anandiers de Perse, lilas, syringats, pommiers à fleurs doubles odorant, toujours verts, rhododendrum ponticum, rosiers de toute espèce; plus le temple de Diane D, le temple d'Heetor E. Par-dessus le ha-la on apercoit la campagne qui se lie avee la prairie, ce qui auguente le point de rue.

B. Basse-cour, avec remises, écuries, toit à pores, etc.

C. Chemin traversant les massils d'arbres et la prairie plantée d'arbres isolés, conduisant au temple de Diane D, posé sur une éminence de dix pieds : le soubassement sert de glaciére; il est entouré d'acacias triacanthos, de bouleanx à feuilles de peupliers, ehàtaigniers, bouleaux, mérisiers, érables planes, etc. Sur le devant, des cèdres de Virginie, des bouleaux à canot et des arbustes variés. Le derrière du monument est l'entrée de la glacière avee une porte de sortie pour aller chercher la glaee. Le elıemin qui suit le bord de la nuraille est bordé d'arbres très-élerès et trés-touffus. Ce mènte chemin conduit à celui de ceinture, traverse de grands massifs et arrive à la lettre F. A droite est un ha-ha, par-dessus lequel on déeourre 
la campagne qui est très-variéc et qui semble faire partie du jardin. Le chemin qui est sur la gauche die temple D conduit à la tête du massif où se tronve un vase. En suivat le cliemin de gauxhe, on traverse la prairie, et l'on voit sur la droite, daus un bocage, une ligure T représentant Dirées, fille de l'Achéronet de la Nuit, entourie de peupliers et de fleurs. Cet endroit est garni de bancs champêtres; sorti de ce liev, on traverse la forêt et on arrive au temple d'Tfector E. Sur le derriére du temple sont plantés des lếtres, vernis du Japon, érables sycomores, et sur le devant des arbustes a fleurs, arbrisseaux. En tratersaut le taillis et la prairie on parvieut au temple d'Ilébé $\mathbf{G}$, et la se présente me très-belle frarie plantée d'arbres fruiticrs de toutes les espèces.

Le cliemin $\mathrm{F}$ traverse des grands massifs darbres variés el conduit it la chamièce II, dont le terranin est élevé de douze pieds au-dessus de celui du hâtiment, el de lí on jouil, frar-dessns lit untraille, de la vue la plus agréable el la plus éteudne possible. En suivant cette route, on arvive á un carrefour. Le dientin Mi conduit a travers le bois au fleuriste et a la serre chaude $\mathrm{R}$, arrivé à un triangle, un chemin conduit su: un pont. Par lautre clemin on parvient au bâtiment $\mathbf{A}$.

I. Chemin couduisant sur un pont rustique, placé sur des roches; du bàtiment on apercoit, par-rlessons

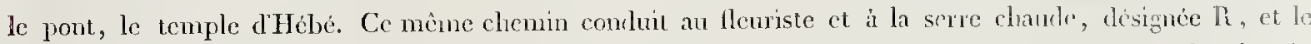
chemin $\mathrm{N}$ traverse le vignoble, les arbres fruticrs, et va au temple $\mathbf{G}$. Du temple, prenant le clicnin désigné $O$, on traverse la masse d'arbres fiuitiers et arbres à fleurs; onoarire au bîtimcnt $\Lambda_{\text {s }}$

S. Potager.

\section{No 36. JARDIN D'AGREMENT.}

Ce jardin contient treize arpens emviron.

A. Porle d'entrée. Le chemin qui est en face conduit au bàtiment désigné B, el les roitures tournent autour du batimeul el ront se rémuir à $\mathrm{C}$, où sont les remises, écuries, etc.

B. Bìtincnt ayant pour point de vue le lac désigné D, arec une île pllantéc d'arbustes à fleurs, comme althéa, rosiers de toutes les saisons, sécuridaca, chamoccrasus, trifolium, ctc, avec une ligure représentant Flore E. Plus une chule d'ean tombant de six pieds dans le petil lac G. licau sofl de ce lic a travers les roches et lombe dans un plus grand lac plus bas de douze pieds, ensuite passe sous le font I, posé sur des roches, et va alimenter les rivières qui serpentent dans la prairie, et former les lacs qui ont été creusés de trois pieł.ł. Les terres provenant. du déblais ont été jetées sur les îles. On a jlanté, pour retenior les terres, des plantar ds ou branches de saules, et derrière ces plantards, on a mis des fagots qui ont retcuu les terres. Les planiants ont poussés : il faut avoir soin de les couper tous les ans, et a l'estrémité des lacs ì la partie O; il conrient d'élablir une écluse pour l'écoulement des eaux, quand on veut retirer le poisson. Ces caux se reulent dians un lac que l'on a crensé de trois pieds plus bas que les autres; par ce moyen les eaux sont tonjours limi ides cl saines.

Toutes ces îles sont plantées d'arbres différens, tous arquatiques. On a en soin de placer les monumens dans les endroits les plus agréables.

F. Chemin conduisant au roclier au-dessus du lac $\mathbf{G}$.

H. Chemin conduisant au pont $\mathbf{I}$, et traversant de grands massifs d'arbres jusquau temple de Junon $\mathrm{K}$, duquel on apercoit une grande prairic plantée d'arbres.

L. Teinple de Viales, divinité qui présidait awx grands chemins.

I. Chemin conduisant du temple $\mathrm{L}$ au bàtiment $B$.

N. Chemin conduisant des remises à la porte A ct au temple L.

P. Temple de Faunus, tils de Picus, un des plus ancieus rois du Latium. De ce temple on a pour point de vue le Jaatiment $\mathbf{B}$.

Q. Pont chinois au milieu duquel est un hiosque, 


\section{$\mathrm{N}^{\circ}$ 37. JARDIN DU ROI.}

Ce jardin contient soixante-quinze arpens environ.

N. . . Rue du Jardin du Roi.

2. Riue de Buffon.

3. Boulevard de la Salpêtrière.

4. Esplanade du pont d'Austerlitz.

5. Quai Saint-Bernard.

6. Rue de Scine-Saint-Victor:

7. Jardin bas.

8. Première bande, cûté du midi.

9. Eutrée par la rue du Jardin du Roi.

10. Grande allèe de tilleuls.

г . Allée en terrasse sur li rue de Buffon.

x2. Petit bois, planté d'arbres de diverses espéces et de différens clinats, divisé par einq allées.

13. Cafǽ public et son jardirt.

14. Culture des fleurs annuclies, d'ornement.

15. Culture des plantes bisamuelles et vivaces de parterre.

16. Culure des semis d'arbres el arbustes de pleine-terre.

17. Latrines publiques.

18. Allie des tulipiers de Virginic. (Cette allée forme la lisne de démarcation entre l'ancien et le nouveau terrain qui composent le jardin.)

I9. Carré des arbres toujours verts, ou bosquet d'hiver.

20. Allée de méléze d'Europe.

2r. Bosquet d'autonme.

22. Allèe d'érables à fruit relu.

23. Bosquet d'etc.

24. Allée d'aylantlıes du Japon.

25. Bosquet du printemps, prenic̀re partie.

26. Deuxième bande, ou du milieu.

27. Esplanade d'entréc, par le pont d'Austerlitz.

2 . Culture des plantes médicinales, pour les paurres malades.

29. Carré d'étude de botanoplitles.

3o. Culture de fleurs des trois belles saisons.

3ュ. Bassin cufoncé pour les plantes aquatiques.

32. Quatre glacis, exposẻs aux quatre points cardinaux, pour la culture des arbustes.

33. Ligne alternative de kolrenteria, formant boule, et d'épines en parasol.

34. Pépinière d'arbres et arbustes indigènes et étrangers. Cćte partie est divisée en plusieurs earrés; pour les marcottes, les boutures, les greffes, les jeunes arbres, les grandes plantes, des planches de terrain de bruyère pour les rosages, d'autres pour les semis en terrines, et pour les repiquages d'arbres verts, ruchicr, lat rines.

35. Pépinières des plantes vivaces de pleine-tere.

36. Grand bassin pour les plantes aquatiques.

37. Cour des galeries de minéralogie et de zoologie.

38. Troisieme bande, ou du nord.

3g. Jardin de l'ancienne orangerie.

40. Bassin pour les arrosemens, et réserve pour les plantes nourelles qui fleurissent.

4r. Rampe qui conduit du jardin bas aux buttes; de chaque côté sont des glacis plantés d'ormille. 


\section{$(39)$}

42. École générale de Botanique; il y a quatre bassins pour les arrosemens.

L'étendue de l'licole de Botanique au Muséum dihistoire naturelle est de deux arpens deux tiers. Les plantes qu’elle renferme sont divisées en classes, fiuuilles, sections, genres et espéces. L Les classes sont au nombre de quinze, sans comprendre les genres incertains. Les fauilles, ou les ordres, forment un nombre de cent deux. Les genres composent un nomlure de quatorze cent vingt-huit á peu près. Les espèces sont en tout de sept unille deux cent somsante-luit. De plus il existe dans ce jardin un grand nombre d'arbres, d'arbustes ct de plantes vivaces qui, n'ayant pas cncore fructifié à Paris, ou ils sont inconnus, nont pu être détcrminés exactement, et placés dans l'École de Botanique, le nombre de ces đlerniers s'éléve à plusicurs centaines. Enfin les plantes printanières, automnales, les éphémères et les parasites, n’existant que pendant un temps trés-court, ne se trousent point dans la mène École, quoiquéelles existent dans différentes parties du jardin du Mluséum.

43. Allée de soplıoras du Japon.

44. École des arbres fruitiers de pleine-terre en France; il y a une cabane pour serrer les outils.

45. Allée de platanes du levant.

46. Ecole des plantes cmployées dans l'économie rurale et domestique des peuples de l'Europe.

47. Allée de catalpas de Virginic.

48. Ecole drs travanx, des préparations et des opérations d'agriculture.

49. Allée d'arbres de Judée.

5o. Bosquet de printemps, deuxième partie; ou y trouve aussi des échantillons des diverses terres, et des différens engrais.

5 r. Allée en terrasse, donnant sur la rivière.

52. Allée des marroniers, longeant la méuagerie.

53. Ancienne ménigerie des animaux carnacír's.

54. Fosses aux ours, sanglicrs, etc., etc.

55. Jardin de naturalisation dans lequel il y a un puit et un bassin.

56. Jardin des semis de végétaux de toutes les zones; châssis pour les végétaux de la zone torride; eoaches nues pour les semis des zones tempérées; chisssis en maconnerie pour la culture des plantes bulbeuses et tubéreuses: scrre à la hollandaise pour les jeunes plantes, des semis de végétaux du Cap, de l'Asie mineure, de la Nouvelle-Hollande; gradin à sept étages pour les plantes alpines; planches pour la culture des plantes uour elles; plate-bande pour les plantes des climats froids.

57. Petite butte, en amphithéâtre, plantéc d'arbres verts.

58. Jardin de lamphithéâtre.

5y. Jardin de professeurs, administrateurs.

6o. Grande butte, vulgairenent nommé Labyrinthe, et plantée d'arbres verts.

6r. Cèdre du Liban, planté par M. de Jussieu, en 1736.

62. Kiosque portant un cadran solaire.

63. Chalais.

64. Alléc de pins, dominant la rue du Jardin du Roi.

65. Massif de thuyas de la Chine.

66. Jardins de professeurs, administrateurs.

67. Cour de l'amphithéâtre.

68. Cabinet de botanique, laboratoire de zoologie et salle d'arlministration.

69. Par'cs des animaux herbivores de la méuagerie, vulgairement nomunés vallée suisse.

7o. - des moutons d'Egypte.

7 r. - des mérinos ct moutons du Cap.

72. - des mouflons, bouquetins, gazelles, biches indiennes.

73. - des buffles et des oiseaux aquatiques.

74. - des dromadaires, des cazoars et d'autres oiseaux.

75 . - des autruches et des cigognes.

76. - des jeunes faisans et autres gallinacées. 
7. Pare des cléphans, bisons el antres.

78. - des ricbres.

79. - des bones et cherres.

80. - des daims blancs, noirs et ordinaires.

8r. - les axis.

82. - des cerfs clu Gauge, et de dains.

83. - des moutons de differentes races.

24. - des cerfs et de lichies de direrses variciés.

85. - de l'algazelle.

66. - nourcat.

87. Supplúment de l'École des arbres fruitiers.

88. T'crain nouxclicunest acquis.

89. Chemin de ronde estéricur de la mènagerie.

90. 1'ropriété étrangère an Nusćun.

\section{RENYOIS ALX FABRIQUES FIGURÉS EN ÉLÉTTION.}

9r. Grande serre tempéréce, de trente-trois toises de long; la partic de gauche du bátiment renferme les logennens de jardiniers, les ateliers de treillageurs, charrous et autres; deviere se trouve une pompe mue par des chameanx, et qui fournit l'cau à la scrre et à la ménagerie.

92. Nouvelle ménagerie des animaux fỉroces.

93. Facade des galeries d'histoire naturelle.

94. Calbane des cerfs du Gange, et autres.

95. Amplitheitre pour les cours publics.

96. Retraite des buffles, cu au-dessus des bouquetins.

97. Falmirue it quatre parillons en crois, avee colombicr.

98: Chammiere des zidures.

99. - des moutons mérinos et du Cap.

roo. - russe pour les chamois, bouquctins, etc.

ror. - des autiuches el cazoars.

102. - arabe pour les chamcaux.

ro3. - pour les boues.

104. Farake de la grille d'entrée, par le pont d'Austerlitz:

105. Pavillous du portier.

ro6. Corps-de-garde.

107. Ancicnne orangeric. Au-dessus d’clle, derrière une terrasse, se troure la lanterne des cierges du Péron, et les serres des plantes de la zone torride.

ro8. Scrte voùce.

109. Serre froide, el au-rlessus serres de plantes grasses ou suceulentes.

I ro. Serre des arbrisseaux des tropiques et des plantes alpines.

r I. Serre des outils de l'École de Botanique.

r 2. Serre des plantes de la zone torride, ou serre Buffon.

113. Vue en perspective de la grande butte et du hiosque qui la temine.

i 4. Tue en perspective de la petite Julle.

1 5. Chaumicre oì se font les cmballiges des jecunes plantes, ct oì se trourent les dépôts de terre.

I 6 . Cabane des daims noirs.

I 7 . - de l'algazelle.

I 8 . Café public.

I19. Cabane propre an nouveau parc, $n^{\circ} .86$.

120. Grande rotonde servant d'écurie à l'éléplıant et autıes herbivores des pays chands. 


\section{( II $\left.^{\mathrm{I}}\right)$ \\ RETTOIS AUI BATIENS FIGUBZS SUR LF PLIN.}

I21. Maisen de professeur el du farte les galeries.

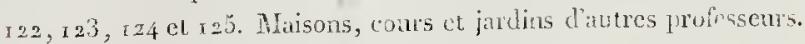

126. Cour el ćcuries.

127. Cour dite de la Régie. Le bátiment de droite est le Cabinel d'Anatonie comparće; le batiment de gauche, sert de logement à des aikles-naturalistes et aux employés du Muséum.

128, r 29 et 130. Maison et jardin d'un professeur, atelier de serruic et autıes.

I3 1 . Hénagerie des singes et voliere des oiseaux des pays clauds.

132. Voliere pour les oiseaux de proic.

$133 \mathrm{et}$ I 34 . Maison particulicre.

Ce jardin, ainsi que les batimens et fabriques, ont été exécutés d'aprés les planes et sous la clizction de IT. le clucralier Nolinos, insjecteur-général des traraux publics de Paris, arelitecte cle la ville, ete.

\section{$N^{\circ}$ 38. JIRDIN D'AGREMENT.}

Ce jardin coutient vingt arpeus curiron.

A. Porte d'entréc, avec deux parillons destiućs pour le portier.

B. Chemin bordé de trés-grands arbres, conruisant au bâtinent C.

C. Bàtiment placé sur une éminence, entouré de pelouses émaillées d'arbustes à fleurs et fletros, ayant pour point de rue, du côté de D, une prairie qui se lie arce la campagne, ainsi que du côté de S; du côté de la pe-

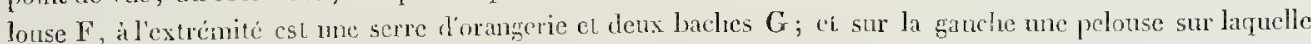
sont établis le temple de la Gloire T, el une voliere $\mathrm{U}$. Ces objets sont placés sur des éminences, et les prairies sur lesquelles sont établis les montuncus ont été creusécs: les terres nut été placécs sur les massifs d’arbres el arbusies, ec sui dome au terrain de lia variété el de l'étrudue.

S. Chemin traversant les massifs de flcurs et d'ableres; il conduit an temple de la Gloire 'T', an-derant duguel on a placé des arbies verts pour faire repoussoir, et autour du temple, des arbres à tiges et arbustes i fleurs. En suivant ce mème liemin, on arrive it uie figure ll aulour de laquelle se tronvent des banes chamfútres cutourés darbres plus élerés que les précélens. Sorti de ect entroil, on traverse une prairie plantée d'arbres isolés arec des massifs placés sur la pelouse, qui empêchent dlaperceroir tonte l'étculue de la práirie, rarie la promenade et conduit an temple de Cérès Q. Le clienin P' trarerse une grante partis de pelouse plantéc de unsses darbers isolés, et conduit an bàtiment $\mathbf{C}$.

K. Chemin lraversant un bocage frès touflu, conłluisant à une salle de repos designéc L, entomée de luustes représeutant les frands lommes : celte salle est élevée de quatre pieds. Passé cette salle, on se troure dans un carrefour; le chemin de droite fait arriver à une pelouse coucare, désignéc $O$. Deux figures placées atx deux extrémités seraient entoures diarbres va riés, avec des banes clampètres, etc

N. Chemin conduisant aux chàssis ot serres chaudes. Ce même chemin conduit an chemin de ceinture, qui, eu le suivant, fait traverser un taillis trés-épais, et aprés aroir passé par le temple Q, concluil á une salle irrégulièrefdésignéc $\mathbf{I}$, cntourée de banes el chaises. Arrivé derrière le temple de la Gloire T, il y a denx chemins; l'un est le ehemin de ccinture qui passe derant le ha-ha et conduit à une salle dans laquelle il y a $u$ groupe de liğtres désigné $\mathrm{V}$. En sorlant il y a deux clıemins, l'un contuit au bátiment, et l'autre passe devant le ha-ha, ct conduit à la porte d'entréc ct de la aux remises et écuries $H$

\section{$\mathrm{N}^{\circ}$ 39. JARDIN DAGRÉMENT.}

Ce jardin conlicut vingt-un arpens environ.

A. Porte d'entréc, avec le logement du porticr et du jardinier.

B. Bàtiment ayant pour point de rue une inmense prairie, plantée sur le devant d'arbres verts, et le temple de la Concorde E, placé sur une éminence de einq pieds, entouré de veruis du Japon; sur la gauche du 


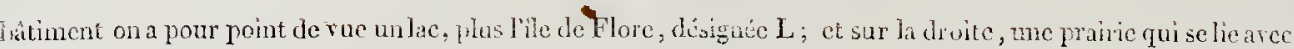

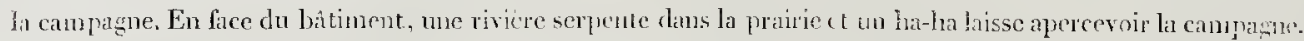

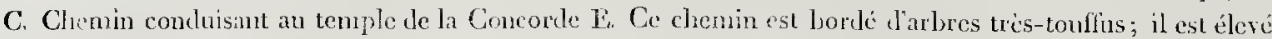
le quatre pieds, jnsquau deuxiome C, et descend par une pente douce jusquiau ha-ha qui se trouve sur la druite, de lá on jouit d'un point de vue très-aşréable; sur la ganche lu la-ha se troure une prairie renfoncée, et les arbres d'alentour sont placés sur me cuninence, ce rui rend cet endroit solitairc. On a formé hes bancs de gazon irréguliers. Sorti de celicu, on arrive à un angle de massif; sur la gauche on apercoit une figure représentant Epigies, nymplie de la Terre, posée sur mu tertre, sur lequel il y a des arbres isolés entourćs te banes champêtres. On tronre a la títe du bois, deux chemins; celni de droite est tantít élevé et éloigné

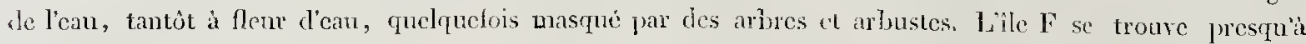
lextrémité de lin riviére; elle est plantie de sunles pleureurs el peupliers, par stonpes : on a placé sur cette île le temple de Plutus, dieu des ricliesses. On traverse un bois tris-touffu, et on arrive a un pont chinois C, qui, de lì, laisse aperecroir le plus beatu point de vue quilil soit possible de voir; sur la troite, deux rivieres vicment se precipiler dans un lac. A la naissance de ces dicus rivièes, on troute le temple de Mars $G$, contouré de penpliers, blanes re Jollande, saules pleurenrs, saules ordinaires, trembles, etc; sur la gancle se voit un immense lac, clans lequel eśt me île an milieu de larpuclle est placé le temple de Consus II, dien du conscil. Cette île est plantíe drartres aquatiques, par groujes. Une autre petite île se troure isolée dans le lac.

Passé le pont on apercoit une téte de massif. Le ehemin de gauche traverse la pranie, suit le bord lu lac. Avant diariver à une autre tîte de massif, on traverse un bois trics-tonffu qui cache le lac : une trés-grande prearie se tronve sur la droite aree des arlores isolés, el des massils darbres et arbustes, qui cunpèchent que l'on n’apercoive le clımin de roude qui est plus élevé de huit pieds au-dessus de celui qui borde la rivière. Le che-

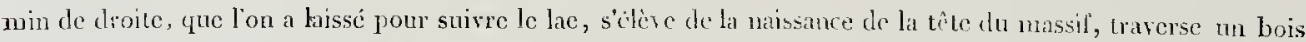
trés-touffu, el va rejoindre le chemin de ceinture qui est devé de lauit picels, conduit à un pont gothique qui se trouve à l'extrémité lu lac. De li première tête de massif, on trouve le chemin de droite qui lait arriver á

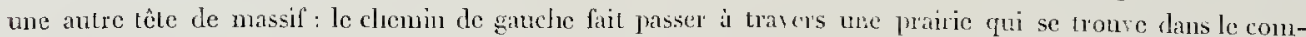

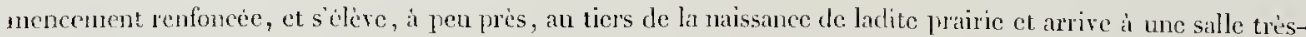
́́leréc; de là, á travers les arbres, on aperçoit la campagne et une partie du jardin. Cette salle, désignće I, cst entourée de banes el de fişures; í l'extrénité de la salle est un chemin gini descend et ra rejoindre le chemin de ceinture, dont nous avons parlé plus haut. Arrivéà une tête de massif le elemin de gauche conduit au pont gothique, dont nous arons parlé; ct le chemin de droite, gui est le clicmin de ceinture, trarerse une pranie, plantée, sur la droite, d’urbres isolés; de l'autre cóté, les nuassifs d’abres et anbustes jetés par groupe. Ce mène chemin trarerse une prairie plantée d'urbres par masses, á travers lesquelś on aperêil une petite île cntourée d"arbres aqquatiques, el au centre une fis̆ure représentant Euplurosine, l'une des trois Grices. Passé cette prairic, on trouve un chemin qui traverse le bois, fiit passer devant la lasse-cour désignée M. Le chemin de gauche conduit sur un pont hollandais, de la au bîtiment. L'antre chemin fait passer devant la porte dentre designec $A$, passe sur un pont rustique : de ce pont on apercrit le beitiment et la campagne; puis on arrive à un trianłle planté d'arbres et arbustes. Le chemin de droite est celui de ccinture; il naverse la prairie, et l'aulre conluit at bitiment B. Le ehemin de gauche du bâtiment traverse deux massilis d'arbres, cotoye la riviere, et conduit daus l'ile de Flore, désignée I., plantée d'arbres tiges, arbustes et arbrisscaux à fleurs, avec une figurc représentant l'lore, deesse des fleurs. Sorti de cette île, on suit le bord de la rivicie; on arrive à un massif : le elienin de droite fait nonter à travers un bois trés-ćpais, á une salle de repos, désignée $\mathbf{K}$, entourée de bans champétres; an centre de celle salle est une figure représentant Diane, déesse des lorc̀ts. On aperçut à travers les arbres une très-belle rue, comme ćtant élevée de vingst pieds au-ricssus du niveat du bâtiment. Sorti de cette salle, on traverse le bois et on arrive à l'ile II. En sortant de cette île, le chemin de gauche conduit au pont chuois $\mathrm{C}$, el arant d'y arriver on a un!s dhemin sur Ja choite qui traverse le bois trés-épais et la prairie plantée de massils d'arbres isolés. Prenant le chennin de droite sortant de l'ile, on traverse une praire plantée d’arbres isolés : arrivé à l'anghle d’un massif, le chenin te droite suit le bord du lae a fleur d'can, et va rejoind'e le pont gothique : le clicmin de gauche, qui est à mi-cóte, arrive à un carrefour planté d'arbres trés-touffus a ce chemin conthic à la sille de Dime $\mathrm{K}$; et celui de droite conduit au pont gothique, tandis que le chemin qui est en face fail descendue dans un rallon planté diarbres ariés, suit le bord de la rivière et condut au batiment. 


\section{( 43$)$}

\section{$\mathrm{N}^{\circ}$, 0 , JARDIN D'AGREMENT.}

Ce jardin contient quarante arpens cnviron.

$\Lambda$. Porte d'entréc et logenent du portier.

B. Bátiment placé sur me ćminence et ducquel on apercoit une prairie, an bont de laruelle est le lac D.

Sur la droite du bàtiment est un chemin qui conduit au temple de Mars, désigné $\mathrm{F}$. Arant dly arriset on traverse dcux masses d'arbustes á fleurs. Arrive à nue tête de massif on trouve sur la droite nu chenin qui traverse la prairie, fait arriver à un pont chinois et an chemin de ceinture. Du pont on apereit ums rivière qui serpente dans la prairic, tantòt masquée par des arbres et tantôt aperợe : ce qui excite l'iutícit ; car il ne faut pas tout roir d'un seul coup d'ocil. Passé le pont on artive au temple de Mars F, placé sur nue éminence entourèe d'arbres, arbustes et quelques arbres isolés pour varier la scéne. De ce temple ou aperçoit la rivièe daus une plus grande étendue, qui est tantòt large, tantôt plus ćtroite. Ia partie la plus large est proclie le monument. It faut planter le moins d'arbres possible, afin que l'ean paraisse liminide. Passé le pont, en suivant les nurailles bien masquées d'arlures, on traverse les massif́s. Arrivé á une tìte de massif le chemu de droite est celui de ronde, qui quelquefois traverse la prairie, les masses d"arbres, et arrive à différens points de vue sur la caupanne; fait passer sur un pont rustique. Arrixé sur ce pont on découvre tur très-bean lae, et au centre nue île $K$ plantéc d'arbres et arbustes : la prairie qu'on apercoit est plantće de groupes d'arbres opposés les uns anx autres. Parvenn à me tête de massif, un clicmin conduit an temple grec G, qui n’est apereu que de bitiment; celui de droite traverse la prairie alin qu'il ne soit pas apereu du bâtiment, dont il est très-près, il a été crensé d'un pied; traverse un bois très-ćpais ct arrive ì um angle de massif: le chemin de gauehe conduit au bitiment, et celui de droite traverse me prairie pantée d'arbues isolés. $\Lambda$ ganche, passé les arbres isolés, on apercoit le bútinent et sur la droite la campagne rui se lic avec le jardin. Dés quon a passé le bàtiment on cutre dans le bois : on arriłe à différens chemins; liun va à la porte

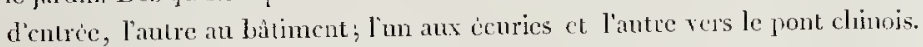

De la droite du bâtiment, à lopposé de la lettre $\mathrm{R}$, le chemin de gauche traverse la prairie, près la rivière; il traverse des massifs, entre dans le bois et à la tête du premier massil : le cliemin de ganche laisse apercevoir nue prairie plantée d’arbres isolés, par masses; unc figure représentmul l'Initiè est posée sur une ćminence entource de peupliers. En suivant le chenun qui conduit au bâtimesıt, sur la ganche, cst nu obèlisque cntouré de masses d'arbres. Cet obèhisque est anssi sur nne éminence, et aperẹ dı batiment : en suivant le cliemin on arrive au bûtiment.

S. T’allée qui est sur la gauche de bâtiment trarerse le bois. $\Lambda$ partir de la tète du massif, le chemin qui est

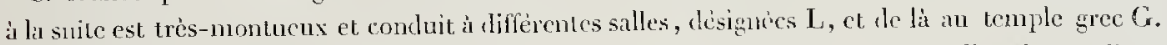

Du bâtinent on aperçoit différentes allèes : les unes font monter ì différeutes salles élevies. D'autres chomins traversent la prairic, la forèt et vont au pont hollandais I. Reprenant le chemin qui borde le lac sur la gauche, on arrive à une tête de massif. Le chemin de droile monte à une salle designée P, élevée de quinze pieds an-dessus du lac, entourée de bancs et de figures. De cette salle, cutre les arbres, on décourre la campagne, le báliment et le temple de l'Amour K, placé dans lỉle de ce nom. Cette île est liée à une autre î̉e par uu pont, et un autre pont conduit au chemin de ceisture.

\section{Nं 4. JARDIN D'AGRÉMENT.}

Ce jardin contient ringl-six arpens enriron.

A. Porte gothique.

13. Grange.

C. Pressoir tare.

D. Colomlier gothirque.

E. Four. 
F. Ecuries et remist:

G. Busse-conir.

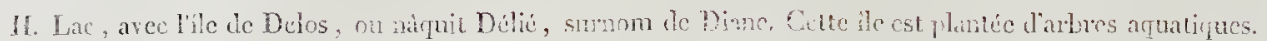

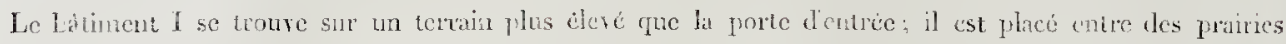

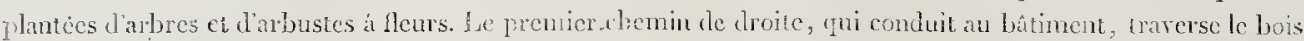

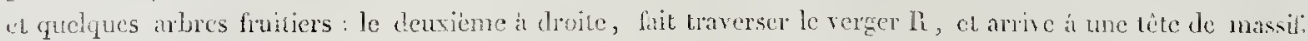

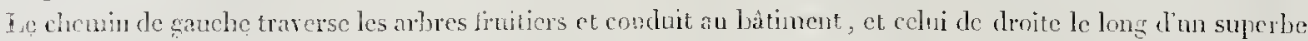
espalicr. Arrivé à un ha-ha, le chemin de gauche est borlé d’abres dagrénent, sur la pelonse, afun de

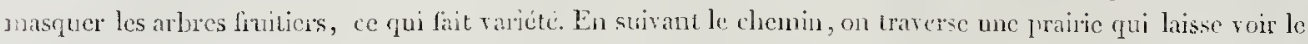
Jatiment, sur la ganche el sur l. droite on aperroit la campagne par-dessus les ha-ha, Parenu á une tête de

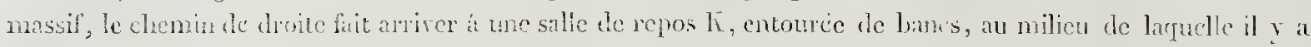

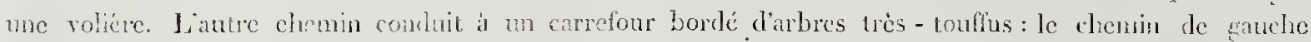

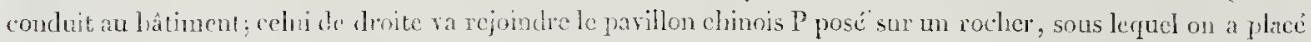
des glaces qui réptent la campagne el le jerchin. Des cscalicrs tont monter à travers les roches, au parillon chimois 1"; de lit on jouit de la flus belle vue possible. Revennt au chemiu du carrefour, celui gni conduit at

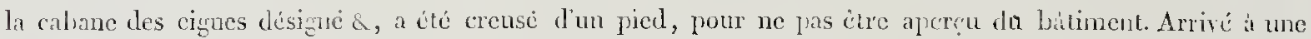
lète de massif, le chenju de gauche fait traverser sur lil droite un massil trés-épais; de l'antre côté, ce sont des

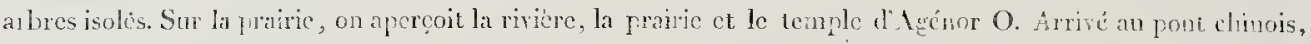
placé sur des roches, on a sur la droite, pour point de rlie, le lac L, cutouré de san!es pleurenrs, peupliers dTalie, sanles ordinares, cle. Arant damiver an jont, est un themin qui conluit á un carrelour. De ce carrefour on rencontre sur ha gauche le chemin qui conduit au pont. Celui de drofte ra ane rober P; celui qui

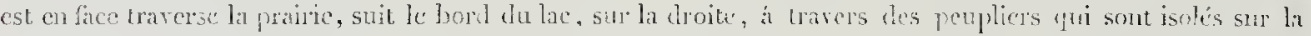

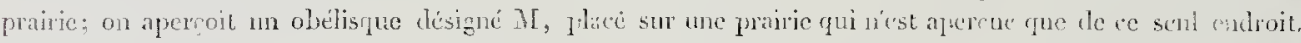

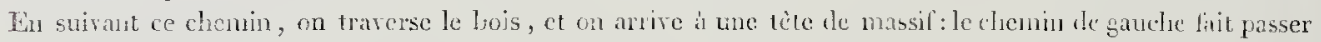

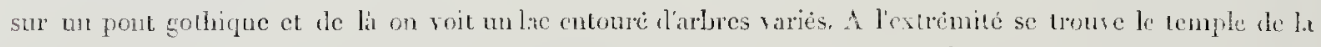
Concorde $F$, josé sur un melier : lwois sources sortent du dessous du temple, pon alimenter les lacs. Le

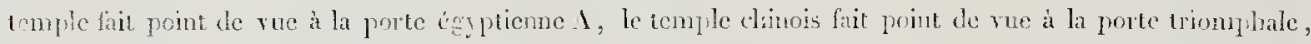

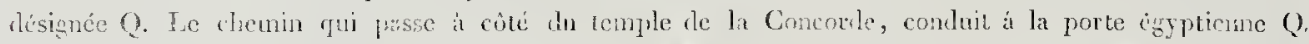

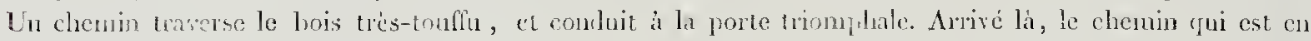
fire traxcrse un bois immense, puris conduit á différeus chenins : l'un passe au-dessus du second lac, fait prasser

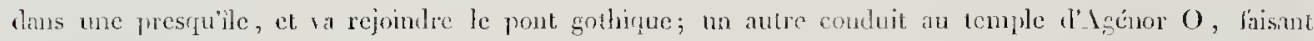
point de vue au chàtean, nn autre sur le pont clinois. D'autres tratersent différens massifs d'albres, On revient au chìtcau. Du chàteau, le chemin de gauche lait passer sur un pont én roches, ayant sur la droite

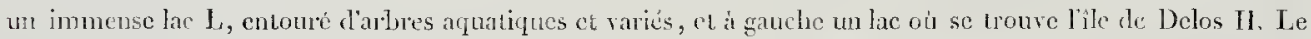
chenin de gauche lint iraverser un bois tres-sombre. Arvivé is un triangle, au centre, on trouve uu tombean $\mathrm{X}$. En suivant le clocuin, on trarerse la fortet la prairie. Partem a une tite de massif: on détonne át ganche, on traterse un bois, et on arrive a un ha-hn qui laissc voir la campagne. Du chateau on passe devant la basse-conr G. Le chemin qui est en lace, comuluit a travers des arbres isolés et plautés par groupes. On suit le bord d'un lac qui conduit au dâtiment. En poursuivant te chemin, on passe devant le colombier gothique et le pont ture. A partir des renises, le chenum est borké d'arlores fruitiers, et les murailles qui sont au nord sont bordécs l'albres d'agrément qui se lient áce ceux qui laissent aperceroir le bâtiment.

V. Polager.

S. Orangerie.

\section{$\mathrm{N}^{\circ}$ 42. JARDIN FANTASTIQUE.}

Ce jardin conticnt deux cents arpens euviron.

A. Porte d'entréc avec leux pavillons.

B. Batiment en colonnades, ayut en face le lac $\mathbf{K}$, a gaudie une prairic qui so lie arce lis campagne, á droite on a pour point de vuc un lac, une prairie plancic darbles par masses; à l'estrémité un ha-ha. 


\section{(45)}

C. Pont c'n roches entoure de peupliers ditalie et de saúles plenrenrs. Passé ce pront on trouve une

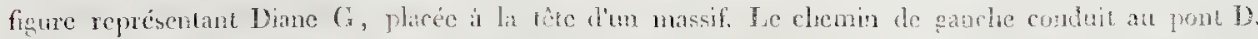

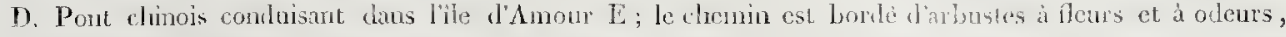
et sur les bords de l'cen des soplomas, des fulipiers, catalpas, des peupliers d'itatie.

F. Temple de l'Anour ayant pour point de vue le temple H. Passé l'ile on traverse un pont lonllandais et on arrire à une tête de massif. Le chemin de gatuche fint traverser la prairie plantée d'arbres at fleurs. Par leschairieres on a des points de vue sur la scande ile $\mathrm{N}$, flantée d'arbres isolés et par masses, de maibre que, diune alle a une aute, on ne saperenve pas, ce qui agrandit infuiment le trrain. Il faut aroir soin de placer, dans les endroits les plus agrèales, des bancs ou des canapés. Fin suivant liz pronemade on arriwe au tenple des IIoraces II, posé sur un rocher sons lequel est la décharge d'ean. Le clemin de droite contuit directement, en trarersant la pranie ct les uussifs d’arbres, au temple de Cérés désigné \&, entouré darires a fruits. Le cheniu de droite est le hemin de ronde qui traterse de grands nussils, On arrive an temple de Diane Y, posé str une éminence de six picels, et faisant point de vue au bâtiment. Arrivé à G est une fignre representant Dine. Le chemin de droite conduit sur le pont gothique $\mathrm{R}$; de lí on voit la riscibe qui serpente dans la prairie plantée d'irbres aquatiques varies. Enstite on traverse un bois très-épais oin se troure nue salle de repos avee des banes rustiques, et de lí on arrive au bâliment. Le chemin de gauche traverse le bois, le pont rustirue $\mathbf{S}$, et suit le bord de la riviére. Arrice al la grille dientrie $\mathbf{A}$ on a poum point de vue le temple de Nincrve désigné $\lambda$, posè sur un tertre tés-édevé at entouré darbres verts. Une tête de massil forme l'enbranchement de deux chenins. Colui de droite suit le bond de la rivière, fait traverser la salle de repos; de lí on arrive au cluateau.

Liantre clıemin traverse un-bois tres-épais et conduit au bitiment. Du bitiment le clıenia de gauche conduil à une tète de massif. A gauche est me salle pittoresque avec des Jancs. Le chemin qui est en arant de la salle, rst hordé diabres et arbustes á fleurs, trarerse la pritie. On a eu soin de creuser le cliemin ponr que l'eau du lac se lic avec la prairie. De lí on ari ive au pont désigné $\mathrm{O}$, duquel on a pour point de vue nne inmense prairie traversée par me riviére qui serpente. Les arbres plantís antour du pont font avaut-sciene pour le temple des Ioraces II. La se troure un chemiu traversant la prairie, le pont hollandais, entreconpe de saules pleureurs el de peupliers dithenes : il conduit au temple II. Le chemin de gancho suit la numille, bien bordéc darbres pour la nasquer, et apres aroir parcouru me partic du bois on trome une clainiére qui laisse apercevoir le pont désigné $O$. C'est sous la masse de peupliers qui borde la rivière quion doit placer un hane pour jonir de cette vuc. En suivant le chemin on arrive à nu autre Tranc, at la téte d'un massif désigné $\mathrm{L}$, qui a pour point de vue, a travers une pririe, le temple designá $\mathrm{N}$. Enfin on arrive à me tête de massif. Le chemin de gauche conduit à la salle cle repos et de lá ant bàtinent. Liantre chemin fail traverser la prairie, le bois, et revient it la grille deutrée. On passe sme le pont $S$, do lit aux remises et ćcuries $\mathbf{T}$, desquelles on apercoit l'obélisque $\mathrm{Q}$, placé au milicu diune prairie. plantée d'arlres et arbustes variès.

U. Orangerie.

V. Hangar destiné pour les poteries et les ustensiles aratoires.

X. Potager.

Y. Lac.

\section{$\mathrm{N}^{\circ} 4^{3}$ JARDIN D'AGRÉLEYT.}

Ce jardin contient environ ringt-luit arpens.

Le båliment $\mathbf{C}$ se trouve sur un terrain très-élevé. On a pour point de vue le temple de Mars, désigné $\mathbf{T}$, élevé de dix pieds an-dessus du niveau du lac; en face du hâtiment une très-forte masse d'arbres divise une grande prairie, et snr la gauche on apercoit une prairie avee un ha-ha qui lie la cam. pagne avee le jardin; à droite du bâtiment on voit un lae qui se trouve an bas du vallon; un ha-ha par-dessus lequel on apercoit la campagne voisine, et le temple grec $H$ cntouré d'arbres isolés.

A. Porte d'entrée arec deux parillons et un laa-ha de clargue cótć. 


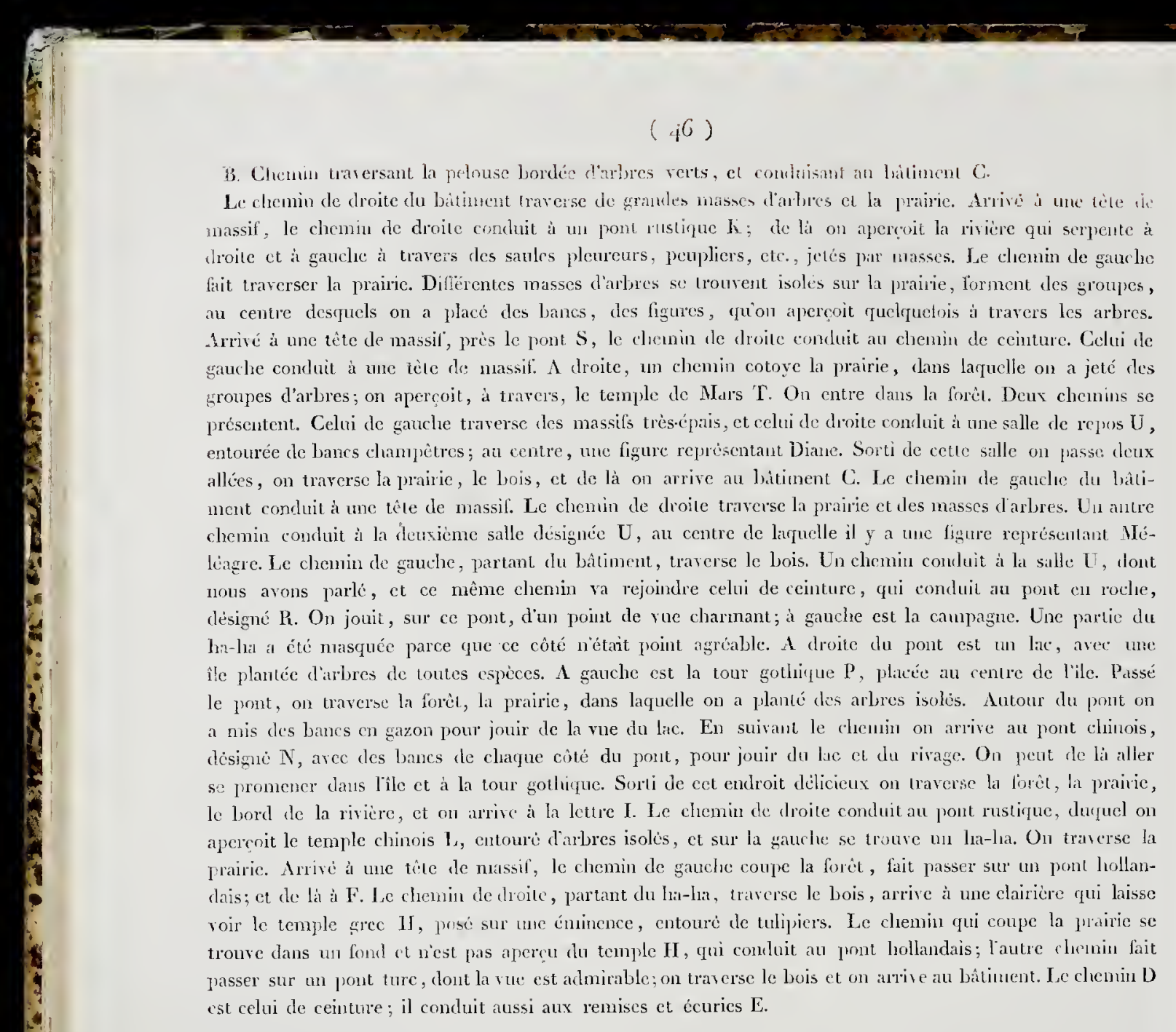

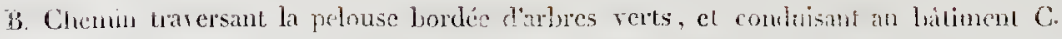

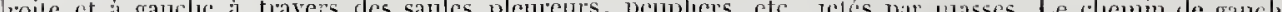
ait traverser la praire. Dillerentes masses d'arbres se tronvent isoles sur la prairie, forment des groupes,

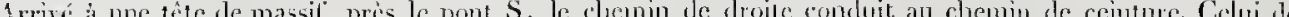
sauche conduit à une ièle drs nuassif. $A$ droite, un chemin cotoye la prairie, dans laquelie on a jeté des troupes d'arbres; on apercoit, a travers, le temple de Mars $\mathbf{r}$. On entre dans la forét. Deur chemins se présentent. Celui de gauche traverse des massifs très-épais, et celui de dinite conrluit à une salle de repos U, entourée de bancs clanupêtres; ai centre, une figure représentant Diane. Sorti de cette salle on passe deux allćes, on traverse la prairie, le bois, et de là on arrive au bitiment $C$. Le chemin de gauclue du bâtiment conrluit à une têle de massif. Le chenin de droile traverse la prairie et des masses diarbres. Un antre chemin conduit à la deuxième salle désignéc $\mathbf{U}$, au centre de larquelle il y a unc figure représentant Hékiagre. Le chenin de sauche, partant du bấtiment, traverse le bois. Un chemin concluit à la sille U, dont uous avons parlé, et ce même chemin va rejoindre celui de ceinture, qui conduit au pont chr roche, désigné R. On jouit, sur ce pont, d'un point de vae charmant; a sanche est la campagne. Une partie du hi-lia a été nasquée parce que ce côté nètait point agréable. A droite du pont est un lac, avec une île plantée diarbres de toutes espéces. $\boldsymbol{A}$ sauche est la tour gothique P, plicéc au centre de lile. Passe le pont, on traverse la foret, la prairic, dans laquelle on a planté des arlores isoles Autour du pont on a mis des bancs en gazon pour jouir de la vue du lac En suiraut le cheulin on arive au pont chinois,

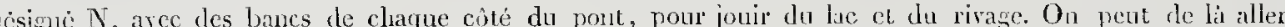
so promener. daus lîle et a la tour sothique Sorli de cet endroit délicieux on traverse la lorè la pranic,

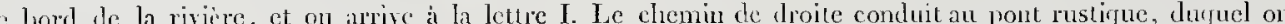
prabic Aprive a une tîle de nussif, le chemin de gauche coune la foret fait passer sur un pont hollandais; et de lá à F. Le chemin de droite, partant du ha-hat, trawerse le bois, arrive à une clairière qui laisse voir le temple arec IJ, prose sur une énnence, entouré de tulipiers. Le cliemin qui coupe la pranic se trouve dans un fond it nest pas apereu du temple II, qui contuit an jont hollandais; lautre chemin fait est celui de ceinttite; il conduit aussi aux remises et écuries E.

\section{$\mathrm{N}^{\circ}$ i i. JARDIN D'AGREMENT.}

Ce jardin contient trente-deux arpens en iron.

A. Porle d'entrèe avec denx pravillons.

B. Clımiu conduisant au bátiment C. Ce bàliment se trouve dans un vallon. Une pente douce en gazon, ornée de fletirs près le bảtiment, est arrètée par un immense lac, avec différentes rivières qui serpentent dans plusieurs prairies bordćes d'arbres précieux, et par masses. On roit plusieurs autres groupes d'arbres, arbusles et arbrisseaux. Au centre du lac U est lỉle de Nérée T, dicu marin, plantée d'arlures aquatiques et variés; et au centre de l'ile le temple du mème nom. Denx issues font traverser le lac pour aller daus cette île par le moyen d'un va-qui-vient; l'une des issues est près de la salle désignée I; l'autre prés du temple chinois $\mathrm{N}$. Ce temple est aperçu à travers les arbres qui sont cn avant et placé sur une éminence, de manière à dominer sur le lac, et former point de vue pour la salle I. A droite du bàtimeut est une prairie plantée d'arbres, les uns isolés, d’autres par groupes, arec des arbrisseaux ct arbustes a fleurs. Au centre des massifs se trouvent des arbres forestier's; à l'estrémité de la prairie un ha-ha qui laisse voir la campagıe qui se lie avec le jardin. Sur la gauclıc du bâtiment est une prairic qui śélève assez rapidement, et it lextrémité un pièlestal sur leqnel il y a un groupe de figures.

D. Différens cliemins. Liun traverse la prairie, la pelouse, et va rejoindre le pont elinois $\mathbf{E}$, sur lequel 
Il y a une petite fahrique, lien de repos. De la on aperçoit plusicurs rivières qui serpentent dans la pratrie, prarfois plantíe d'arbres isolés.

Un autre chenin passe à trivers la forcht, lis prairie, et sur fo hord de la ricierce. Un autre traverse Inn loras de rivière par le moyen d'un pont rustique, fait arec des branches d'arbres; il suit le bord du lae, el ra rejoindre le temple chinois $\mathrm{N}$. Un chemin, qui n’est pas aperçu dn tomple, passe derrière à travers de trés-grands arbres. Arriné à une certaine distance du chenin on apercoit une partic de prairie (qui se trouve sur lis gatuche, au centre de larguclle il y a une figure représentant Diane. Des bancs de gazon sont placés cà et lid. Ce bosquet est un licu trantquille et solitaire. Sorti de cet cullouit, on passe dans le bois, et on va retrouver le petit chemin du bord de leau; de lis on arrise it un pont holfandias, d'oin on aperçoit un proint de vue charmant. Sur la gauche, lïle de Neréc T; sur la droite nne rivière qui serpente à travers lis forêt, la prairie, et passe sous le pout chinois E. Te chenin du pont E a $F$ traverse la forêt et la prairie, et va rejoindre, en montant, Je temple de la Victoire I. De lá on a la plus belle rue possible. Sur la gauche du temple on aperceit différentes rivières qui serpentent dans une immense prairie plantèe de masses d’arhres de mème nature, mais diffèrens pour chaque masse. Sur la droite, une prairic qui se lie avec la campagne par le moyen d'un ha-ha. Ondescend une petite montagneà travers les érables, et on arrive ì un carrefour. Le chemin de gauche fait traverser une prairie plantée d'arbres isolés, et conduit dans un hosquet d'artres très-touflus, ou se trouve le temple de Junon désigné $G$. Ce temple est aperrn a travers les arbres, et fait point de vie du temple de la Virtoire f. En suivant le cliemin de la prairic c1s parvient an pout hollandais, qu'on laisse sur la gauche: on suit le bord du lae, et ou arrive à un pont en roche. Du pont, d'oụ l'on jouit du plus bean point de vne ruïil soit possible de voir, on apercoit sur lit Iroitc differentes rivières qui se précipitent de roche en roche dans différens lacs à lopposé. Dilléreus chemins se présentent. Celui de gauche conduit à lia lettre II, oi 'st un obélisque placé à la tète d'un massif. Le ehemin de ganche conduit à la salie I et an bâtiment; l'autre chemin aboutit à plusieurs autres frui traversent la forêt. Tantiot on rencontre des clairières au travers desquelles on aperçoit difiérentes figures. An centre de la furcit se troure une salle en gazon désignée $O$, au centre de laquelle se voient des figures posécs sur des piètestanx, arec des bancs faits de branches d'arbres.

Q. Chemin conduisant à une salle de jeux ou de repos $\mathrm{R}$, de lì à un pout ture $\mathrm{S}$; il traverse la forèt, passe devant le ha-ha, puis derrière le temple $G$, el arrive à une têle de massit. Le chemin de ganche conduit au front en roclee, dont nous avons parlé. Le chemin de droite devient un pru montueux , traverse les pins, sapins, les roches, et enlin arrive au temple de Neptune M, posé sur un rocher : les colonnes de ce temple soit aussi en roche. L'ean sort à travers les rochers, se prescipite de roche en roclie, de lac en lac, et va se jeter dans un immense lac U. On tronve diffërens chemins: qneliques-uns traversent les roches. Les rivièrer, dont les eaux tombent de cascade en cascade, ainsi que les autres, sont bordées d'arbres verts et de roches. Dans quelques parties de prairic, on a placé des tombeaux, des pyramides V. Ces objets sont entourés de massifs d'arbres variés. Les cliemins de gauche du bâtiment passent à droite de la basse-cour P. Il en est un à ni-côte qui va jusqu ì la rencontre d’une tête de massif. Un des chemins de gauche est triesrapide, et passe it travers les pins, sapins, rochers: il conduit au temple de Neptune; un antre, également à mi-côte, va rejoindre le pont en roclıe, de manière que du bàtiment on aperçoit un amplhithèatre qui luit un poiut de vie agréalle et varić aree le côlć opposé.

\section{$\mathrm{N}^{\circ} 4^{\bar{j}}$. JARDIN D'AGRÉMENT.}

Ce jardin contient environ trente-quatre arpens.

A. Porte d'entrée à laquelie on arrive par une avenue de platanes; devant la porte est un ehemin eonduisant au bâtiment C, placé á mi-côte; la prairie de droite monte insensiblenent jusqu'au temple de Saturne F', (fils du Ciel et de la Terre). Il est cutouré de massifs darbres variés; tels que micocouliers, charmes, alisiers, muriers à papier, cerisiers ì flcurs doubles, Sainte-Lucie, aralics épineux, lilas, syriugats, cornouillcrs sangaius, elc. Du bâtiment on aperçoit trois autres prairies variées par leur forme, et plantées d'arbres 


\section{$(48)$}

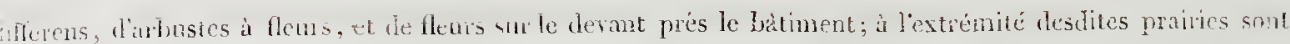
les ha-lia par-dessus lesquels on aperecoit lit compane qui se lie avec le jardin.

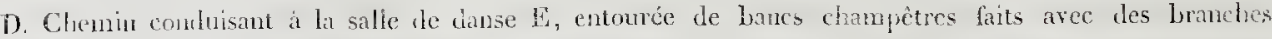
l'aubres; derricre ces banes sont plantes des epines roses, - il flenrs blinches, - it fleurs donbles, libs de

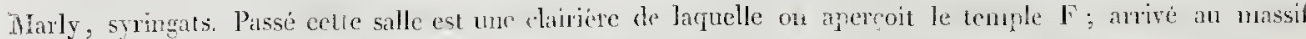
formé en triangle à la lette $U$, il y a ì chaque angie une figure; liune représentant Diane, l'autre Méléagre, "t la troisiène Jamus.

G. Le clemin de droite conduit à la statue dEuterpe, une des nenf Muses, désignée II. Cette tigure pat placéc dans un vallon un pen protiond; ce qui fait opposition arec le temple F, qui est sur un monticule;

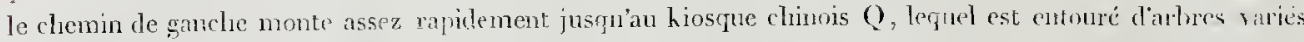
r.t vus du betiment. A travers les branches d'arbres on découre la campagne, qui est vingt pinds autirssous du nivean du hiosqne (); en suivant le mime cisemin on descend inserusiblement au carrelonr wit est placé un obchisque. Le chemin de droiie va au chemin de ronde; on passe prés une dairicre, de lanuctle on apercoit un vallon trés-protond drrièe le hiosqne Q, dont les talus sont plantés de chénes, de hères, etc.

P. Chemin qui traverse le bois ct conduil ì une tète de massif; le cliemin de droite travre nne prairic plantie d'arbres isolés; tels que pins du Jord Weimouth, pins d'Ecosse, ete.; ch contimunt on arrive à une fignue 'T (représentant Crané, nymple, l’une des femmes de Janus), placée à lamgle du massif. Le chemin de gautche,

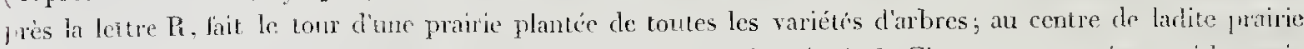
est le temple d'Jtar jalices $\$$, la plus belle fille d'Argos. Elle fut fort aimce de Clymenus, son picre, qui la maria aver beateup de peine; ct aussitút qu'clle fut mariée il fit mourir son gendre pour la répentre, cte

U. Chemiir de ronde.

V. Pavillon chinois placé sur une éminence chtonrée le grands massifs d'arbres, et sur la prailie des arleres isolés: tels que bonducs, bouleaux, micocouliers, ete. A ganche de cette pratric est ure salle de renos, entource de bancs el quelques rases : cet endroit est un lieu dr méditation.

I. Verger de toutes les especes darlires frubiers.

h. Potager au milicu des quarrés oú sont des bassins pour les arrosemens.

1. Serre chroule.

M. Baches pour les ananas et plantes étrangèes.

N. Châssis pour les melons.

O. Remise, écurie, bassc-conr.

\section{$\mathrm{Y}^{\circ}{ }_{46}$. JARDIN D'AGRGMENT.}

Ce farlin contient cinquante-rleux arpens emiron.

A. Arenue d'érubles panachées, au bont de liquelle est une grille et deux pavillons.

13. Chemin conduisant au pont chinois F, surmonté d'un kiosque. Sur la ganche on apercoit un immense lac, à croite l'île de Calypso $G$

C. Bâtiment placé ì mi-còte. La laçade du côté du temple dithloménéc, désigné $\mathbf{N}$, a pour point de vue une raste prairie plantée sur le devant d’arbres verts. Sur la droite de la prairie est une salle trés-élevée servant de salle de danse, clésignée I. A droite du bàtiment se trouve lỉle de Calypso $G$, avec une fignture représentant $A$ lceste, fils d'A gamenuon et de Priseïs. Sur la gauche du bàtiment une prairie, et à l'extrémité un ha-la qui lie la campagne avec le jardin. En arant du bàtiment est un lac bordé d'arbres aquatiques, et à l'extrénité un ha- ha.

D. Remises et ćcuries.

E. Lac entouré d’arbres, tels que saules pleureurs, peupliers d'Athènes, suisses, etc.

Le chemin de gauche fraverse la forết, va au pont sur lequel il y a un hiosque, et rejoint le chemin de ronde. Ce chemin est très-fourré. Le denxième chemin traverse la prairie plantée d’arbres isolés, et suit parfois la rivière. Le troisième chemin conduit sur un pont rustique dans l'île désignéc $\mathrm{G}$.

Le chemin de roncle $i \mathrm{i}$ traverse une partic de la forèt, suit parfois la rivierc, passe sur un pont ture, 
1raverse la forêt, arrive á une balançoire $\mathrm{P}$ qui se trouve entourée d'arlres d'agrément, en sursant une tête de massif qui se trouve sur la gauche. Le chemin de gauche traverse me partie de bois, ct á une certaine

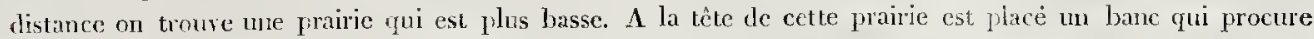
un point de vue chamant. Ce mène clıemin conduit au temple $\mathrm{N}$. De là une tête de massif se présente. Le chemin de droite fait passer à travers le bois et la prairic plantée d'arbres fruiticrs; on arrive an temple de P'omone $\mathbf{K}$, faisant point de vue au bâtiment principal. En suivant la même route on traverse le bois, la prairie, et on arrive a mne salle $Q$, dans laquelle il y a un grand banc; et de lì au bâliment, cu traversaut une forct tres-épaisse. Le chemin de droite de la balancoire P traverse la forêt ct conduit $\dot{a}$ la maison de bûcheron $\mathrm{O}$, d’oú on aperçoit le temple d'Eole $\mathrm{L}$, dicu des vents, posé sur un tertre trèsélevé; en suivant le chemin de ceinture, on arrive a $S$. De liz on jouit, i droite, de la vne sur la campagne; et à gauclıe, de celle du bâtiment. Arrivé à me tête de massil, plusieur's clımins se présentent. Le premier à gauche traverse la forêt avec rapilité, et fait pisser devant et rerrière le temple d'Eole, entouré d'arbres verts rares : ces clicmins se rencontrent et conduisent au bàtiment.

Le denxieme cliemin de gauclıe traverse une prairie plantée d’arlures isolés, et vient rejoindre un chemiı qui suit le bord de la riviére, passe devant le lac, et de là au bâtiment. Le troisiéme chemin traverse le lois, passe sur un pont fait en roche $\mathbf{R}$, suit la prairic, tantôt prés de la rivière, tantôt il s’en éloignne. Arrivè à une tète de massif, le chemin de ganche conduit au bâtiment, et l'autre à la grille l'entréc. Deux issues conduisent à la basse-cour D.

\section{$\mathrm{N}^{\circ}$ 47. J ARDIN D'AGRÉMENT.}

Ce jardin conticnt environ soisante arpens.

$\Lambda$. Porte d'entròc.

13. Remises, écuries et ferme.

C. Pont clinois.

D. Logement du proprictaire, ayant pour point de vue plusieurs prairits, aver des masses d'arlures en arant des chemins pour les masquer de manie it ne voir que prairies, abres, eaux ct fibriques, et a cinpêcher que les rivieres ne puissent étre aperenes d'un seul coup d'ail.

Sur la droite du bàtiment on aperçoit, à travers les arbres, une salle de danse $\downarrow$, posée sur une éninence, et une prairie plantée de très-grands arbres. Sur la gauche une prairie traversée par une petite allèe, unis qui n’est pas apercue, ayant eu soin de creuser cette allíe. Du côté de la rivière on récouvre un immense lac $\mathrm{X}$ avec des îles et des ilots.

E. Chemin traversant la forèt et la prairic. Arrivé à unc tète de massif, on trouve deux chemins. Celui de gauche est de niveau avec le bâtiment; il traverse la prairie et le bois, longe la rivière et ra rejoindre le pont égyptien F.

G. Chemin un peu montueux, conduisant à la salle $\mathbf{H}$ où se trouve une volière, entourée de bancs. Sorti de cette salle, le chemin de droite fait passer sur le pont rustique I, composé de trones d'arbres, et de li dans l'île d'Amour désignée K. Cette íle est plantece de saules pleureurs, peupliers d'Italie, de frène à la manne, à une feuille, et d'arbustes à fleurs, etc.

Le chemin de droite a la lettre $\mathbf{G}$ traverse la forc̀t, la prairie, le bord de l'ean, et condnit au pont turc U. Ce pont a pour point de vue une rivière qui serpente dims la forêt, et d'oú l'on apercoit l'ìle d'Anour K. Passant sur ce pont, on trarcrse la forèt et on arrive au pont égyptien F; de lá on jouit d'un très-beau point de ruc. Sur la droite, on a ve sur la campagne; et sur la ganche, la rivière qui serpente dans la prairie plantée d'arbres isolés. Suivant le chemin de ronde, on traverse la forèt et la prairie : on arrive à une tète de massif. Le chemin de gauche fait arriver à une salle pittoresque entourée de bancs turcs, avec un obélisque M qu'ou aperçoit du bâtiment à travers les arb’es, et de lă au bâtiment. L'autre chemin suit la muraille, qui est masquéc par des arbres et arbustes bien fournis de branches, comme des épines, sureaux, ete, et on arrive à liłle d'Idalie désignée $\mathbf{R}$, dans laquelle se trouve le temple de Mars désigné $S$, qui a pour point de vue la campagne, les rivières et une partie da lac. 
Deux rliemins se presentent : celui de ganclıe fait trarerser cleux ponts. Le premier est un pont rustique ęui lie l'ile de Pandore avec l'ile d'Idalie Q; l'autre est un pont lollandais, et de lá on arrive au bâtiment. Ces denx îles sont plantées d’arbres variés.

Lautre chemin fait passer sur un pont gotliqque, traverse la forêt, la prairie plantée d'arbres isolés, suit parfois le bord de la riviére et quelquelois s'en éloigne, ee qui rarie la promenarle. Arrivé á une tête de nuassif, un chemin conduit au pont clinois, et l'autre suit le clemin de ronde, passe derrière la ferme. En traversant la forèt, on arrire à un tombeau antique, placé dàns un lien ombragé par de grands arbres, autour duquel on placerait des bancs rustiques. Sorti de cet cnçroit mystérieux, on traverse la forêt et la prairie, et on arrive au temple de Diane désigné $\mathrm{Y}$, placé dans une prairie, appuyé par des masses de vernis du Japon, des hêtres, etc. En snivant le chemin, on parvient au pont égy ptien désigné F. En sortant du bâtiment, prenant le chemin $\mathbf{D}$, on nonte par une pente douce au sommet du labyrinthe $\mathbf{O}$, sur leruel est placé un cabinet d'astronomie. De ce labyrinthe on découvre toutes les fabriques du jardin et la campagne; le chernin de la montague sera planté de toutes lcs espèces d'arbres rerts.

\section{$N^{\circ}$ 48. JARDIN D'AGREMENT FANTASTIQUE.}

Ce jardin contient environ soixante-dix arpens.

A. Cillle d'entrie á laquclle on arrive par une arenue de tilleuls.

B. Cour:

Du bàtiment $\mathrm{C}$, on a pour point de rue une prairie émaillće de fleurs, plantée de très-grands arbres; au contre des massils et sur le devant, des arbres demi-tiges à fleurs et arbustes à fleurs.

D. Chemin traversant le bois : Ics bords de la prairie sont plantés de groupes d'arbres, et sur le bor du lac se trouvent des arbres isolís. Arrivé ì une tête de massil's, le chemin de droite traversc le bois ct arrive au temple gotluique de Comus désigué $\mathbf{G}$, dont les fonctions étaient de présiler aux réjouissances nocturnes, aux toilettes des femmes et des jeunes gens qui amaient la parture. Ce temple se troure placé á mi-côte et cutouré de très-grands arbres; à droite et à gauclıe deux masses cncadrent le bátiment, de manière qu'on apercoit une trés-belle prairie et le pont désigné $\mathrm{F}$.

II. Chemin assez rapicle passant derrière le temple $G$, traversant le bois très-épais ct revenant au temple d'Erginus E, roi d'Orchomène. Il fut en guerre avee Hercule, qui le vainquit, le tua et pilla ses Etats. Ce temple est entouré de trois massifs; il a sur sa droite une prairie escarpée plantée d’arbres isolés ; sur sà gauche une autre prairie plantée d'une très-forte masse d'arbres qui masquent le temple désigné U. (Sur le jlan, ce temple en élévation est marqué de la lettre $V$ ).

I. Chemin traversant la forêt et la prairie plantée d'arbres isolés. Arrivé à nne tête de nassif, le chemin de gauche traverse l’ile de Médon K, un de ceux qui voulurent épouser P'énélope pendant l'absence d'Ulysse; ce fut aussi le nom d'un Ccntaure, etc. Cette île est plantée de saules pleureurs et autres arbres aquatiques. L'autre chcmin traverse un bois touffu, et arrive à une clairière qui laisse apercevoir la rivière et l'ìle $\mathbf{K}$. En contimuant le chenin, l'on rencontre sur la prairic des arbres variés, plantés isolément, cutre lesquels on aperçoit une prairie plantée de grandes masses d'arbres pour ne pas aperceroir d'un seul coup d'cil toute l'étcnłluc du terraiı. En suivant le bord de l'eau, on arrive à un momment gothique L, plaré sur une éminené entourée darbres isolés, duquel on aperçoit un petit lac, une prairie, et me rivière qui scrpente á travers les arbres. Au côté de droite est une prairie sur laquelle il y a deux figures, l'une représentant Ericthée, classeur que Minerve prit soin d'élever, et l'autre Ericthonius, fils de Vulcain. Il fut roi d'Athènes. Arrivè au pont hollandais désigué $F$, on a sur la gauche une large prairie; sur la droite des rivières, des ponts, des arbres plantés antour des rivières: et en suivant le chenin, on troure sur la droite une inmense rivière; sur la gauche une prairic plantée de très-grands arbres; l'on arrive a un pont rustique qui fait passer dans l’ile de Calypso; au centre de ceuc ille est le temple de Calypso, fille du Jour, désinné Y. Du pont hollandais on parvient à une tête de massif; sur la droite est une prairie élevée, sur laquelle on a placé unc colonne $Y$. Sur la gauclıe se trouve le temple d'Hamadiales U, nymphe des bois, fille de 
Nirce et de Doris. Ce temple est posi sur une émincnce, faisant point de vne au bâtiment principal. Dirfèrens cheunins traversent la forèt et plusicurs prairies sur lesquelles on a placé des ligures, des rombeaux, etc.

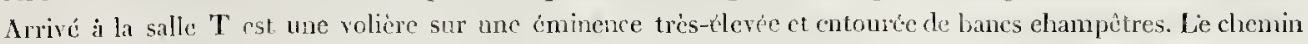
désigné M traverse le bois, eonduit à une salle de danse, avec des bancs de gazon, ct au centre de cette sále csi une figure désignée $\mathrm{N}$; de cette salle on aperçoit, à travers les inasses d'arbres, une maison hollandaise désignèe $O$, sur un tertre ayant différens points de vue. En suivant le chemin on traverse la forêt et la pelouse sur liquclle on a jeté des masses d'arbres. Arivé à $\mathbf{M}$, le chemin de gauche conduit au bâtiment $\mathrm{L}_{4}$; lautre chenin traversc le bois. $\Lambda$ unc eertaine distance on apercoit une prairic plantece diarbres isolis, et lentrce d'une riviére dans le parr. En suivant le bord de l'cau on arrive à une tète de massif. Le chemin de droite fail passer sur le pont dessigné P, ct l'autre conduit au pont gothique F. P'assé le pont P, on traverse me prairie plantée d’arbres variés; et sur la droite du clıemin, on trouve des ha-la qui laissent aperceroir la campague qui se lie avec le jardiı. Sur la gauche on ap̣erçoit la rivièce, tantôt masçuée par des nasses d'arbres isolćs, et d'antres massils bien épais. En continuant le chemin, on passe daus un bois toulfu, et a l'extrémité on arrive à un pont. rustique lait avec des troncs darbres. Sur la gauche du pont on a pour point de vue une rivière qui serpente dans la forèt, et à l'extrémité la tête de l'île $\mathbf{Y}$, sur laquelle se troure une figure reprèsentant Hercule. Sur la droite, on a la rivière qui sort dı jardin et coule à travers des pâturages oi paissent des troupeaux de moutons. En suivant le chemin de droite, on trouve des arbustes, des arbrisseaux et arbres qui doivent cacher la muraille; à gauche sont des clairicres qui laissent apercevoir sur la prairie, des pins et des sapins sur la partic haute, et dans le bas de la prairie, des peupliers, baumiers, ete. On arrive a $R$, au temple Danaxandra, héroïne révérée comme une décsse dans la Laconie. De ce temple désigné $\mathbf{R}$, on aperçoil la eampagne, la prairic, etc., et de là on parrièt au château.

\section{Nं $49 \cdot$ JARDIN CHAMPÉTRE.}

Ce jardin contient cnviron quarante-quatre arpens. (Il faut doubler l'échelle quai est sun le plan).

A. Porte d'entrée gothique, avec deux pavillons pour le portier.

13. Prair ie plintée d'arbres et arbustes à fleurs.

C. Bâtiment du proprietaire placé sur une éminence de sept pieds au-dessus du niveau de la porte d'enirée, et de l'autre còté faisant face au jardin qui est de nireau jusqu’à l'ile G. A l'extrènité du terrain, à la lettre \& il $y$ a trente pieds de profondeur, de nanière que le bàtinent a une trés-belic vue.

D. Cour des remises, écuries. Tेo $^{3}$. Abreuvoir entourć d'arbres.

E. Potager avec quatre bassins pour l'arrosement des liggunes, avec uue serre d'orangerie, des châssis.

F. Chenin conduisant à travers le bois et la prairic. Arrivé á ıme tête de massif, le chemin re gauche conduit à l'íle d'Amour désignèe $G$, avec le temple de ce nom. Cette île est plantè de toutes les espèces de rosiers, et fleurs de toutes les saisons. Le premier pont que l'on a traversé est un pont hollanilais entouré de saules pleureur's, peuplier's d'Italie et autres. Ie deuxiéme pont, qui est à la sortie de l'ille, esí un pont chinois H. Le temple qui se trouve á l'extrémité de l'île a le plus beau point de vue possible, portant sur une partie du jardin et sur la campagne. On entend le murmure des eaux qui tombent du rocher qui se trouve un peu plus loin que la tête de l'ile. Arrivè au pont anglais qui fait passer sur la riviére, on a pour point de wue, à droite du pont, la chute d'eau dout on a entendu le murmure, et à gauche deux autres chutes d'eau. En suivant le clemin on traverse une prairie, une portion de bois, et on arrive au temple de Cérès K, fille de Saturne et de Cybèle, déesse de l'agriculture. Sur la droite de ce temple, se trouvent des céréales avec quelques arbres fruitiers, et sur la gauche une prairie qui, par une pente douce, va border un lac entouré d'arbres isolés entre lesquels on aperçoit à travers la prairie et la eampagne. Sur la droite du temple le chemirı conduit à une tête de massif; le chemin de droite conduit à une salle de repos $\mathrm{I}$ entourèc de bancs champètres; cette salle est très-solitaire. L'autre chemin fait traverser la prairie, les céréales, le verger désigué sur le plan V. A gauche du temple de Cérès se trouve un chemin qui traverse de très-beaux arbres, et ra rejoindre le sommet du roeher de Neptune $O$. On a planté entre 


\section{(52)}

ces roclies des ronces à fleurs doubles, des jasmins, des ehèvrefeuilles, aristoloches, cornouillers sanguins. Le chemin cle droite traverse la forêt, et va rejoindre le clıemin de ronde. $\boldsymbol{A}$ gauche, on descend à travers Jes rochres, les pius, les sapins, ete., et l'on arrive au chemin qui borde le lac. Un petit seutier fait arriver sous le rocher sous lequel est placée sur des roches la statue de Neptune. A droite et à gauche, sont des bancs en mousse et pierres, sur lesquels on respire le frais; de la on a, pour point de vuc, un lac bordé d'arbres variés. Sorti de eet endroit charmant, reprenant le chemin qui borde le lac, on arrive au pont gothique; de là, clans l'ile de l'Amitić, désignée N. Cette île est plantée d'arbres les plus rares, venant dans la terre de brnyère. On a eu soin de placer un banc entouré de fleurs, en face du pont ehinois MI, posé sur des roches. Au-dessus du pont plusieurs clutes d'ean tombent dans un lac. Sorti de ce lieu agréable, on passe sur le pont rustique. Le cheınin de gauche du pont conduit et fait passer sur le pont $\mathrm{M}$, et l'autre chemin conduit au elıemin de ceinture. Sur la droite du bâtiment C, est un chemin qui conduit à une salle de repos où il y a unc volière, et sur la gauelı̀ du bâtliment un chemin qui descend par une pente douce, et fait arriver à eelui de F.

R. Chemin montant et traversant les arbres fruitiers et le vignoble. On arrive au temple de Bacchus $\mathbf{S}$, fils de Jupiter et de Semclé. Arrivé à unc tête de massif d'arbres fruiliers, le chemin de gauche conduit au chemin de ronde $\mathrm{X}$, et eclui de droite à un bàtiment hollandais $\mathrm{V}$.

T. Chemin creux traversant le vignoble, et allant au bàtiment $\mathrm{V}$.

X. Chemin de ronde traversant la forêt, une partie de prairie, et conduisant au bâtiment gothique Y, serrant de logenıent aux gardes. En suivant le ehemin de ronde \&, on traverse la prairic, la forêt, et l'on arrive sur la gauche à une tête de massif qui laisse apercevoir deux ehemins. Celui de gauehe se trouve être de nivean, et va á l'ile de l'A mitié; l'autre monte assez rapiclement au pont chinois $\mathrm{M}$, suivant toujours le ehemin de ronde depuis la tête du massif dont on a déjà parlé, insensiblement jusqu'à la eolomie $\mathbf{L}$. Cette colonne posée sur un rocher sert d'observatoire et de logement. Le dessous est un autre daus laquelle il y a des bancs en roches et garnis de mousse, et en partie de glaces qui répictent la prairie et la campagne. Des cliemins sont pratiqués dans les roches, et font monter sur une esplanade. En se pronenant antour de la colonne, on jouit de très-beaux points de vue. A quelques pas de la coloune, revenant au biatiment, on trouve deux chenins : l'un conduit au pont, et traverse la prairie; l'autre suit le bois, et revient au bâliment ou au potager.

\section{$\mathrm{N}^{\circ}$ 5o. JARDIN D'AGRÉMENT.}

Ce jardin contient cent trente-ciny arpens environ.

Le elıảleaı A est placé sur une élévation de six pieds au-dessus du niveau de la route.

Pour arriver au clïteau, on traverse une partic de forêt. On arrive à la grille d'entrée cqui est à eóté dı logenueıt du portier, et du lı-lıa désigné $\mathbf{B}$.

On traverse une prairie, plantée, sur les còtés, de maronniers. On passe devant une très-belle serre d'orangerie I, en avant de laruelle les orangers sont placés cn amplithéâtre; et sur le bord du chemin des masses de fleurs, qui se lient avec les orangers; ee qui fait un très-bel effet. De là on traverse la forèt, et on arrive au chấteau. Les roitures continuent leur route, passent devant le log̣ement du eoncierge, et vont aux ćcuries D, et de lá à la basse-cour E. Sur le devant du elıateau, du côté de la demi-luue G, on jouit du plus beau point de vue possible. Sur la droite du batiment, on a ponr point de vue une prairie plantée cle différens arbres.

Le ehemin de droile fait traverser la forèt, le vignoble planté d'arbres fruitiers, et on arrive au bátinıent du vigneron $\mathbf{K}$, qui fait point de vue au château. Le ha-ha, qui est sur le côté, laisse roir la plus belle forêt possible. A côté de la clıaumière est une porte de sortie. Différens chemins se présentent : les uns traversent le chemin qui va à la salle désignée $\mathrm{P}$, d’autres vont à la salle de repos $\mathrm{AI}$; d’autres rejoignent le chemin de ceinture.

Du bâtiment on a pour point de vue, du còté de l'entrée du jardin, un vallon assez profond, planté sur le devanl d'arbres verts, avec des unasses de fleurs en avant. Le temple do Diane V est élevé de douze pieds au-dessus du niveau de la prairie. Pour y arriver on traverse une forêt, une prairie plantée de groupes d'arbres, et entre ces groupes on apercoit la figure d'Apolloa Q, fils de Jupiter et de Latone, et frère de Diane, posée sur une éminence. L’autre chemin fait passer derrière la figurc dèsignée $Q$. 


\section{(53)}

Arrivé à une tèce de massif, le chomin de droitc conduit à une trés grande salle $\mathbb{I}$, entourée darbres

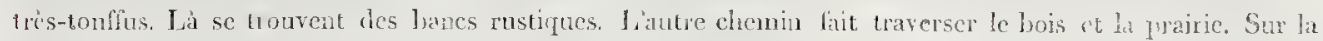
framie de droite est une fontaine antigne désignéc $O$, entourée de saules pleurcurs ct autres. Fn stritant le chenin on arrive à la salle désignée P, cutource de ljanes de gazon, deux ternes sout placés anx deus angles des massifs: ce licu est trés-solitairc. Le clemin de gauclie conduit au temple de Dianc $V$, et celui de droite fait traverser une parlie de foret, et dans me prairie se trouve places, sur un tertre, la figure. de Démophon Q, entourćc de massifs d'arbes. En surant la route, on arrive an lemple de Mars $R$, dien le la guerre, entouré da trés-grants arbres, faisant point de vue au temple de Diane $\mathrm{V}$. Un chemin y condait, et l'autre traverse le bois, passe derant un latha; de lí ou apercoit la forct. Ce mème chemin conduit at $11 u$ embranchument de chenn. Celui de gatuche conluit a une figure représentant Jupiter désigné $Y$, et l'antre va ḋue porte de sortic qui mène à la forèt. Un antre chemin conduit àl’obélisque X, qui est vu de temple $V$. Le rhemin de gauche traverse la prainic, et va au temple de Diane, lautre chemin lait traverser une forêt de luêtres. Arrivé à me tète de massif, le clsemin de ganehe fait descemtre dans une gorge plantée de pias ch sapins, et de là au bâtiment.

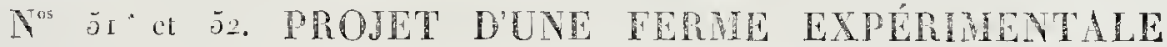

\section{DE JA ZOVE TORIJDE.}

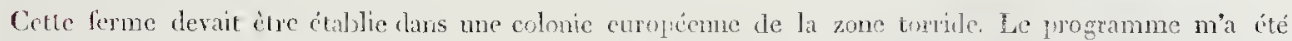
done par mon frère NI. André Thonin, memlere de l'Acadénic royale des Sciences, professenr d'agriculture an Muséun d'histoire naturelle, etc. (Jardin du Resi.)

Cette ferme serait ctablie dans un vallon entouse de montagnes du troisioue, deuxiène premier ordre.

Ce vallon, de forme oblongue, antait environ quatre nuilt: apens et serait traveise dans sa longueur par

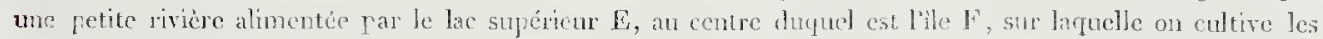
arbres [rmiers et antres; ]es caux du lac, ainsi que celles des sontecs lésignées $I$, toniberaient en cascades et feraicnt mouvoir des moulius à farine, a suere, à scies, à forges, à huile, ete. Aprés avoir quitué co bac ou ret étang qui servirait de vivier, de bain et de licu de natation, lu riviere formerait une ìle rers ie cerlre de la vallée doù chle s'échapprait ensuite. Des fontanes, des ruisseaux et des torrens réuniraicut cá ch lá leurs caux à celle de la riviere.

A. Ile $\left(n^{\circ} .52\right)$ contenaut cnyiron soixante-sis arpens.

(a) Eglise snscepilule de receroir douze d̀ quime cents persomes.

(b) Maison du direeteur de la ferme, du vice-directeur et ses bureanx; les corps de bátimont qui cntourent l'eglise sont destinés pour presloytere, logement de l'arditecte, l'inspectenr, mélecin, clirurgien, phonmaeicn, l'artiste vétérnaire, des easernes pon la tronpe, logement des domestiqnes et les jardiniers. Ises bâtimens qui borlent la rivière qui entoure lỉle sont destinés aux granges pour serrer les grains, les pailles et les fourages; des langars, des écuries, des toits à pores, des poulaillers, des colombicrs, ete,

(6) Jardins destinés pour la culture des légumes et fruits nécessaires aux personues logées dans charue maison.

(c) IIosjice pour les malades des deux sexes ponvant contenir cent cinquante lits environ.

(e) Vergers rl'arlores fruiticrs.

(i) Ponts agrestes pour ćtaljlir la communication de l'íle avce le vallon.

(g) Port couvert susceptible de recevoir une douzaine de bateaux pour les transports, dans les magasins, des récoltes cin tous genires.

Le terrain autour de l'église servirait pour lesmanouves destroupes et pionriers et à les exercer au maniement des armes.

B. (5i) Toutes les prices de bire antour de J'ile scrviraicnt, Io pour les haras de cheranx, de bouls, dadnes, de chameaux et mulets; $2^{\circ}$ de prairies pour les pâturages des troupeaur, des bétes à laines et à cornes; clies offriraicht des divisions et sulj divisions pour les sexes, les races, les àges ct pour les rúscrves des pàturages; 


\section{$\left.(5+)^{\prime}\right)$}

$3^{\circ}$ des prés pour foumili des fourrages secs propres a la nourriture des animanx pendaut la stison des pluies et celle de la sédieresse.

C. Pieces de terres destinces $I^{\circ}$ a une grande culture de liz, dans le voisinage le la riviere, afin cruon flisse arroser it volonte la rizere an moyen de petites deluses; si les arrosemens étaient ctablis par nappes,

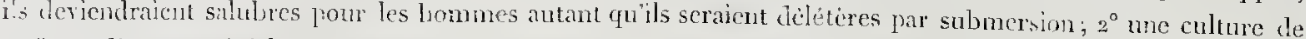

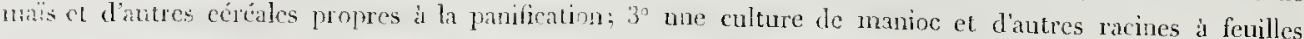

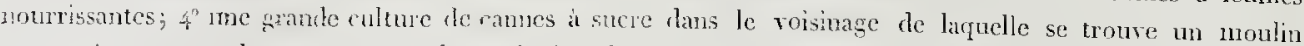
propre à pressure les cames pour la confection des sirops; 5 'une culture d’udigo herbacé diviscic en grands carrés de terres pourant che arrosés par irrigation loregu'il sera ncicessaire.

Les coteaux bordant le vallon seront diviés en stize partics dont chacune, afiectèt it une culture particulière,

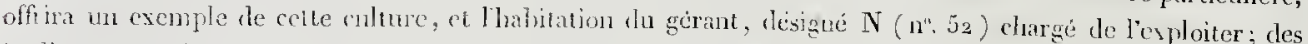
jardins susceptibles de produre le's legumes et les fruits necessaires à la nourriture de sa fanille; des bàtimens destincs i serrer les produits de culture, el des langars pour les outils aratoires.

Ces fermes particulieres, plus ou moins itentues, sclon l'espece et la nature de leur culture, seront:

r. Cotonneric onntenant environ quarante-rinq arpens.

s. Ene caferiere contenaut emiron cent cinquante.

3. Tuligotiere de lauriers roses des leinturiers, (ou nerium tiuctonitin, espéce nourclle) cent donze.

4. Lne sucrerie enterain hunile, envin tris cents.

5. Cacaotere, quatre cent cinquante:

6. Poivrerie, deus cent ingt-cine

5. Tailleric, cont cinquante.

8. Camclente, trois cents.

9. Girofferie, quatre cent cinquante.

1c. Huscaderie, ncul cents.

IT. Qunquinateric, quinze cents.

I. Conmeric (culture d'arbres fournissant des gommes), douze cents.

13. Résiueric (culture d'arbres fournissunt des resines), dis-huit eents.

1. Topalcrie culturc de nopalicrs, sur lesqucls sc nourrissent les cocheniles; clle doit être sur un coteau

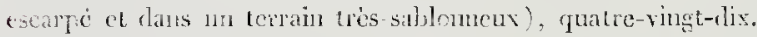

15. Pabuetrie (cilure des palniers dont on tire une seve propre à fournir des boissons fermentées), quinze cents.

16. Pejunicues propres à élever tous les jennes arbres destinés aux plantations de toutes les parties de la ferme, cmiron neuf conts.

A chaque culture il $y$ aurait des clemins concluisant à l'ile $A$.

D. (No 5r.) Montagies the second ortre, sur lesquelles sont etablies de grantes cultures d'arbres forestiers dé la zone torride, qui fournissent du bois à la clarpente rusale, aux Latimens cirils, it la marine, à la hate mature, au bardeau ou aux courcrtures des maisons agrestes; ì la menuiserie, ḋ la tomellerie, à la hoissellerie, au charromnge, à liubénisterie, a la marqueterie, au tour, etc. Autour du lac seroat de grandes cultures de mitriers cl d'autres arbres dont les fouilles sont propres ì la nourriture des vers á soie en plein air, sur les arbres ou dins des bîtimens G. Montagnes sur lesquelles sont quatre grandes cultures de naturalisalion des vegélaix des ropiques, qui, à ce degré d'èlevation, dojent pouroir s'acclimater sous la zone tonikle; ces cliverses cultures partageront toule l'étendue iles montagnes désignées $\mathrm{G}$; cluacune d'elle sera accompance de l'labitation du gérant désinne $\mathrm{N}\left(\mathrm{n}^{n}, 52\right)$, de sa famille, de ses ouvriers et de ses bestiaux.

1i. Les pertes des montagnes les plus hautes, jusqu'aux culloits courerts de neige et de glace permanentes seront occujeces par quatre grandes culures des végètanx des climats tempérés qui croissent sons les régions froides ct wione glaciales: le reste de la pente de ces montagnes sera divisée en pares destines à la multiplication libre des rigopnes, des linas, des chérres de eachenire, des condas et autres animaux de la chaîne des Corbillicres et de tous les climais froirts de la terre.

I. (N. 52.) Llération des moulins ilestimés à divers usages.

K. Sommet des montagues du premicr ordre; clles sont toujours couvertes de neige. 
1. ( $\mathrm{N}^{\circ}$. 52.) Seize bàtimens destinés au logement des gérans des cultures établies en $\mathrm{C}$.

M. Seize bâtimens destinés au logement des gérans des cultures établies sur les montagnes D.

N. Elévation en perspective des logemens et dépendances des gérans.

0. Cases pour les nègres ou autres ouvriers travaillant aux cultures.

\section{N.: 53. FABRIQUES POUR L'ORNEMENT DES JARDINS.}

1. Monument romain antiqne, en ruine.

2. Vase et ligure antiques.

3. Colonnade antique, en ruine.

\{. Temple romain en ruine.

5. Tombean antique, avec des trophées d'armes.

6. Fontaine antique.

7. Balancoire.

8. Rocher avec divers antres, sous lequel est une glacière, et surmonté d'un kiosque chinois.

9. Fontaine.

10. Fontaine.

11. Fontaine.

12. Tombean antique

13. Bâtiment hollandais.

If. Fontaine.

r5. Porte de jardin chinois.

16. Tombeau.

17. Porte gothique.

18. Tombeau.

19. Ermitage au milieu d'un vignoble.

20. Figure antique.

2. Cabane pour les cygnes.

22. Hangar pour abriter les bateanx et gondoles.

23. Volière

24. Balaucoire chinoise.

5. Figure antique.

26. Maison de pêcheur.

27. Volière chinoise.

28. Phare posé sur un rocher.

29. Bateau de pêcheur.

3o. Barque à voiles.

3 ז. Barque chinoise, au centre un pavillon.

33. Gondole.

\section{N. 54. FABRIQUES POUR L'ORNEMENT DES JARDINS.}

33. Pagode chinoise.

34. Figure antique.

35. Pont rustique fait avec des branches d'arbres.

36. Temple triomphal.

37. Figure antique.

38. Pont triomphal.

39. Jeu de bague.

fo. Pont chinois.

41. Pagodes turques.

43. Pont sumonté d'un are de trionphe.

43. Tour gothique. 
44. Pont égyptien.

45. Temple romain

46. Fontaine antique

47. Pont triomphal.

亿8. Vase antique.

49. Banc.

5o. Terme, logement dn fermier, des domestiques, écuries pour ler bestianx, granges four les fourrages, etc.

51. Obélisque égyptien.

5\%. Bàtiment chinois.

53. Jeu de bague chinois.

54. Banc.

55. Jen de bague rustiçue.

56. Monument gothiqne.

57. Tour ehinoise.

58. Fauteuil de jardin.

59. Maison đle bûcherou

Go. Chaise.

61. Figure antique.

62. Chaise

63. Tente turque.

64. Maison de meuniers el moulin it au

65. Figure antirue.

66. Banc rustique.

67. Banc chinois.

68. Banc tarc.

69. Figure anticne posée sur un piédestal.

\section{$\mathrm{N}^{\circ}$. 55. PLANS DES FABRIQUES POUR LORNEMENT DES JARDINS.}

Celte plauche est composée des plans des fabriques des planches 53 et 54 .

\section{N. 56. FABRIQUES POUR LORNEMENT DES JARDJAS.}

1, 2, 3, 4, 8 et 9. Maisons rnstiques couvertes en paille.

I0. Salle de danse couverte en paille.

11. Haison mstique couverte en paile, servant de salle de billard.

15. Maison rustique hollandaise.

16 et 17. Salle de danse couverte en paille; les colonnes seraient de bois en grume.

$18,22,23,24,25,28,29,30,3$. Temples et bâtimens variés. (Ils peuvent être établis en plàtre, ct les décorations extérieures seraient peintes à fresque.) Its servitaient de lieu de repos et d'ornement des jurdins. Toutes ces fabriques ont leurs plans, une échelle pour l'élévation et une ponr te plan.

Les onze nodèles de treillage sont exécutés, au Jardin du Roi, d'après les plans de M. Molinos, architecte.

\section{$N^{\circ}$. 57. JARDIN CHAMPÊTRE,}

Contenant 200 arpens environ avec les parties extérieures.

Le bâtiment se trouve placé dans un fond, apercu de la grande roule, à la distance d'une lieue.

Du bàtiment, on a pour point de vae d'immenses prairies, divisécs par deux rivières, l'une supérieure, qui fait tourner deux moulins.

\section{PROMENADE}

Le chemin jurès du bàtiment, désigné $n^{\circ}$. 1 , fait traverser un pont; à droite de ce pont, est une ile plantée de toutes les espèces d'arbres et arbustes de terre de brayère; on a aussi pour point de vue un déversoir, 


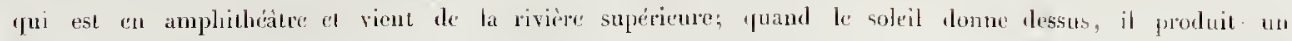
tffel admirable.

Plus loin est un second pont vis-ì-tis le chemin nº a, lequel chemin monte rapidement, traverse les pins, sapins, des roches, et, á mi-cốte, se trouve une tigure rẹrésentant. Héléagre, $n^{\circ}$. 3.

Le chennin de gauche, arrivé an $n^{\circ} .4$, conlnit à une salle de danse et de différens jén, comme halancoire, etc

$\mathrm{N}^{3}$. 5. Chemin trave:saut la forît el conduisaut au potager, tírigué M.

$N^{\circ}$. 6. Chemin conduisant par une pente donce à l'ermitage, désigné L.

Revenant an $n^{\circ} .3$, le cluenin de droite arrive à une grille, d'oú l'on va au village désigné I. 'T'rois chemins vont se joindre alı $\mathrm{n}^{\circ} .7$, où passe le chemin de ronde, ‘ui conduit à un pont inmense désigné $\mathbf{C}$, construit avec des troncs d'arbres, qui lient les deux montagnes ensemble; l'hiver, un torrent passe sous ce pont, oú l'on a creusé et recouver en maçonnerie une allée du potager pour l'éconlennent des eaux. Cús eaux ront se jeter dans liz rivière supérieure.

Passé ce pont, le cliemin de gauche traverse la forêt et vient au temple de Jupiter, désigné $\mathrm{N}$, léqriel est posé sur un rocher très-escarpé; des clemins traversent des roches qui ont été culbuties par le tenros. De cel endroit on découvre une prairie émaillée d'arbres à fleurs et à odeur, et des rivièrés qui serpentent dans ladite prairie.

Dudit templé, on a aussi à traverser de trés-Jbelles masses d'arbres et des prairies; nue maison gothique, clésignce O, sert de logement pour un garde et de point de rue au temple.

Le chemin, $n^{\circ} .8$, fait traverser les arbres fruitiers, les céréales, el va au moulin désigné $P$.

Revenant sur le cláteau, rons suivez une chamante riviére bordéc de différens arbies, el arrivé an nºg, vons montez par un cliemin doux à travers lus céréales, groseilliers et arbres fruitiers, et qui aboutit à la maison In garde, désignée 0 .

Le chemin qui suit le bord de la riviére arrive au pont désigné ro. Ce pont en grume, trés-élevé, fait traversol de l'une à l'autrc prairie.

De ce pont, on a pour point de vue un lac, avec deux iles plantées de roseanx, ou, l'hiver, on fait la chasse aux canards sanvages.

le chemin, $n^{\circ}$. 11, monts rapidement, fait traverser des pins, des sapins, des roches, et va rejoindre le temple de Jupiter, désigné $\mathbf{N}$

Le chenin, $\mathbf{n}^{\circ}$. 12 , suit le bas de la riviére, traverse d'inumenses peupliers d'Talie, de Suisse, de Mollande, etc. de toutes les variétés, et va rejoindre le pont, r". 2 ; eu suivant ce clemin, vous arrivez successivement à trois endroits, au village désigné $\mathbf{I}$, à la ferme désignée $\mathbf{K}$, et au château $\mathbf{C}$.

P'rès du chàteau, promenade; suivant le bord de la riviére vous arrivéz à un pont en roche, ñ. 13, d'où l'eau sort arec impétuosité de quatre pieds de hauteur, dans un bras de rivière, et vient regagner le tour de l'ile plantée d'arbres et arbustes de terre de bruyure.

Da pont, vous avez un chemin qui fait traverser lỉle de Flore, plantée de toutes espréces de rosiers et autres arbres à fleurs variées, et vous arrivez à un rocher désigné $\mathrm{Q}$, sous lequel on met les bakaux á l'abri.

Passé l'île, est un chemin suivant le lac planté de saules-pleureurs, peupliers, catalpas, etc., el qui va rejoindre le pont, 11 . 13 .

Le chemin, i3, suit l'autre bord du lae et va rejoindre un pont chinois, n. 14, posé sur des roches. Ce pont. vous fait passer daus l'ile d'Amour.

A la tète de l'ìle, est une figure représentant l'Amour, et à l'extrémité le temple de la dicinité de l'ìle, R.

Celte île est plantée des arbres les plus rares, comme halmia, rbododendrum, azalea, magnolia, etc.

Un autre pont fait sortir de l'ìle, suit le bord de la rivière basse, et rient regagner le grand pont, n'. 10.

Passé le pont, le chemin conduit à la salle des grands houmes, désiguée 'T, et de cette salle on décourre une charmante prairie et le temple de Neptune, S.

Le chemin en face du pont traverse nne immense forùt, des parties de prairies, et vient au temple de Diane, U, servant de point de vue à la salle des grands hommes.

Suivant ce mème chemin, traversant toujours la forêt, vous arrivez á la rencontre de deux chemins; l'un conduit à celıi désigné no $\mathbf{r} 4$, qui traverse un verger é ra rejoindre la salle des grands hommes, $\mathbf{T}$.

No. 15. Allée d'orangers.

$\mathbf{N}^{n}$. i6. Allée plantée à droite et á gauche de petits pommiers-praradis et de rosiers, pour ne pas priver les orangers du soleil et afinde masquer seulement la murailte qui sontient la terrasse sur laquelle sont placés ces orangers.

A l'cxtrémité du petit clremin, est l'orangerie, $G$, la serre chaude $H$, ensuite la glacière, $V$, et un endroit jou1 déposer les terres, $n^{n} .17$.

No. 18. Chemin conduisant á la maison dı garde, IV.

$\mathrm{N}^{\circ}$. I9. Clemin traversant la grande forèt et allant joindre le bàtiment.

$\mathrm{N}^{\circ}$. 20. Chomin supérieur, planté de très-grands arbrés.

$N^{\circ}$. 21. Chemin descendant asse\% rapidement, allant à la salle des grands hommes el rejoignant ensuitc le bàtiment. 
N". 22. Chemin traversant le viguoble, les arbres fruitiers, et allant au moulin désigné $P$.

Le chemin qui est en face du pont ou du moulin monte trés-rapidement et vient regagner le chemnin supérieu et anssi celui qui arrive à la salle des grands hommes, désignée $\mathbf{T}$.

1. Grande route.

B. Entrè du château.

C. Château.

[3. Cour, Remises, Ecuries.

E. Conr d'honneur.

F. Communs.

G. Serre dorangerie.

H. Serre cliande.

l. Village.

K. Ferme.

L. Ermitage

H. Potager.

V. Temple de Jujiter.

O. Maison d'un garde.

P. Maison thun meunicr.

Q. Roche pour mettre des bateaus a l'abri.

R. Temple de l'Amour.

S. "Temple d" Neptune.

T. Salle des grands hommes.

U. Temple de Diane.

$\checkmark$. Moulin extérieur faisant point de vne au chiteau.

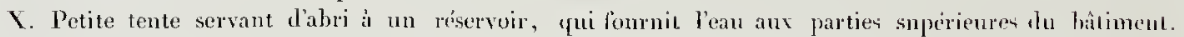

I. Grand lac.

A. Entrée par le village.

\section{No. 58. JARDIN PITTORESOUE.}

E. Etang.

C. l'ernw, remises el cicuries.

1). Vignoble.

E. Etang.

li. Tenple de Neptune.

G. Ile d'Mnour. Les jonts peuvent itre deux bateaus tenus par des crocbets; on se sert de ces bateaux pour se pronener sur l'étang; an centre de l'ile, me figure do l'Anour, 6.

11. Banc en roche avec un sanle-pleureur, qui met it labri du soleil.

L. Polager qui se lie avec le verger.

K. Verger.

L. Bâtiment ayant quatre points de vue :

Le premier sur la droite. On voit la campagne ayant en soin de n'y plauter que des arbres qui ne dépassent que trés-peu la muraille, pour deux raisons : l'abord pour la vue, ensuite pour ne pas donner d'ombre à l'espalier et au potager de gros légumes.

Le deuxième poiut de vue est sur un très-beau village, $n^{0} .2$.

Le troisieme point de vue, sur le temple d’Fole, $\mathrm{u}^{\circ} .3$, posé sur une éminence de 5 pieds; au-delá de ce temple, ou décourre la campagne.

Le quatrième point de vae est le còté du colombier $u^{\circ}$. 4, et du village.

On a eu soin d'éloigner les bàtinens des remises é écuries, pour éviter le mauvais air et afin que le bàtiment seit entouré de verdure.

M. Entrée de la grande ronte.

N. Jeu de bague.

O. Allée doó l'on jouit d'un trés-bean point de vne sur la campagne.

P. Balancoire.

Q. Salle de danse.

R. Tapecu.

S. Sortie sur la campagne.

T. Temple al'Eule. 


\section{है}

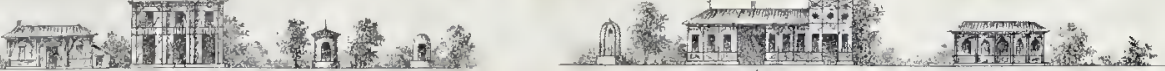

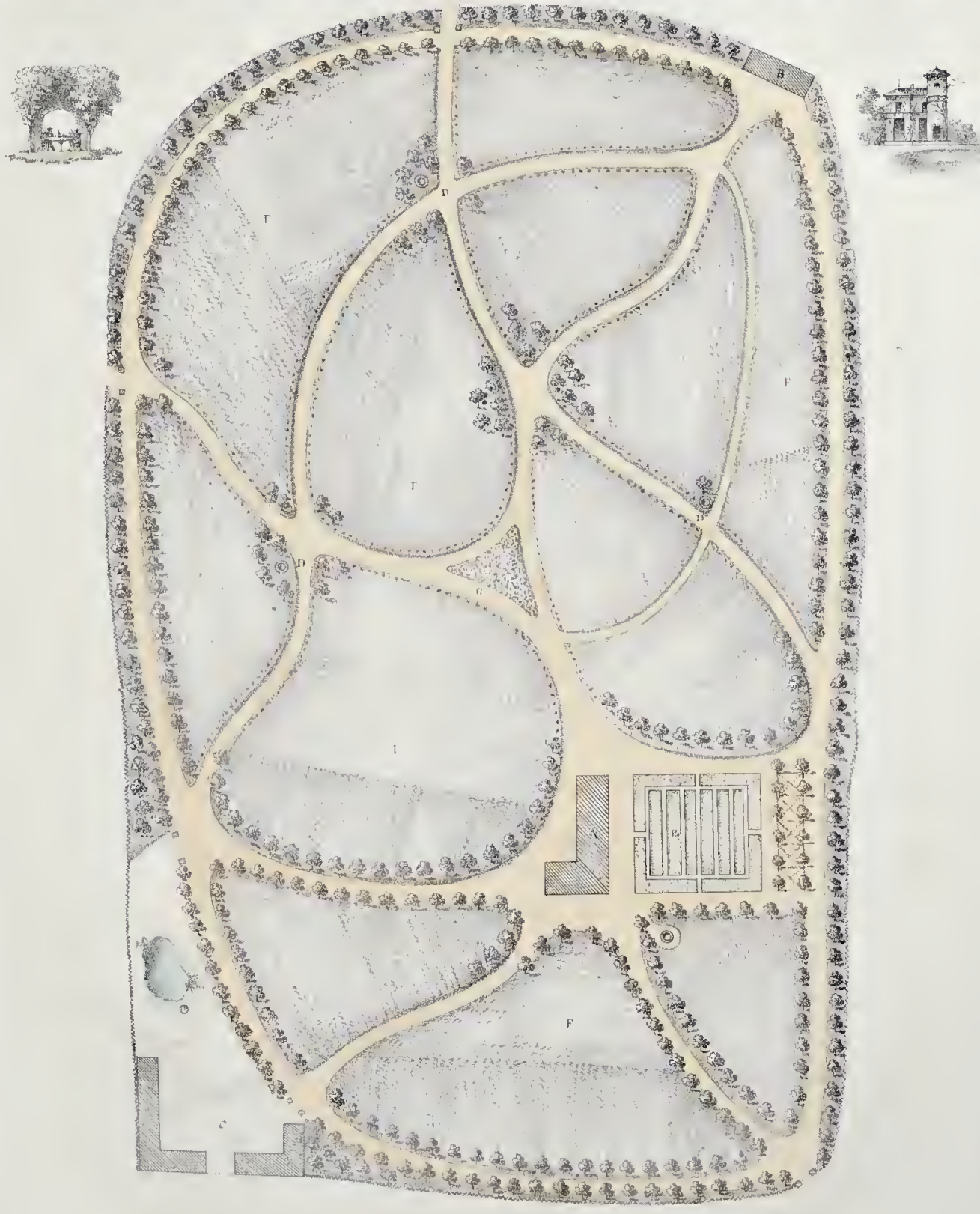

Srentire de gros légumes. 


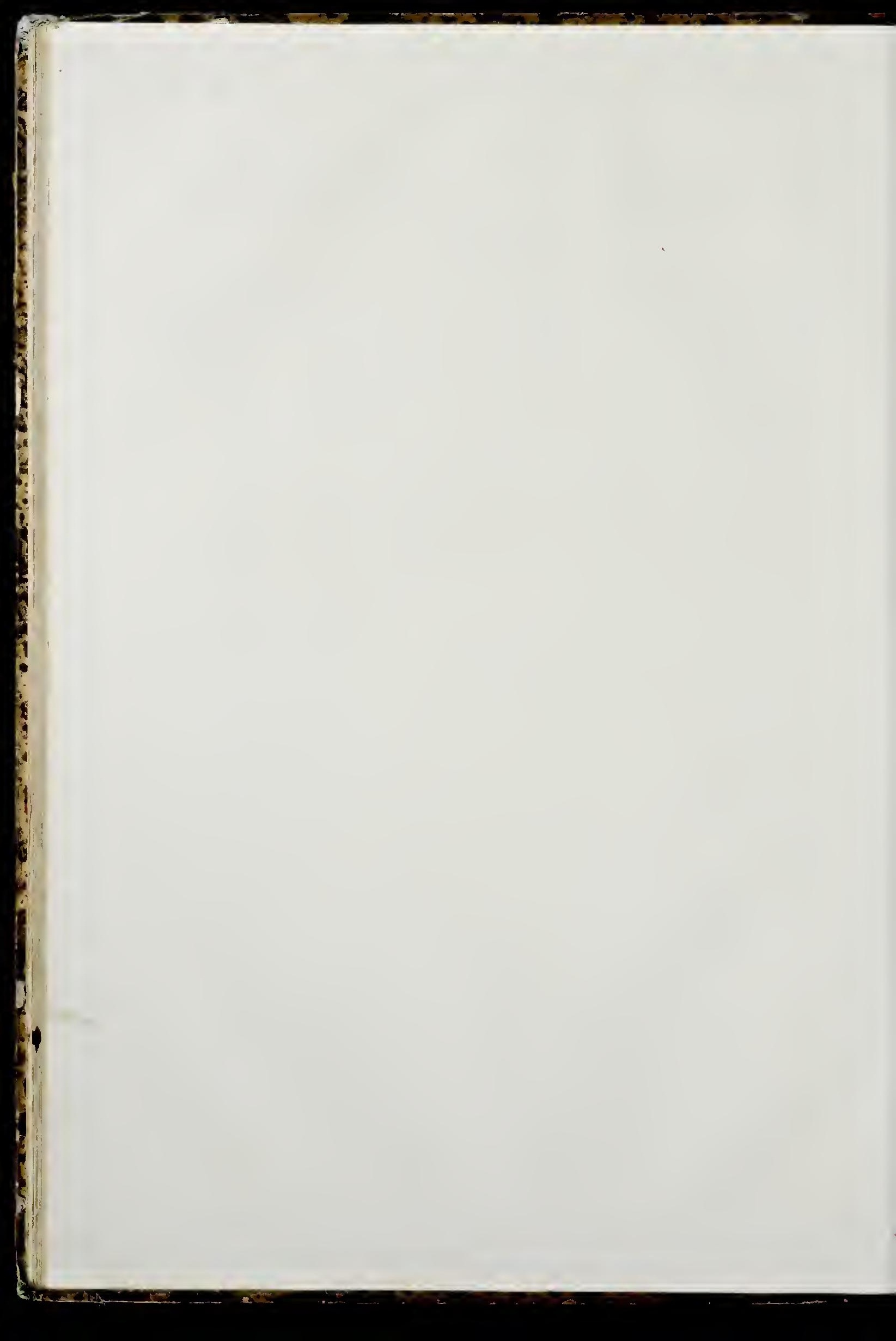




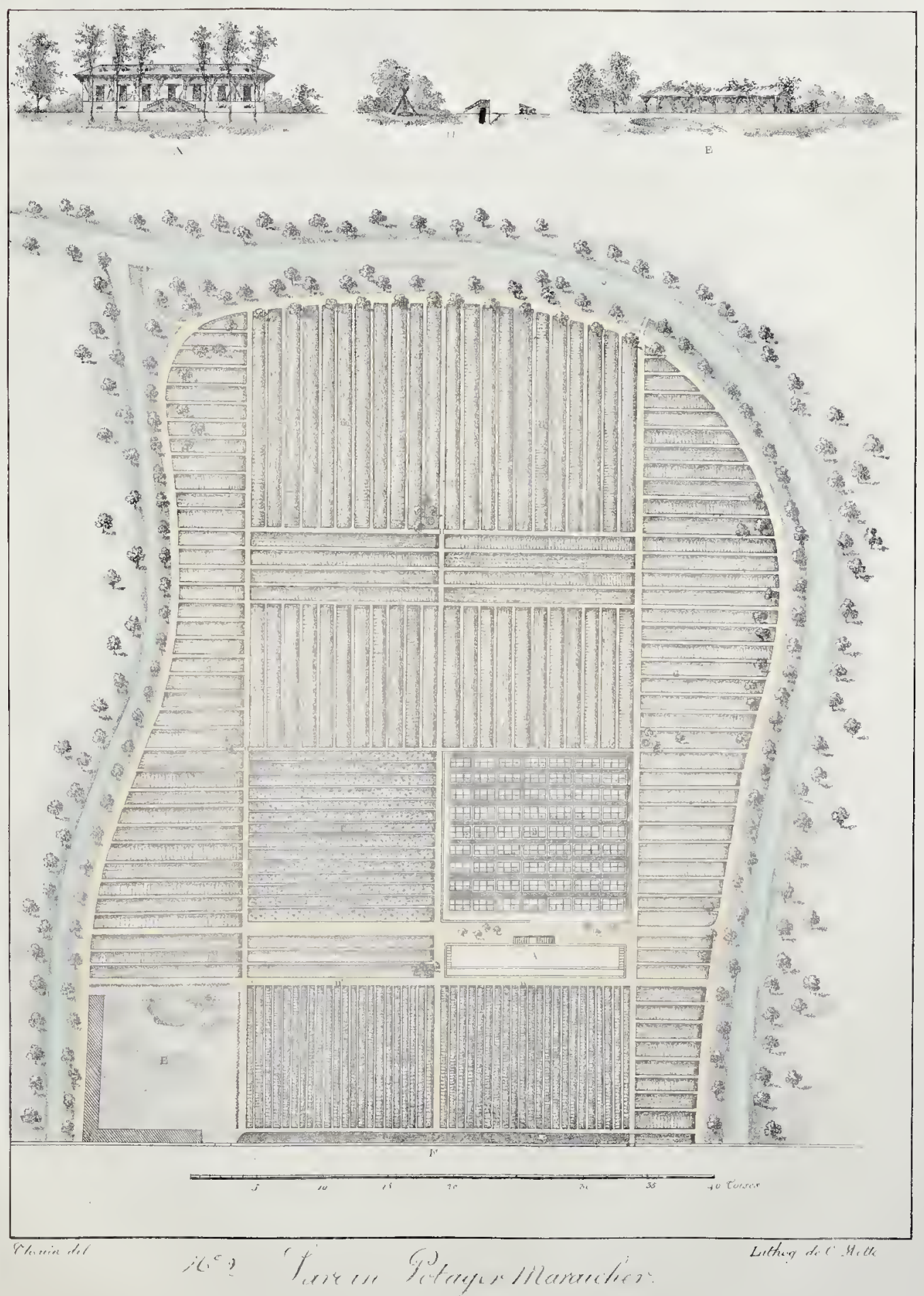




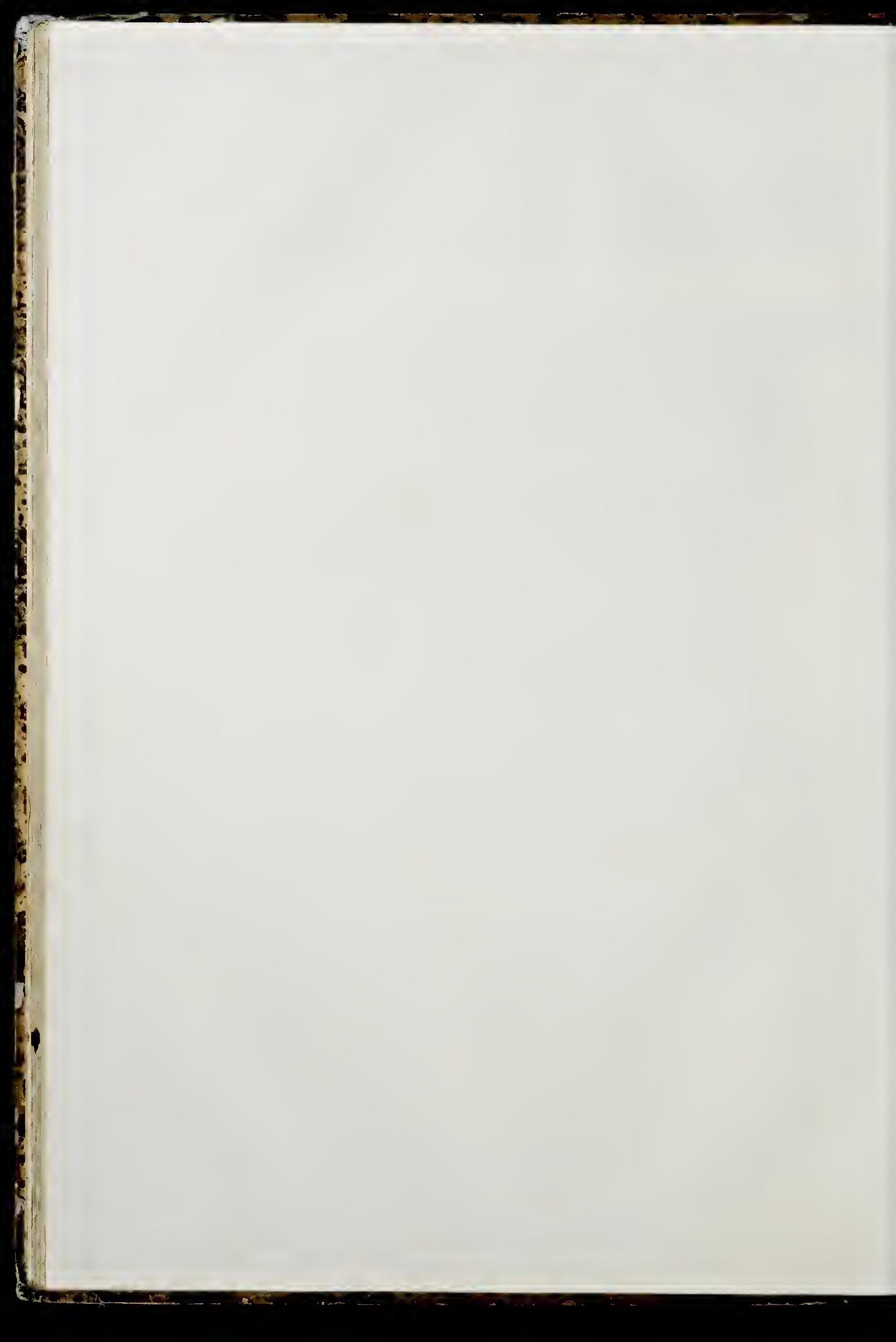




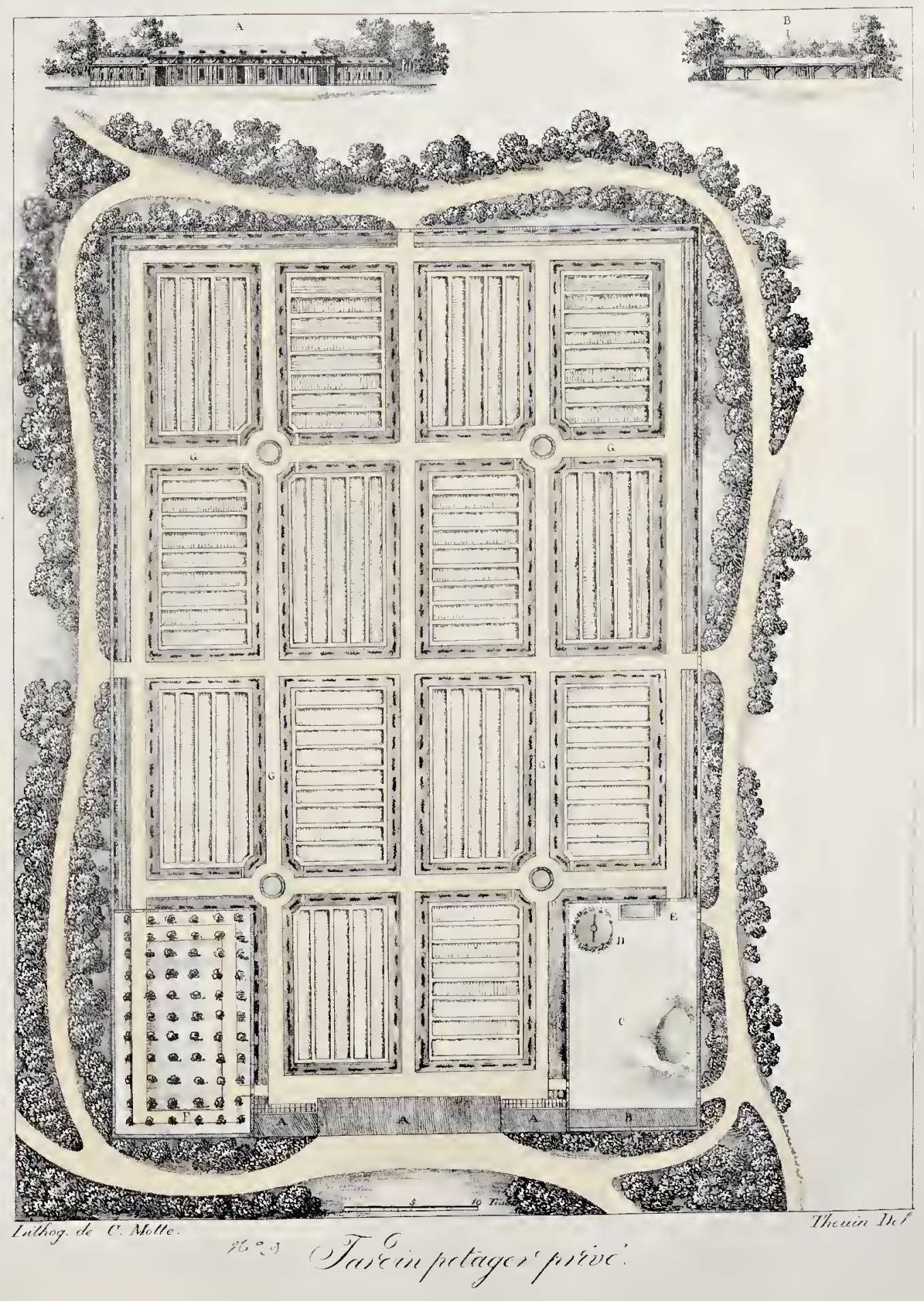




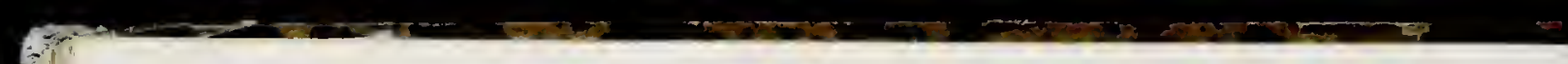




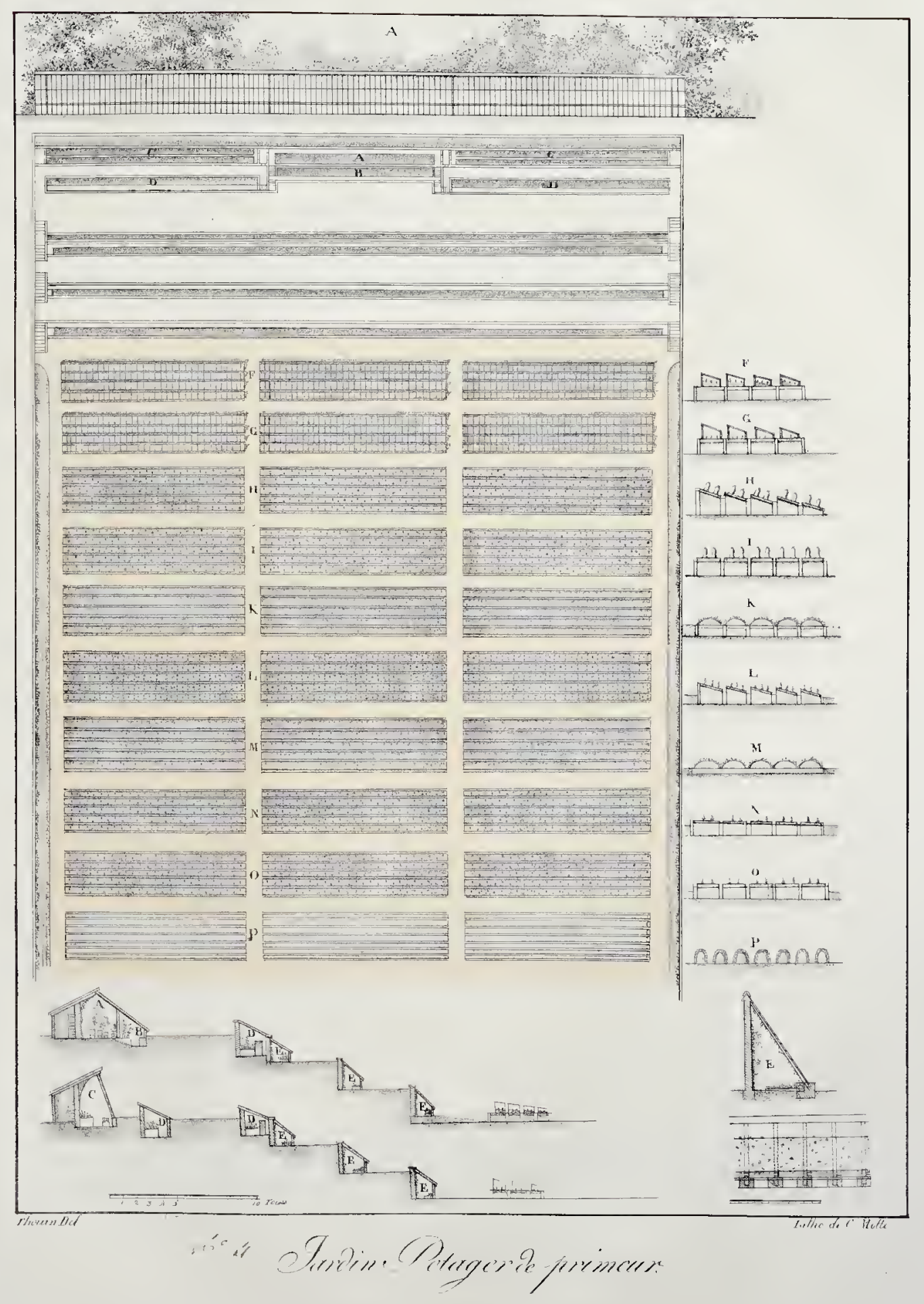




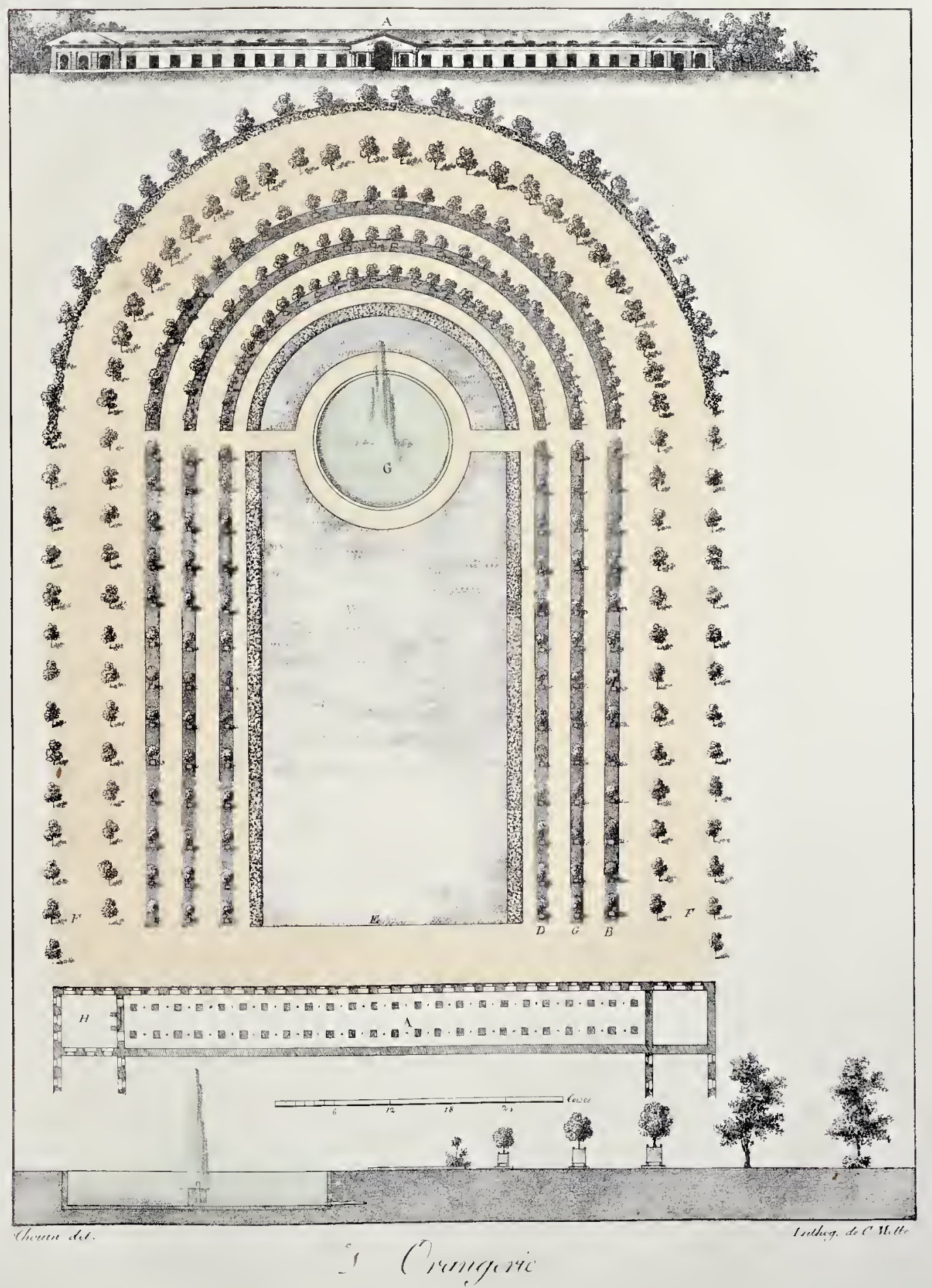




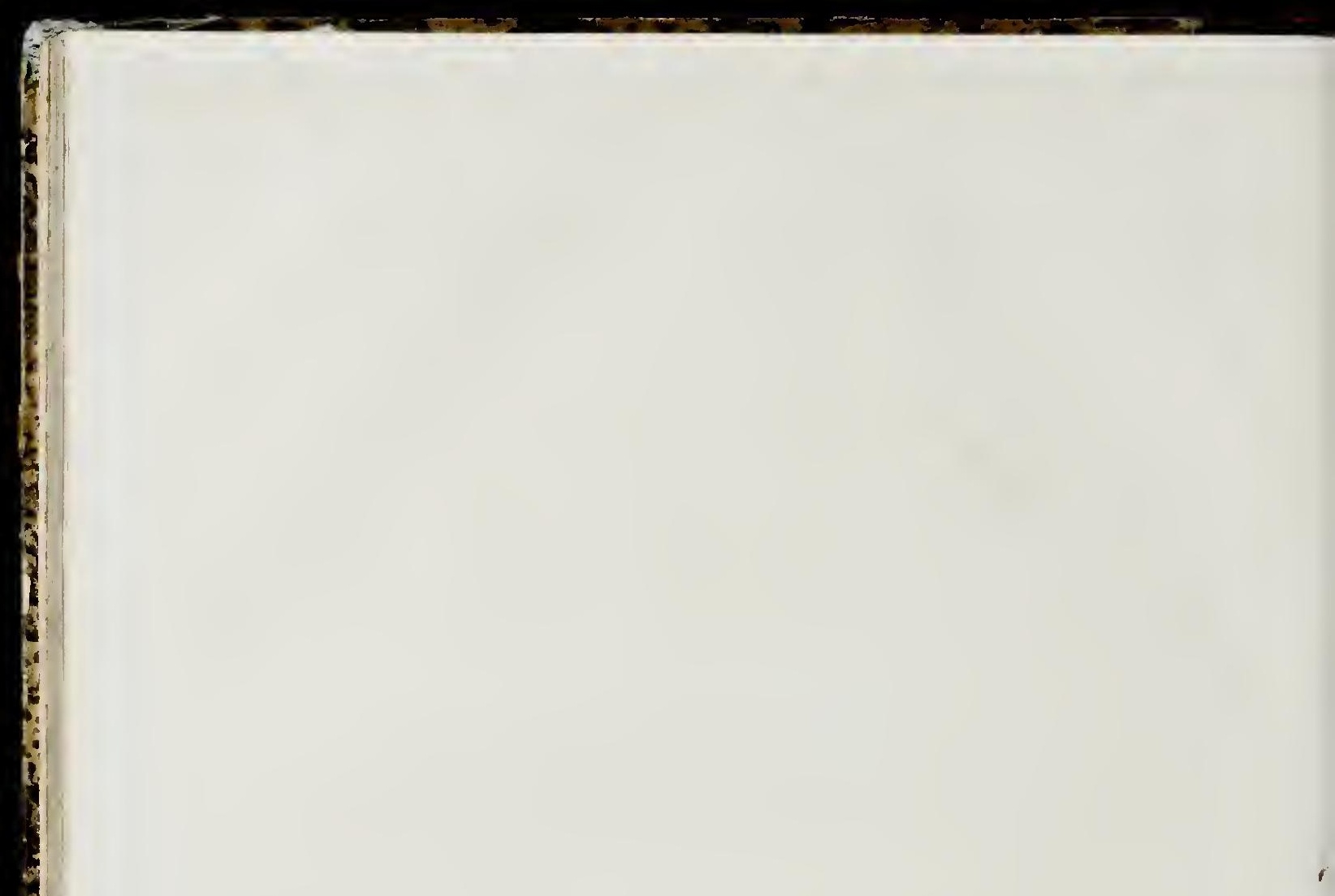




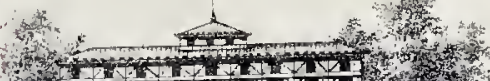
a

\section{7i}

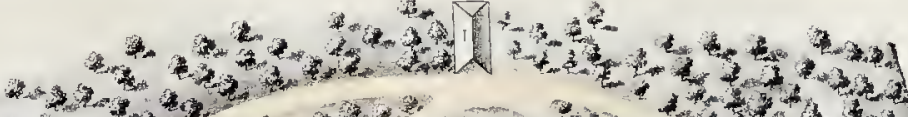

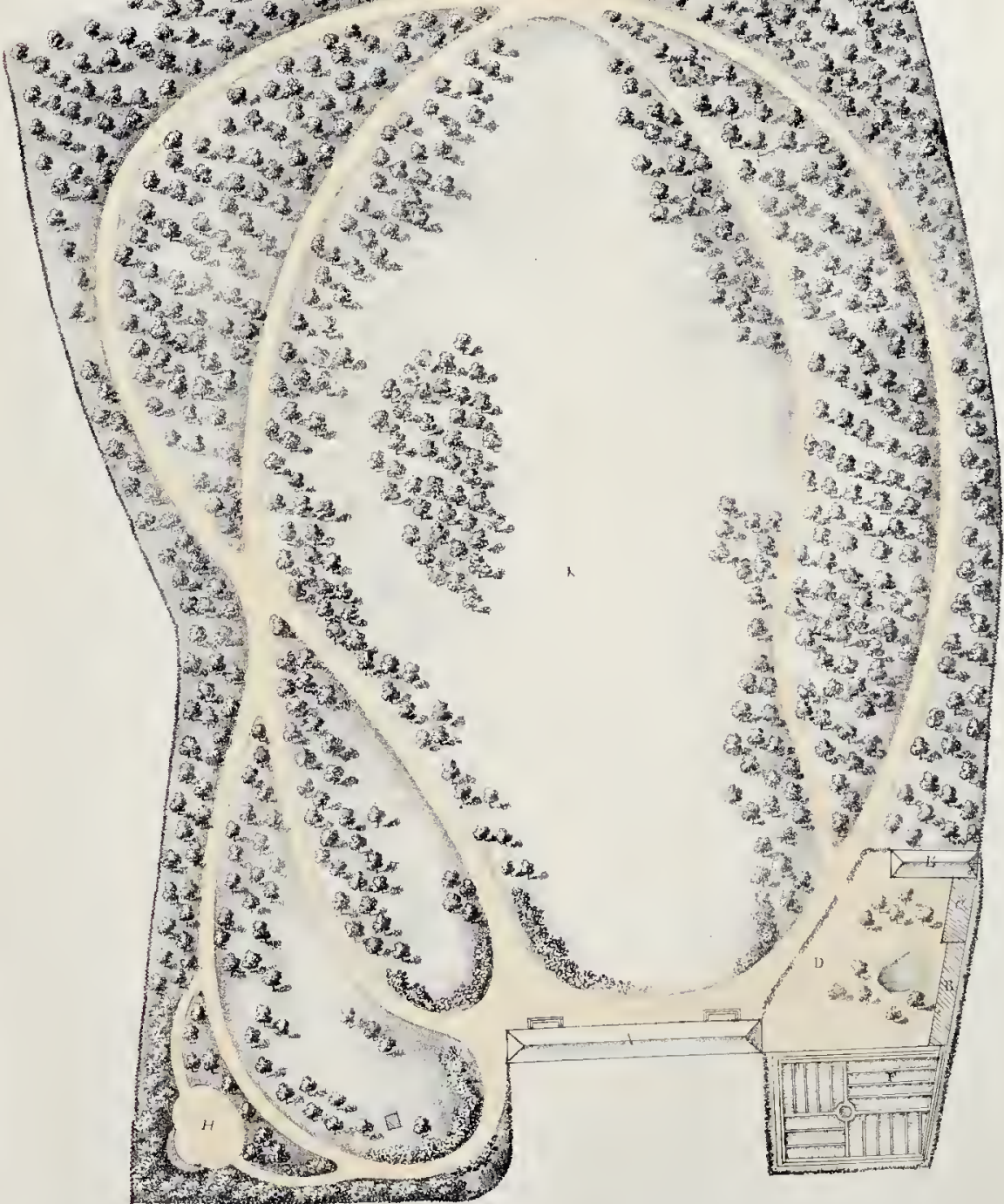

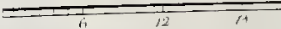




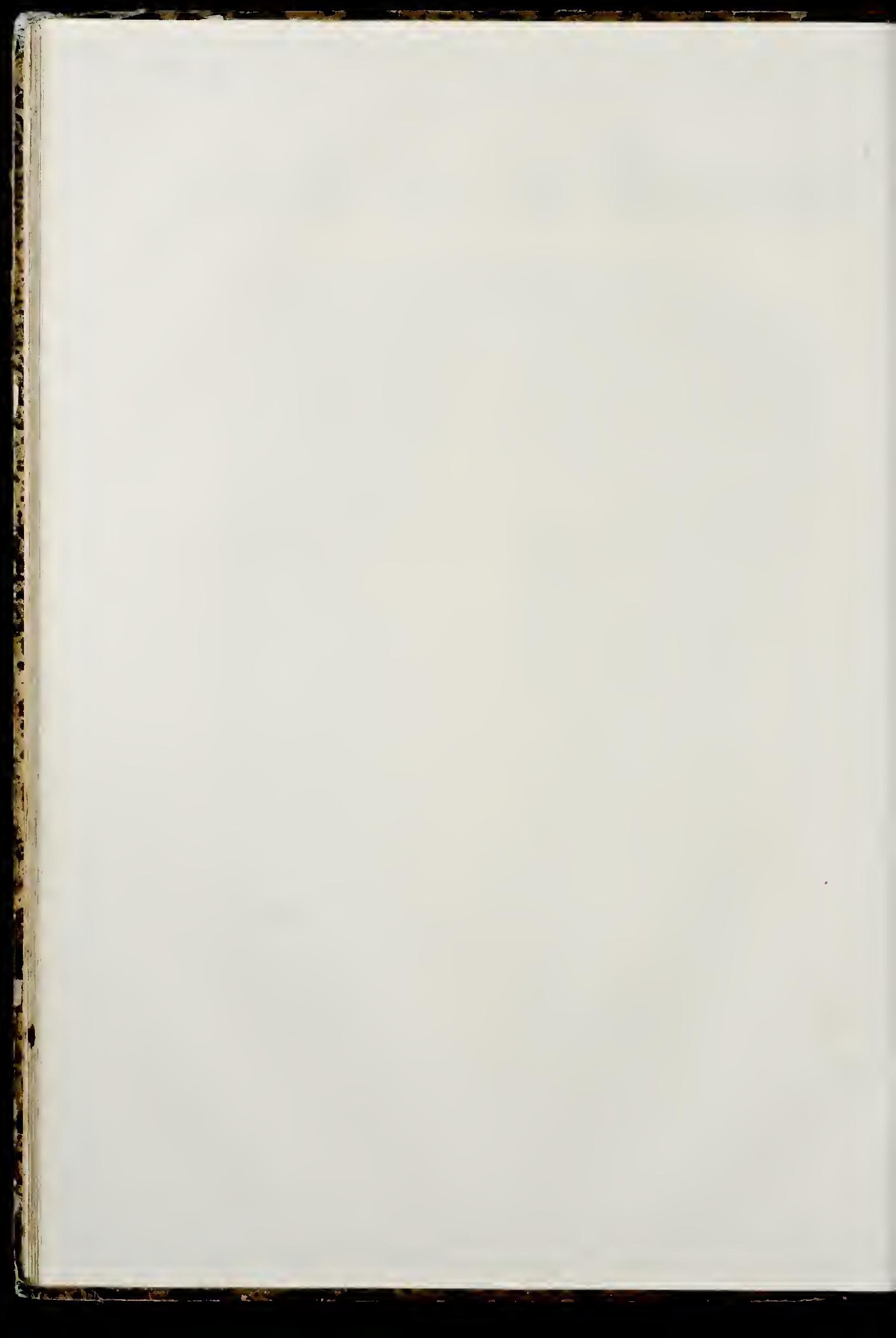




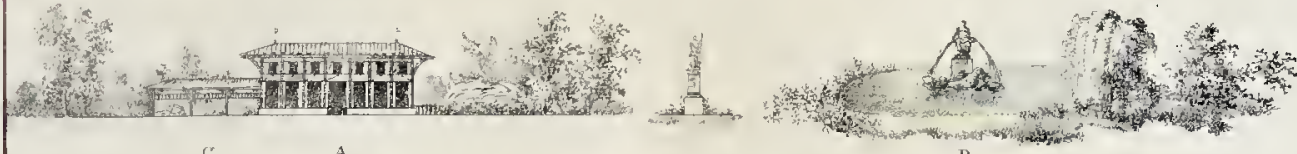

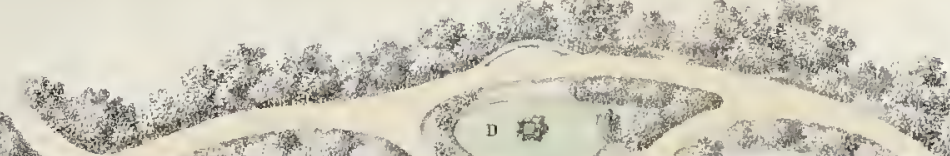

H.

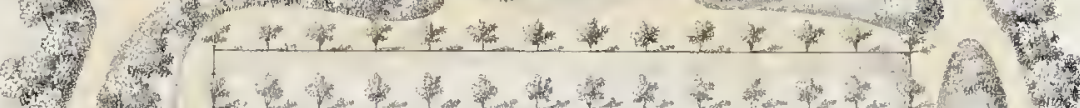

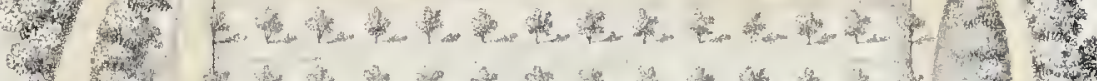

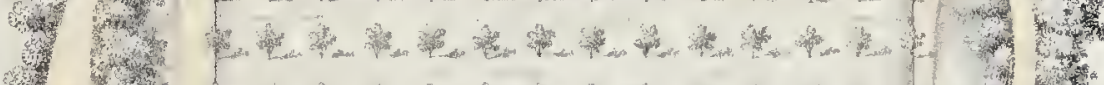

(3)

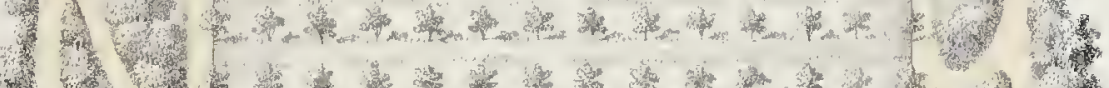

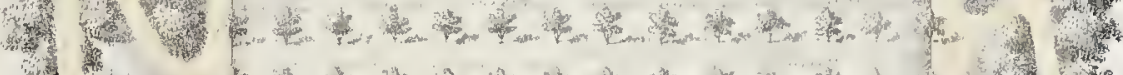

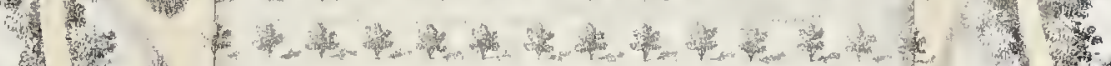

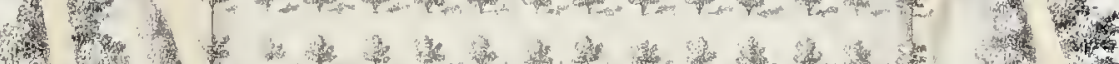

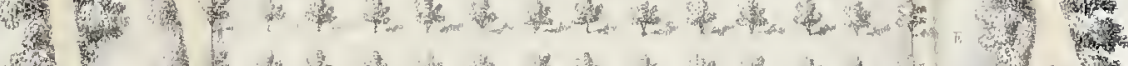

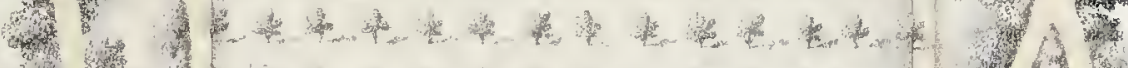

(5.

of

.

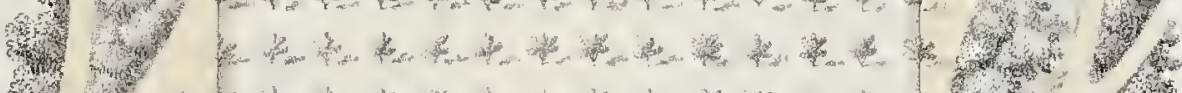

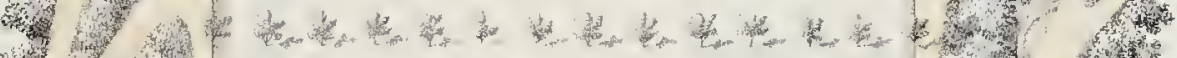

(4)

1)

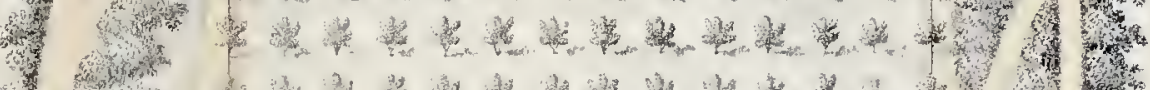

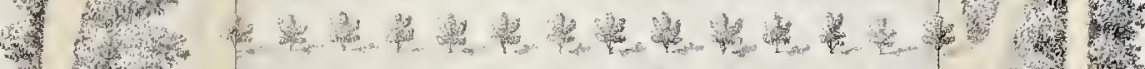

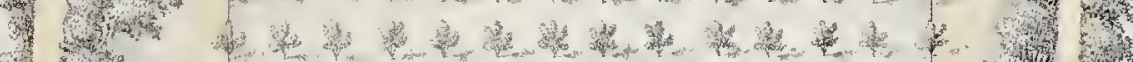

Mr

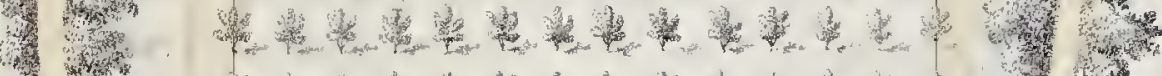

3.

4 .

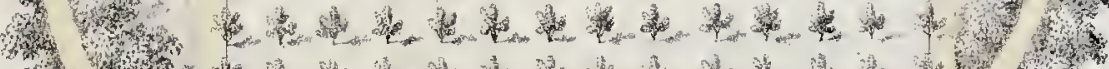

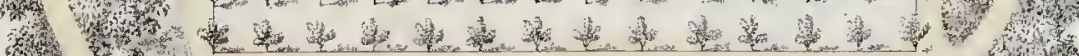

(t)

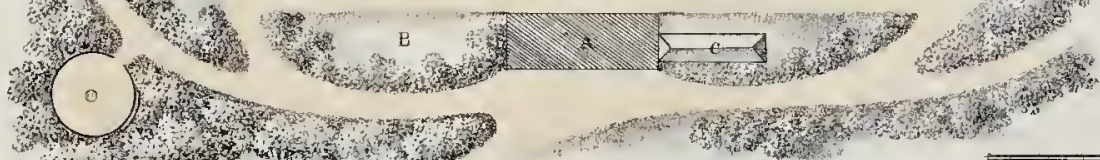
1.

Thowen Del.

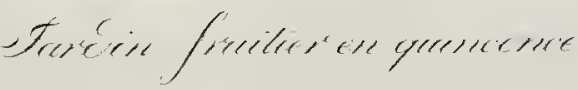




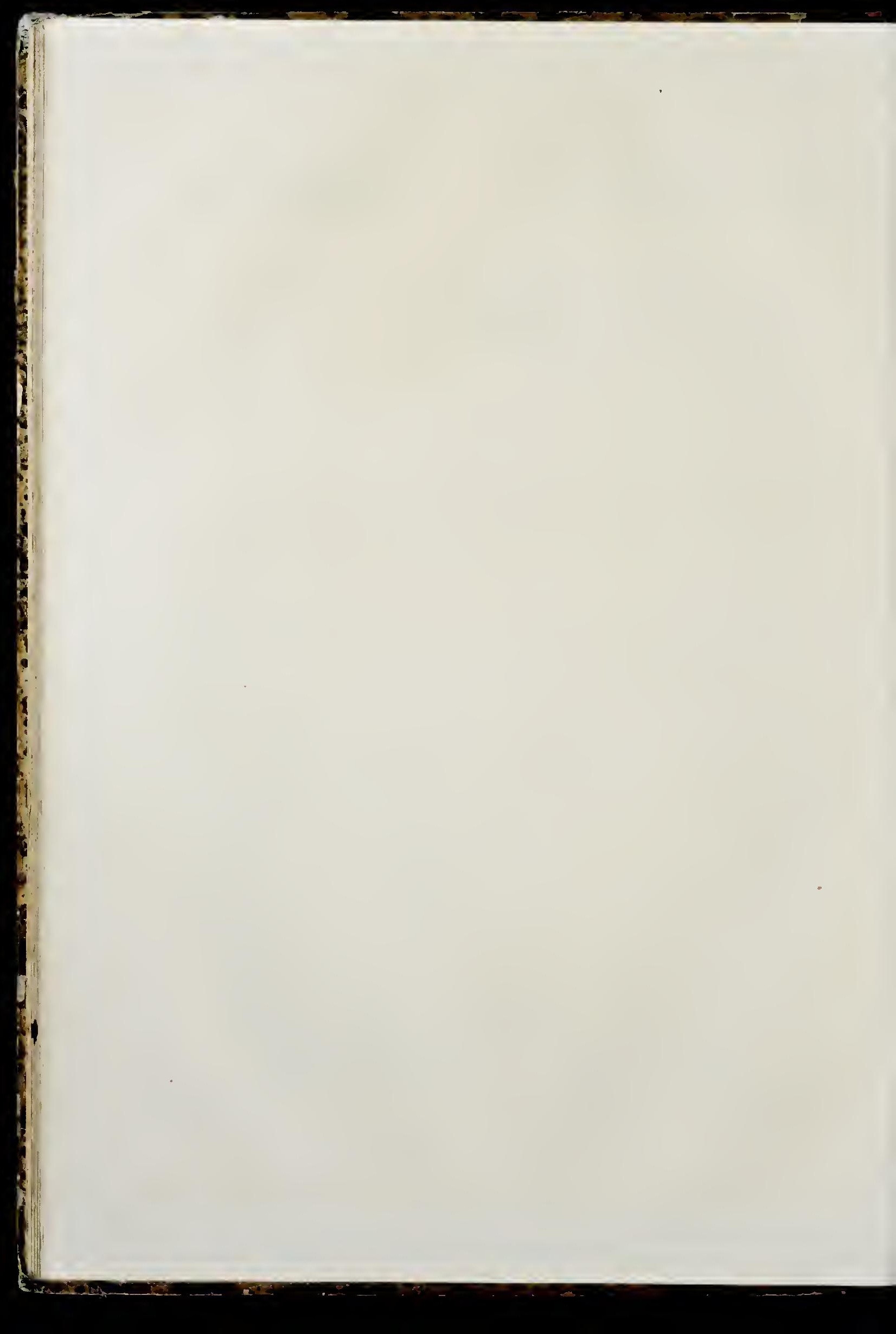




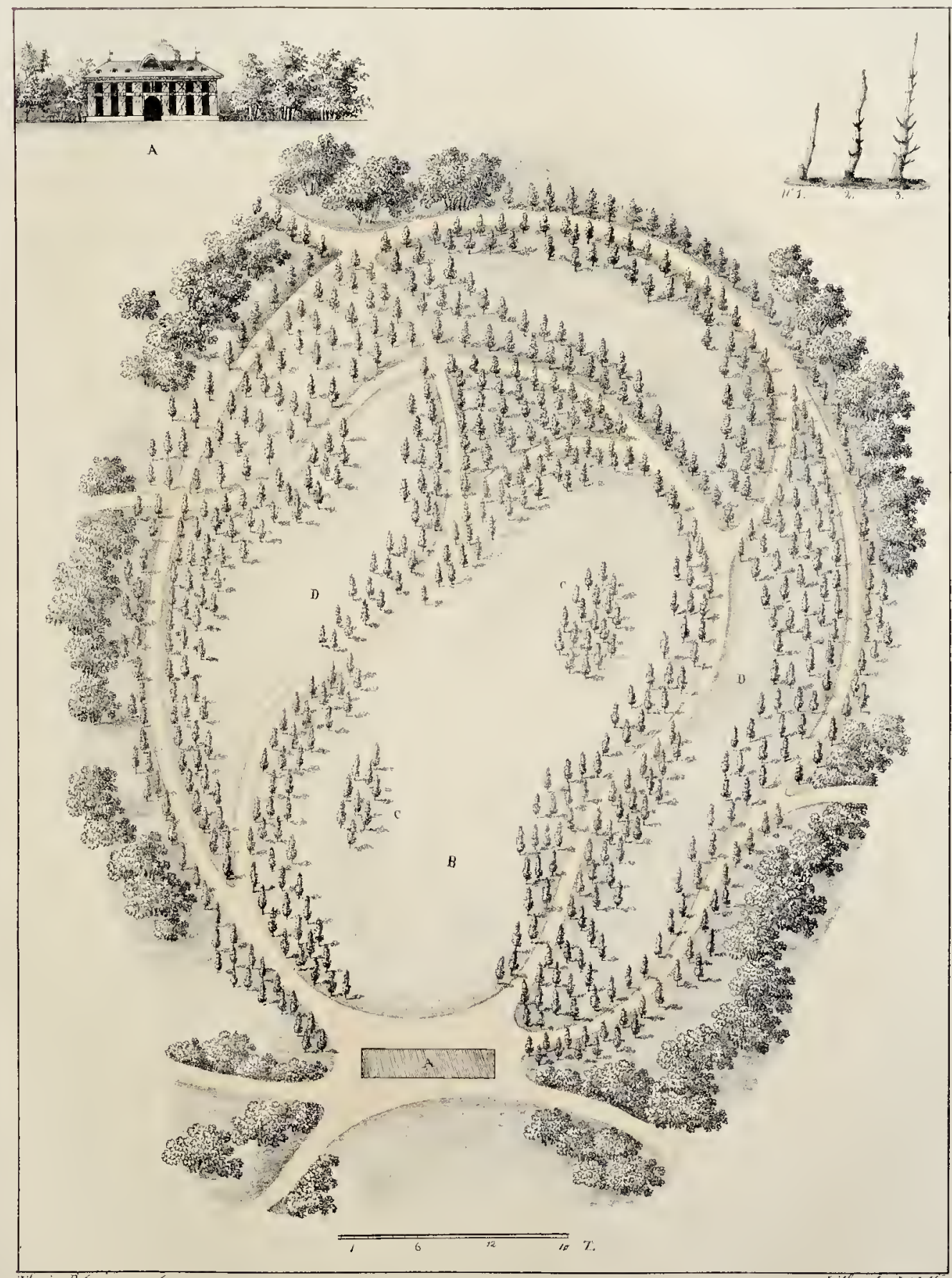

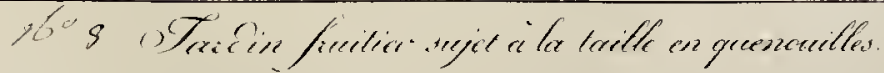




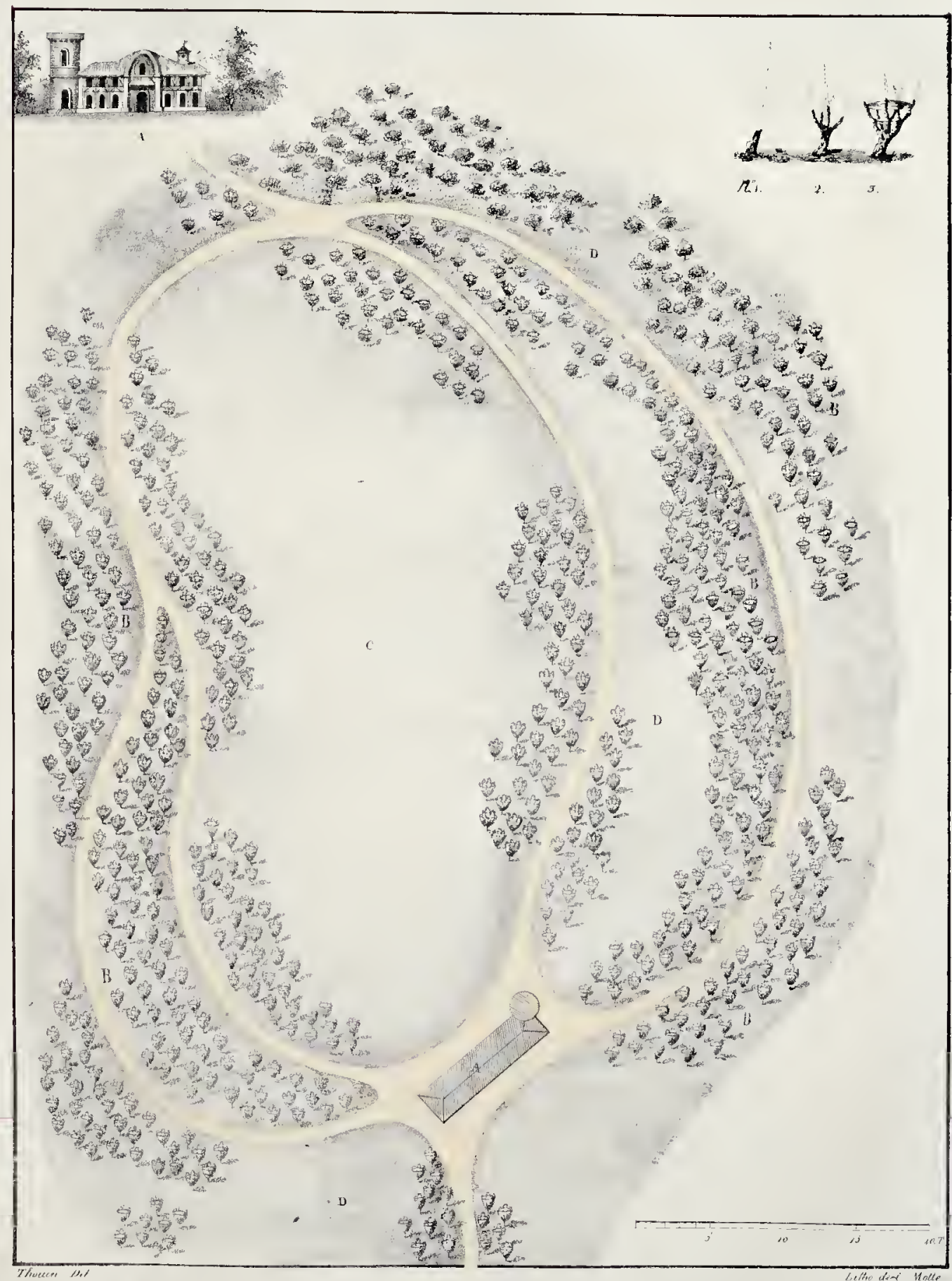

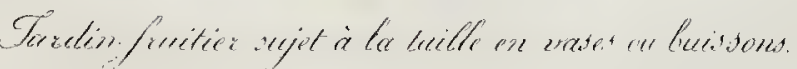




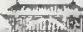

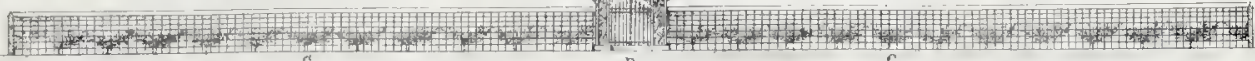
क्रxम
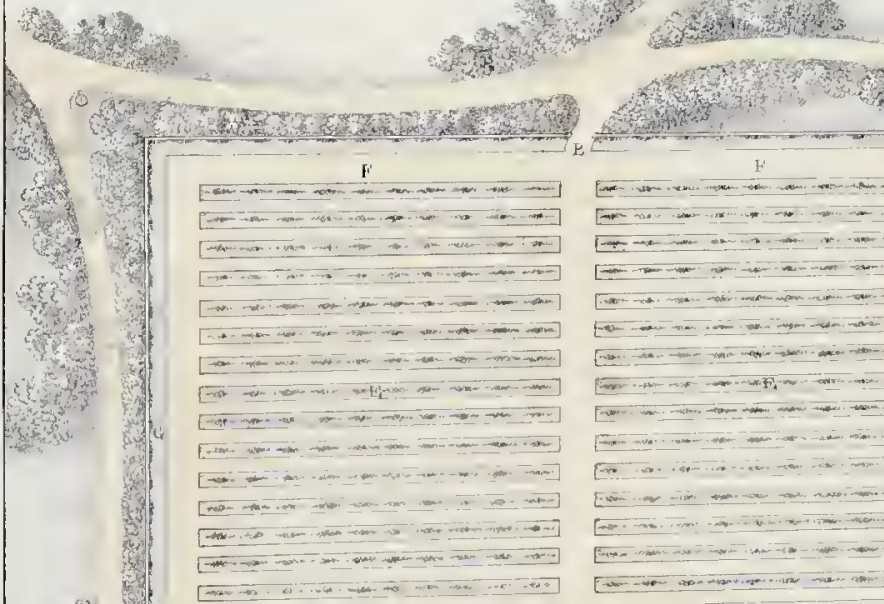

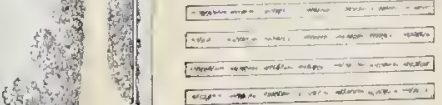

(1)

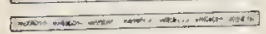

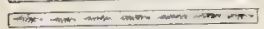
- $-m-\infty$

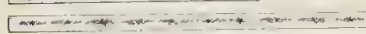

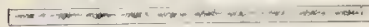

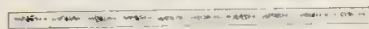

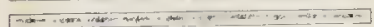
and Ex -

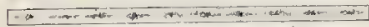

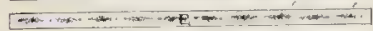

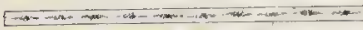

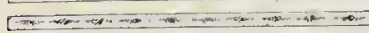

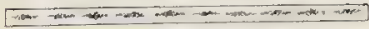

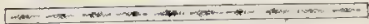

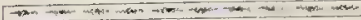

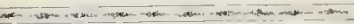
$-\infty-\ldots+\cdots$ $-\cdots+\infty-1+\infty$ [-x- -

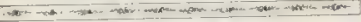

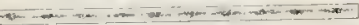
tor

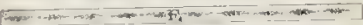

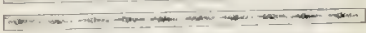

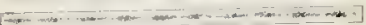

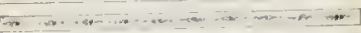

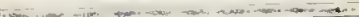
[O.

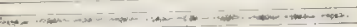

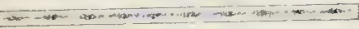
E....... $-\infty-1-1$ [an

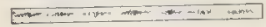
(3)

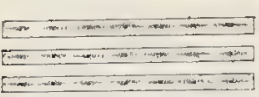

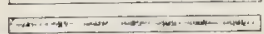

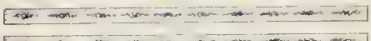

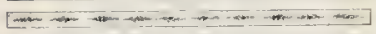

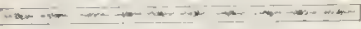

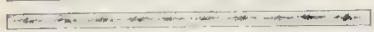

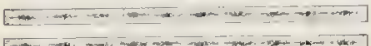

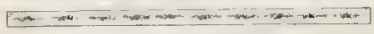

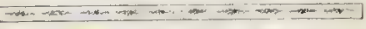

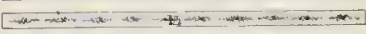

$+\rightarrow-\infty+\cdots$

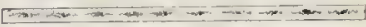

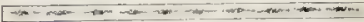

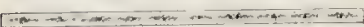

-

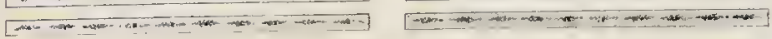

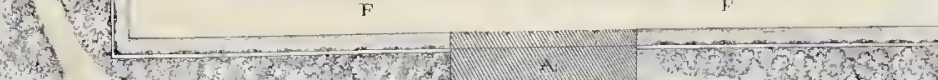
(n)

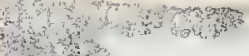

in 3 (.)

\section{sect}

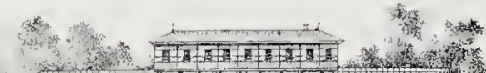

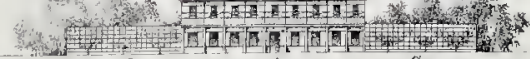

\section{A.}

$$
\text { by }
$$

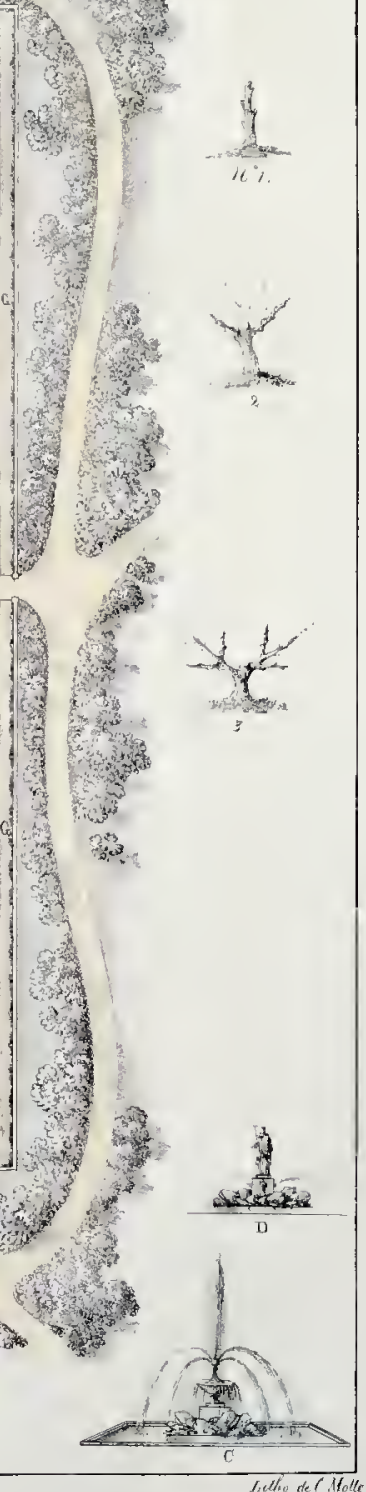

Thoun t) 


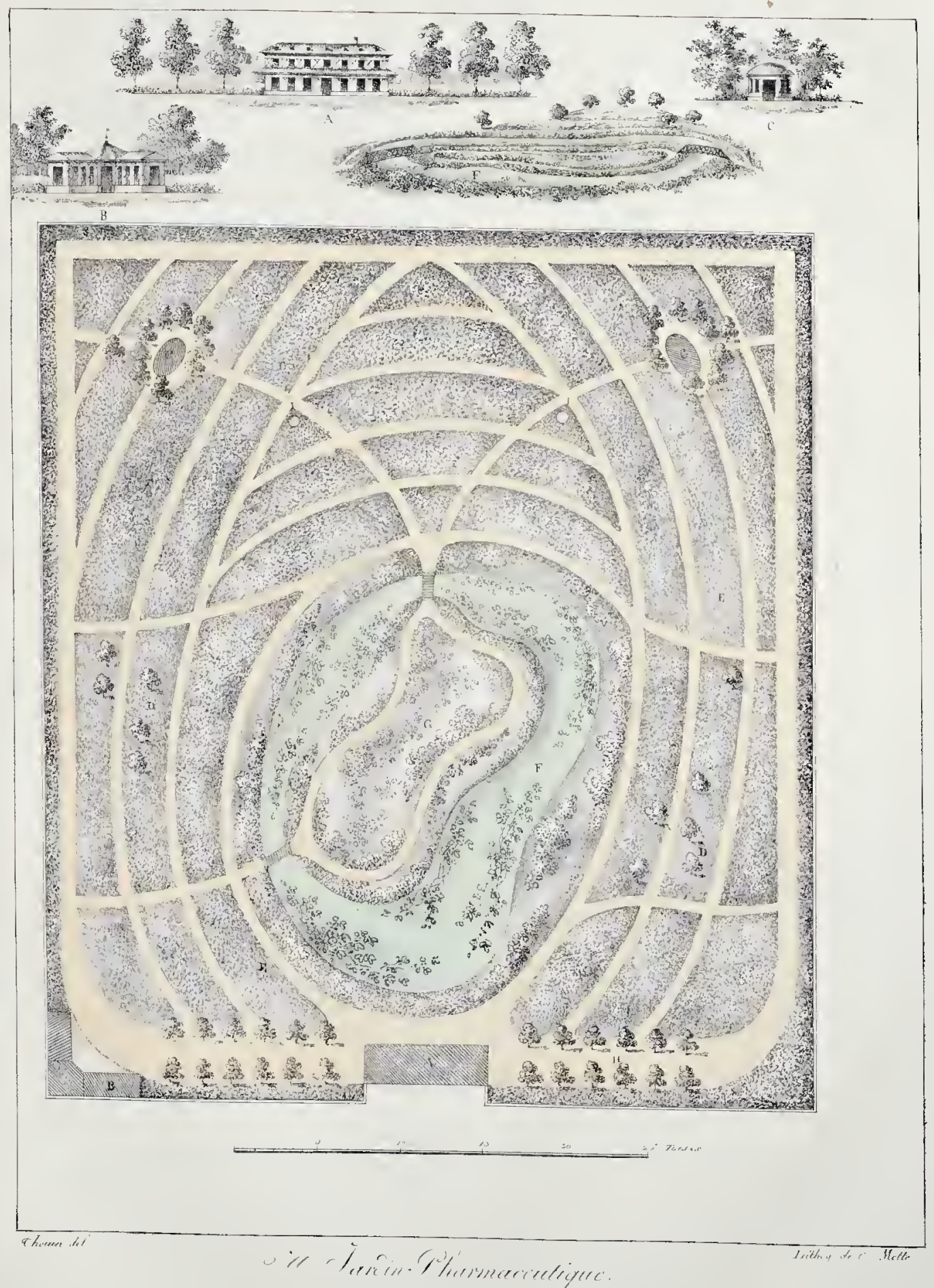




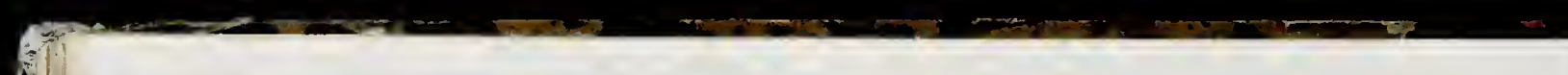



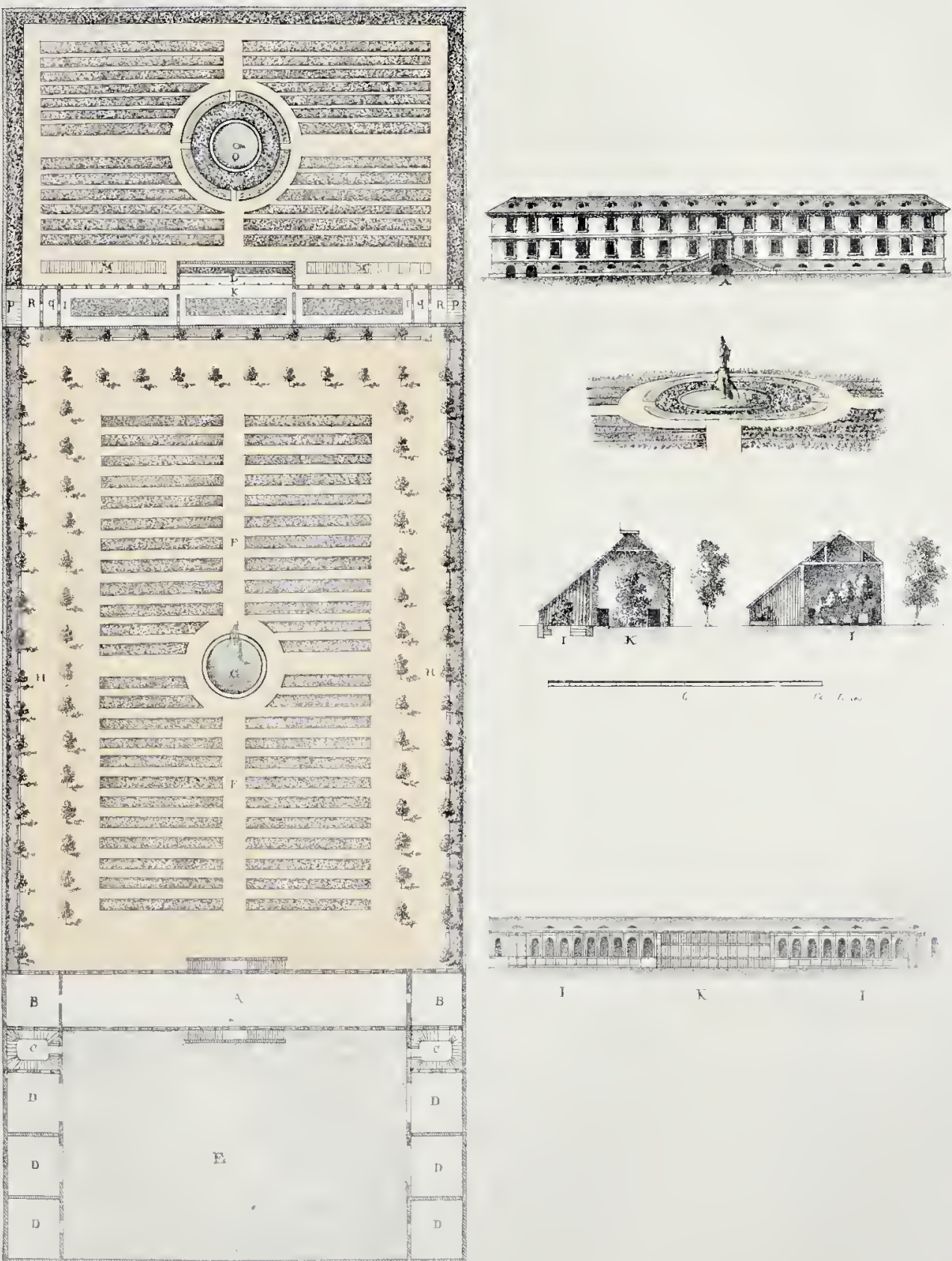


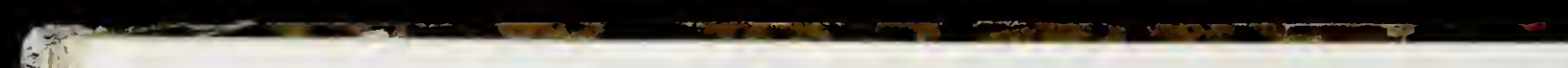

6.

. 


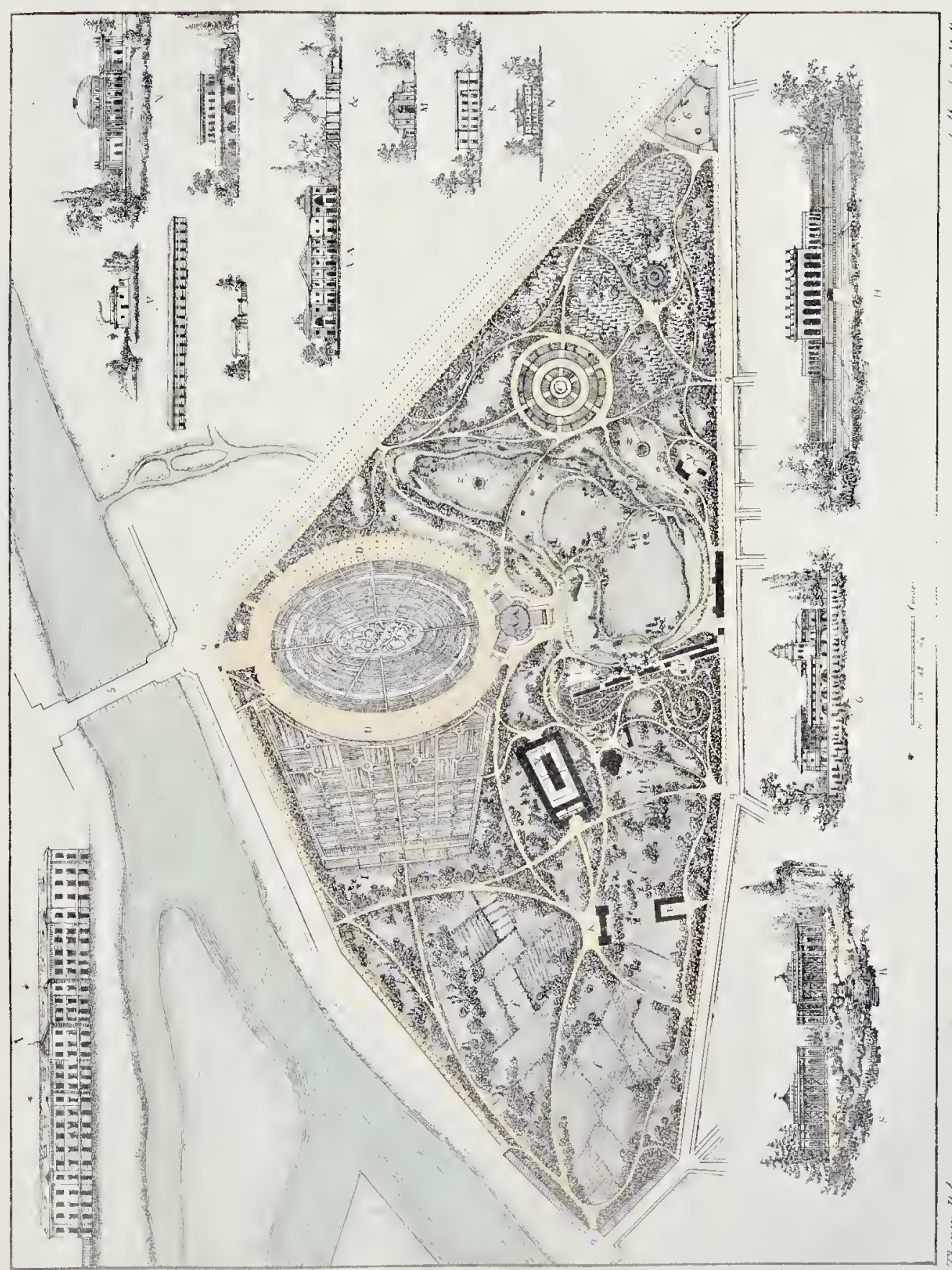




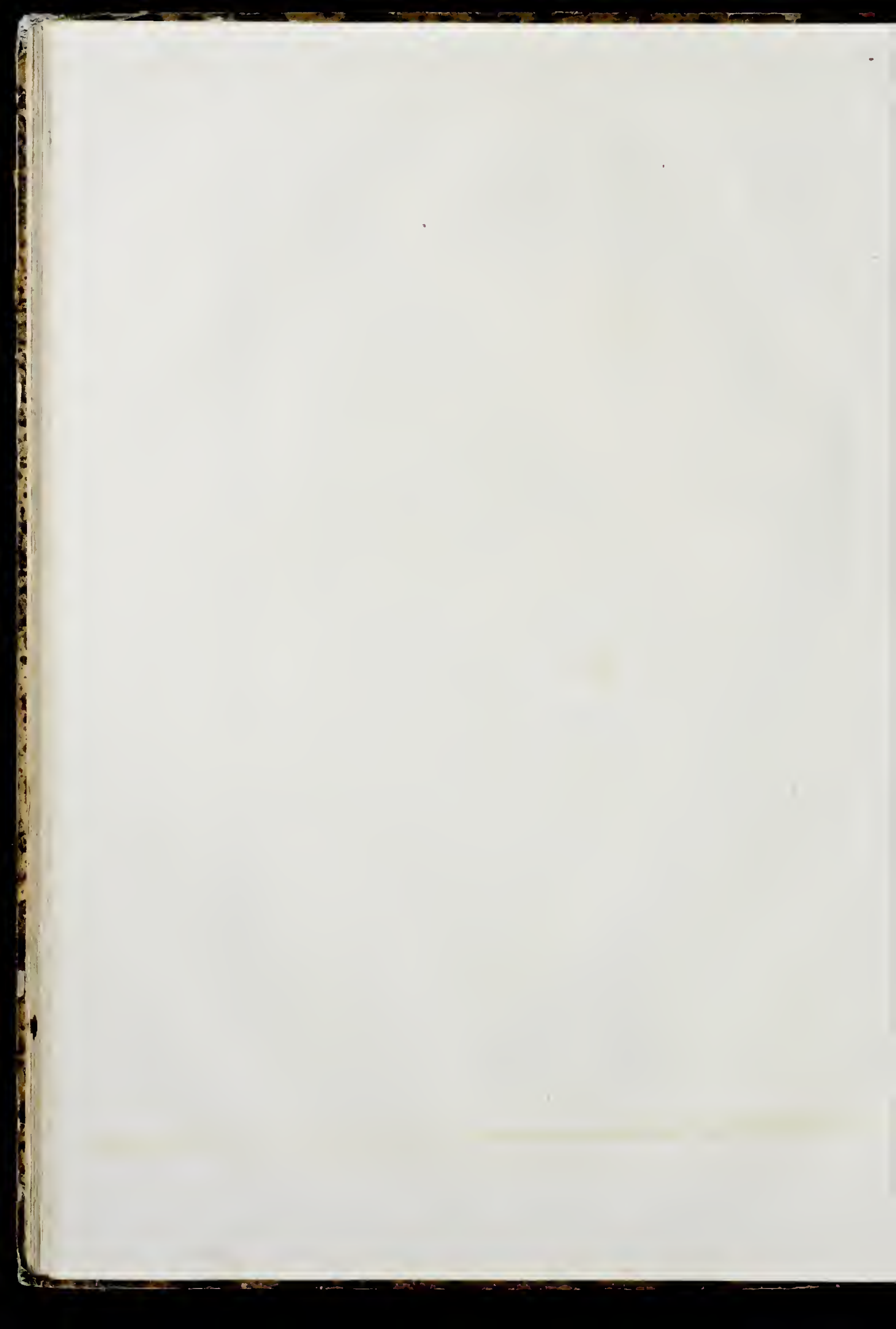




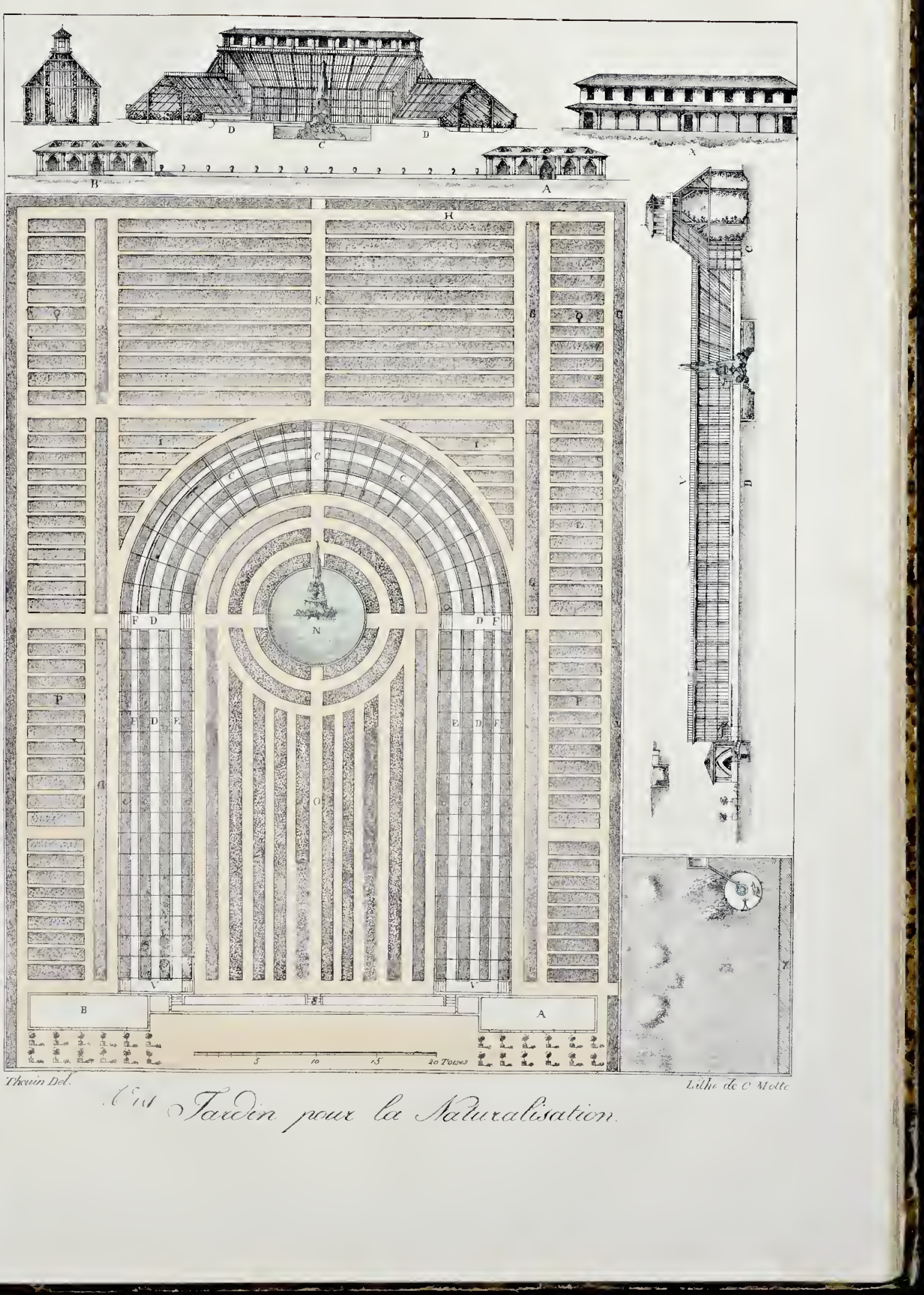




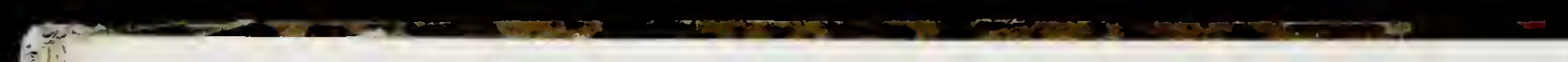

(1.). 

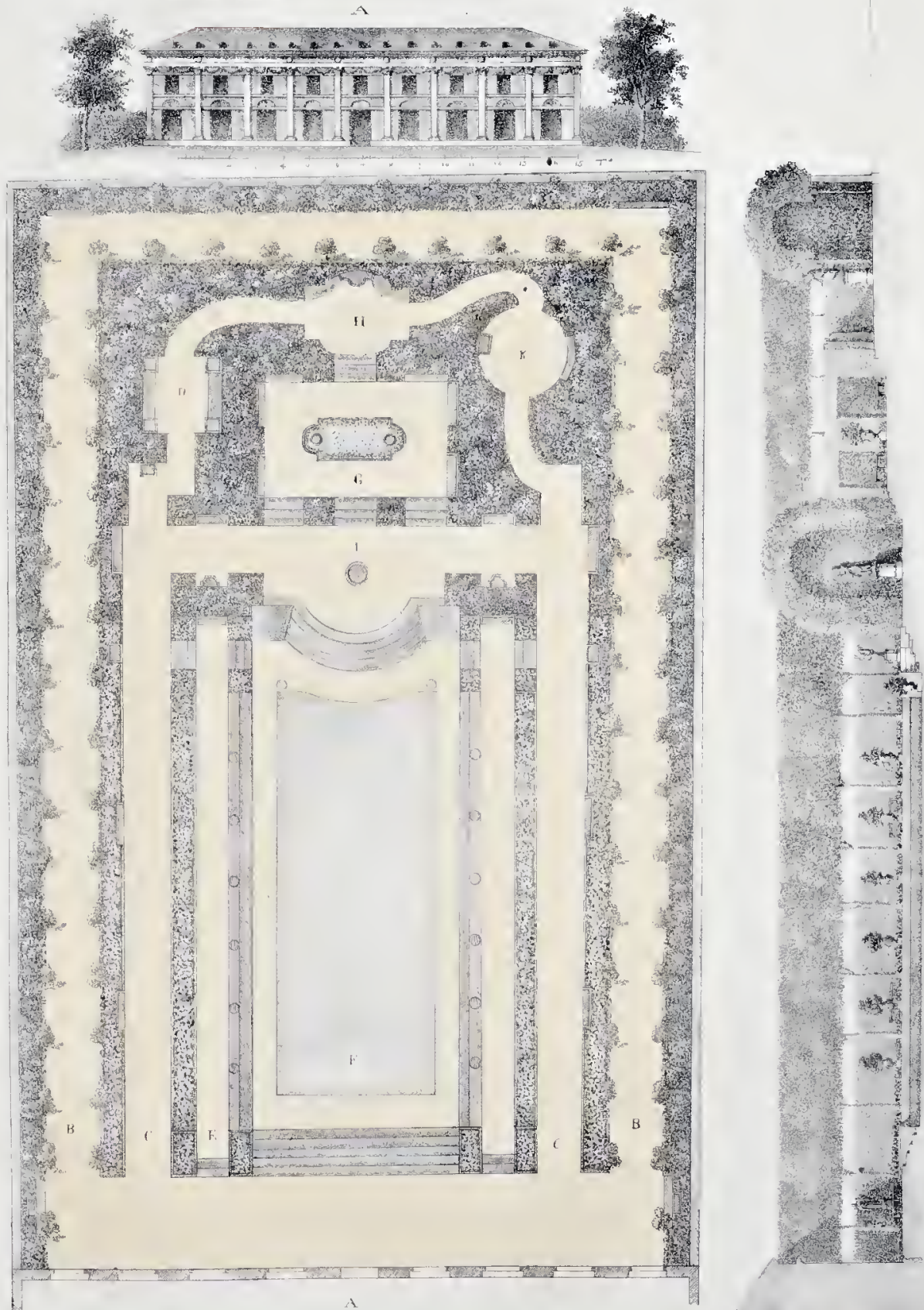

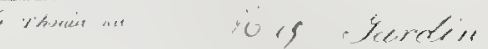




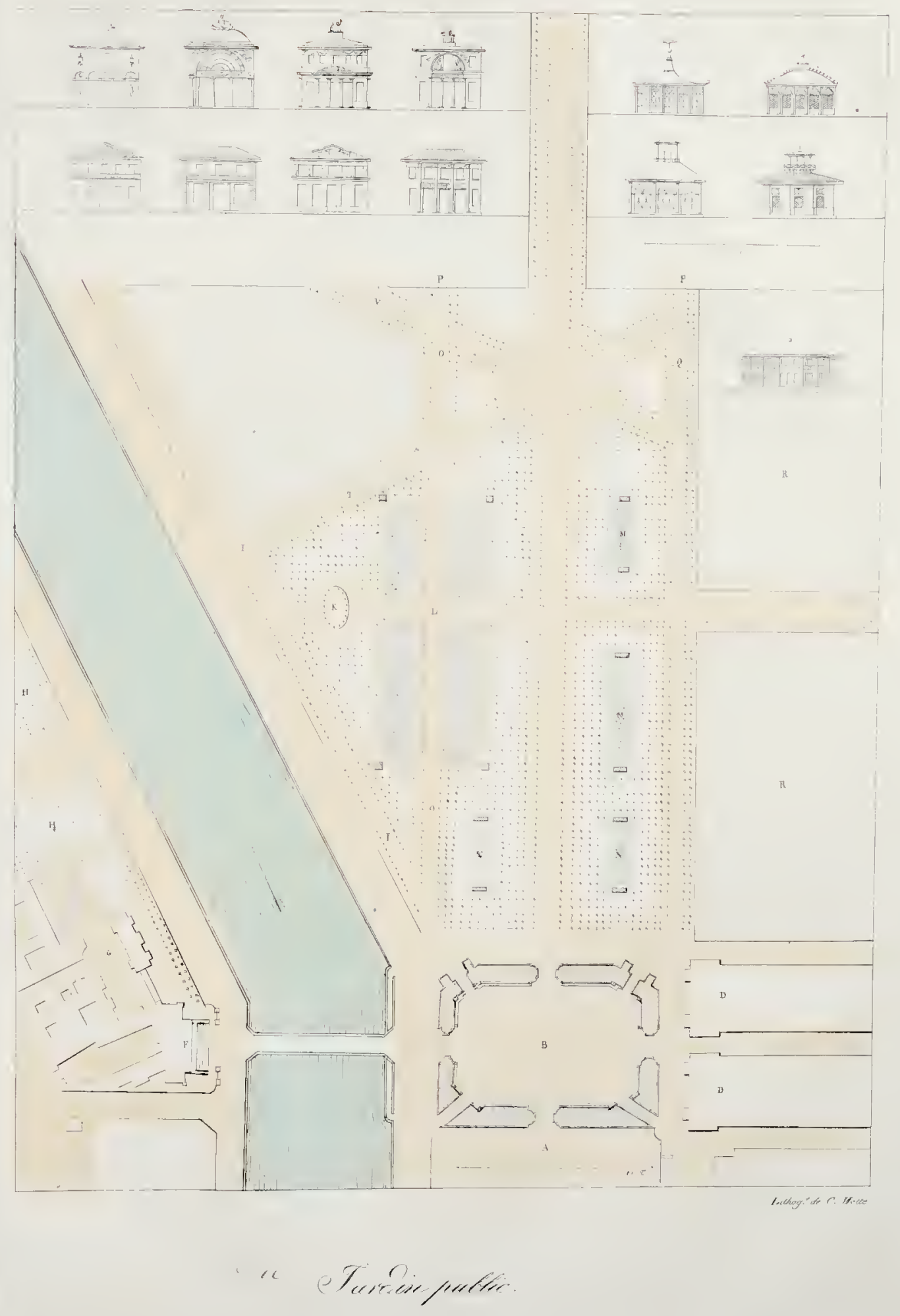




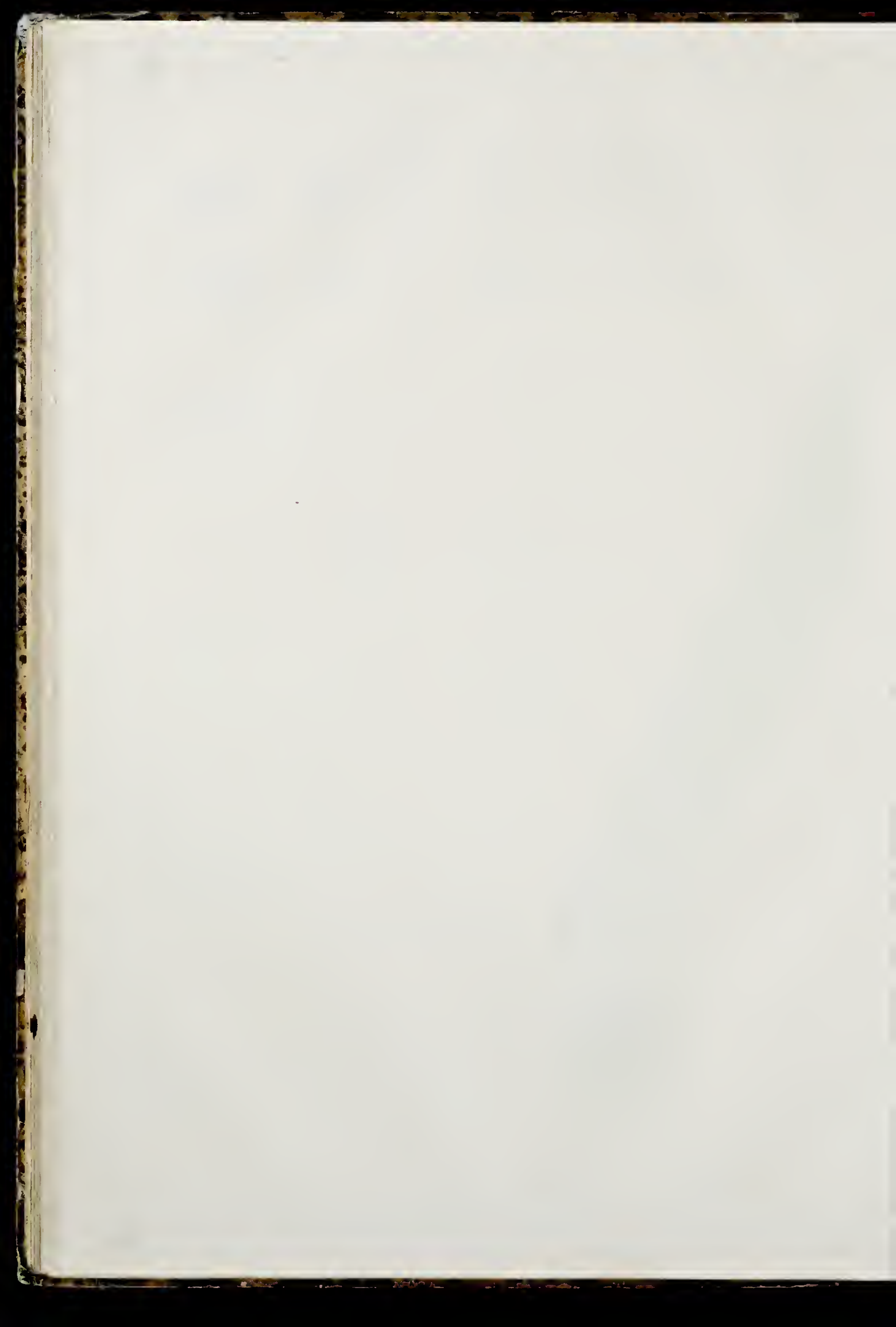




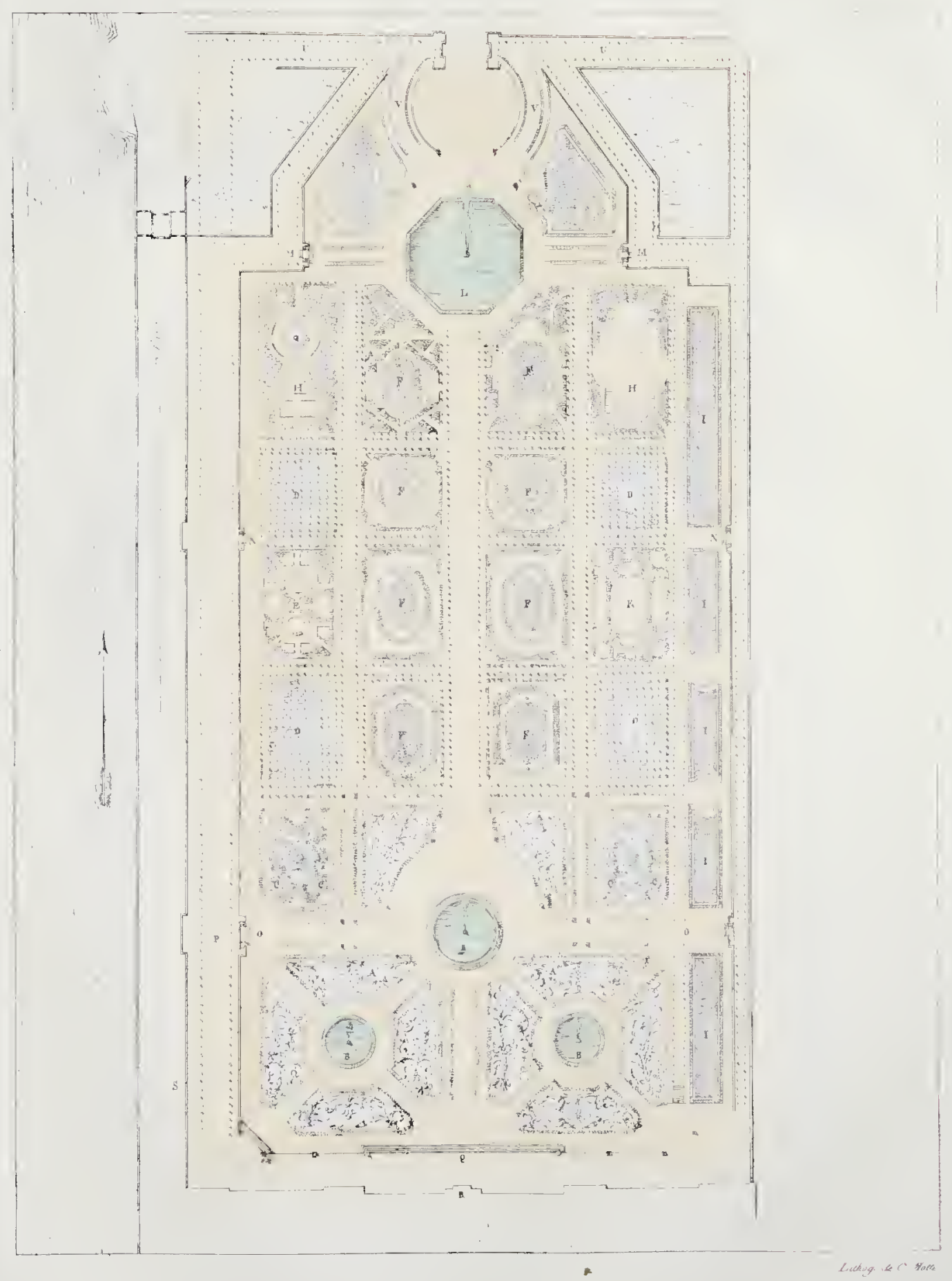

Sarerne If Latast.

keo Thuleriez 


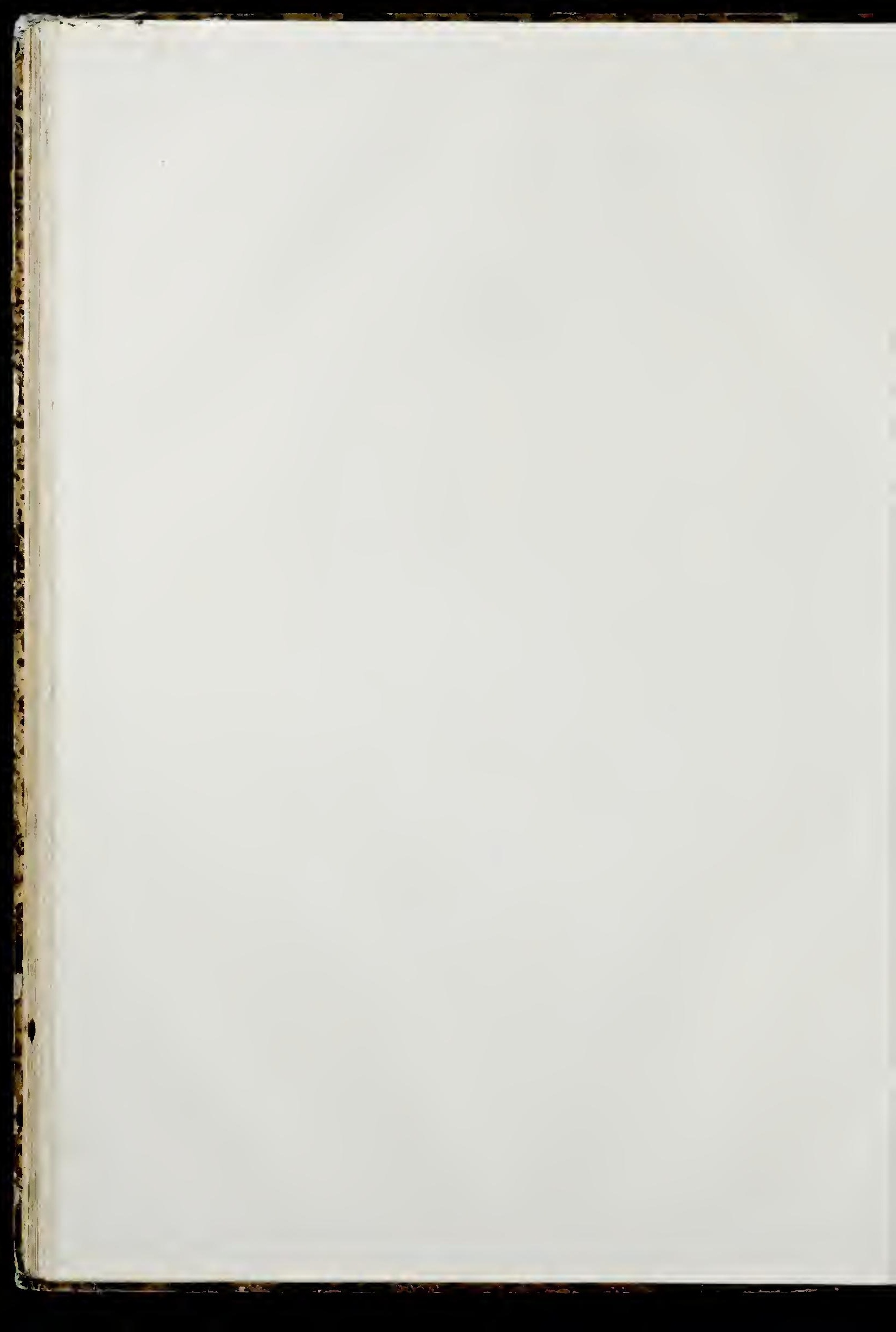




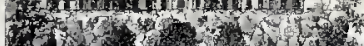

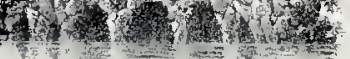

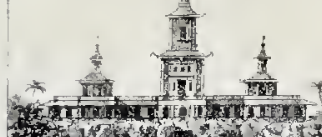

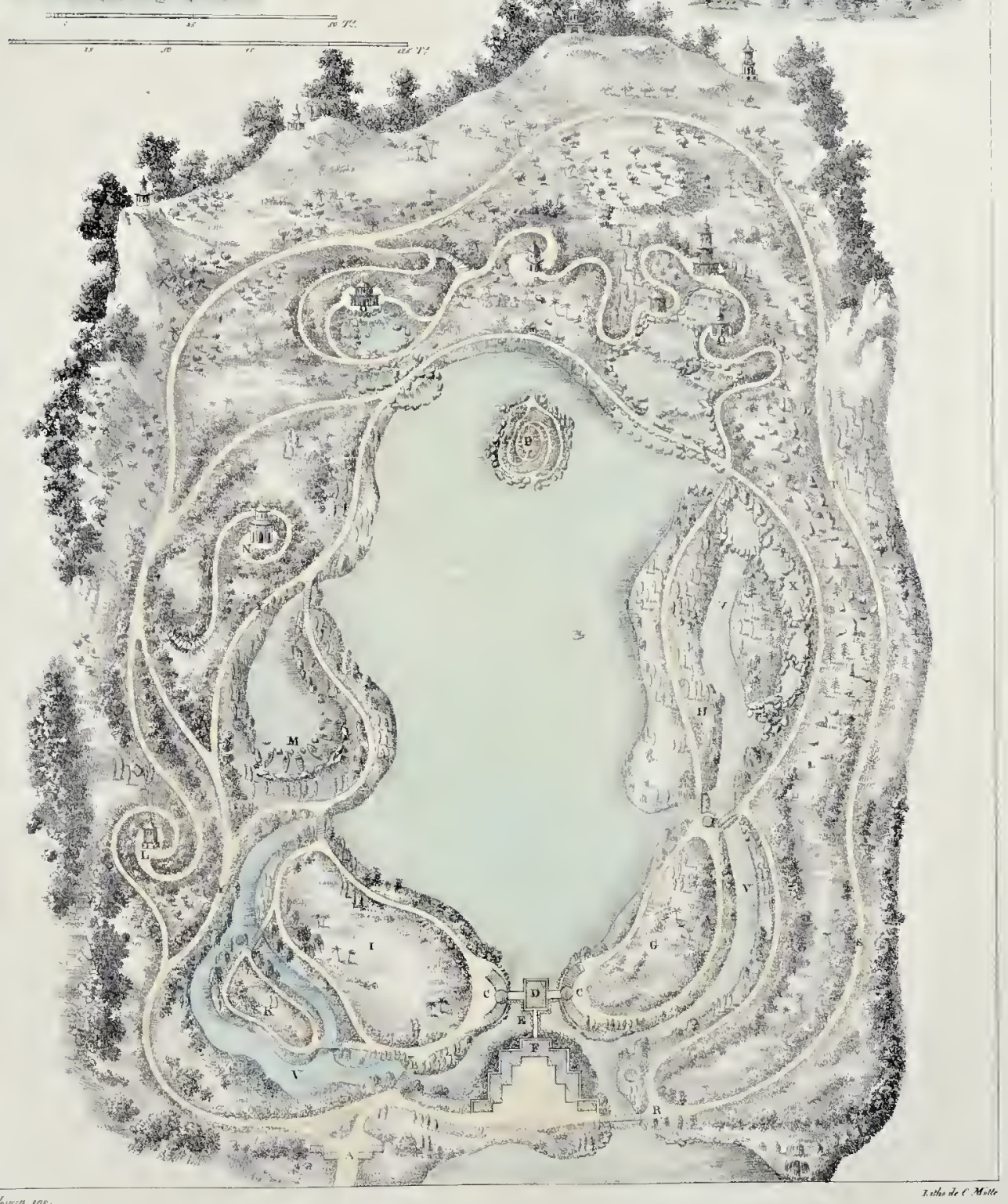




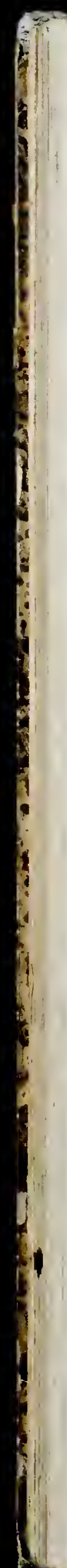




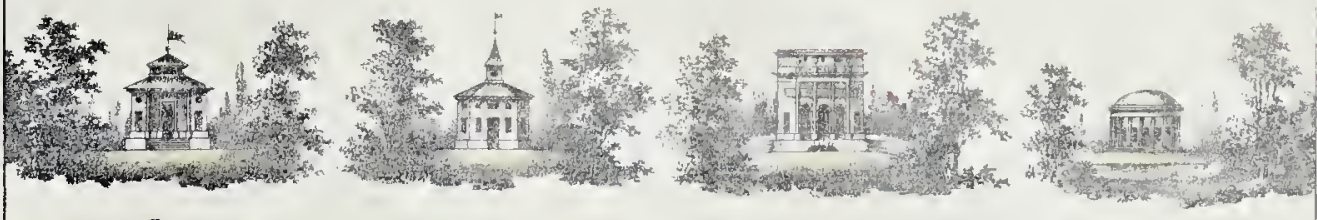
9

\section{$\mathrm{H}$}
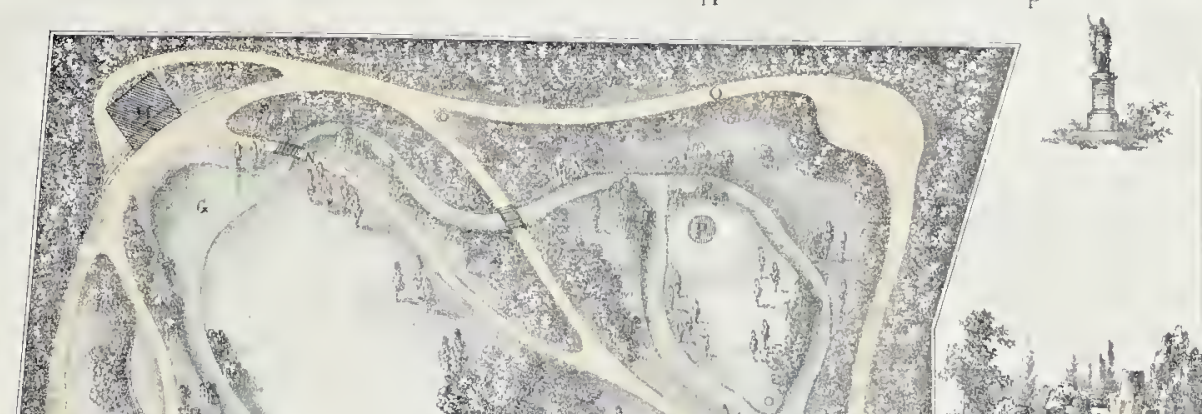


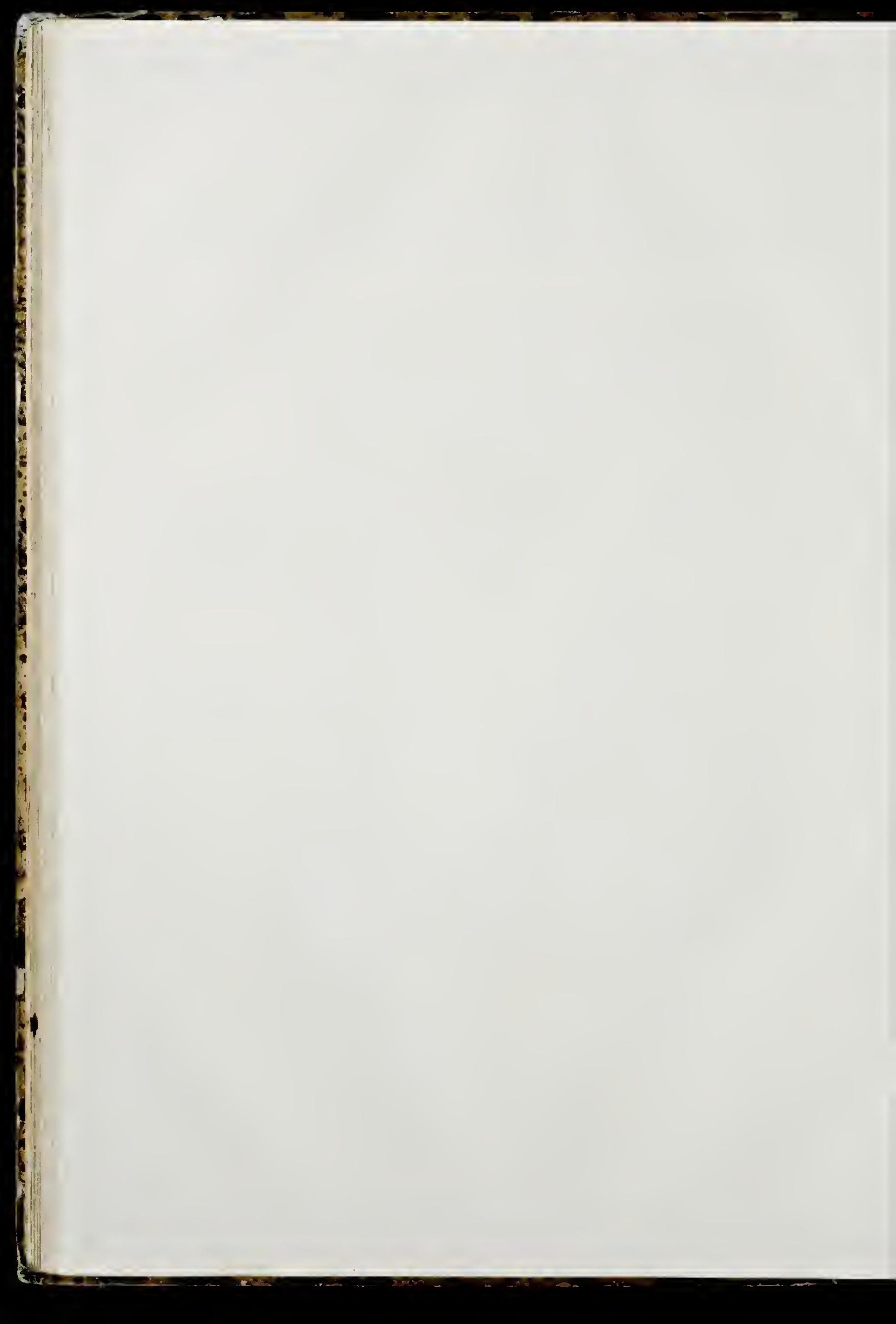




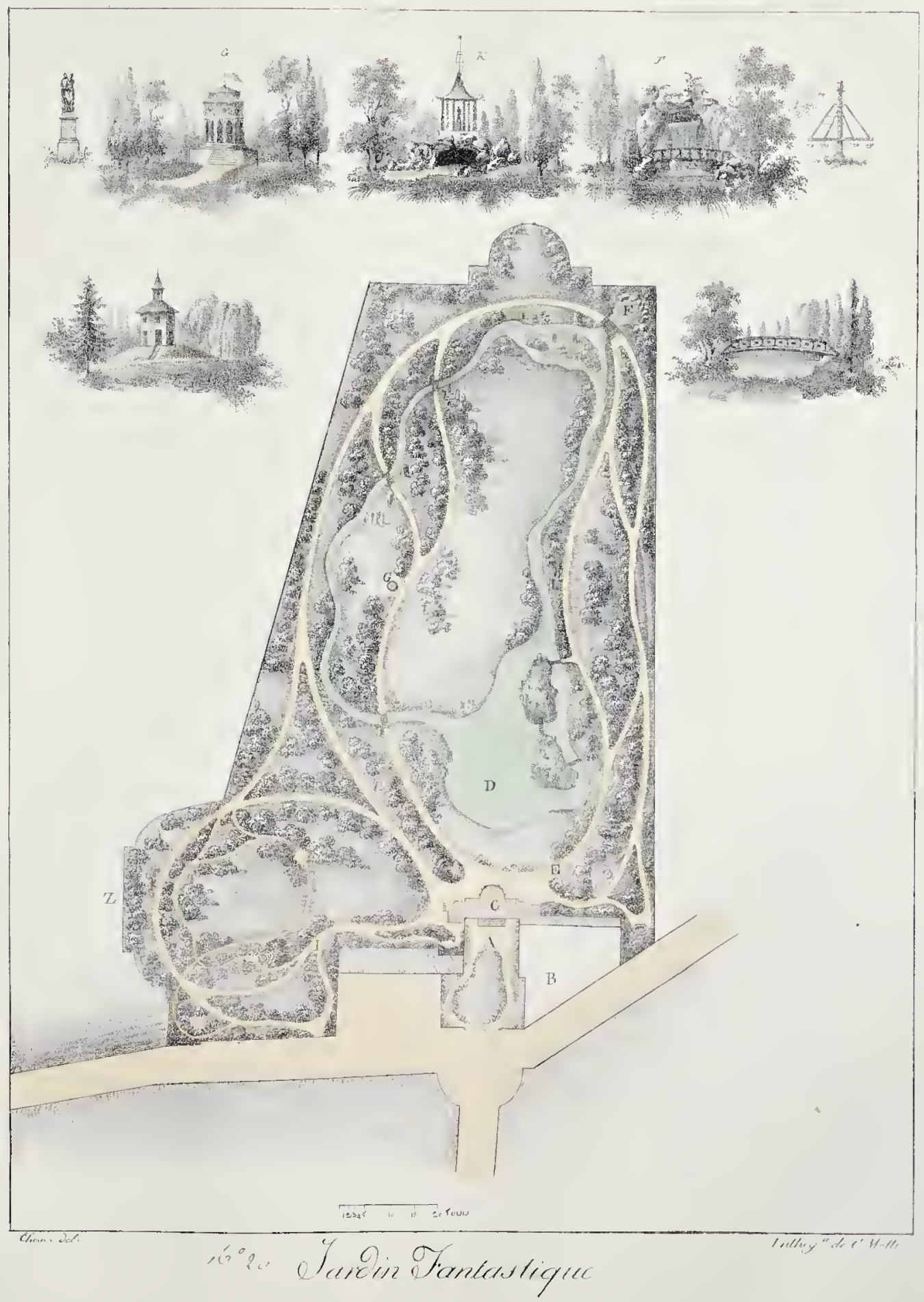




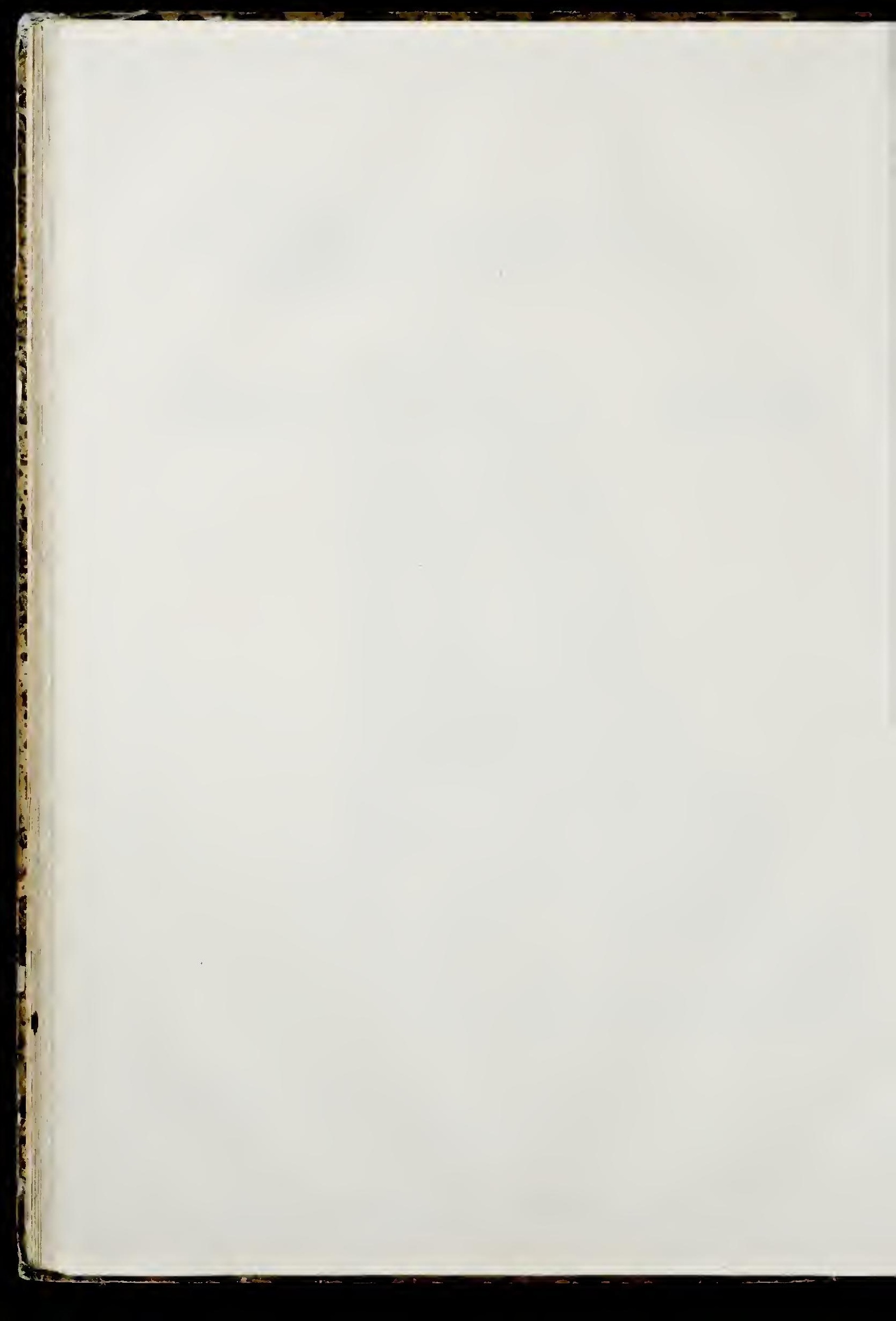




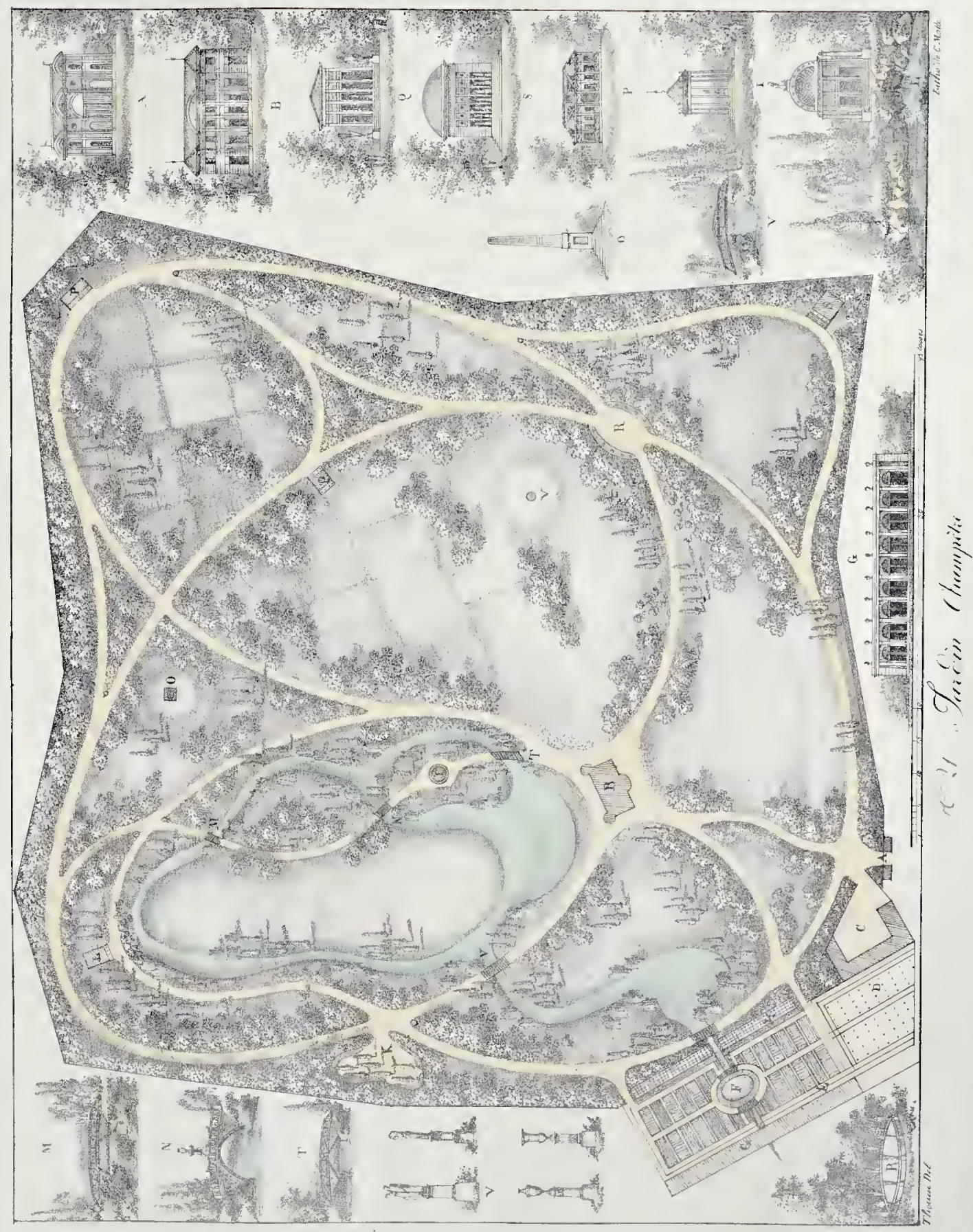




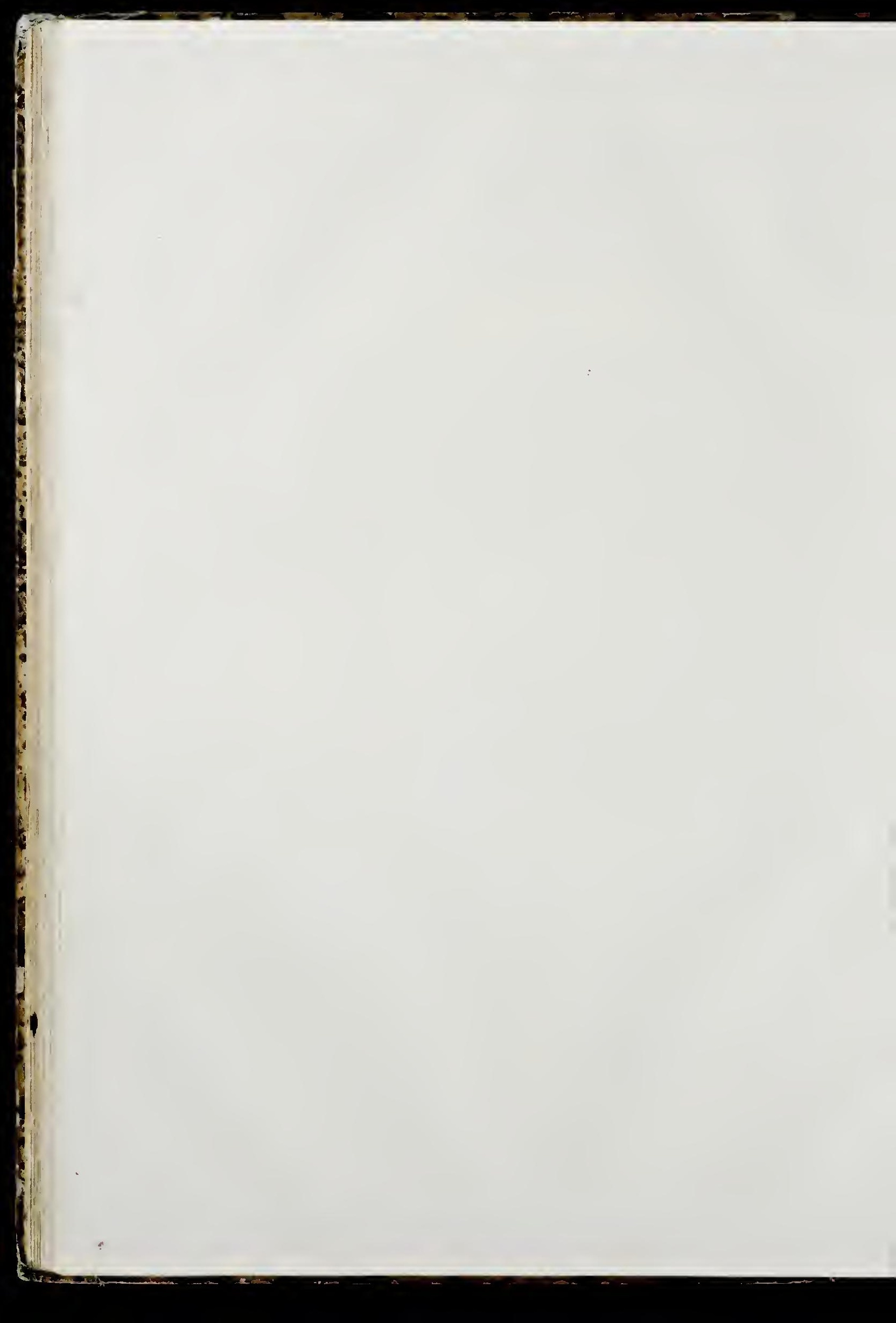




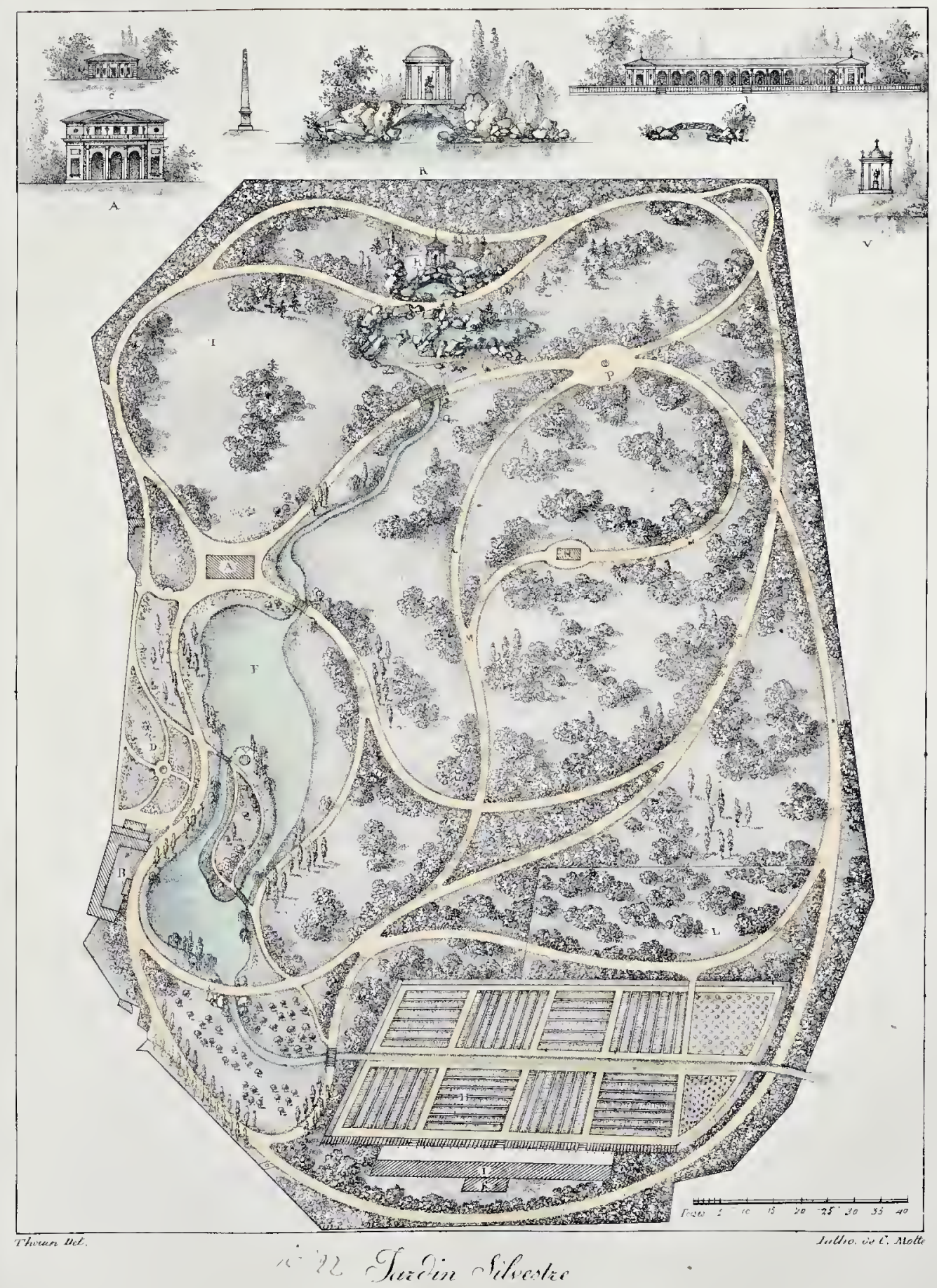




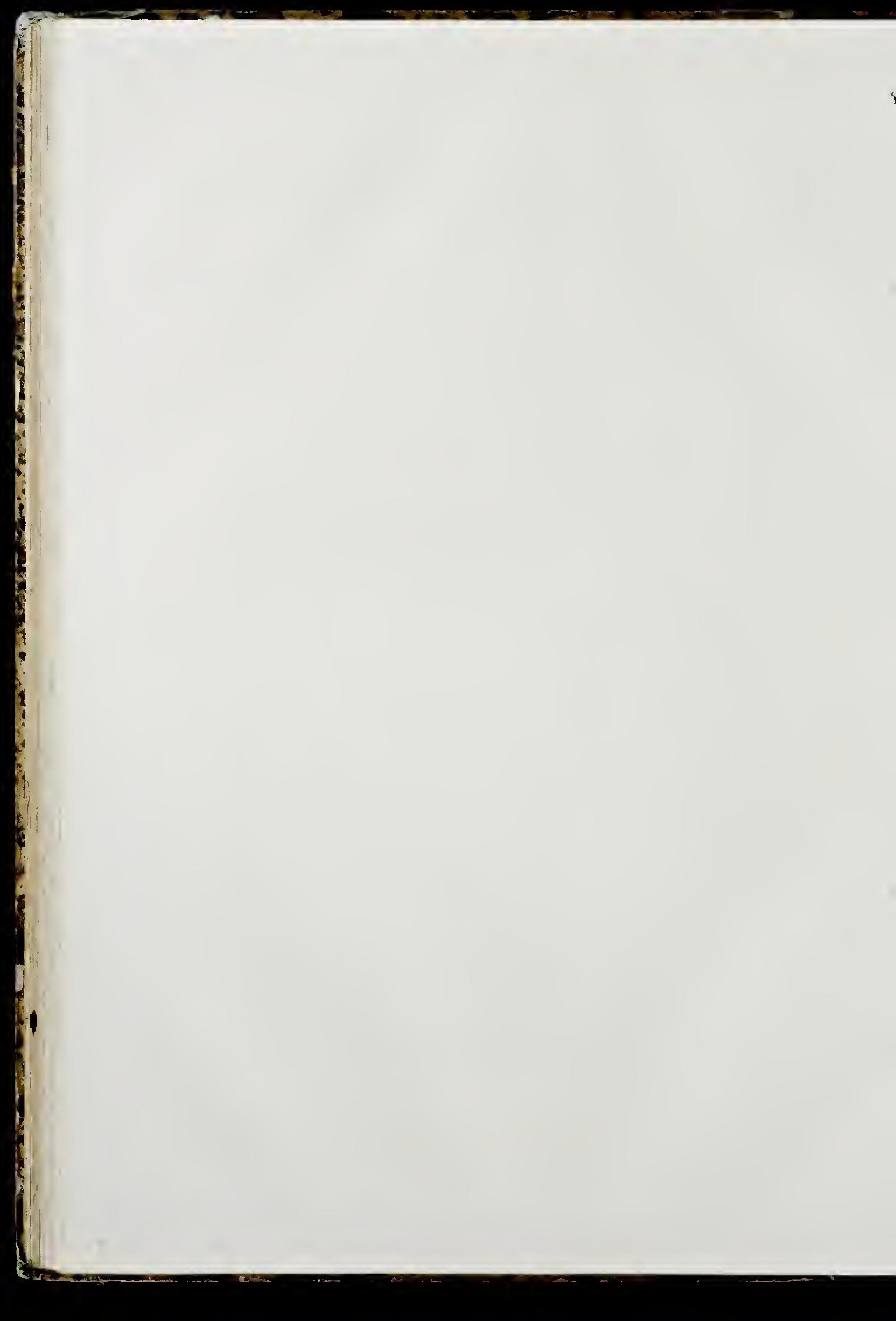




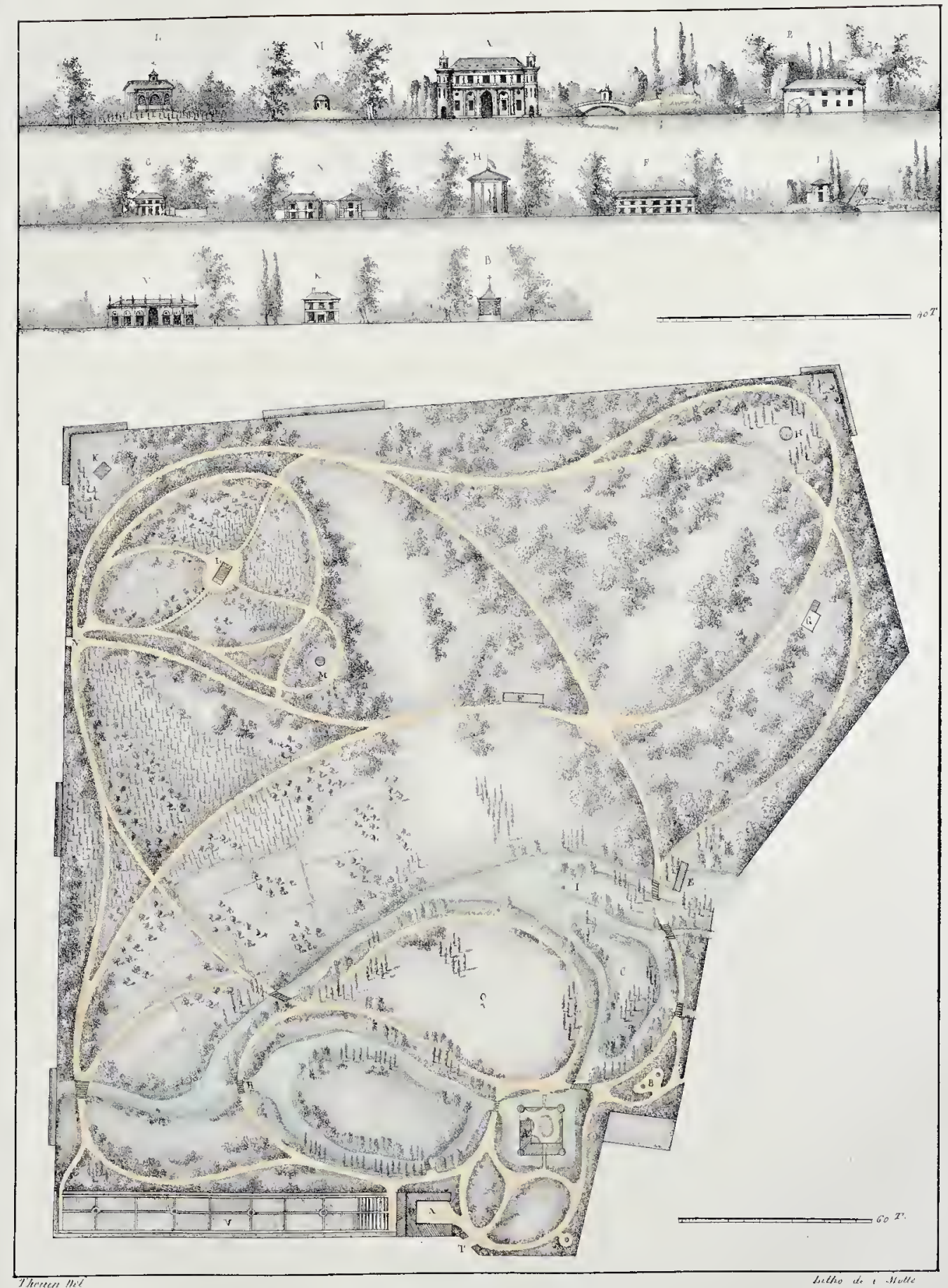




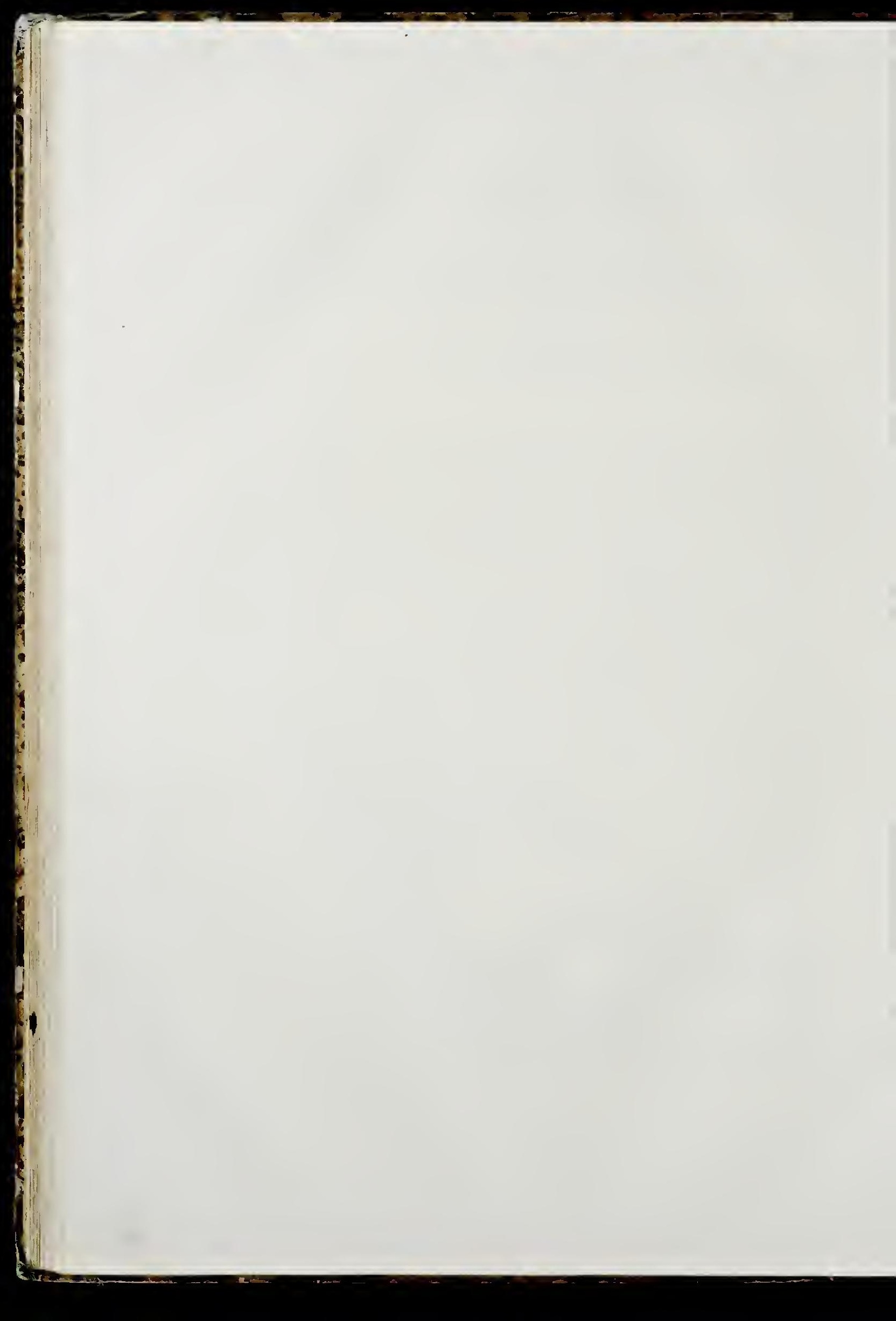




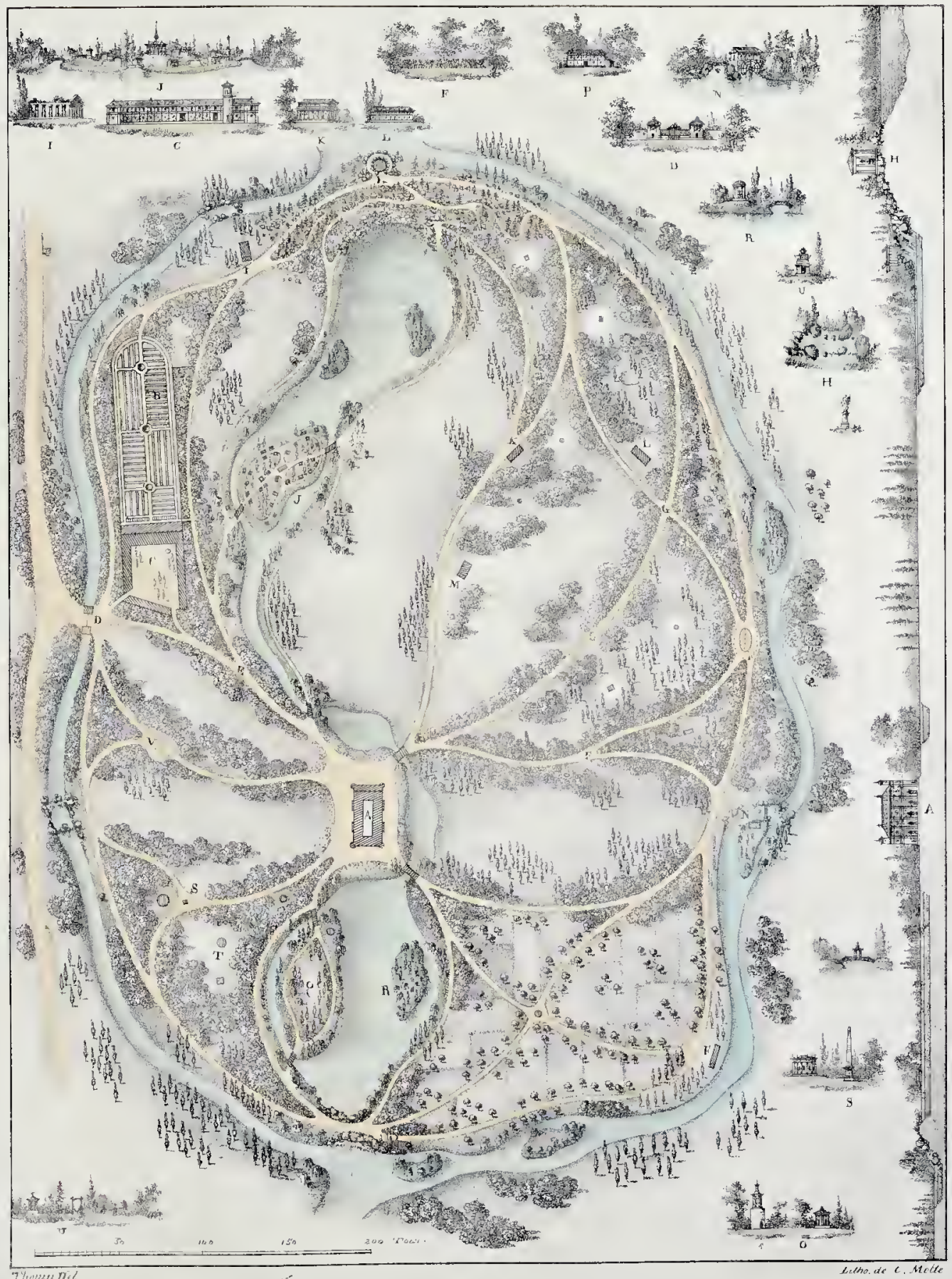




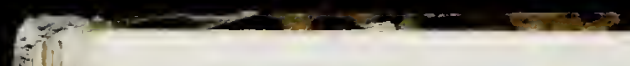

$-\infty-1+x-1$

t. 


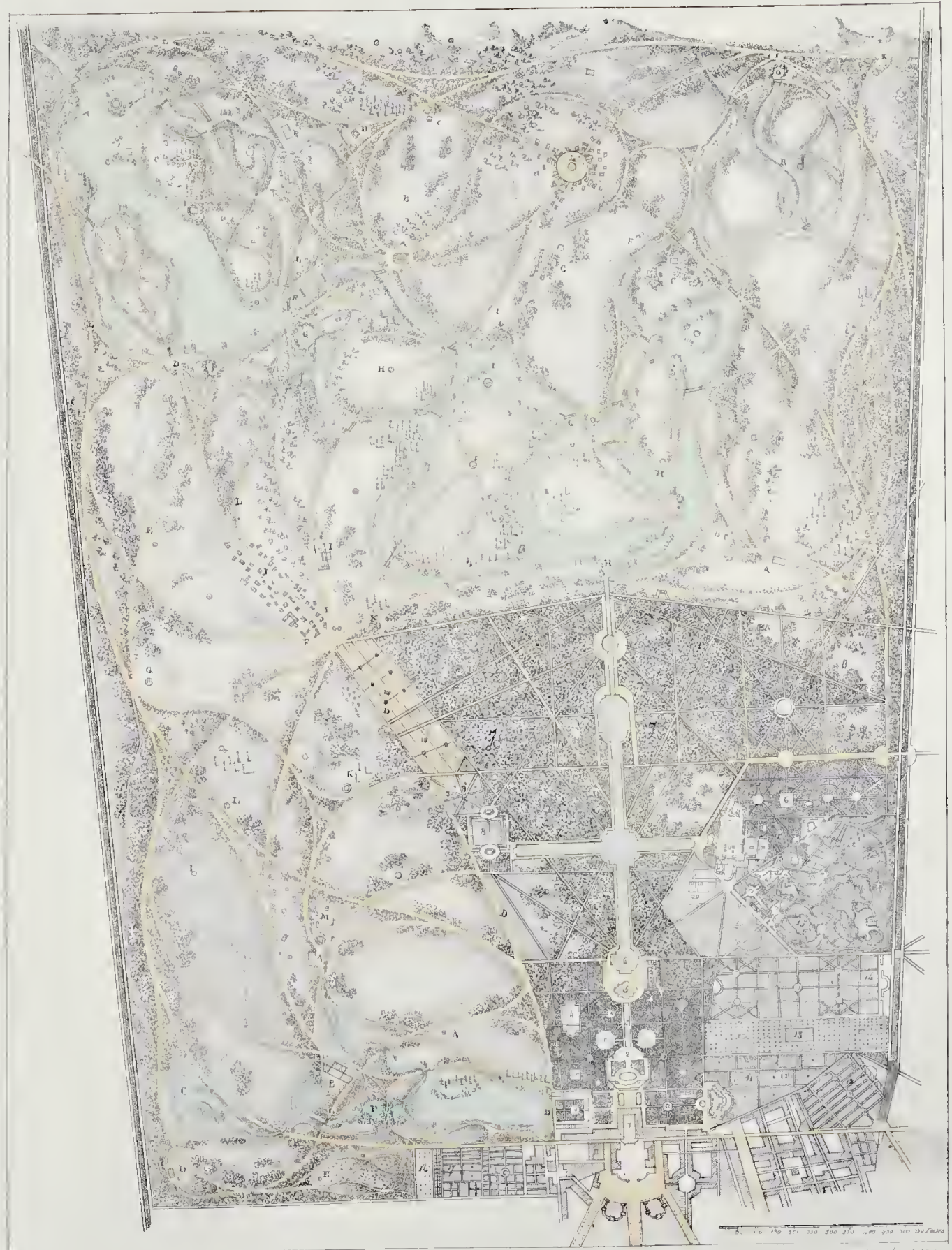

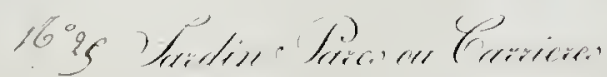




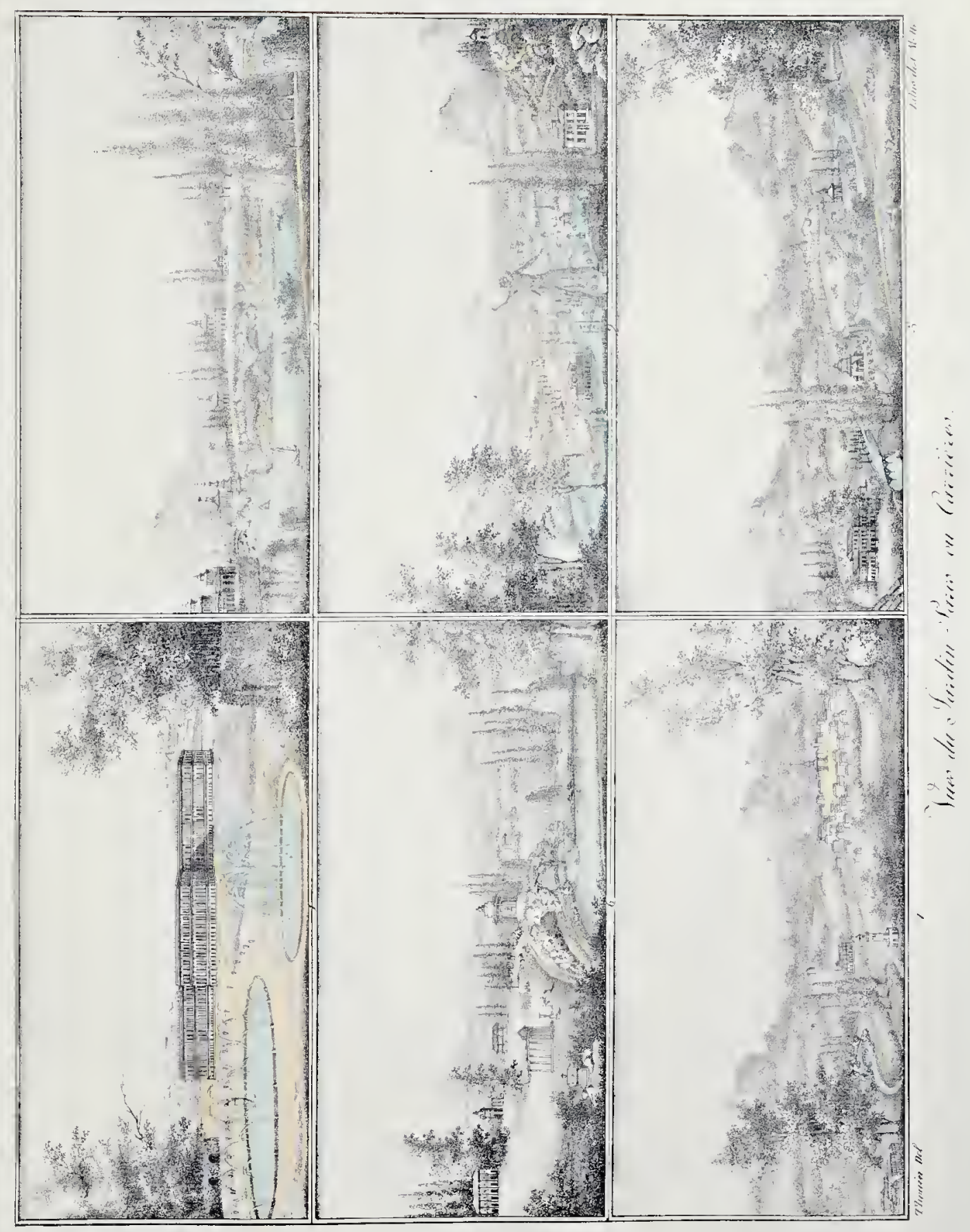




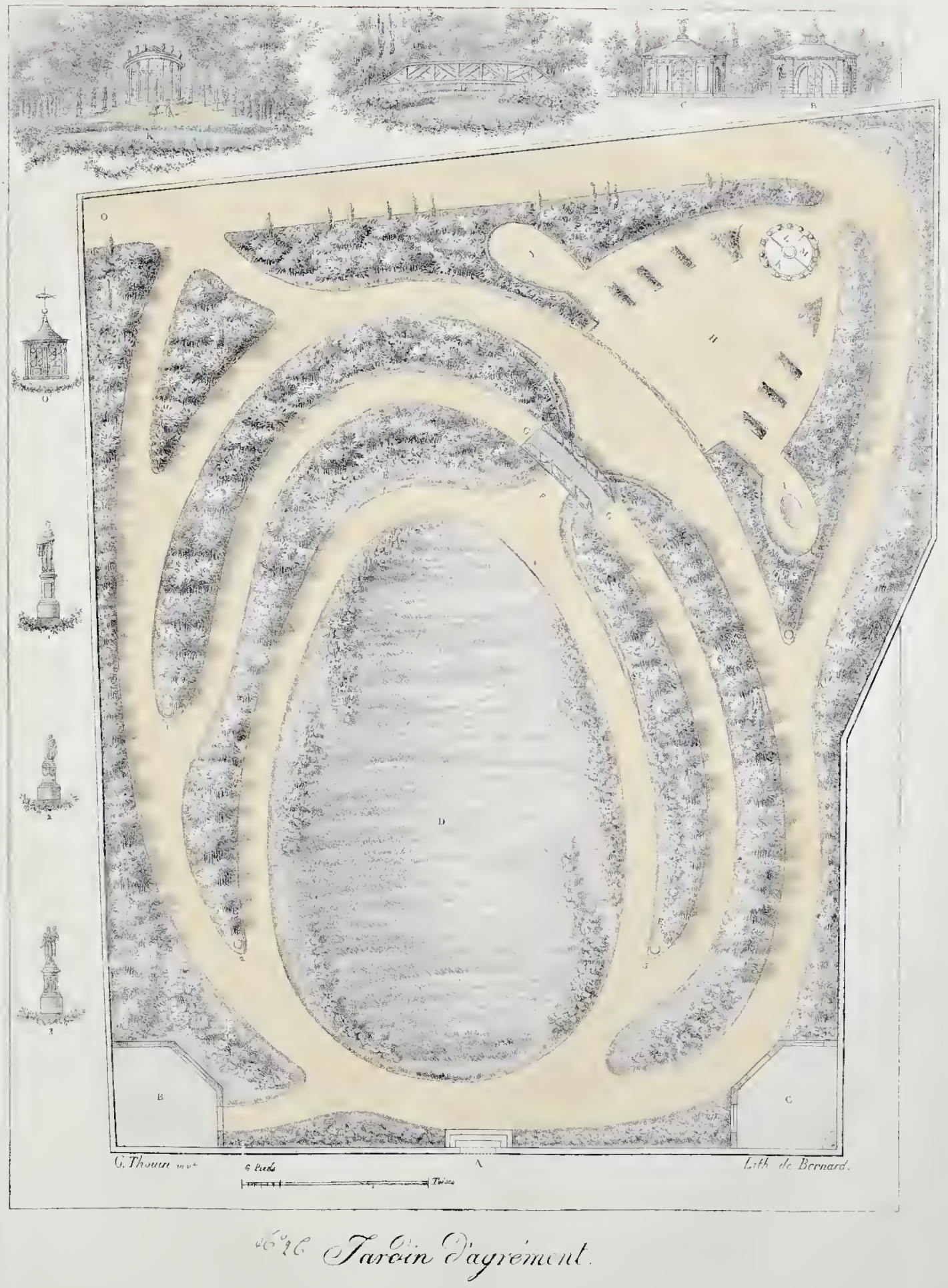




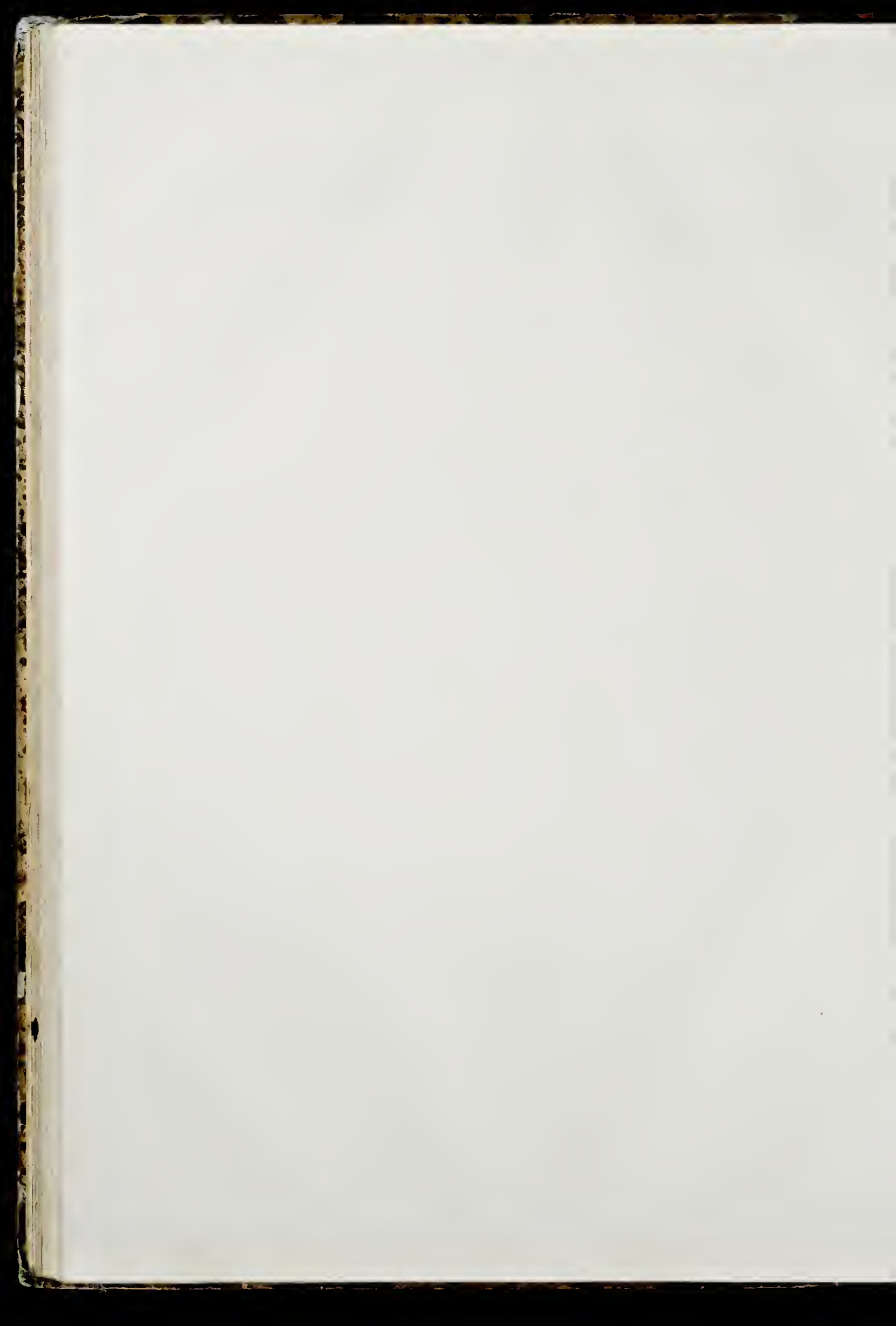




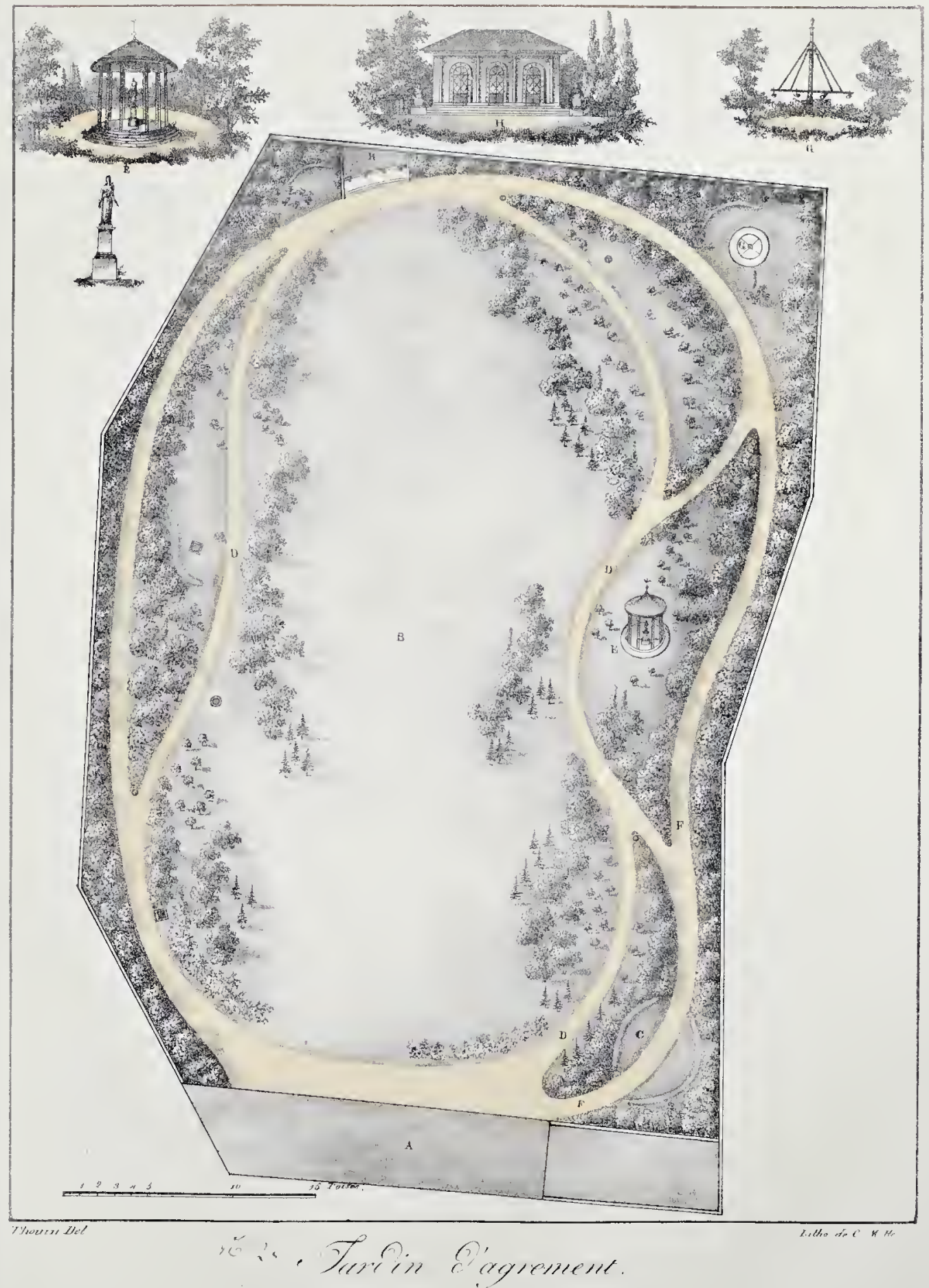




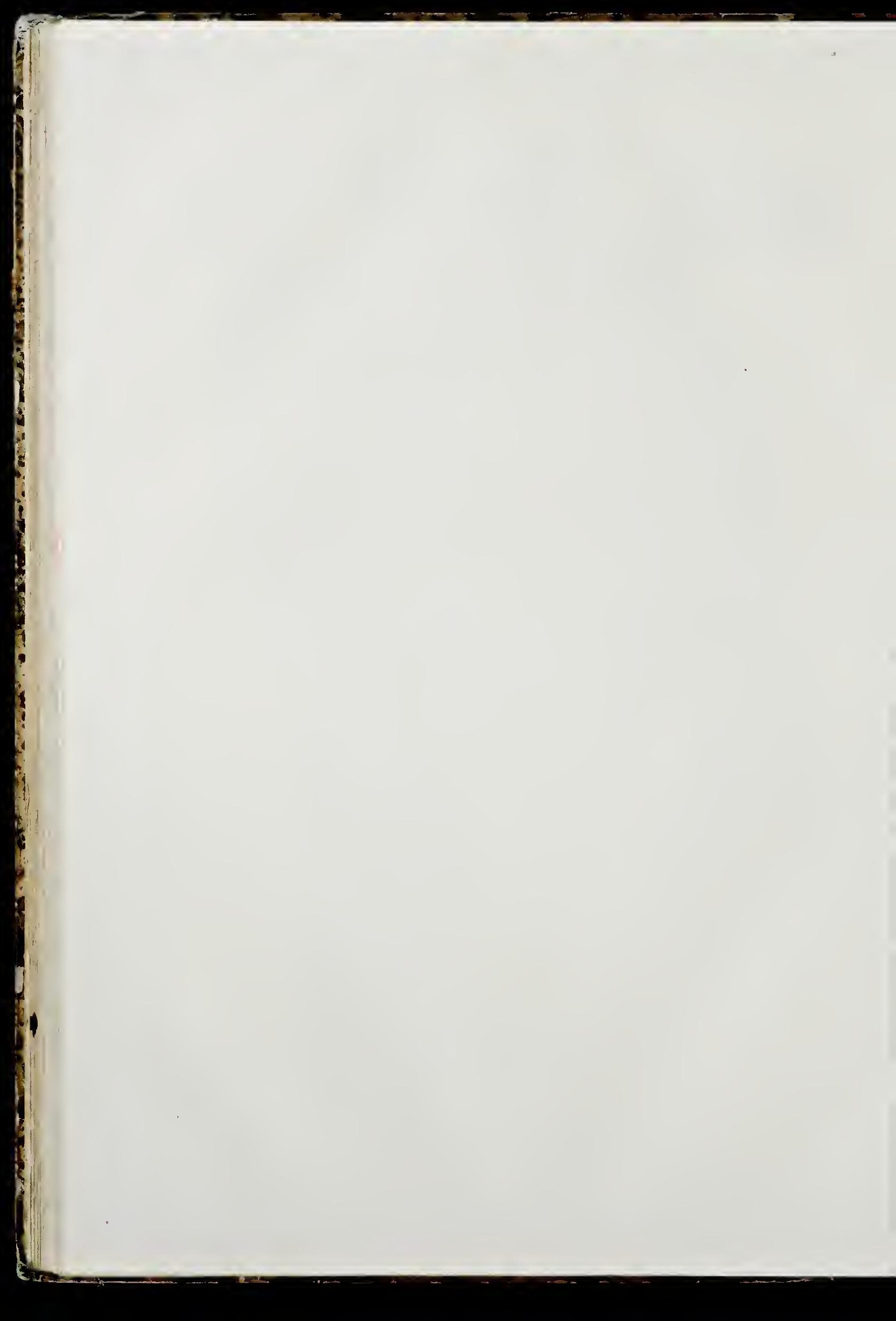



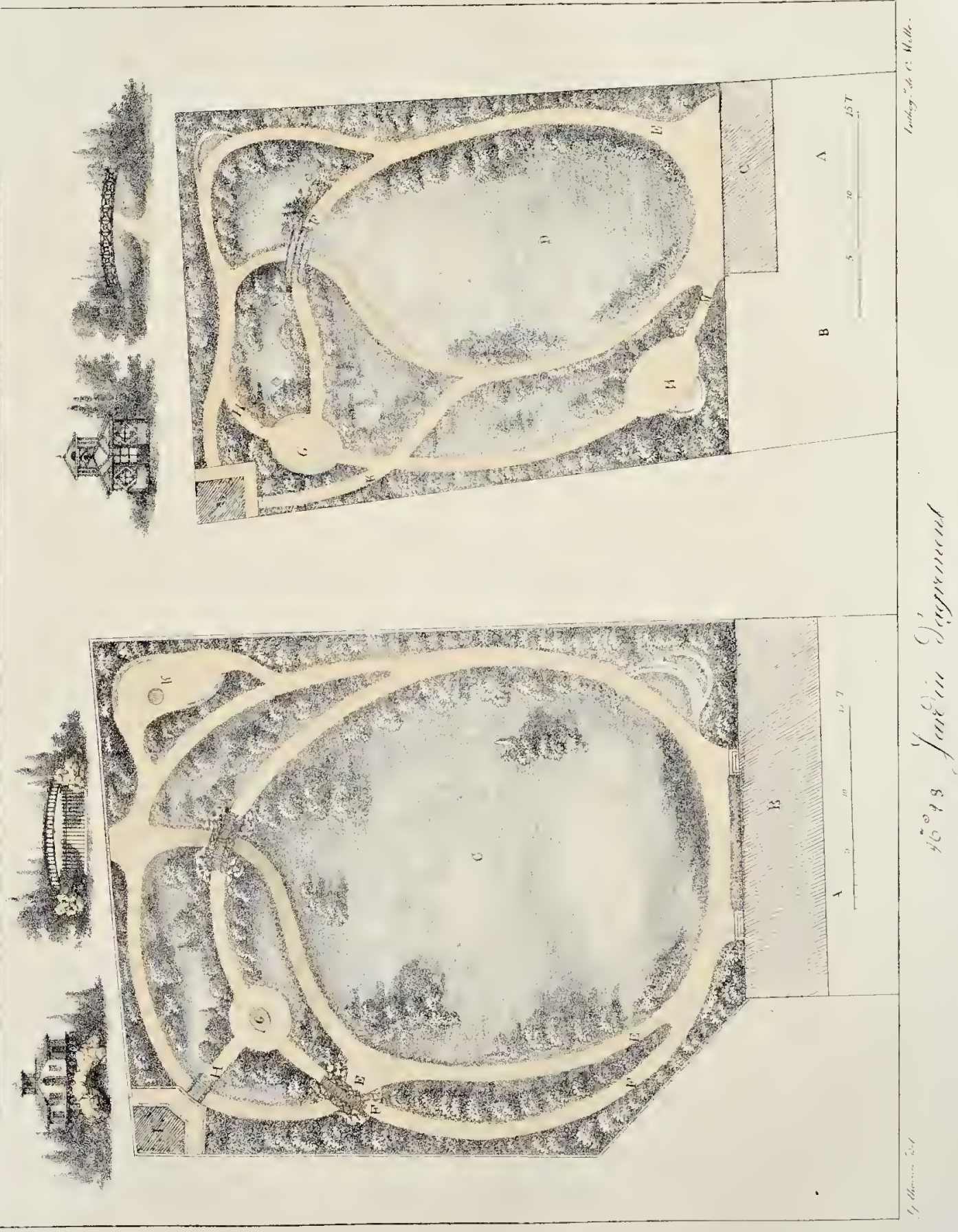


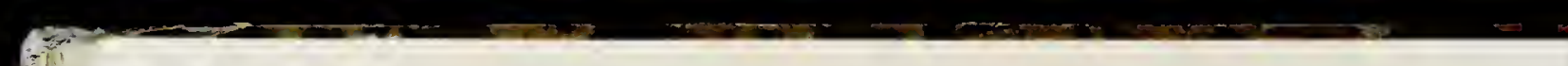




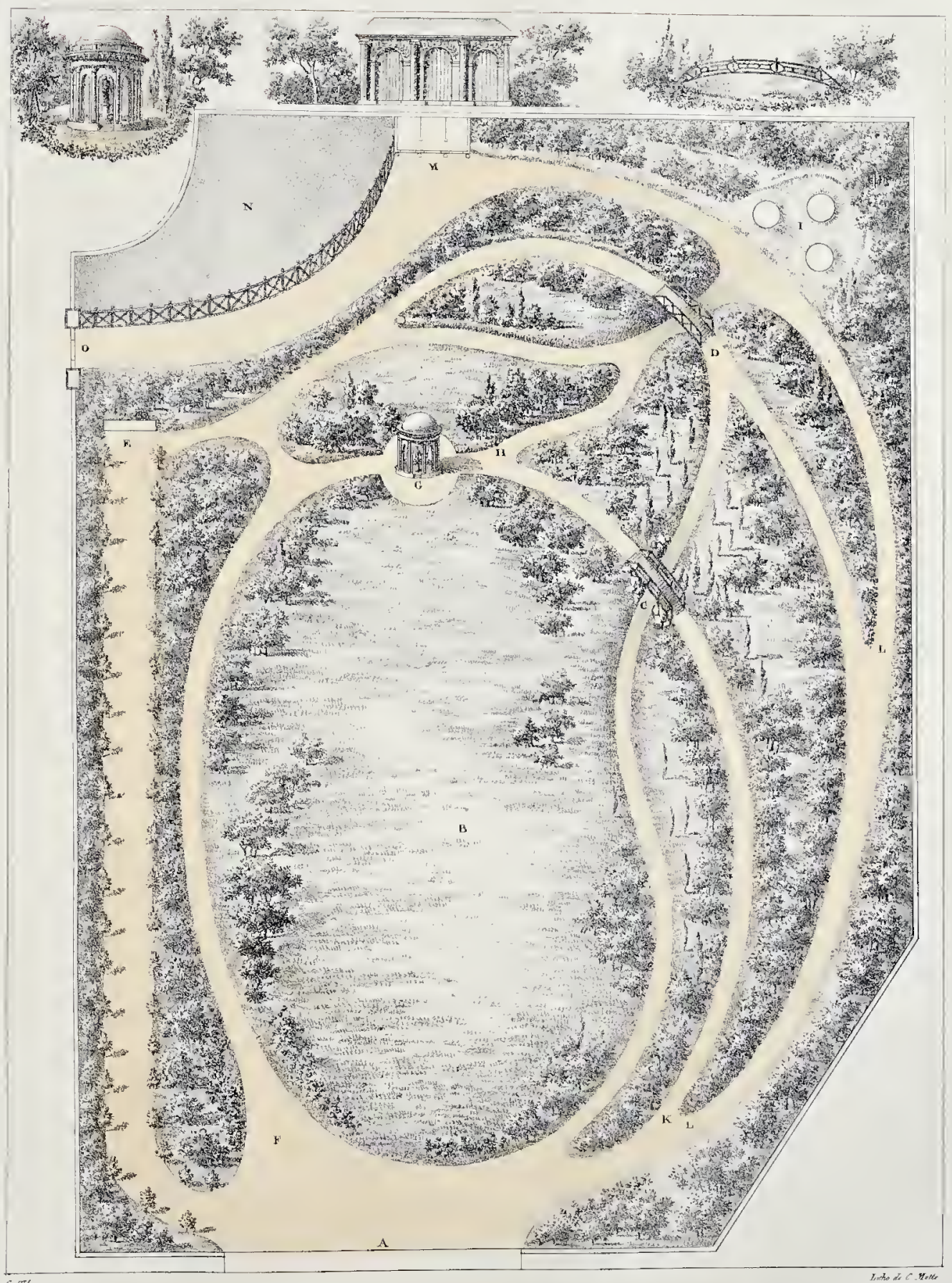




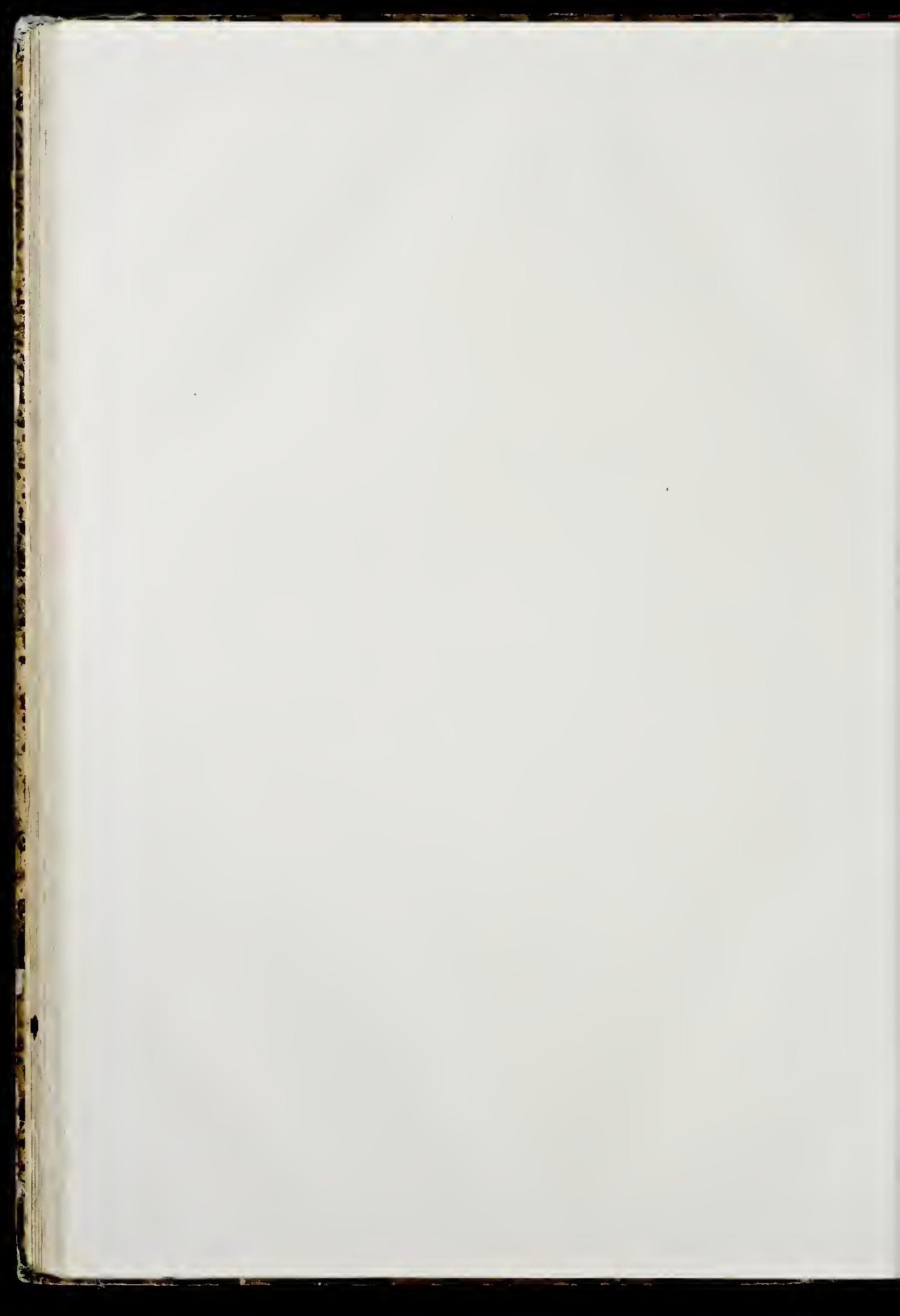




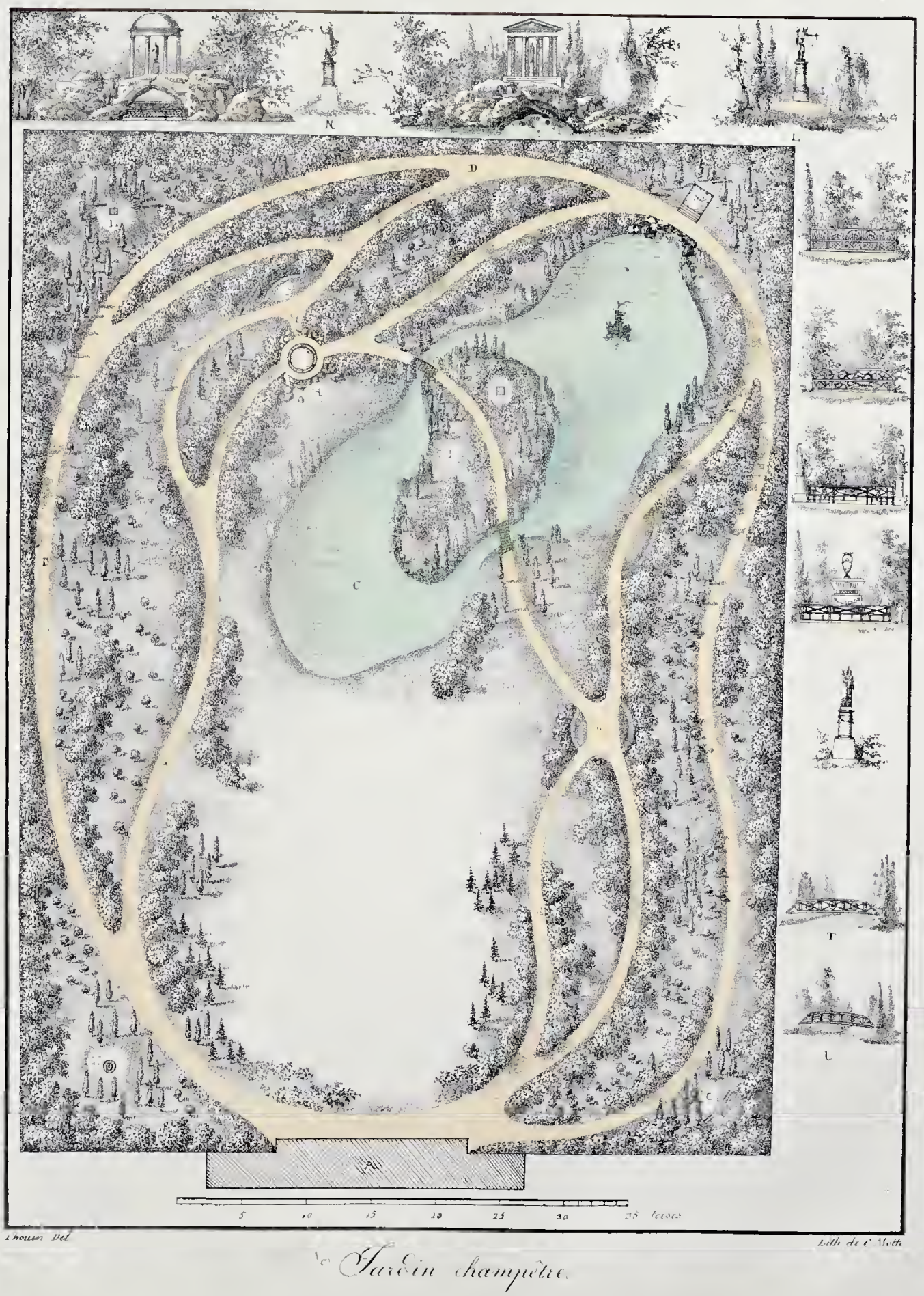




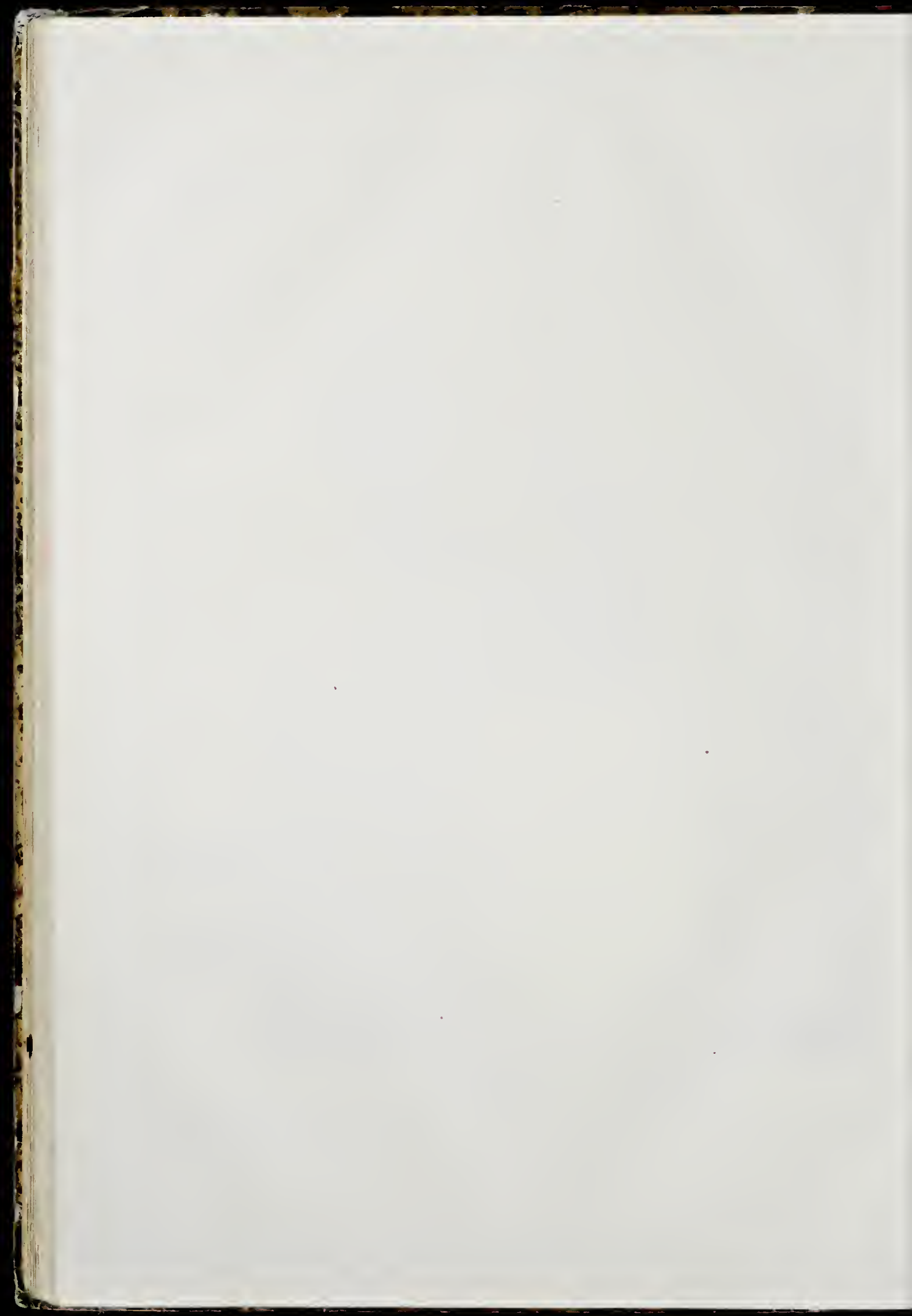




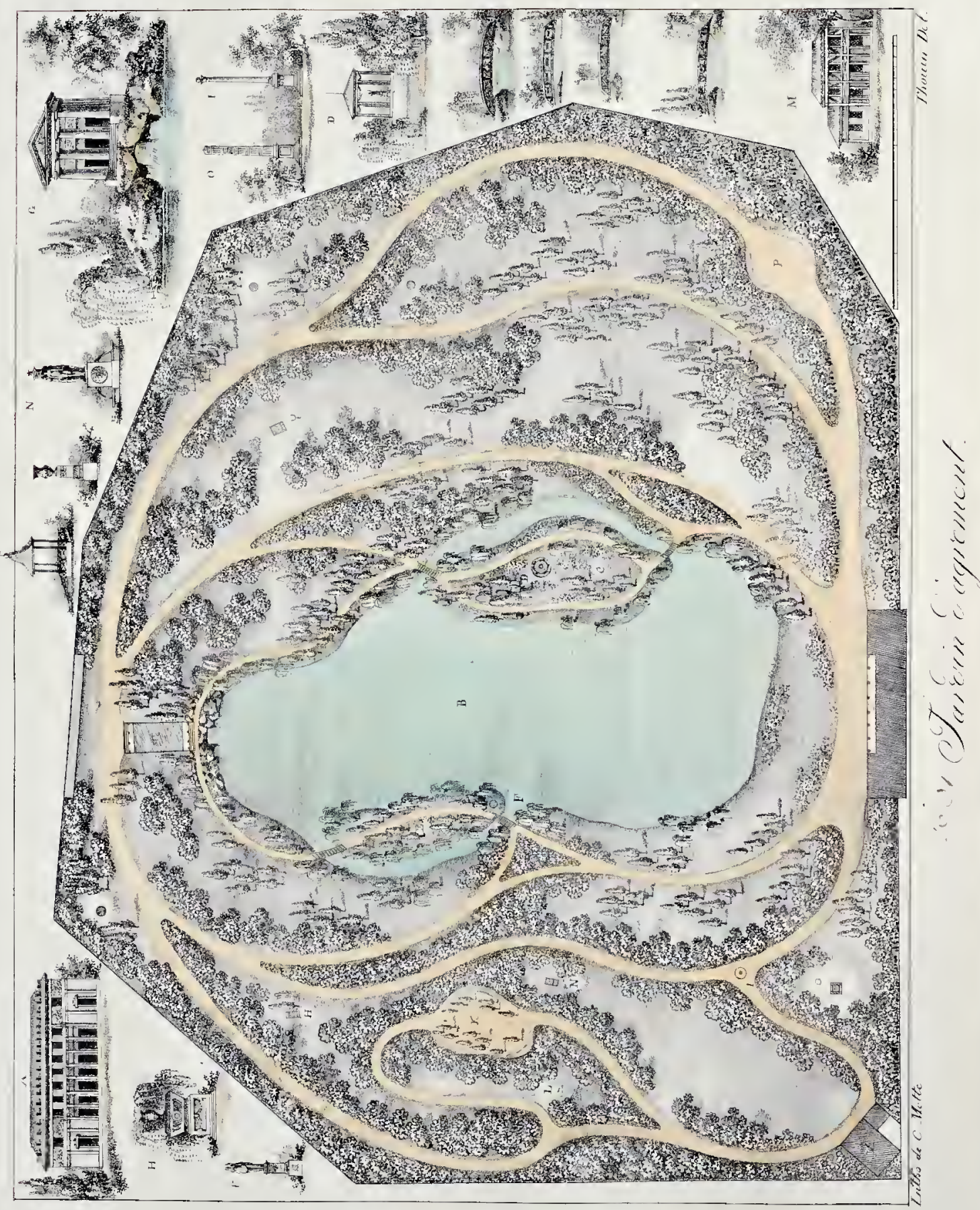




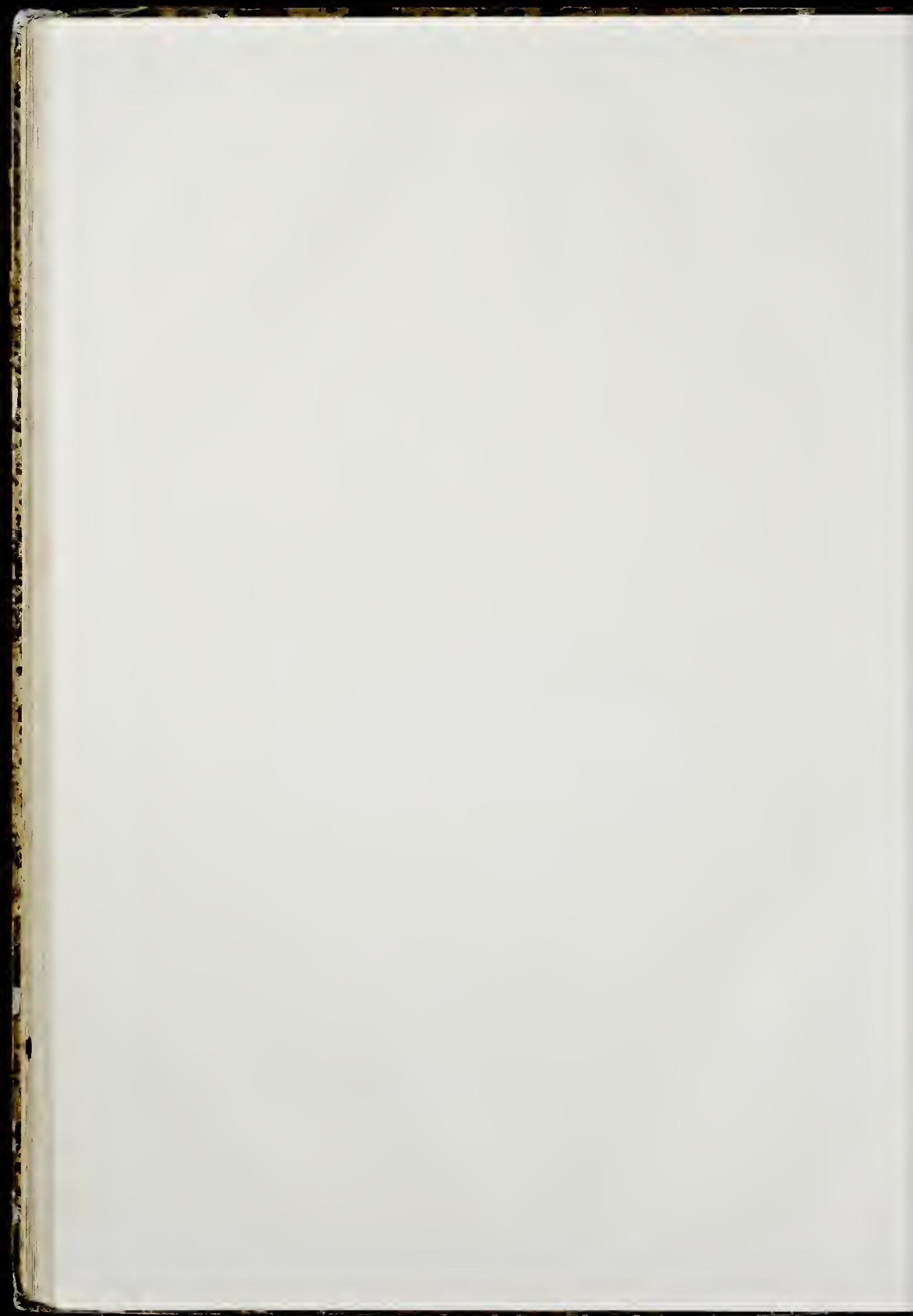




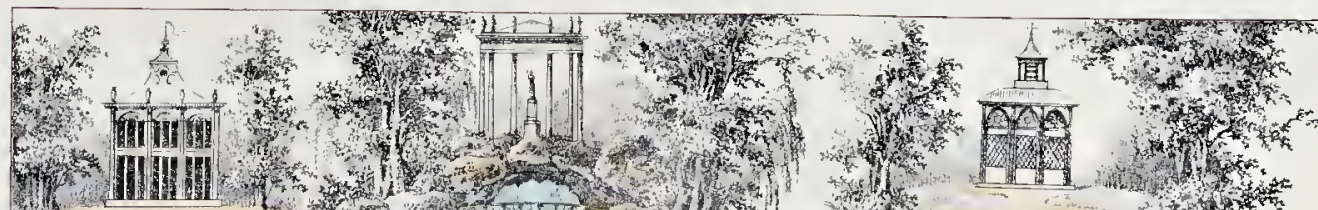

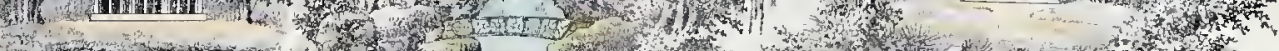

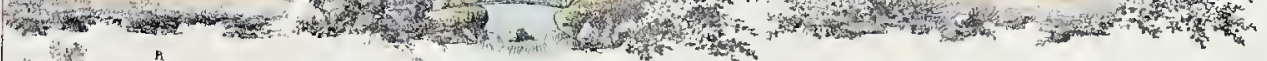

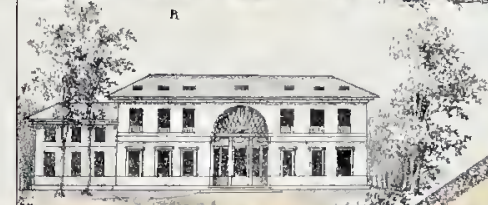

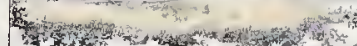

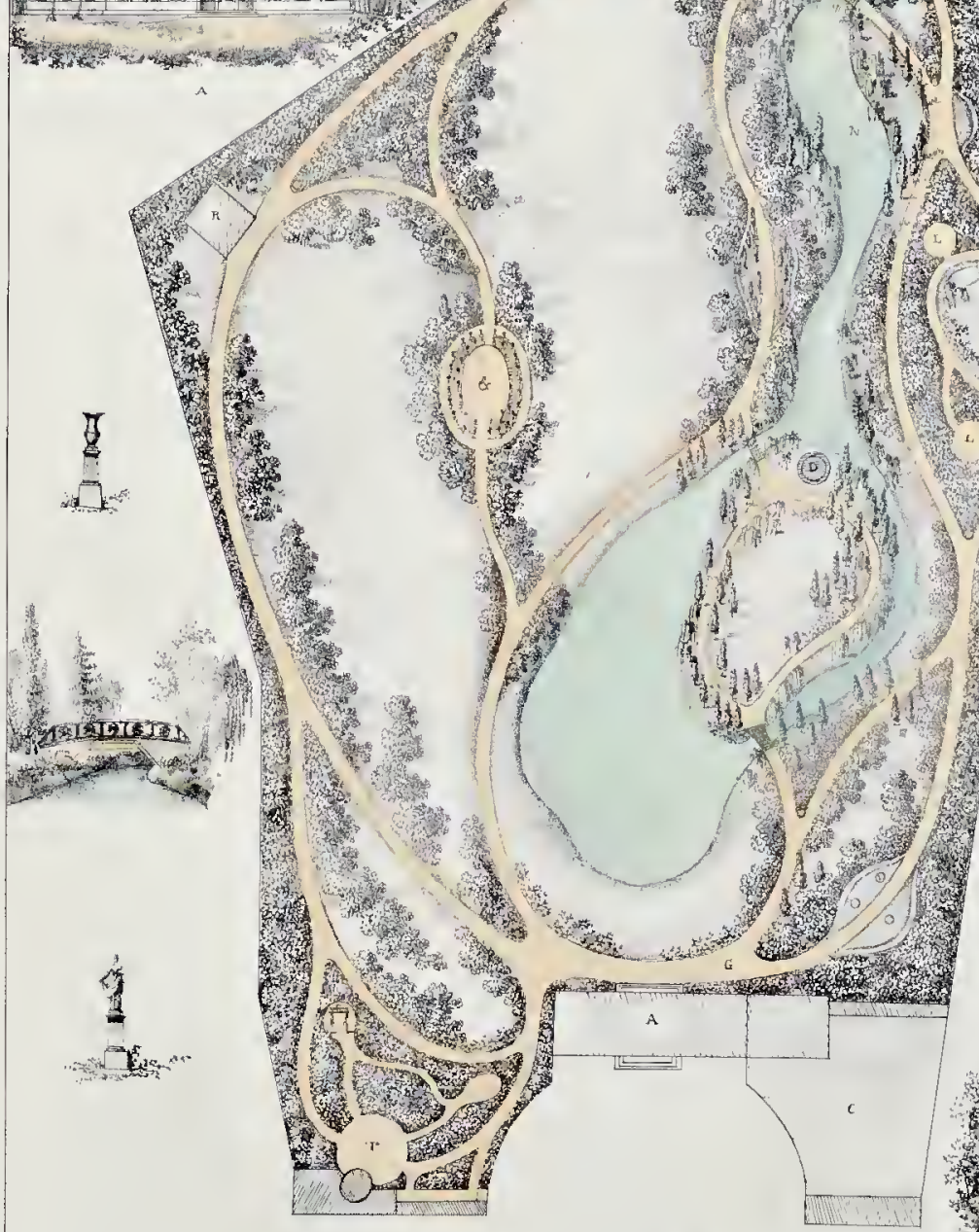

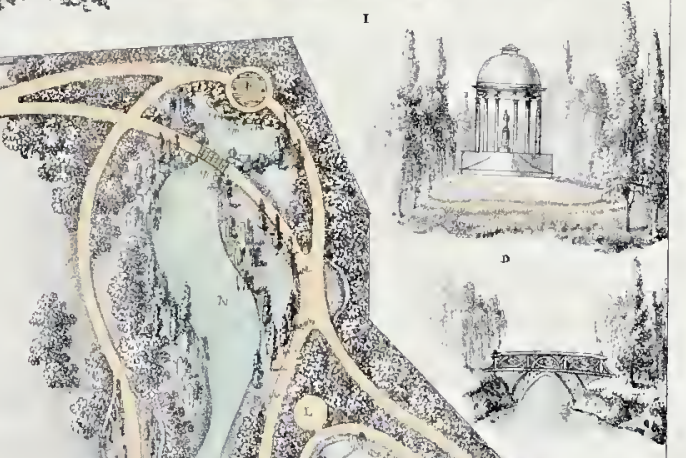
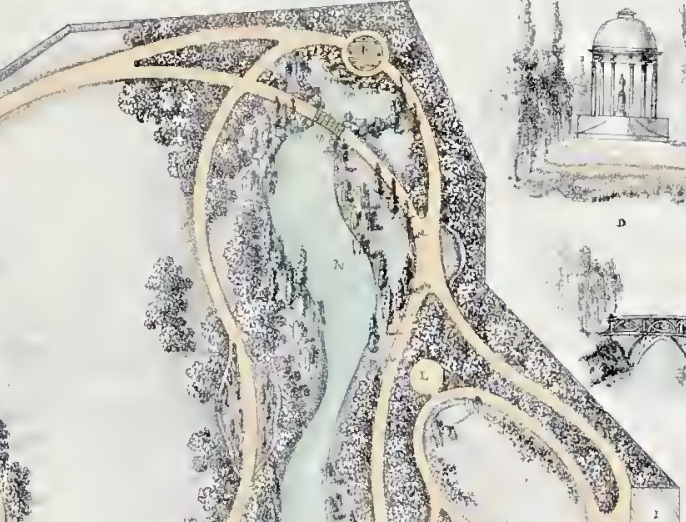

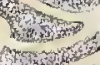

1.

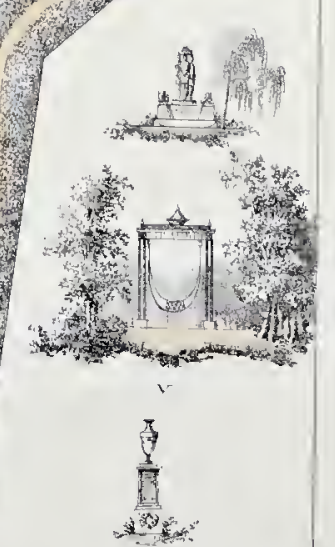

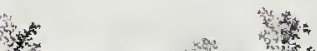

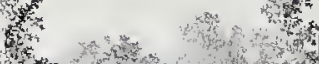

The

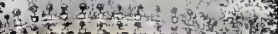

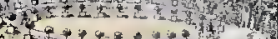

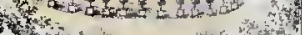
*. 


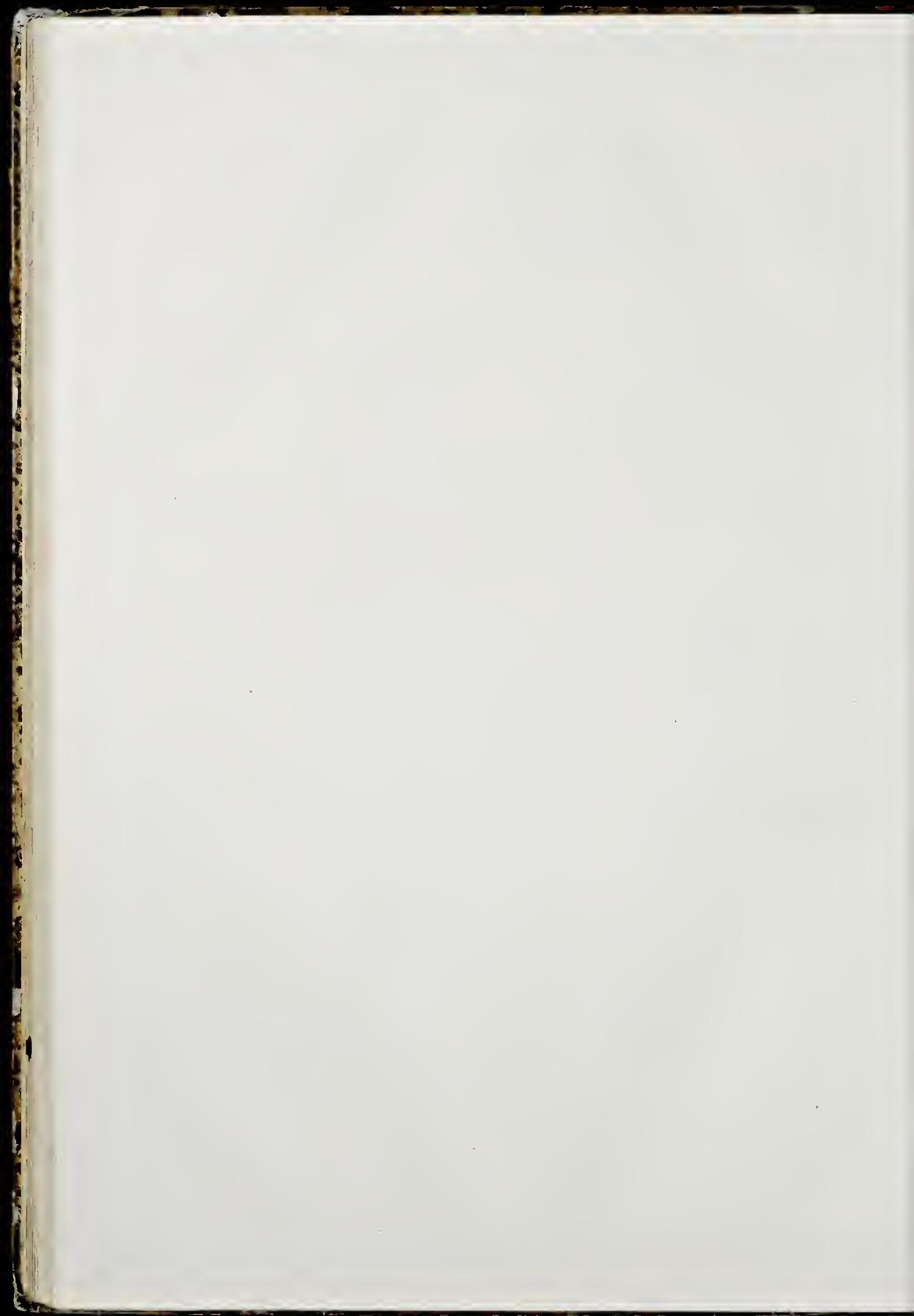




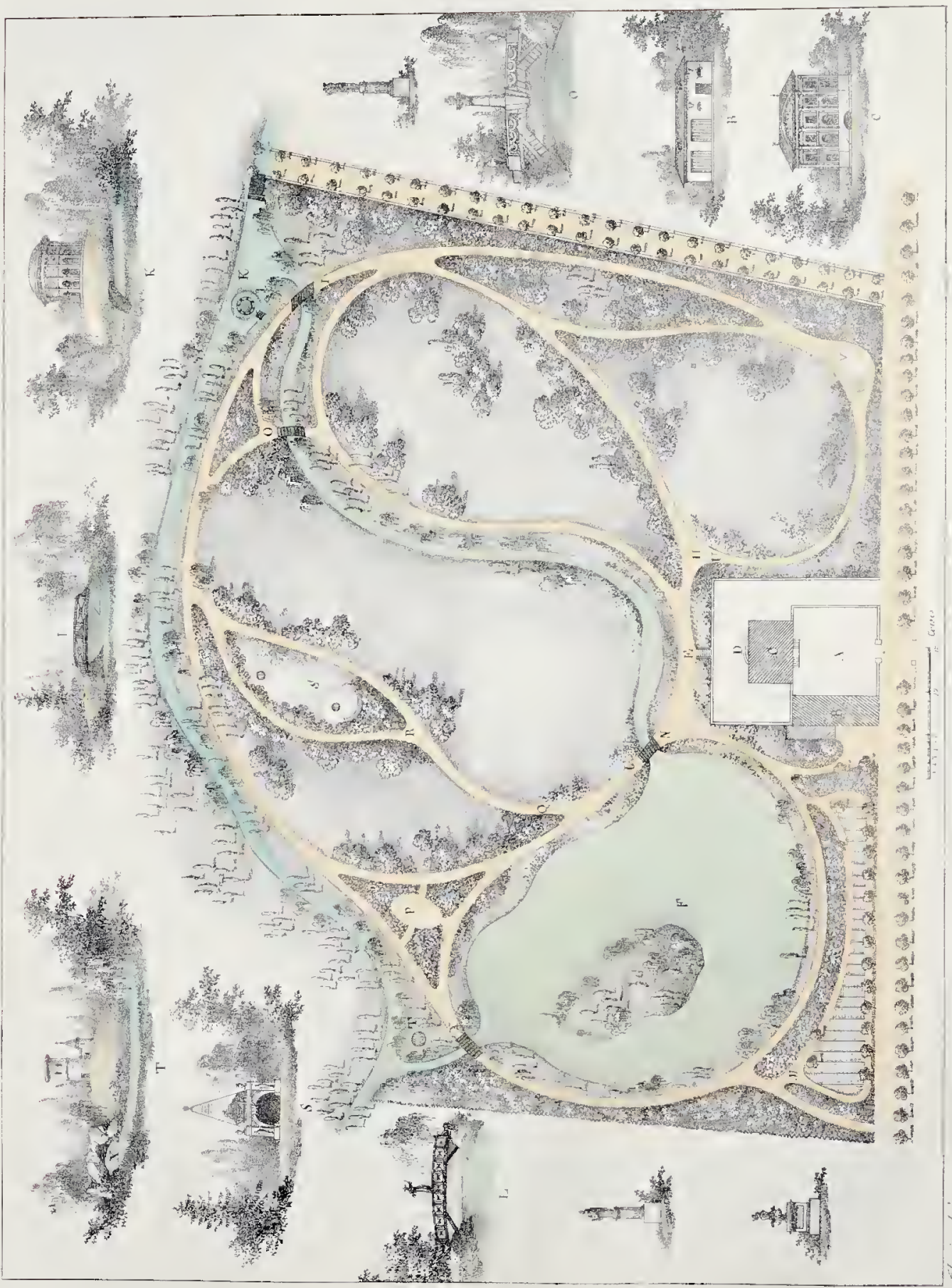




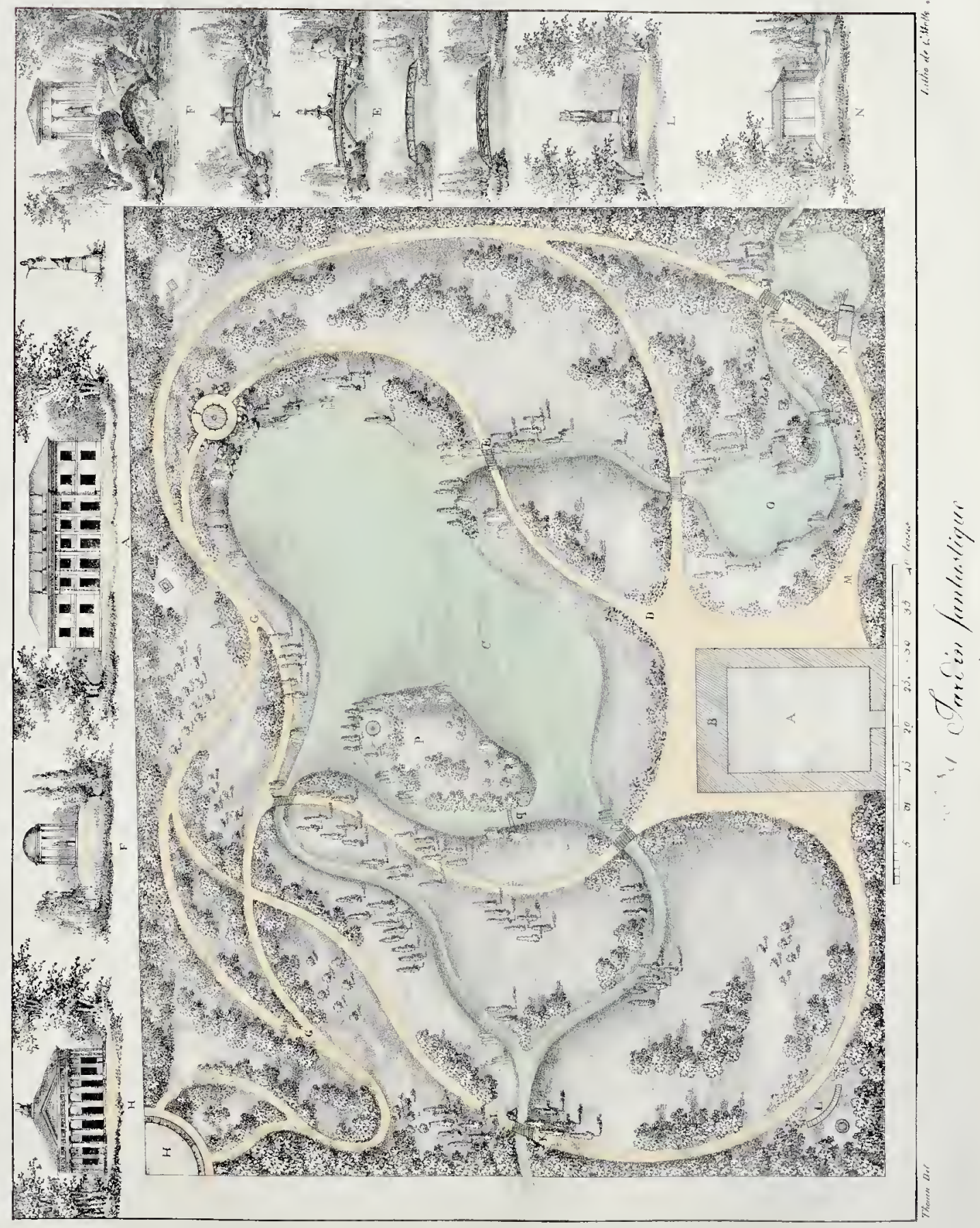




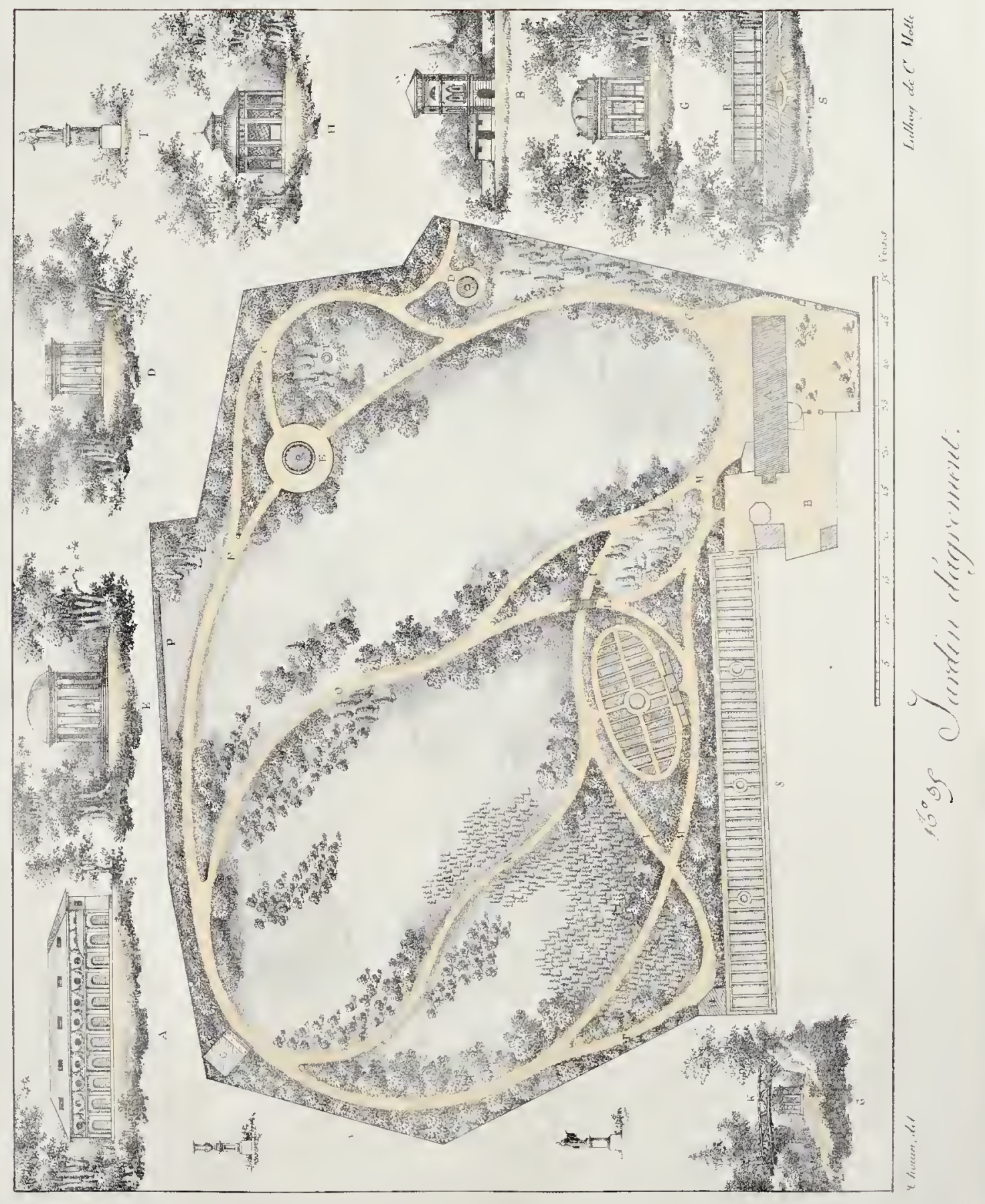




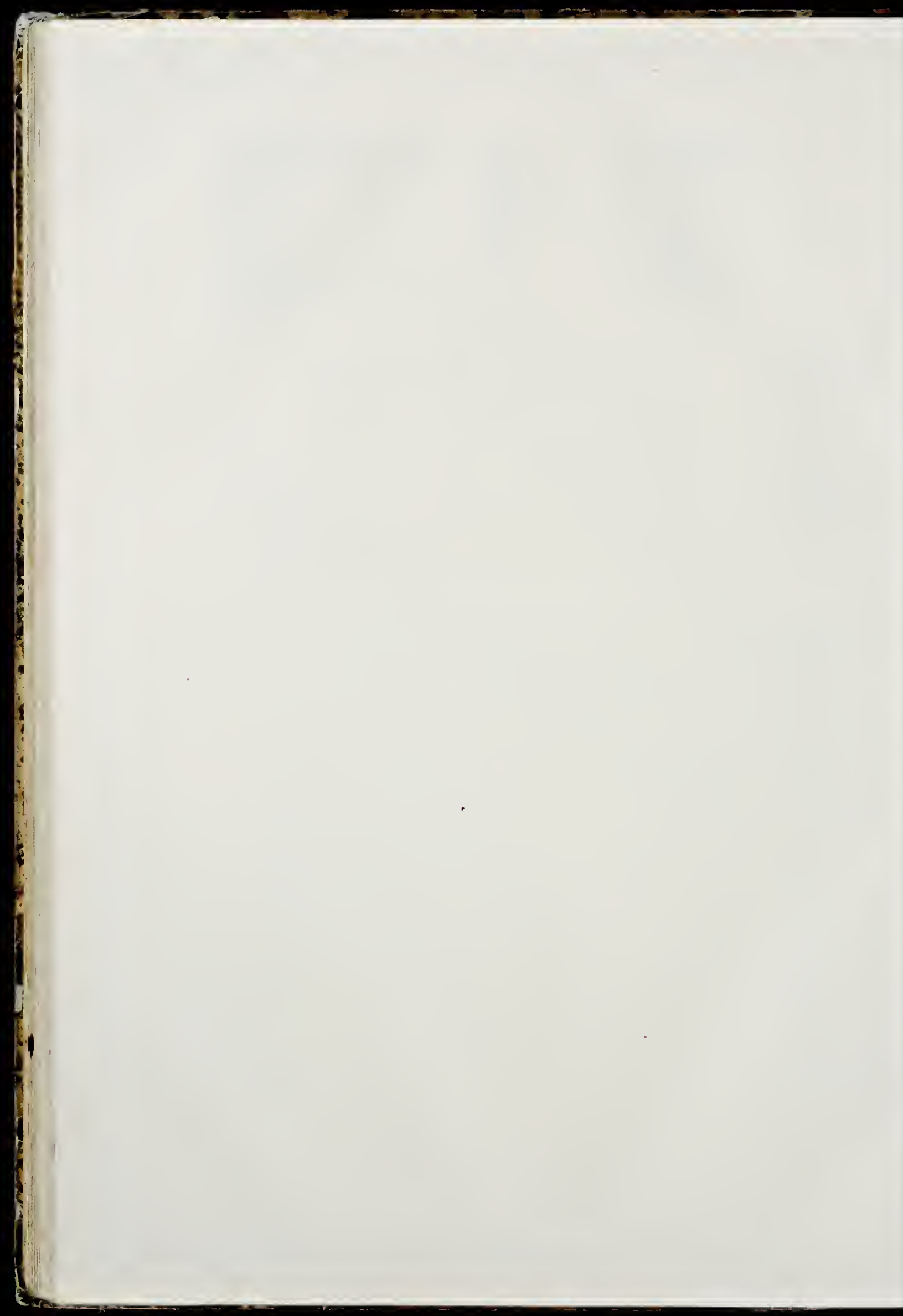




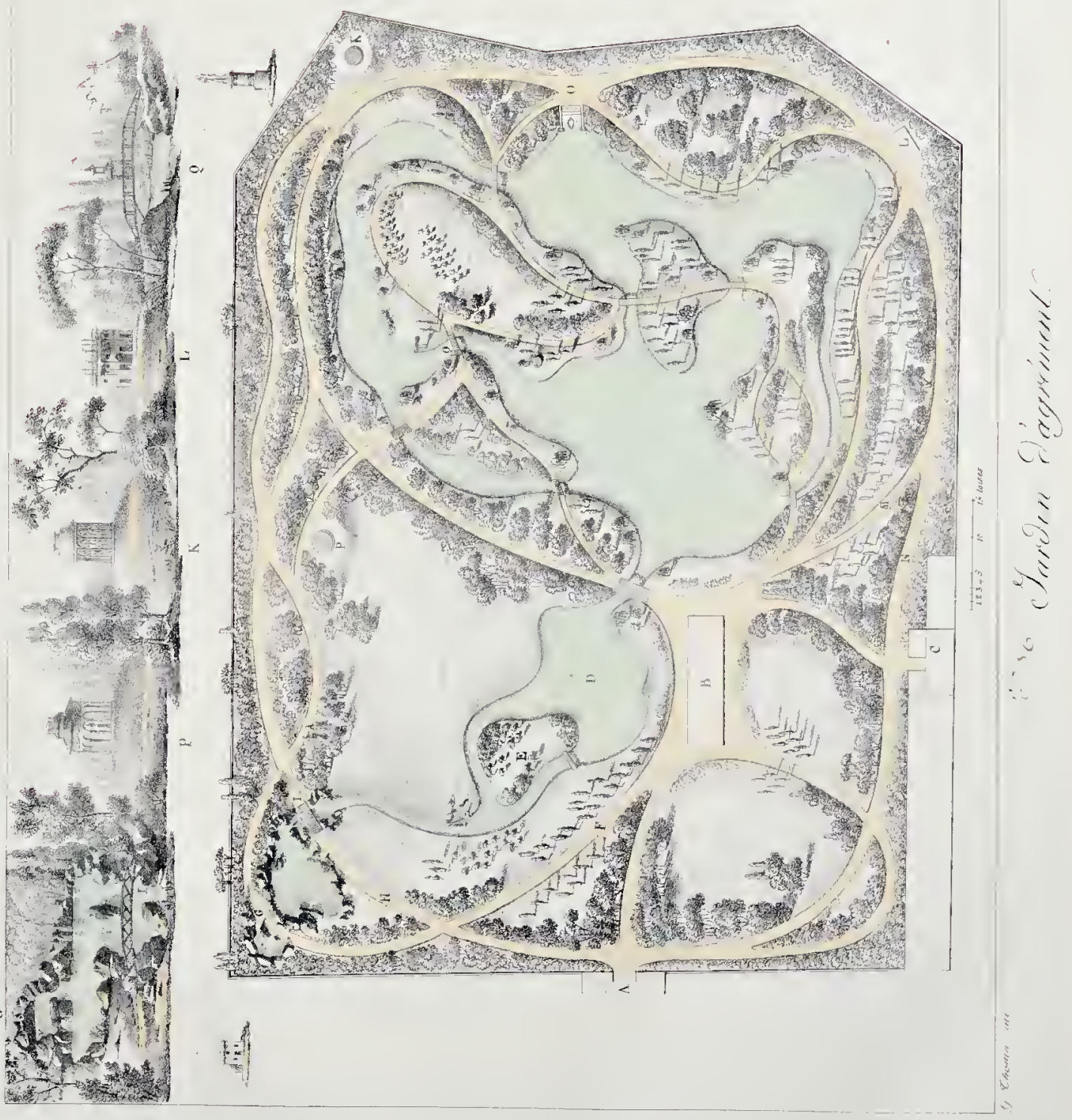




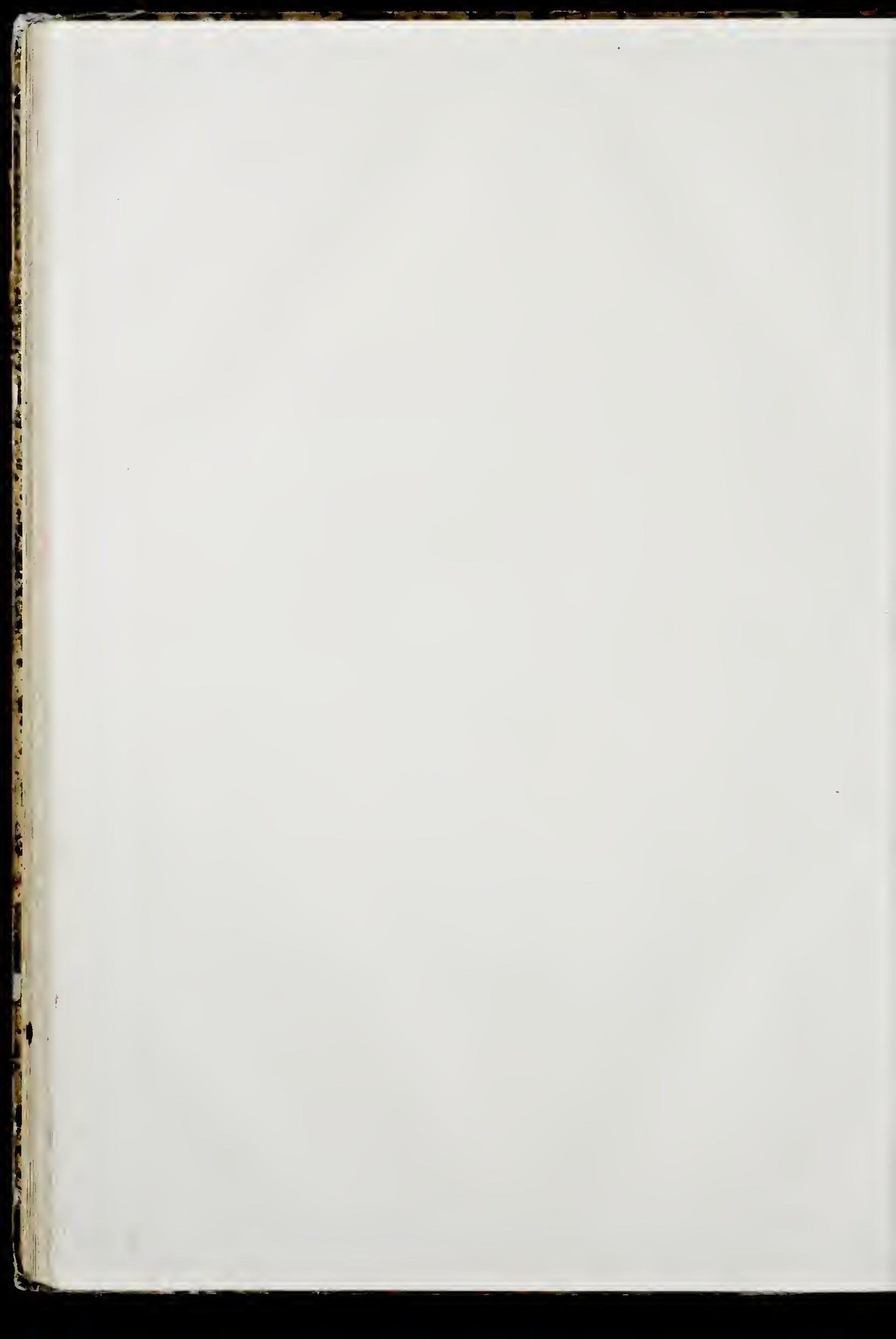




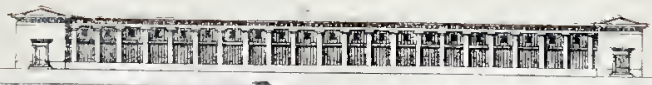

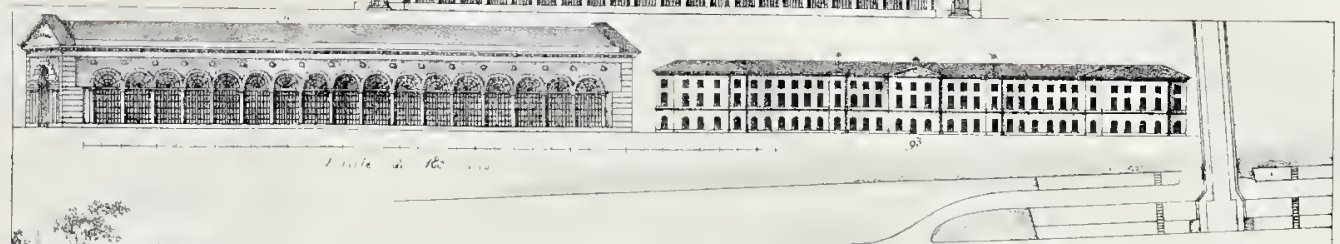

- ith

2.

A

aris

enis

4. 1 .

12 T.

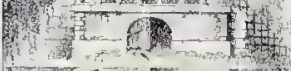

4)

W.

tat on

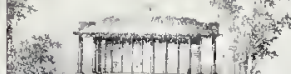

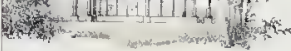

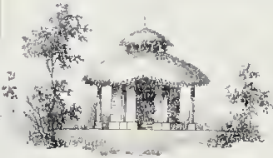

1.

L.

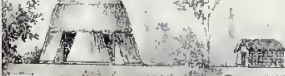

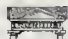

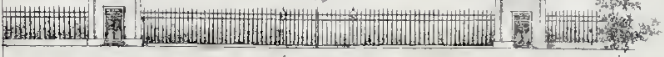

息

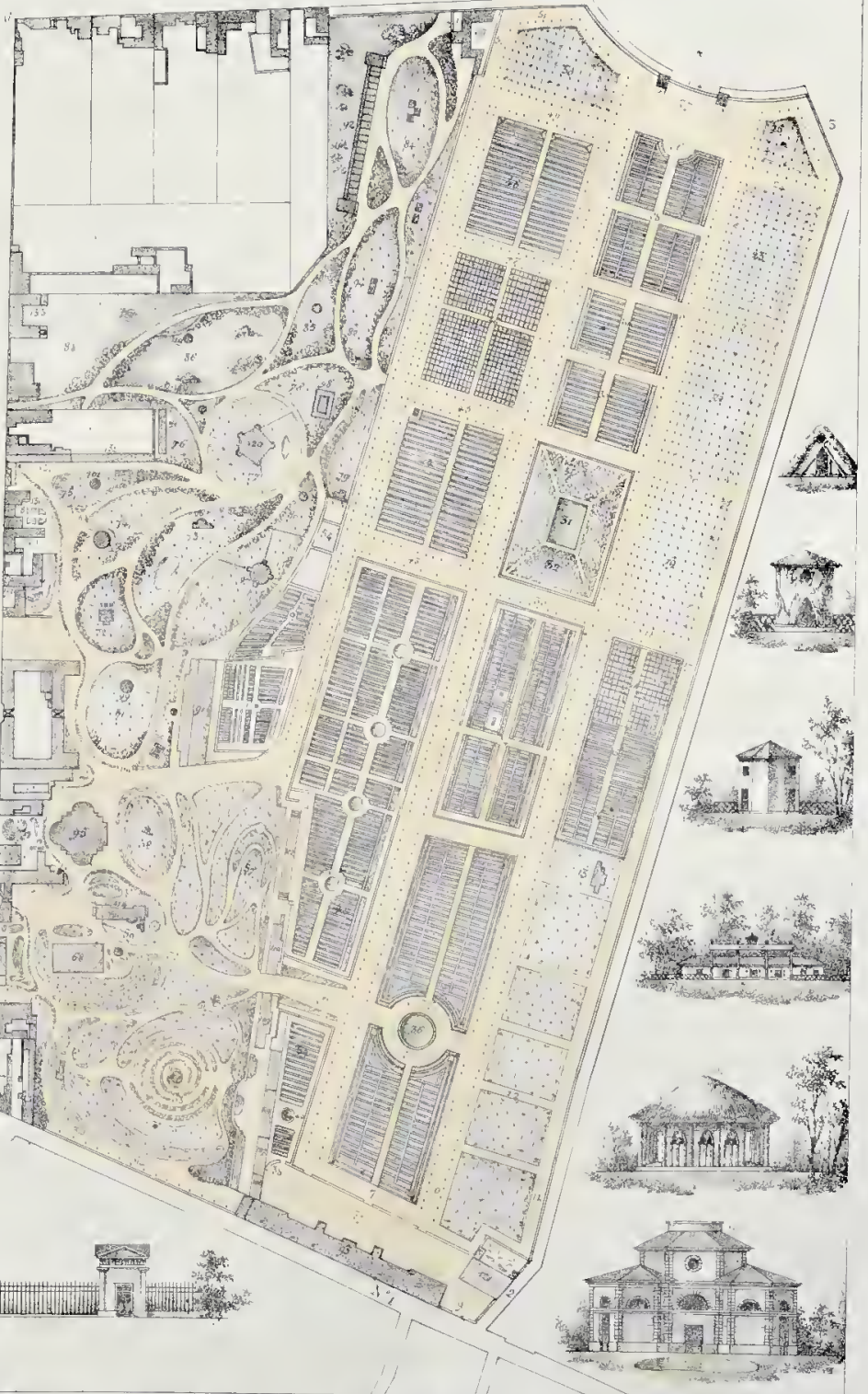

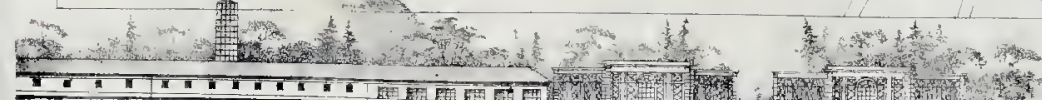

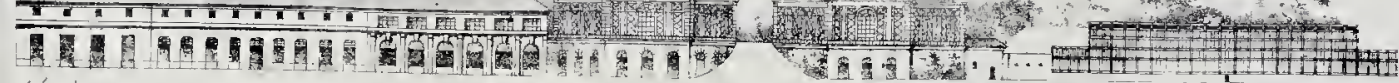

$$
\text { trialinile Cris. }
$$




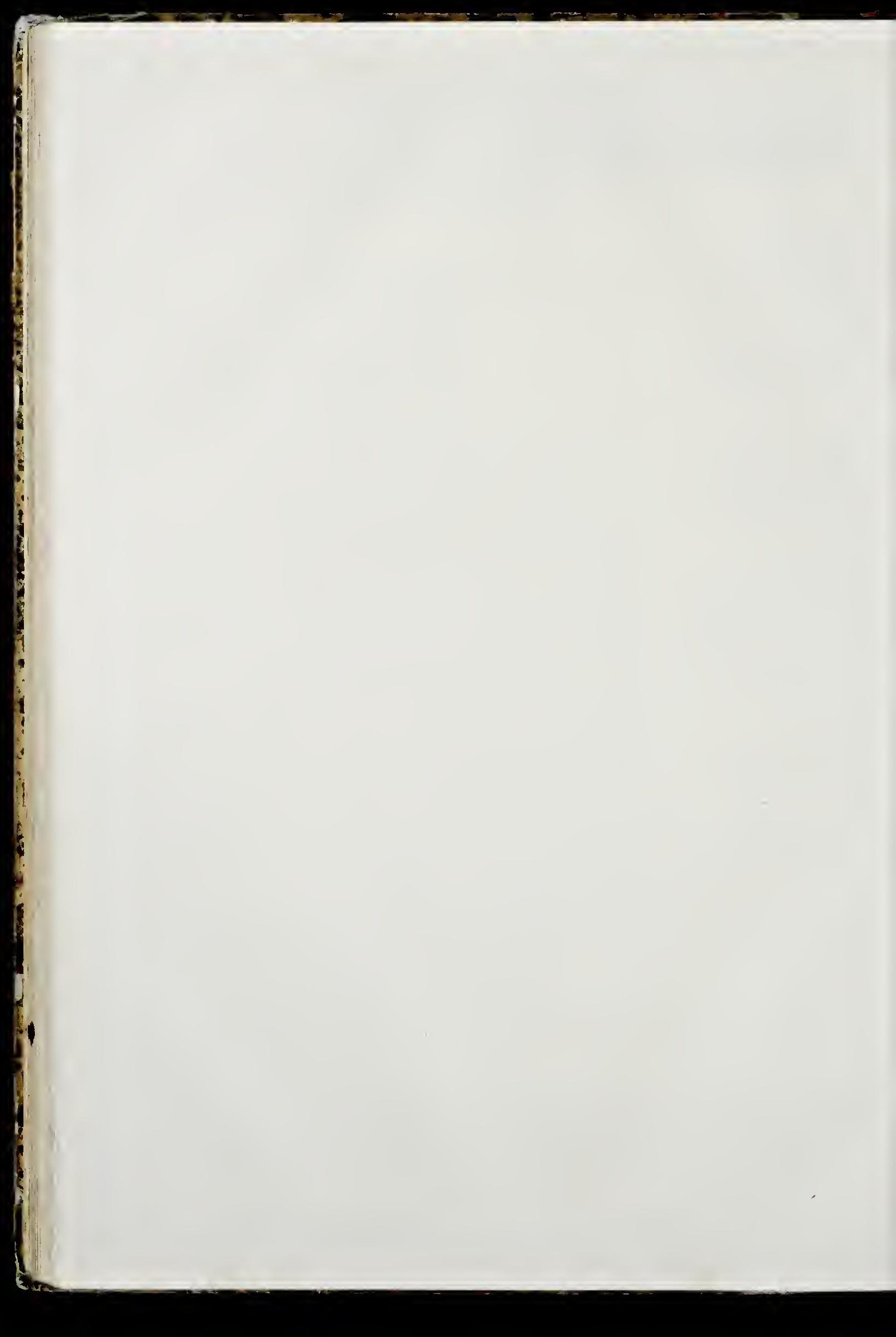




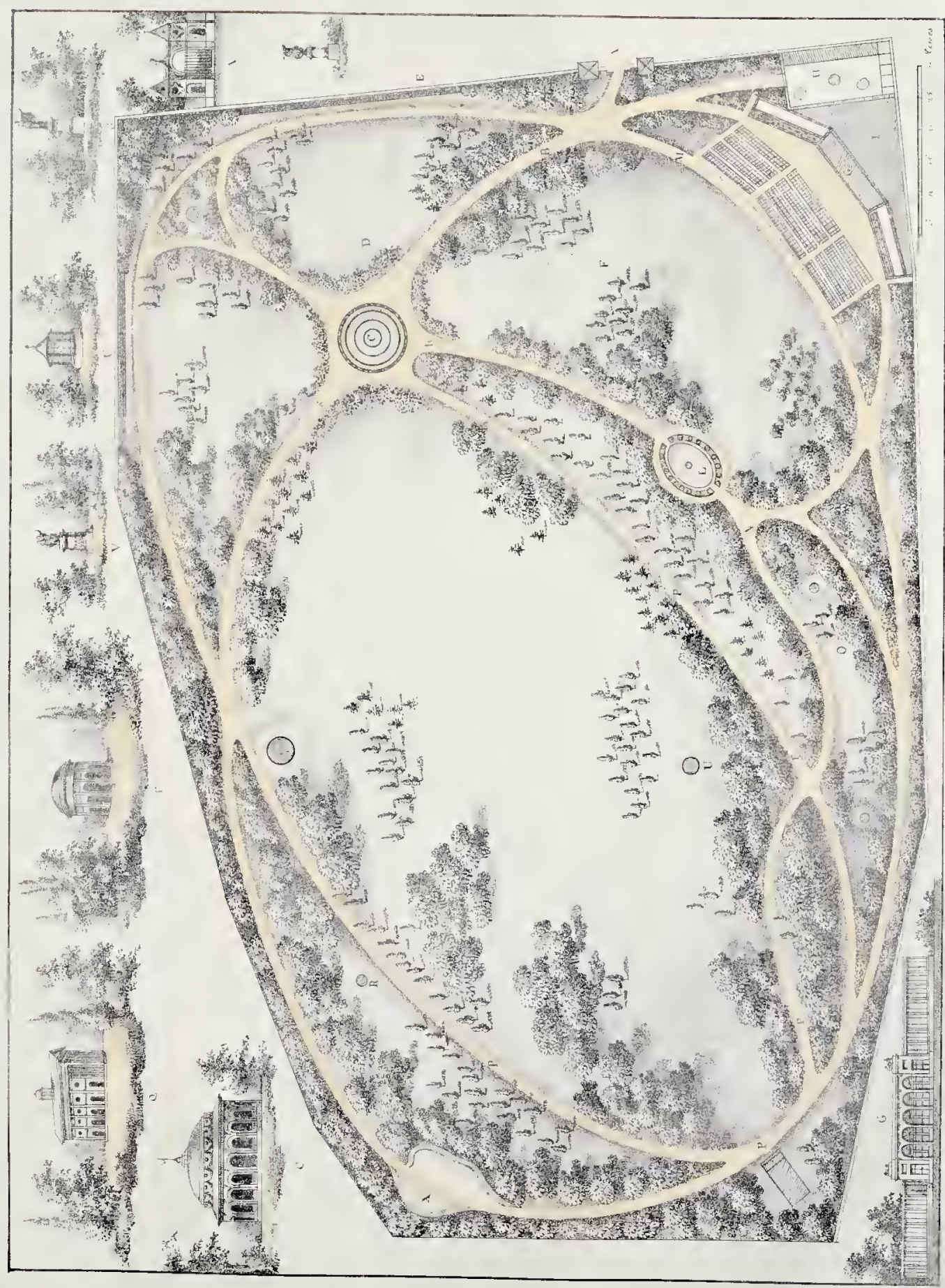




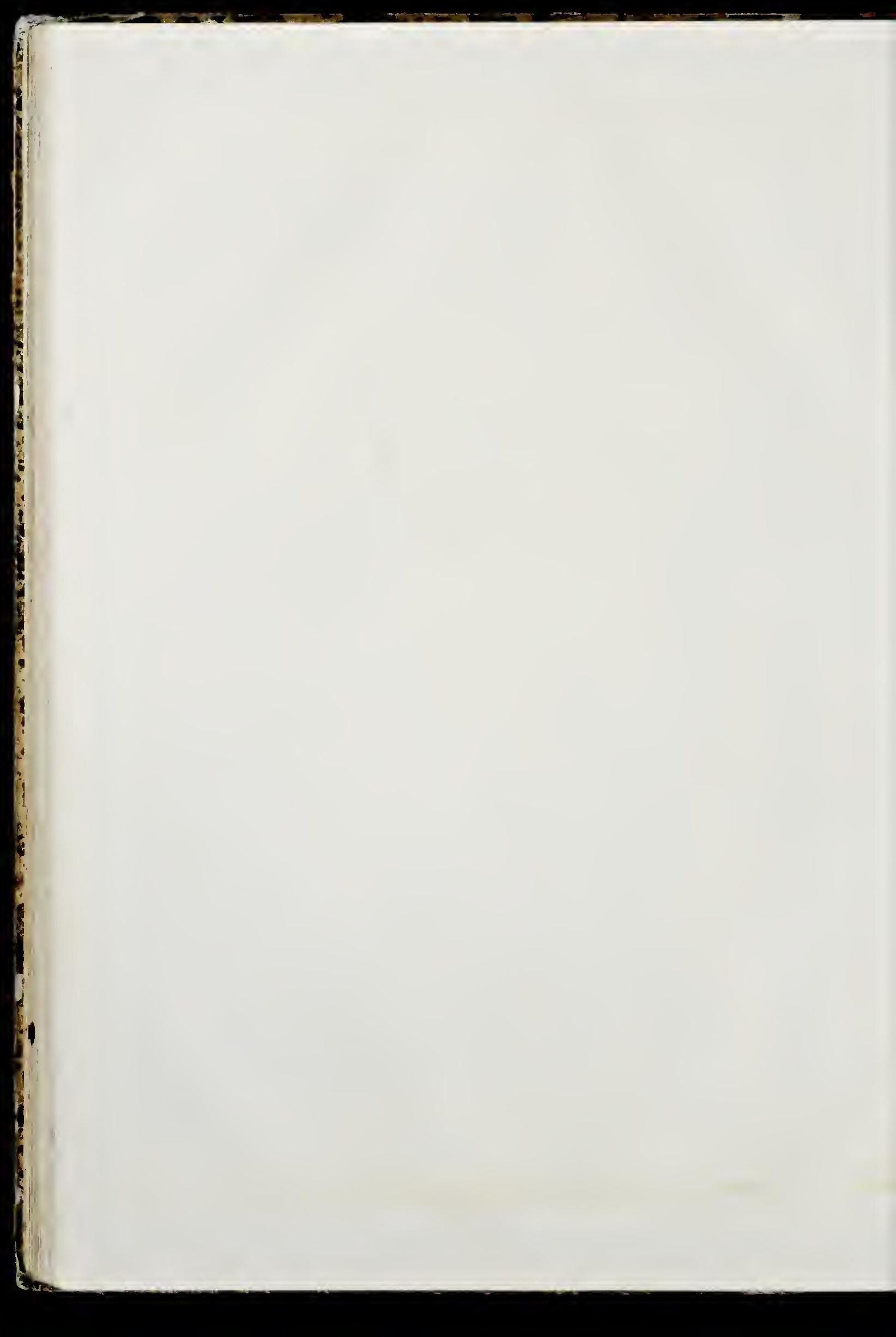




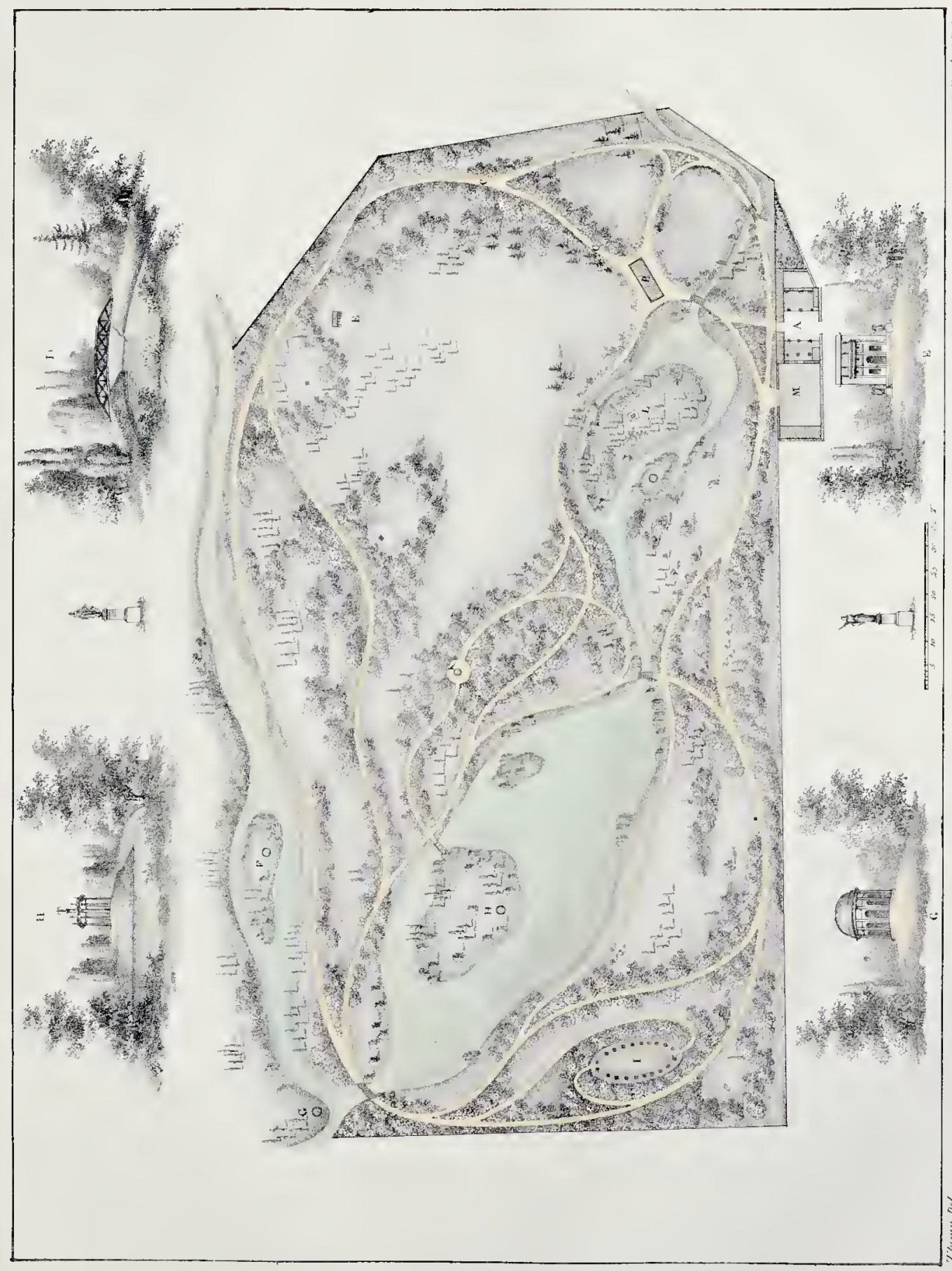




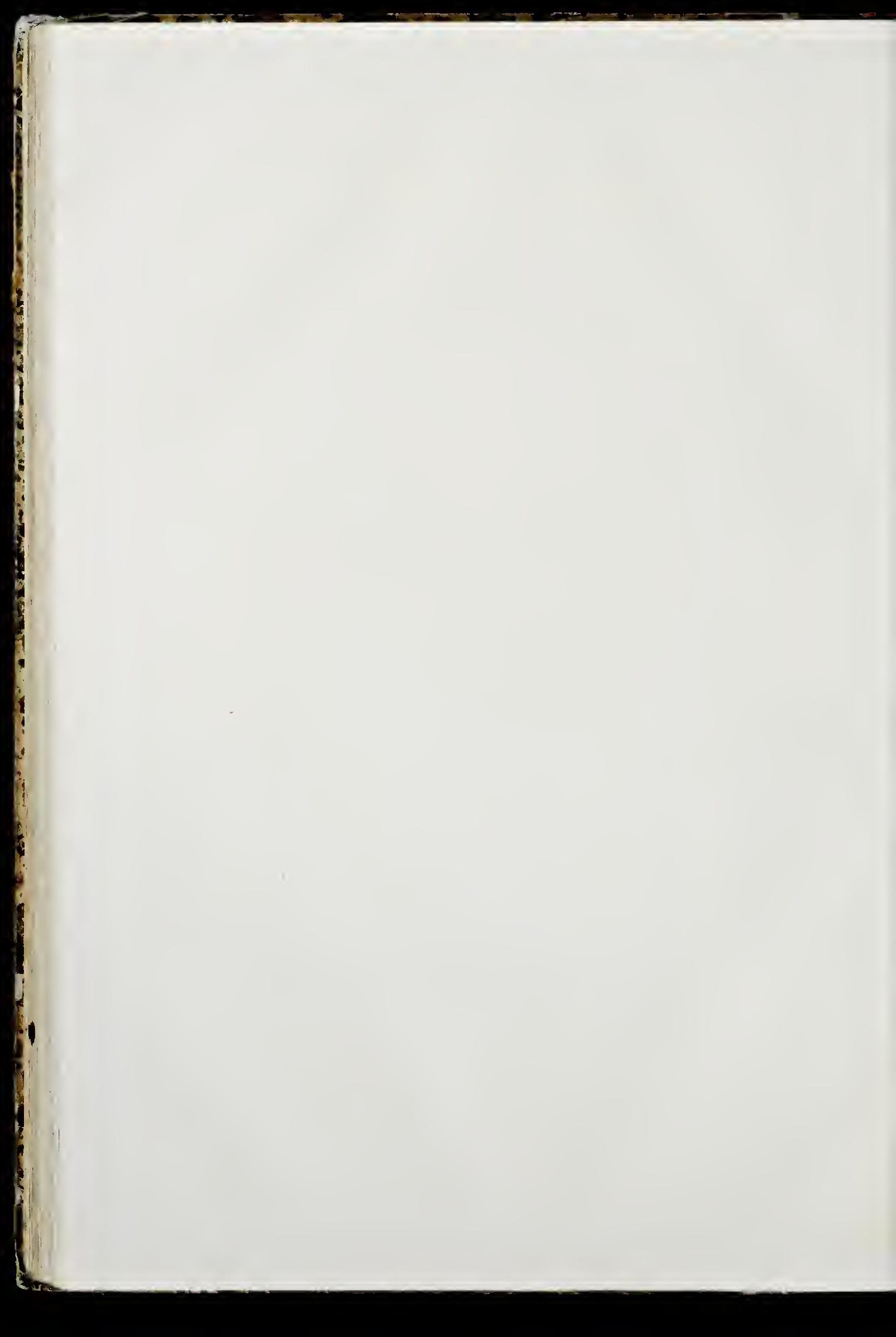




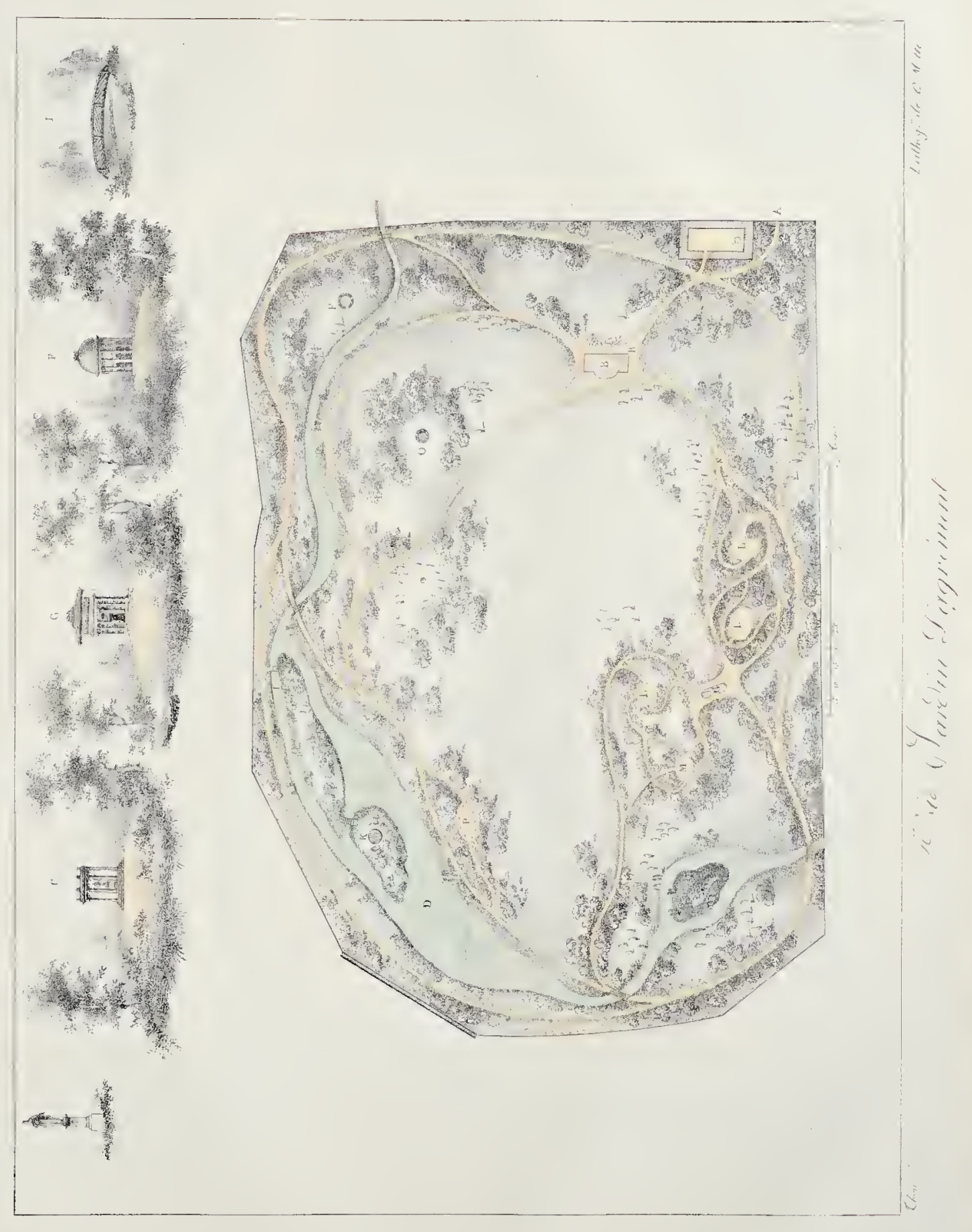




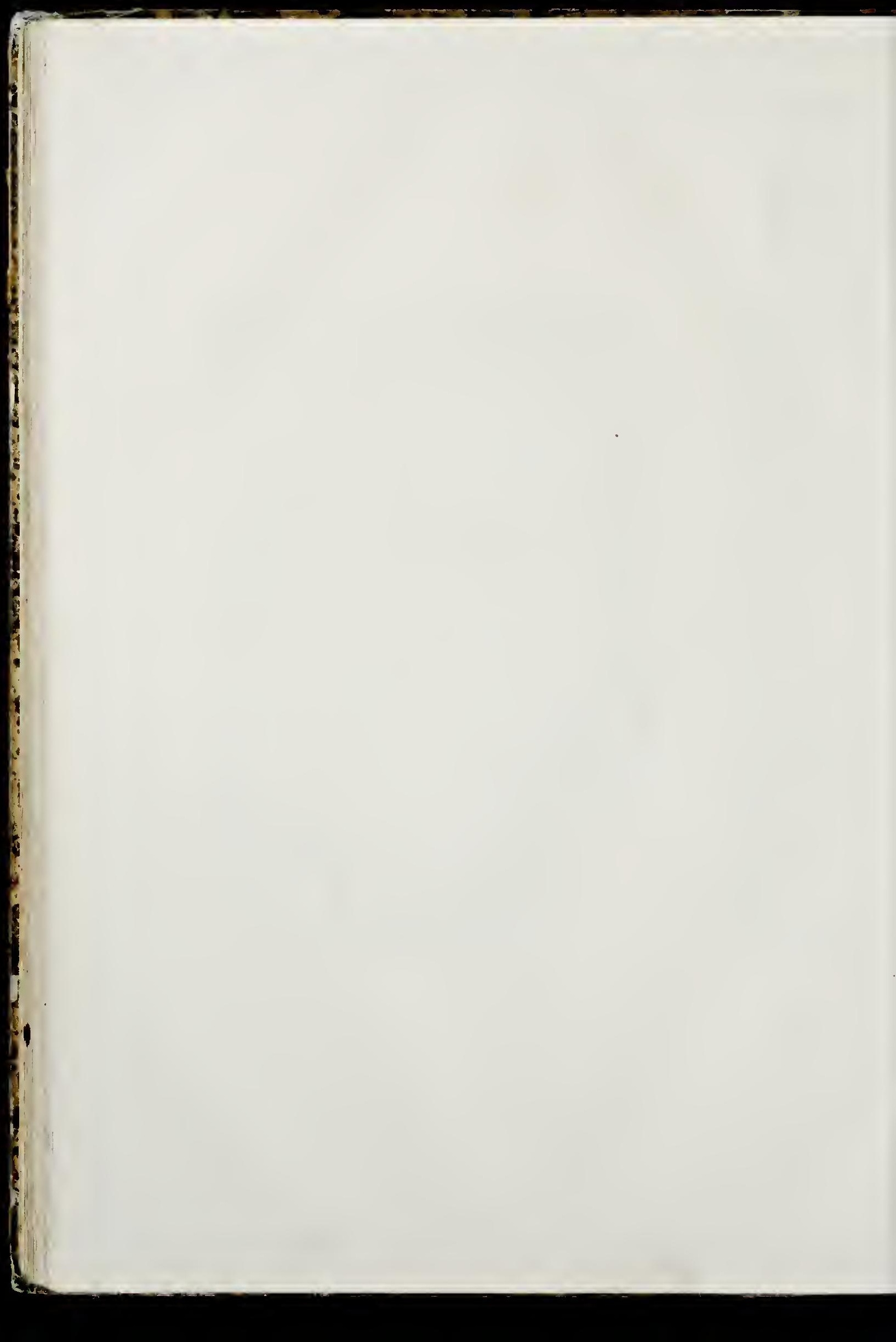




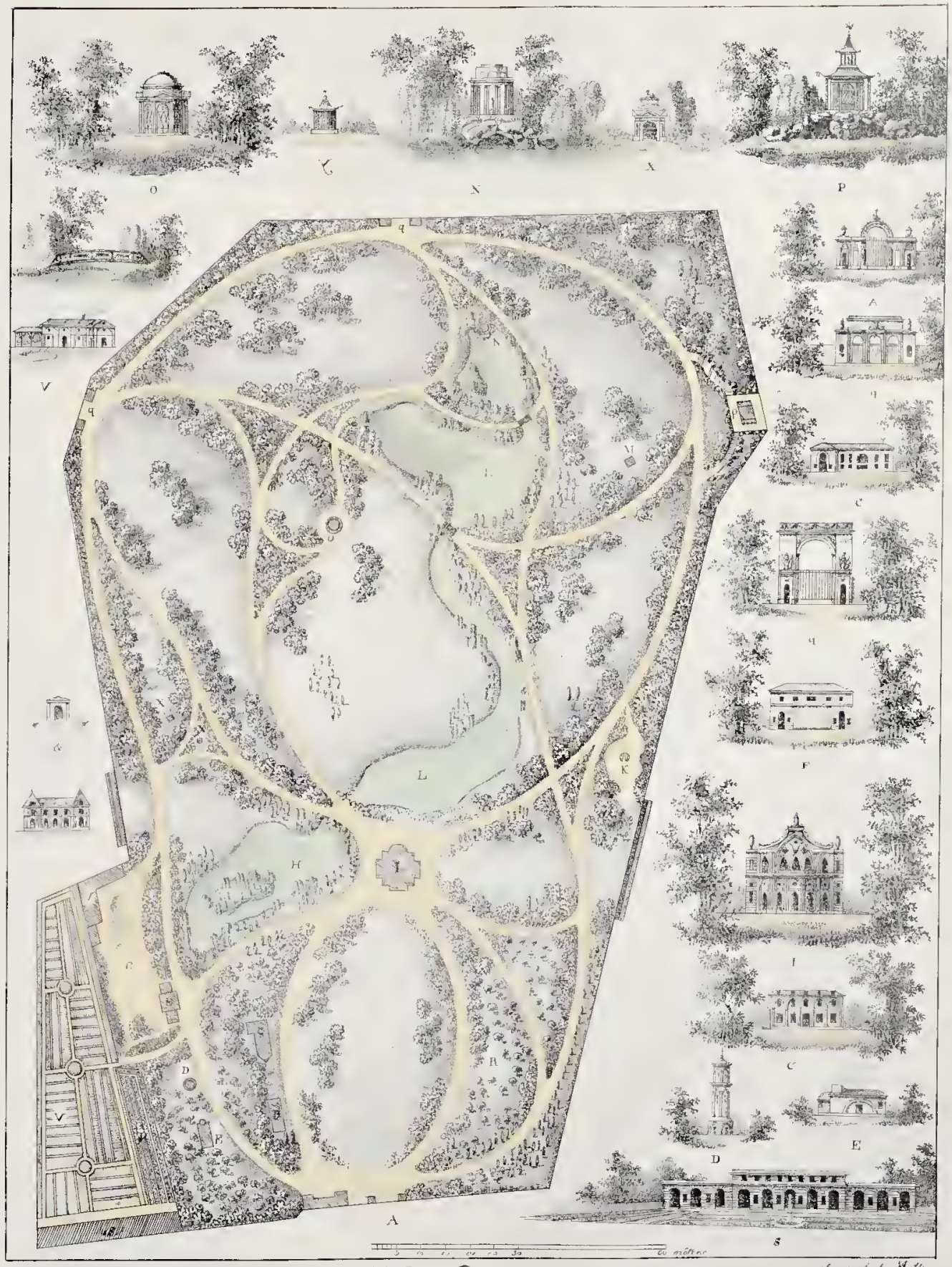




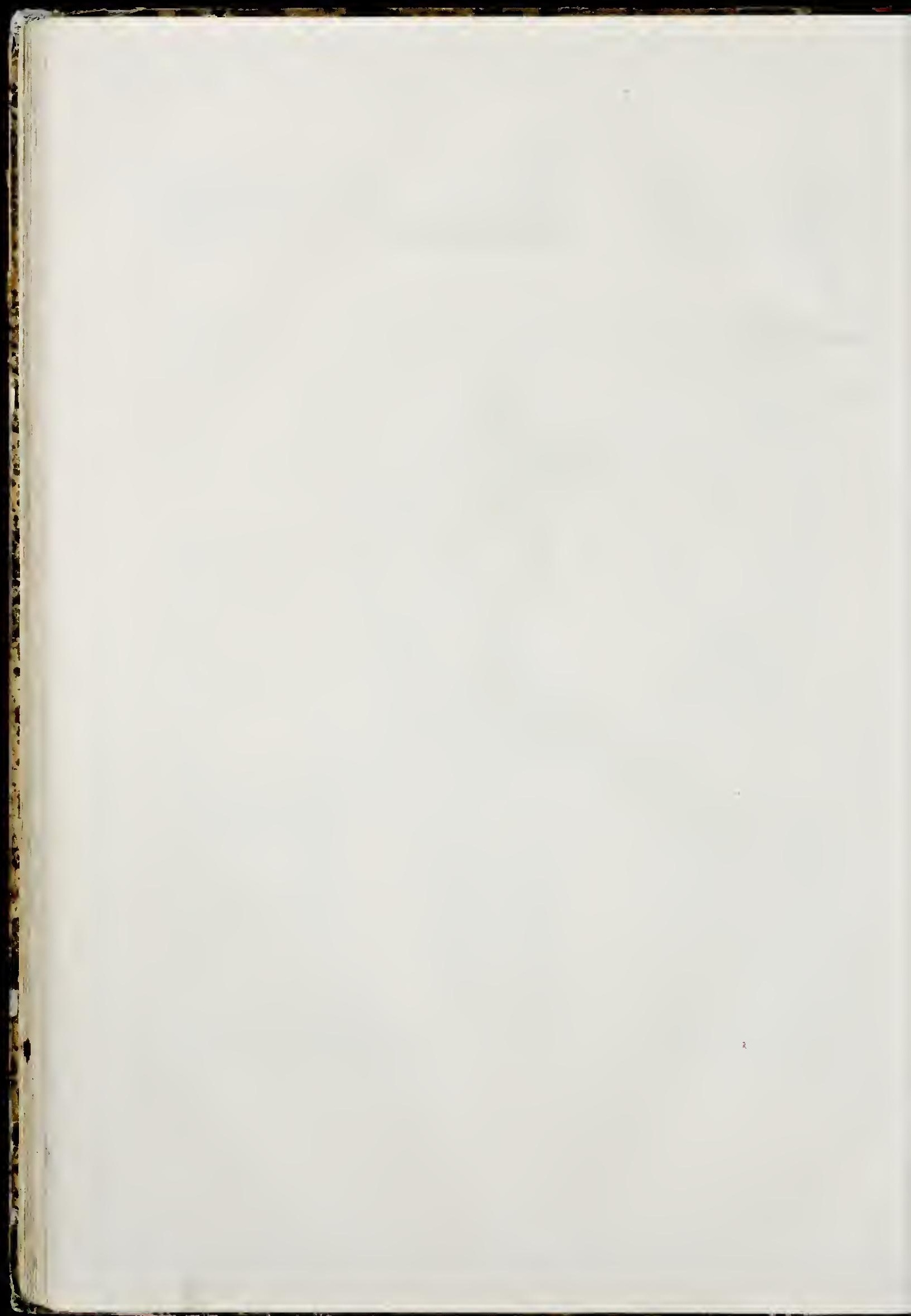




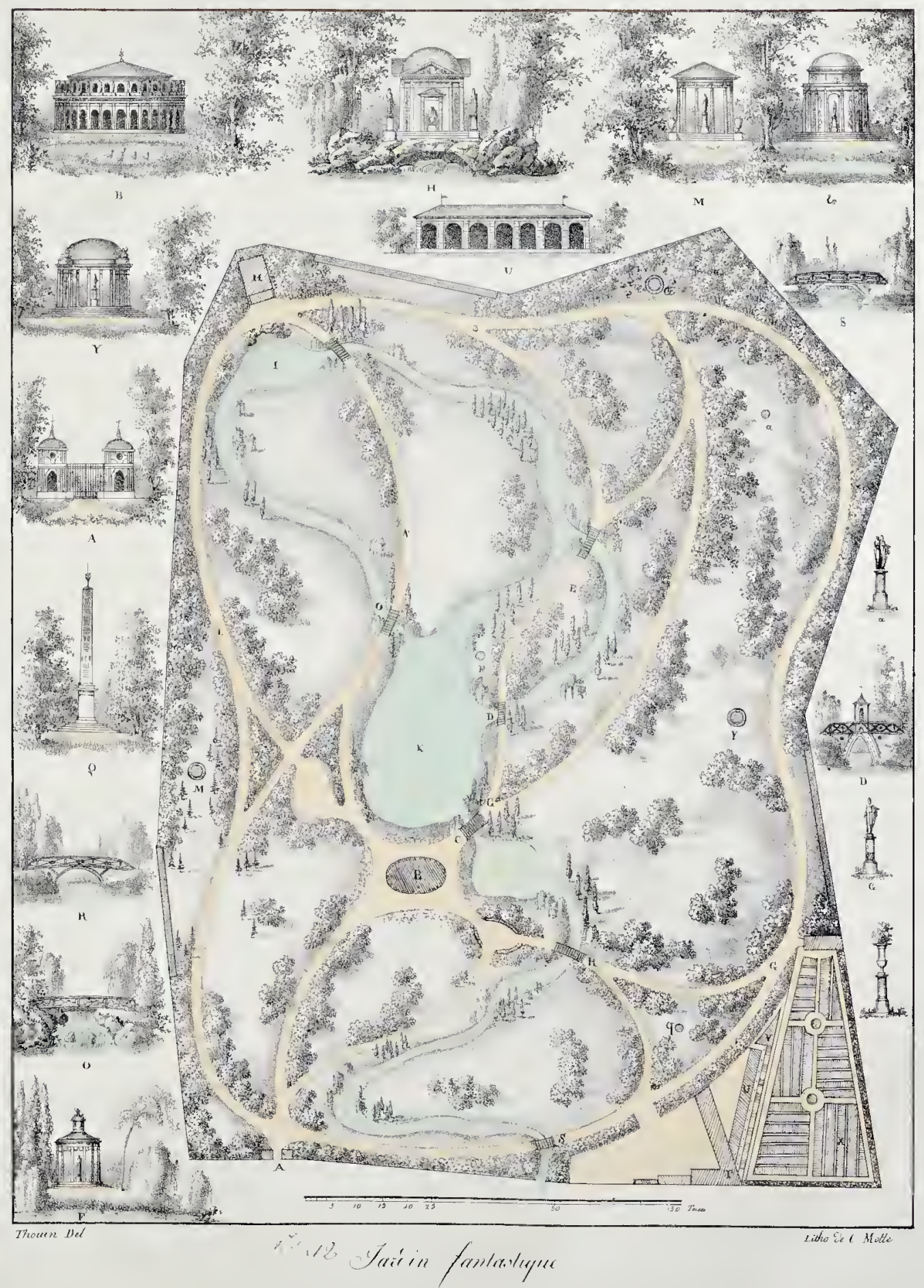




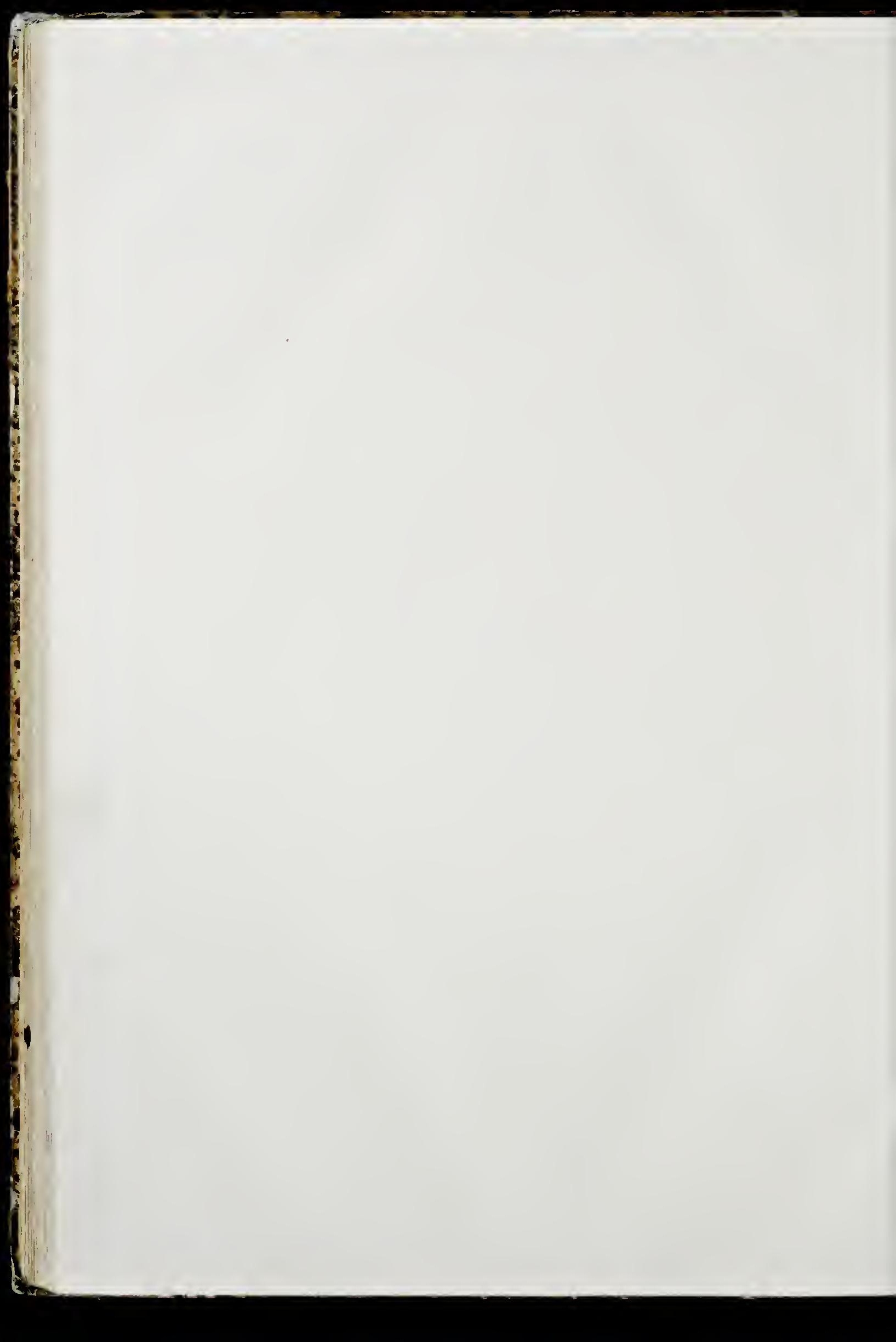




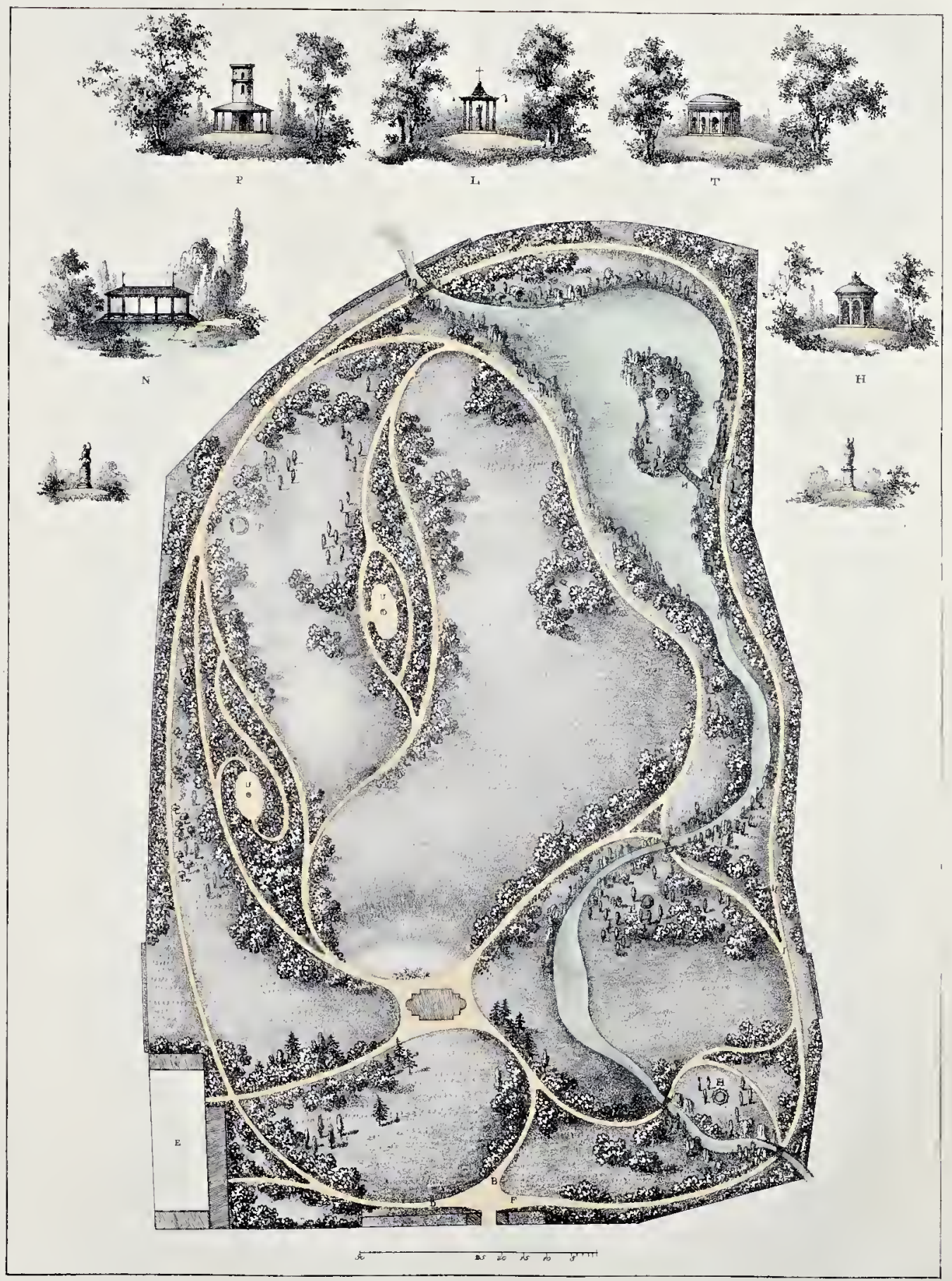

is Ofrodion digresnent 


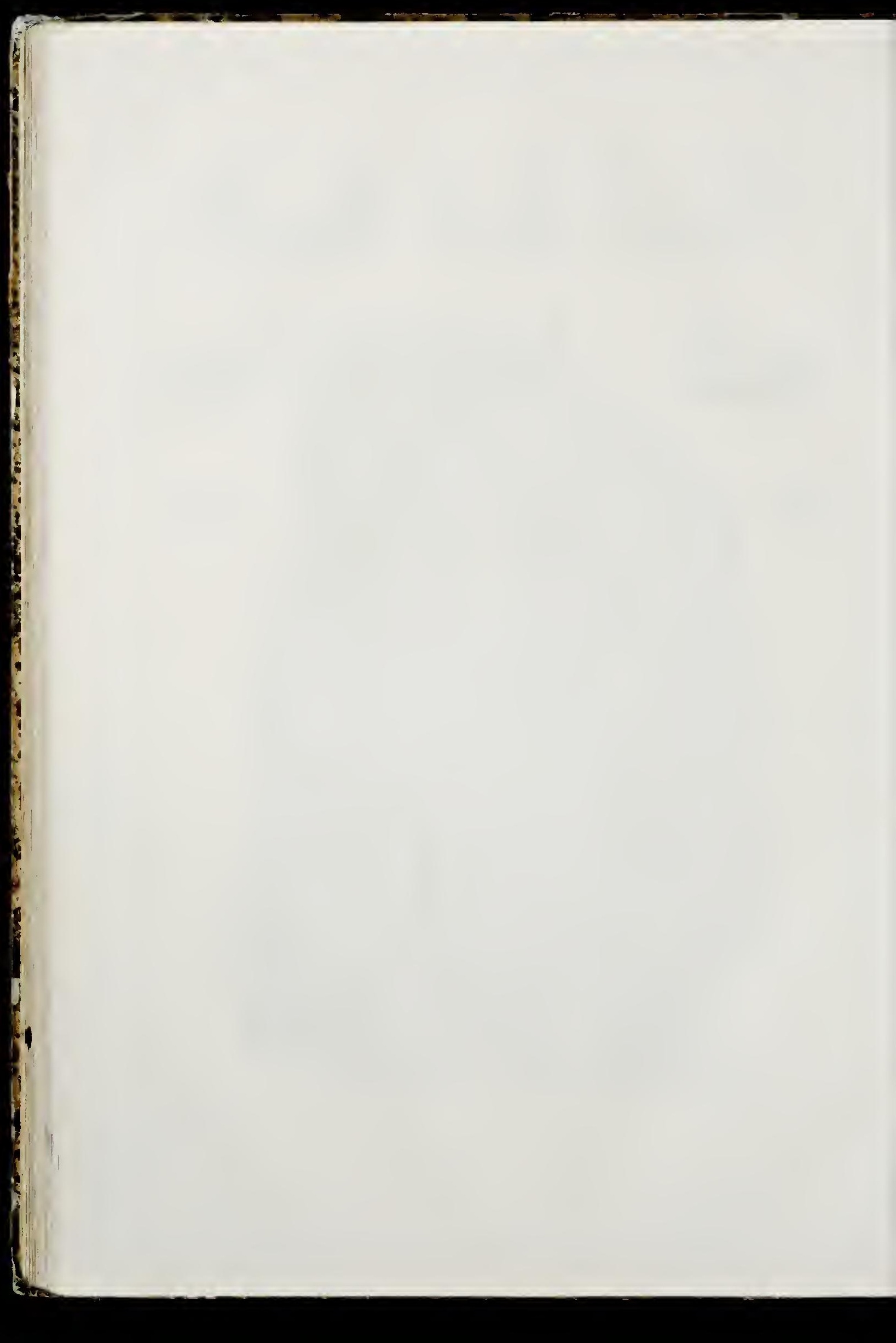




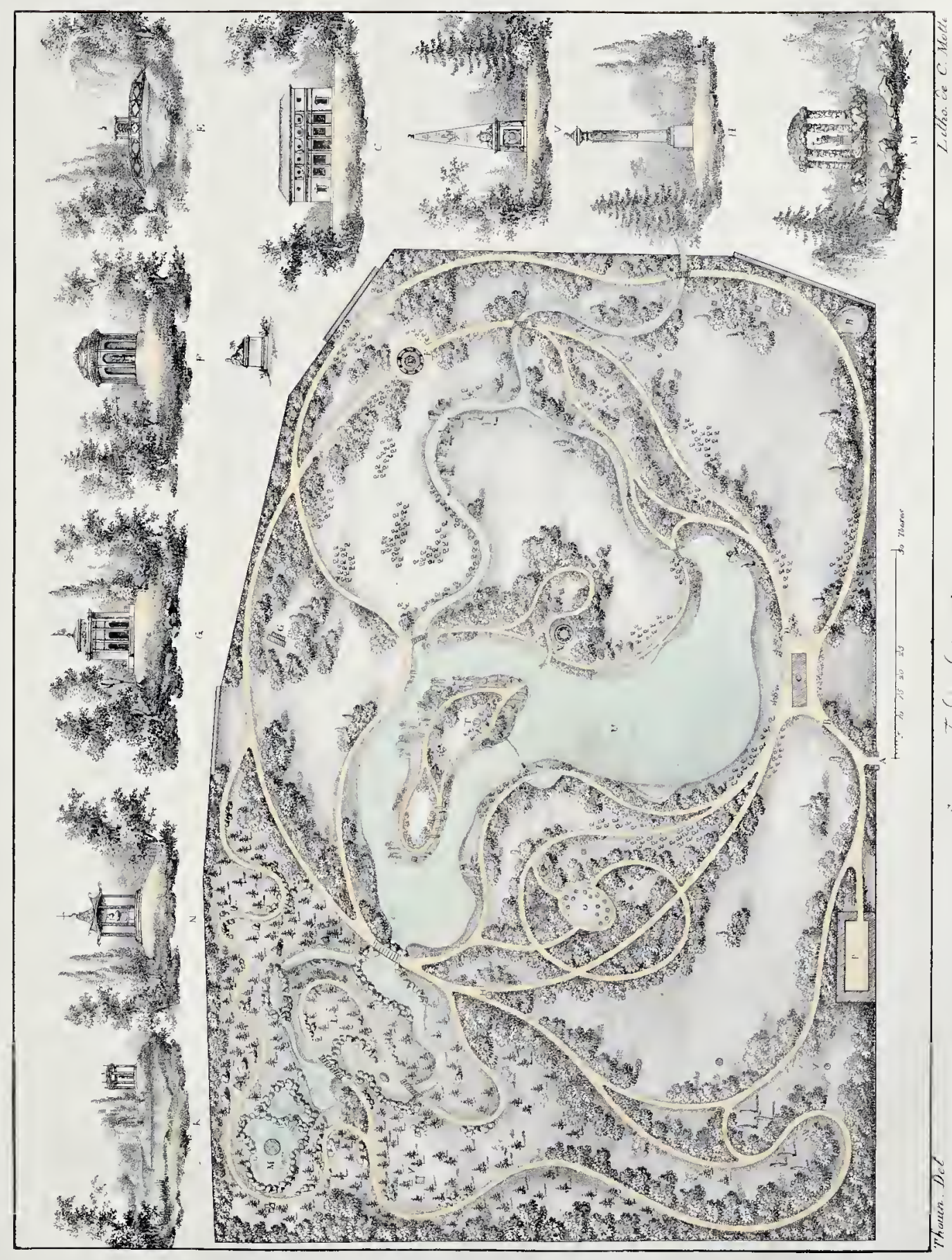




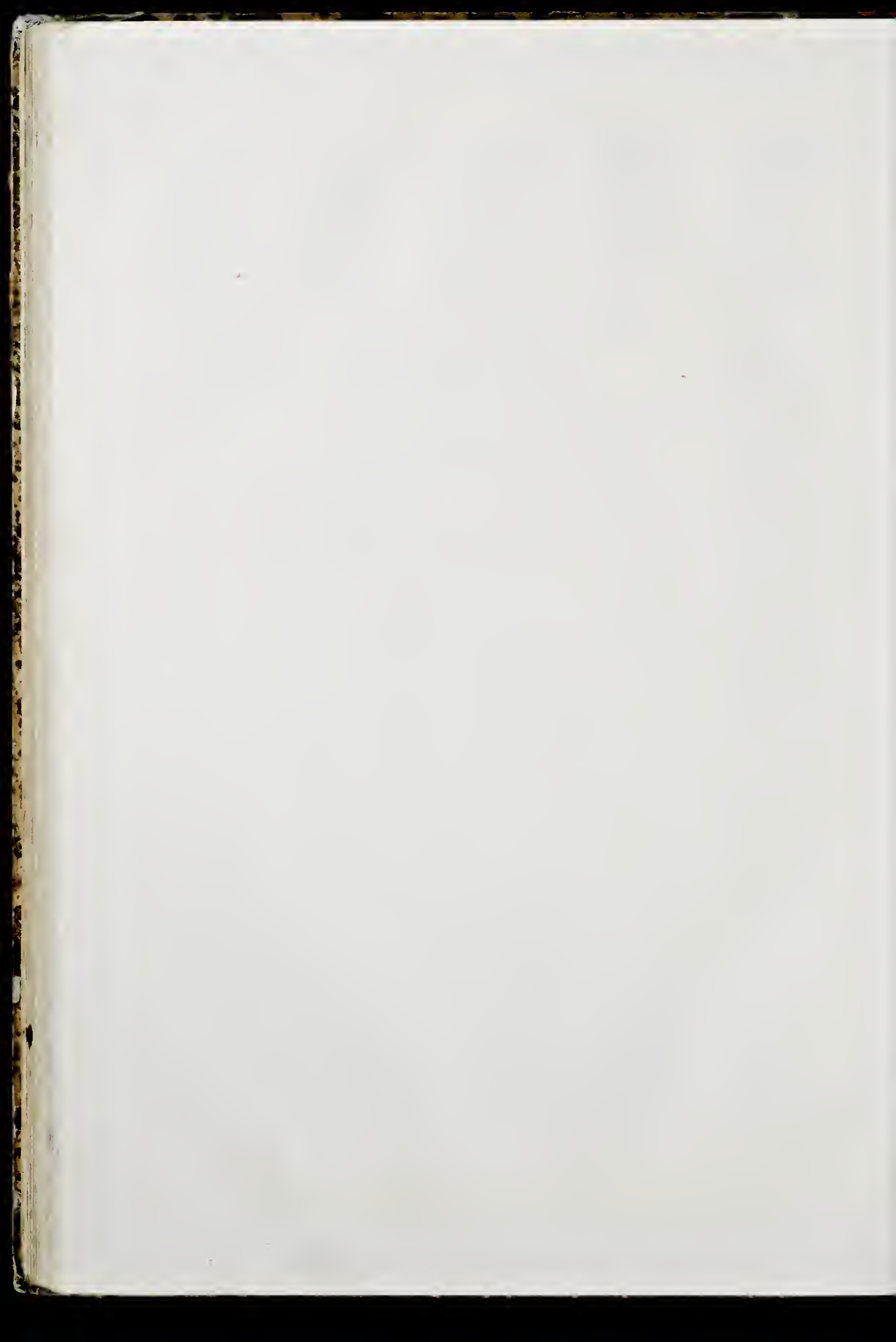




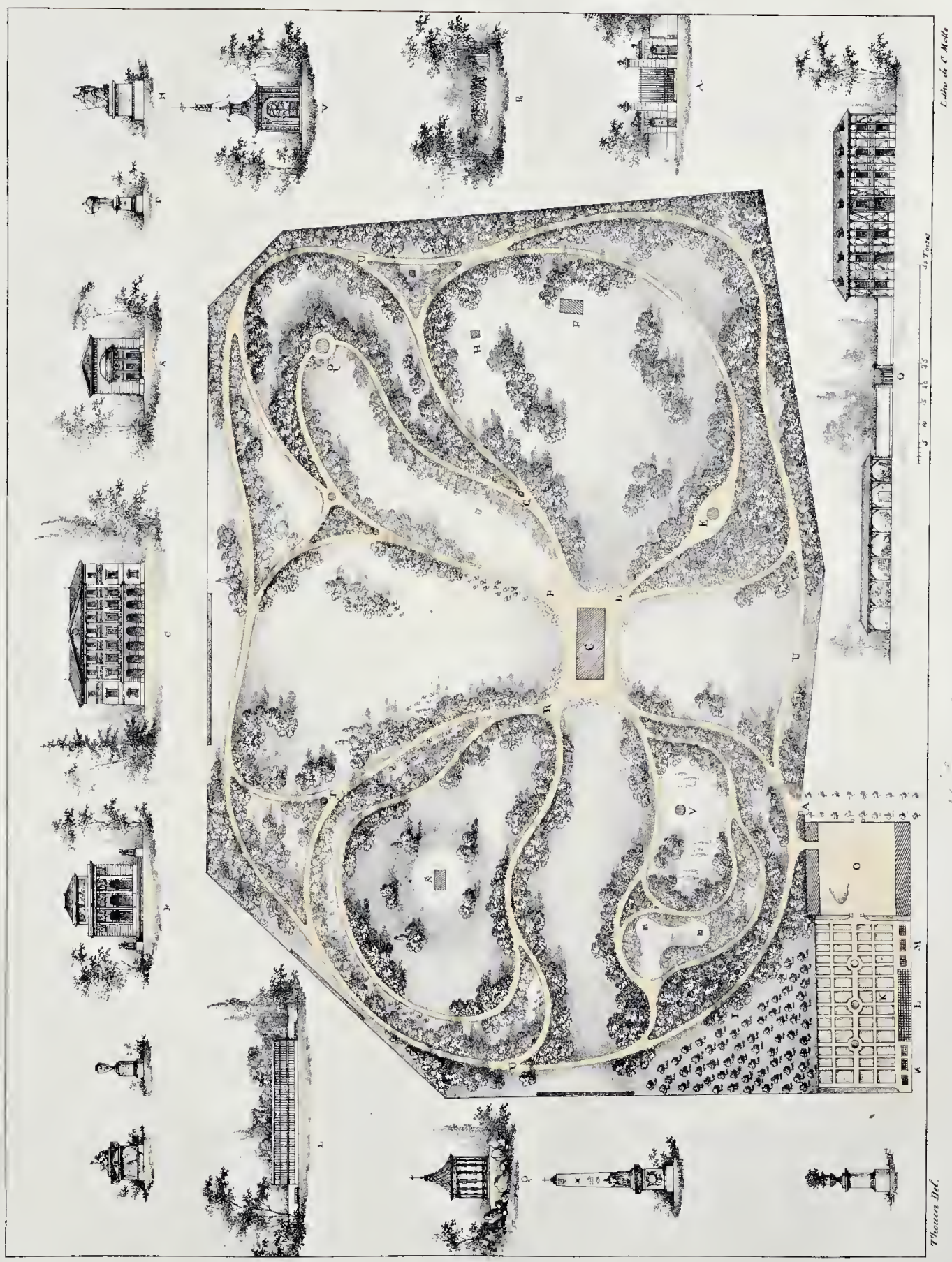




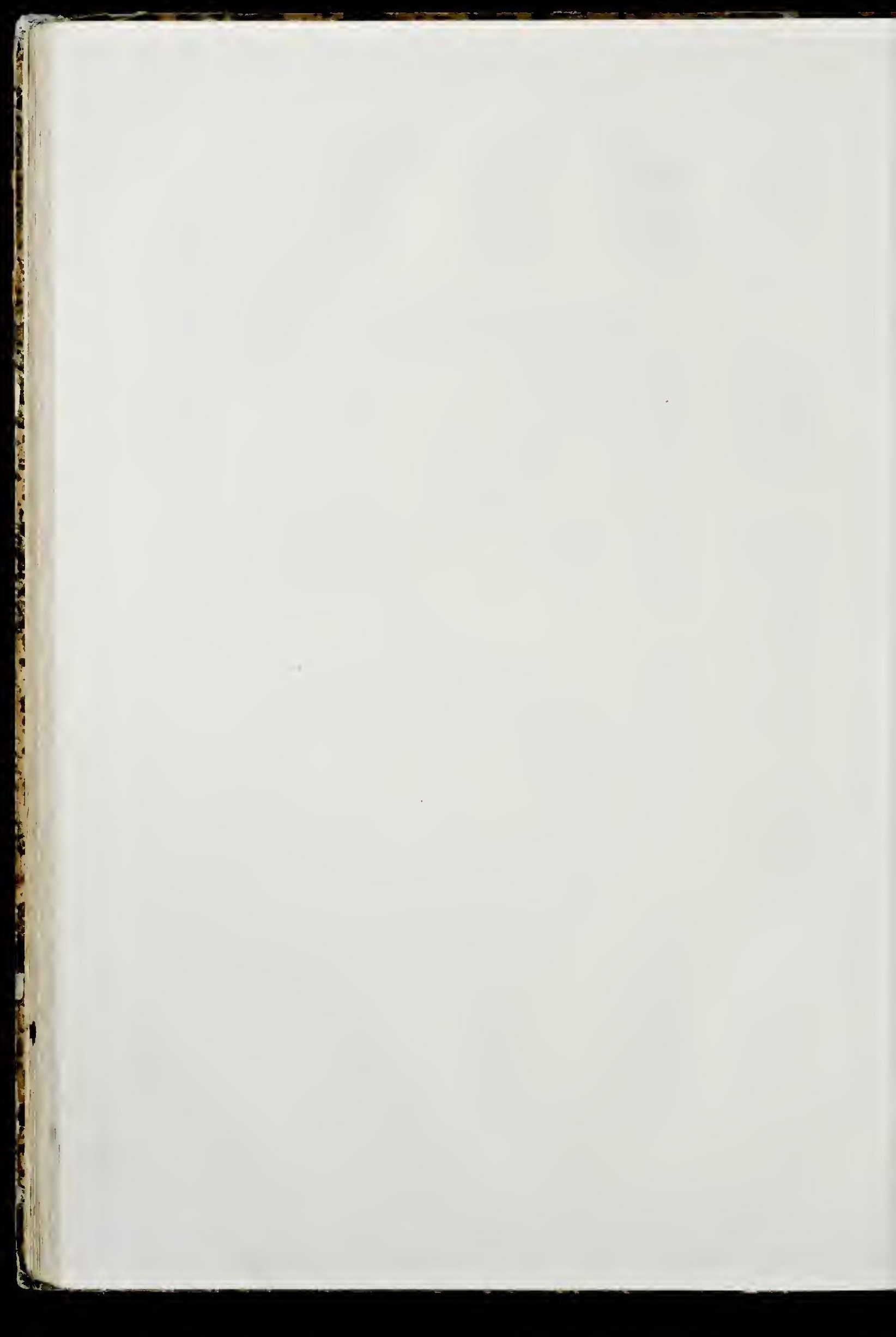




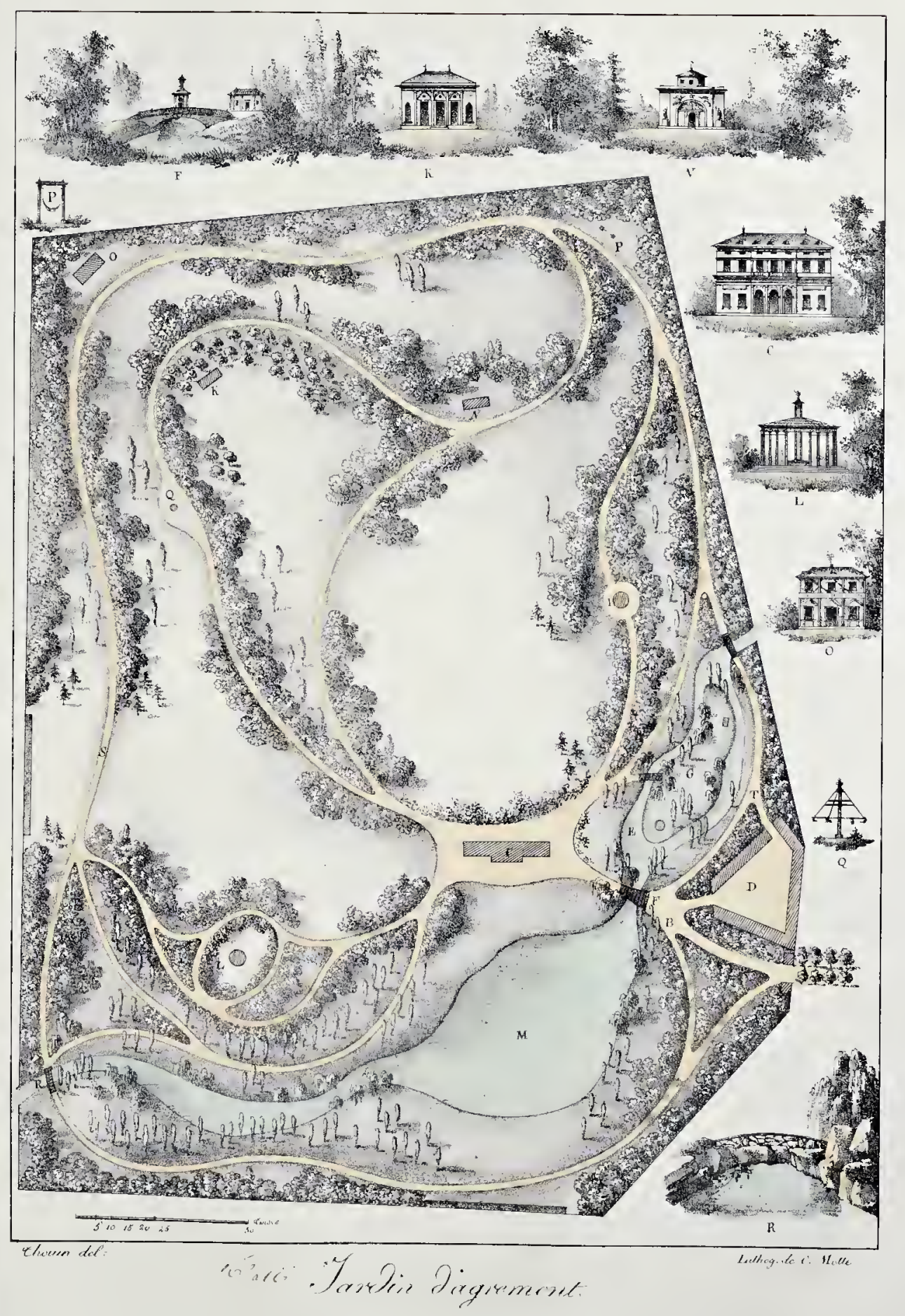




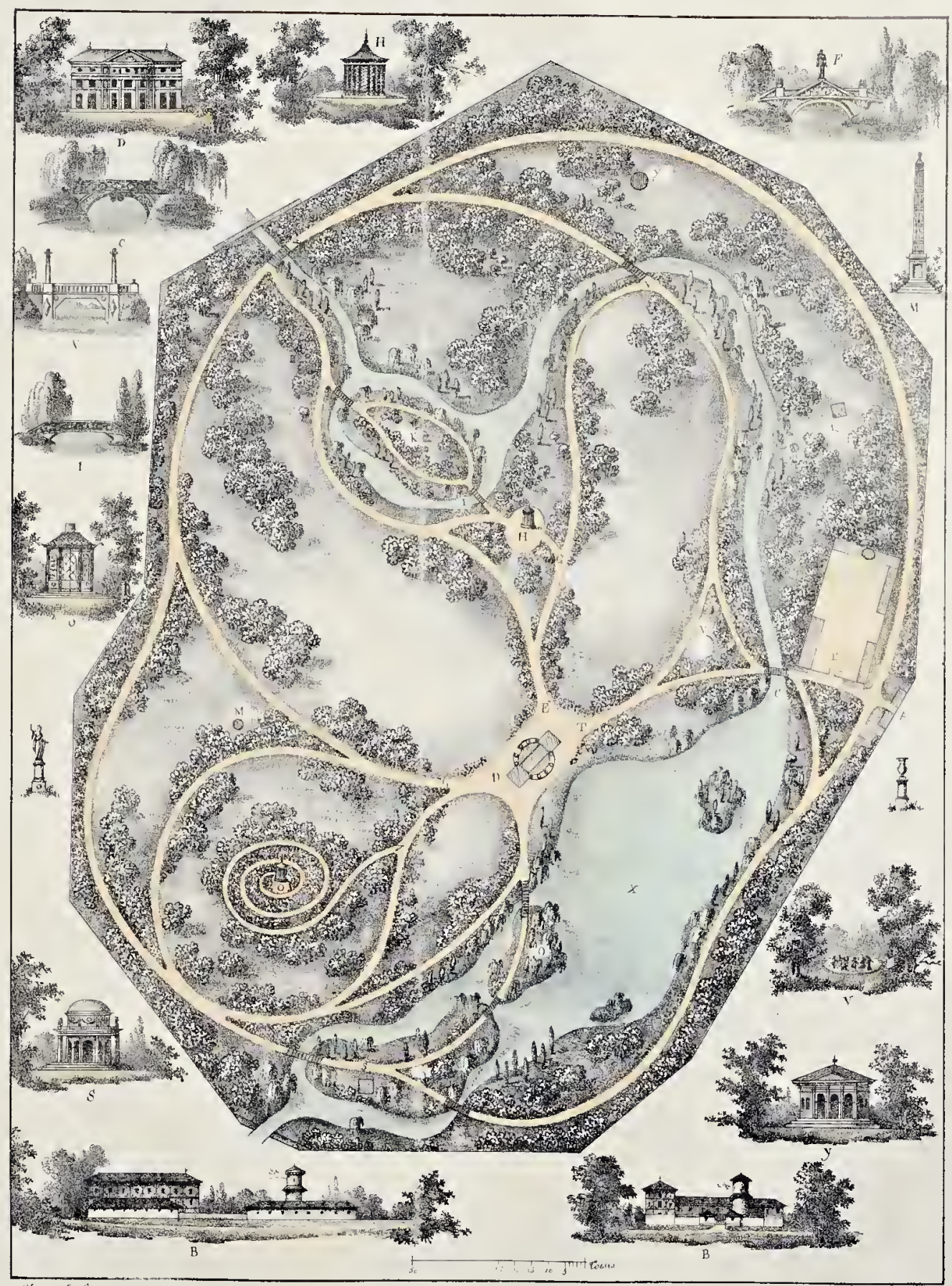

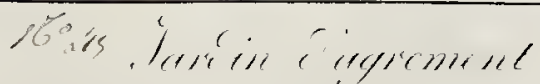

Lether de to theth 


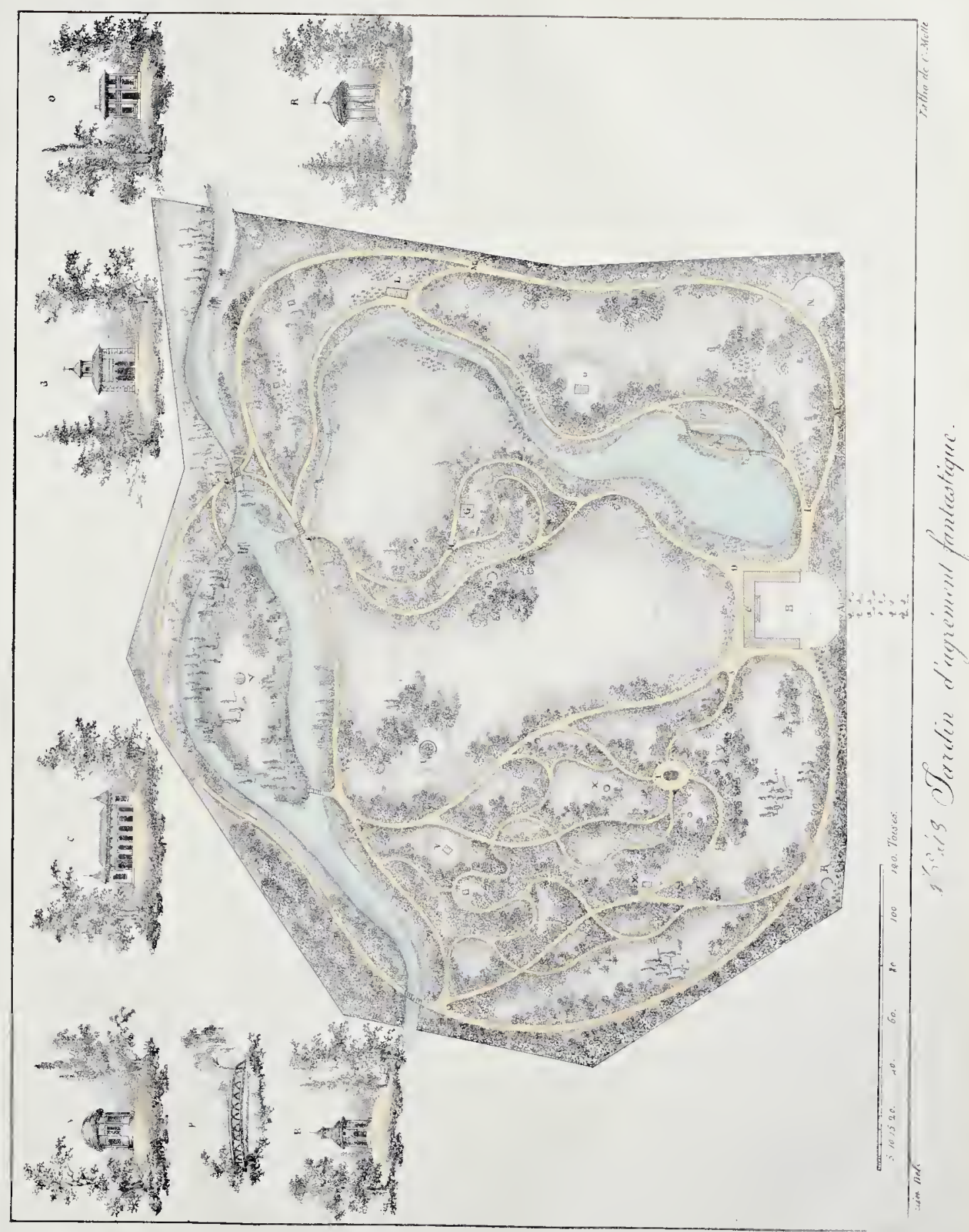




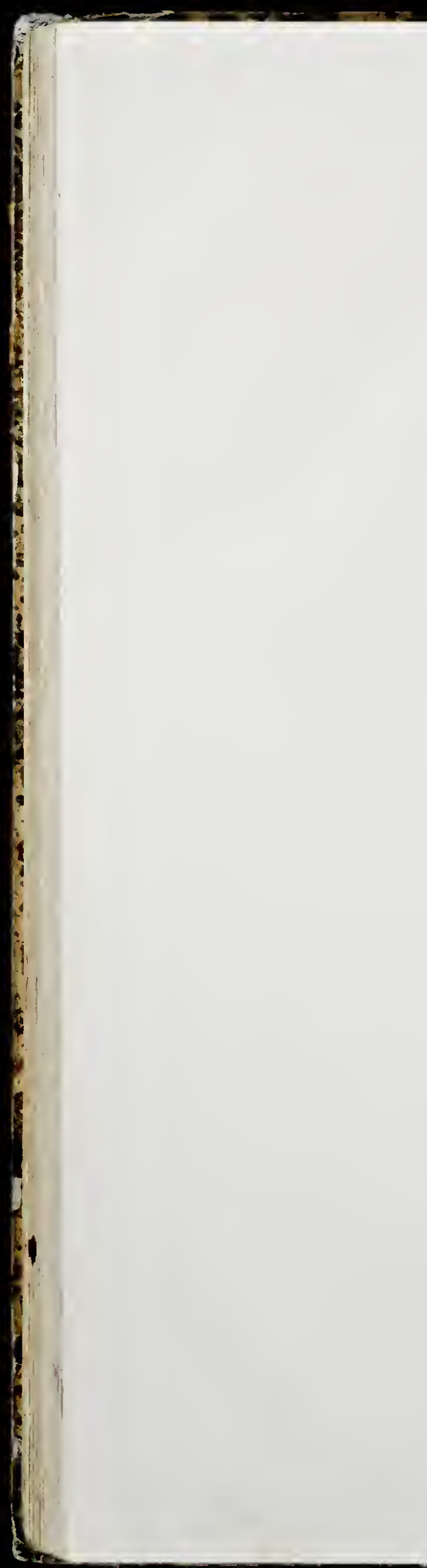




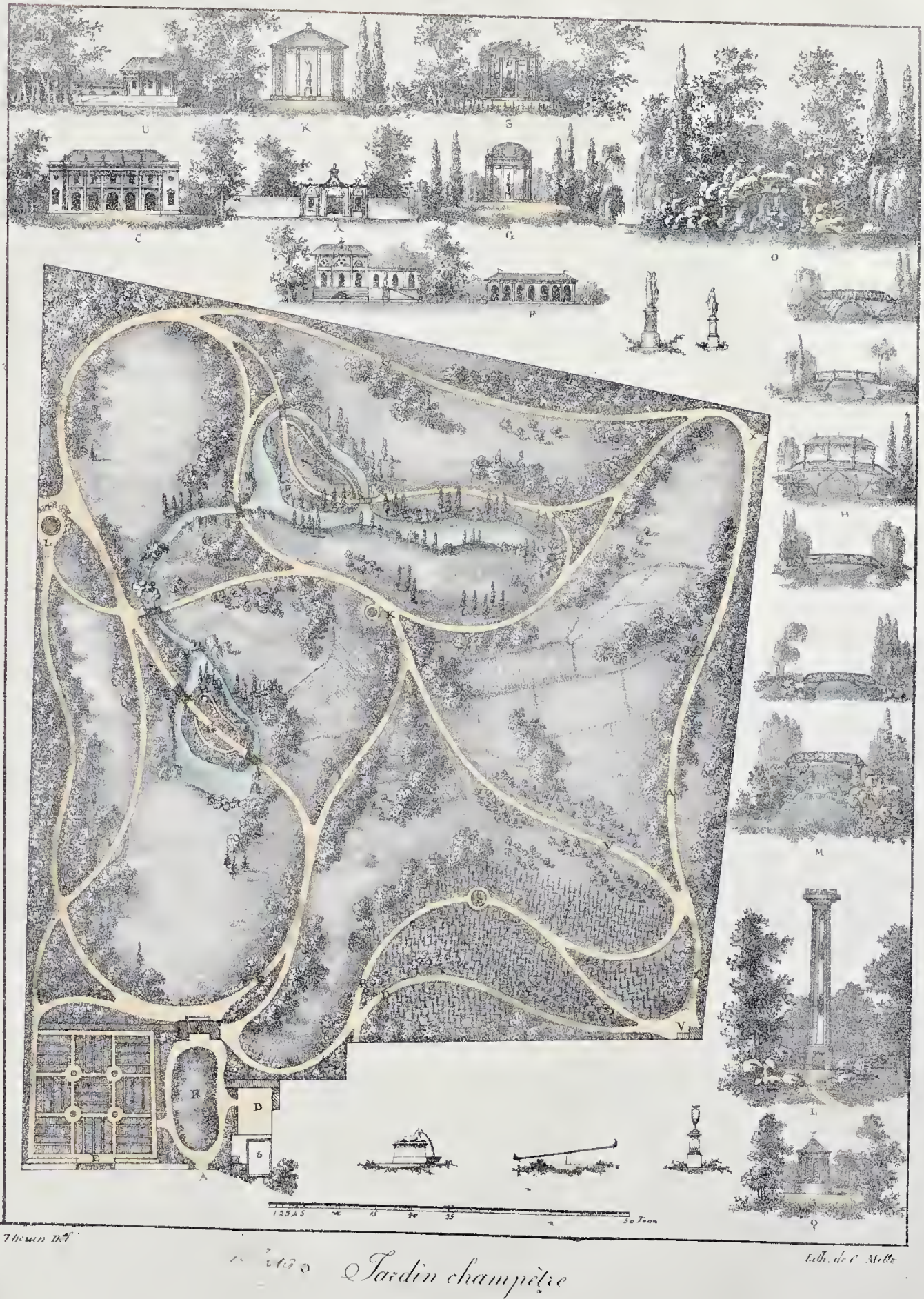




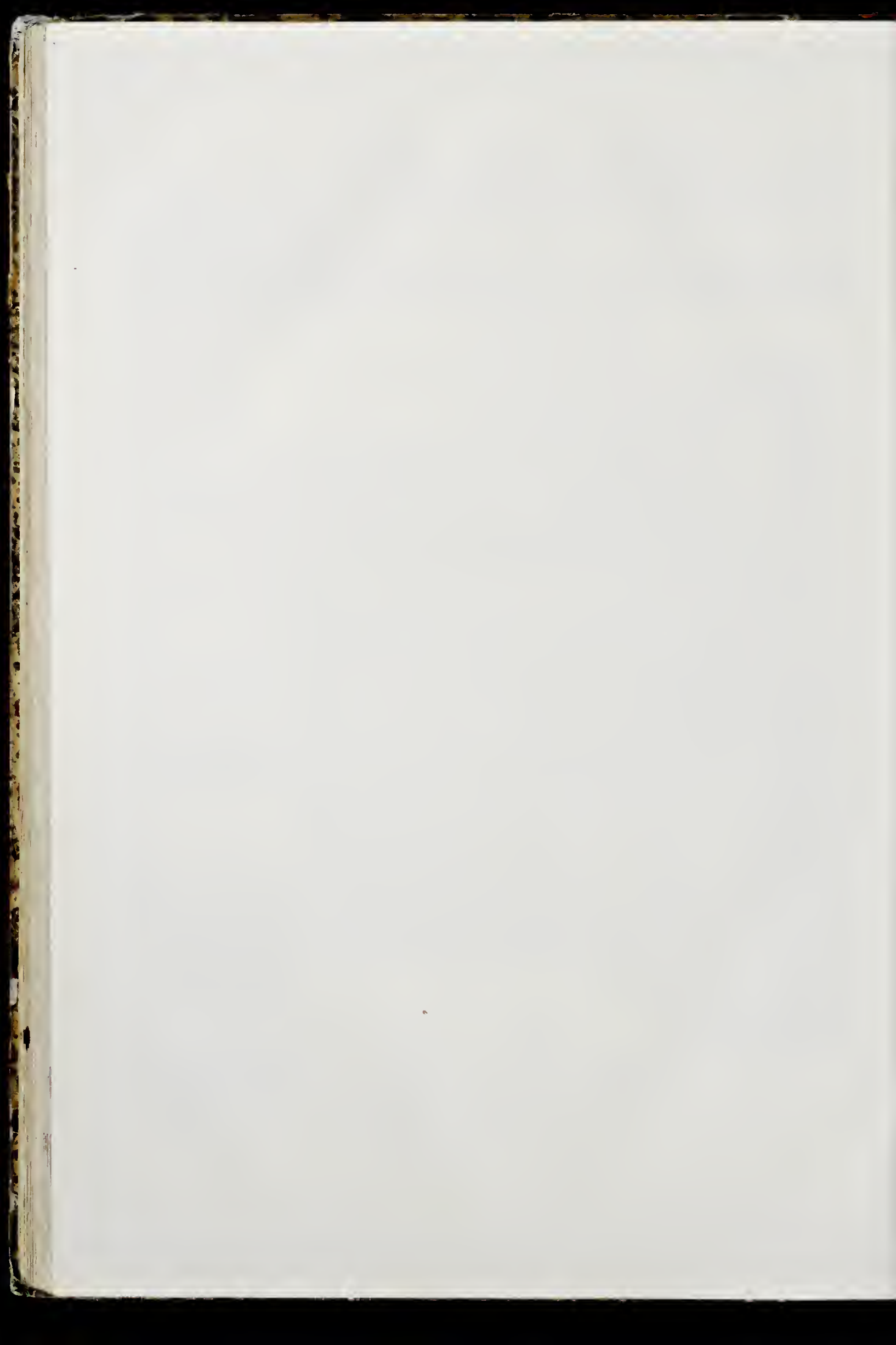




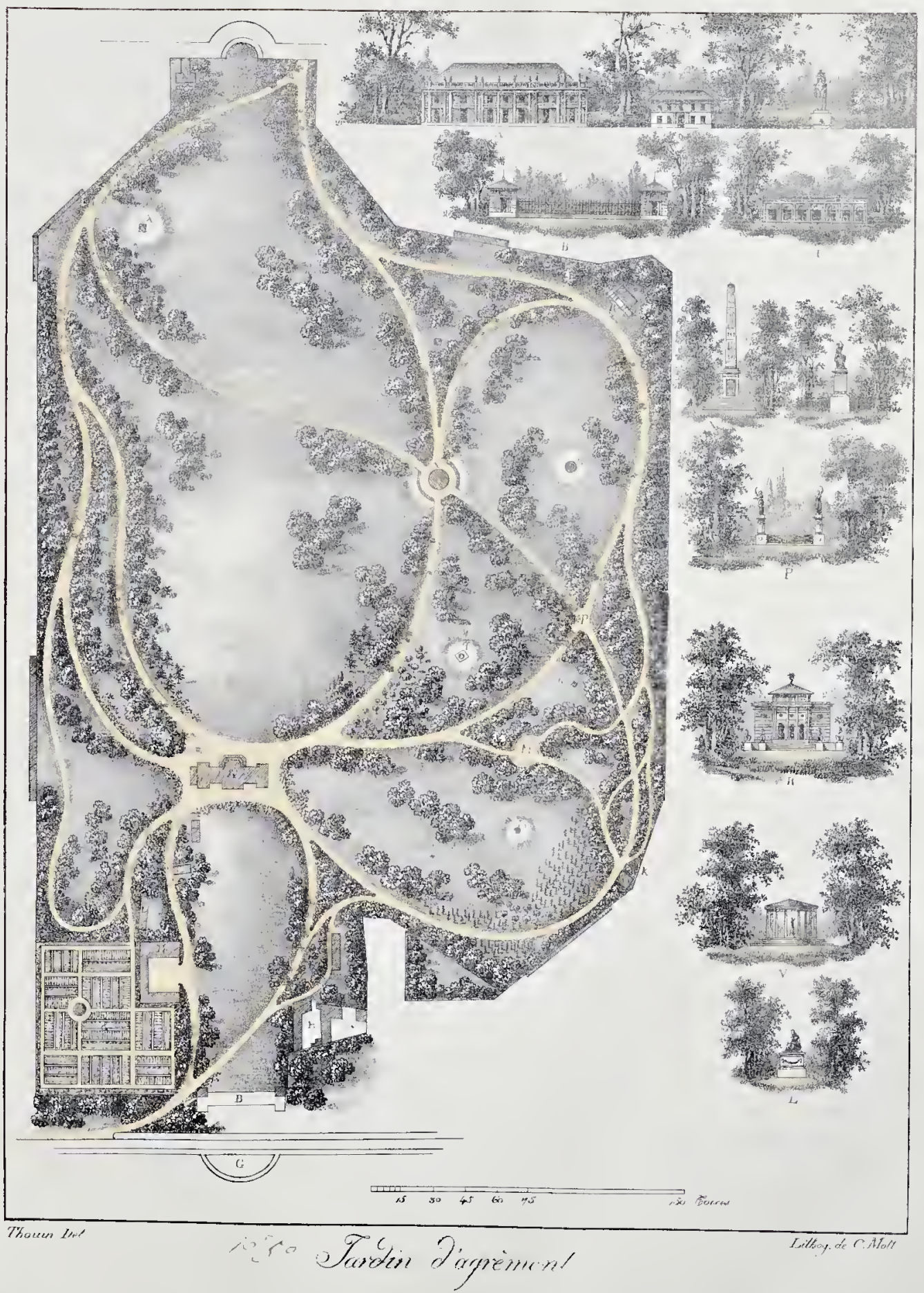




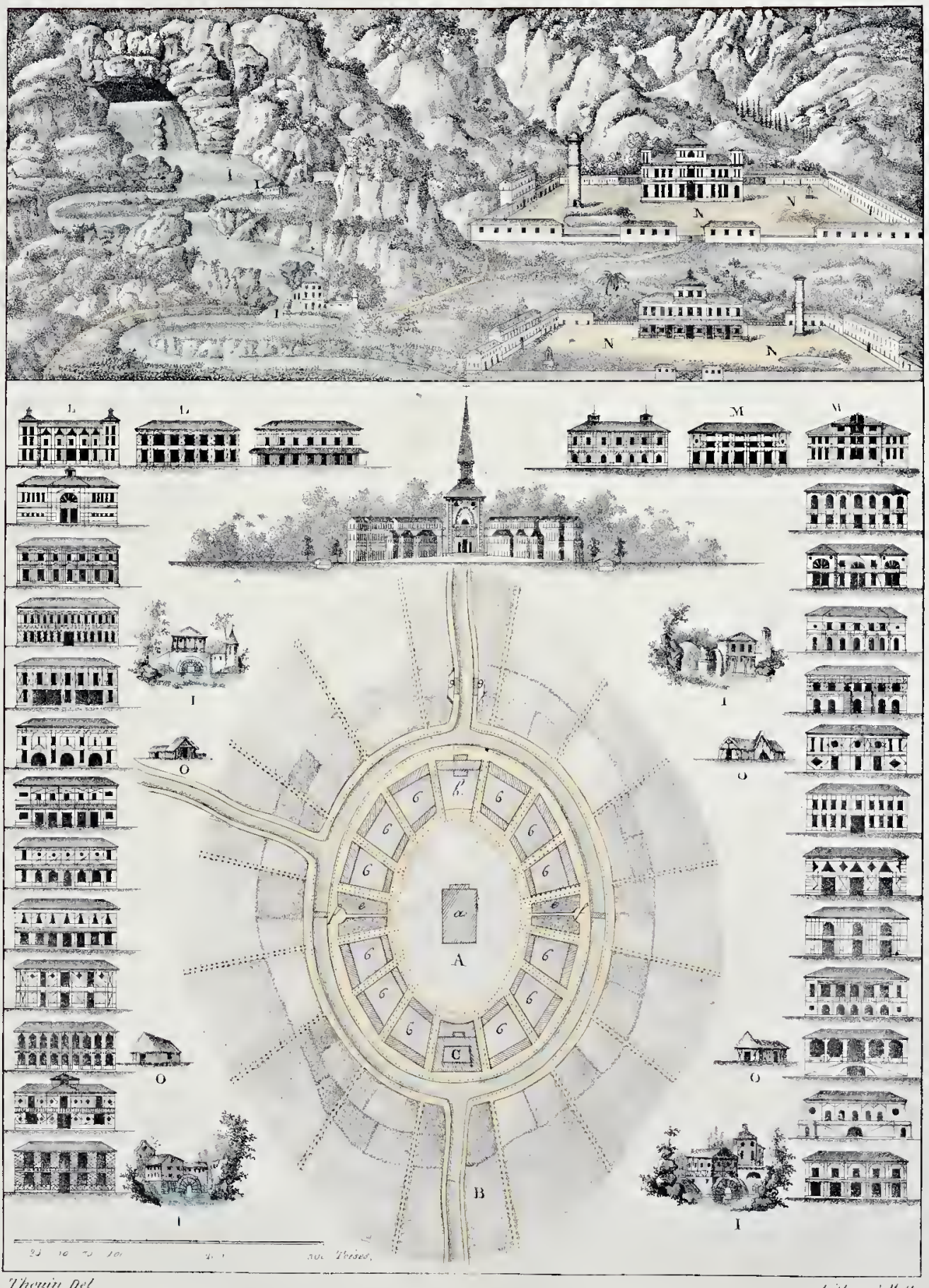

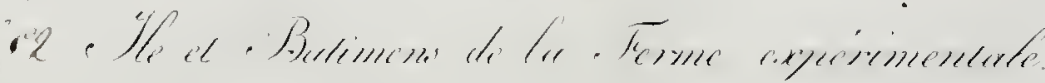




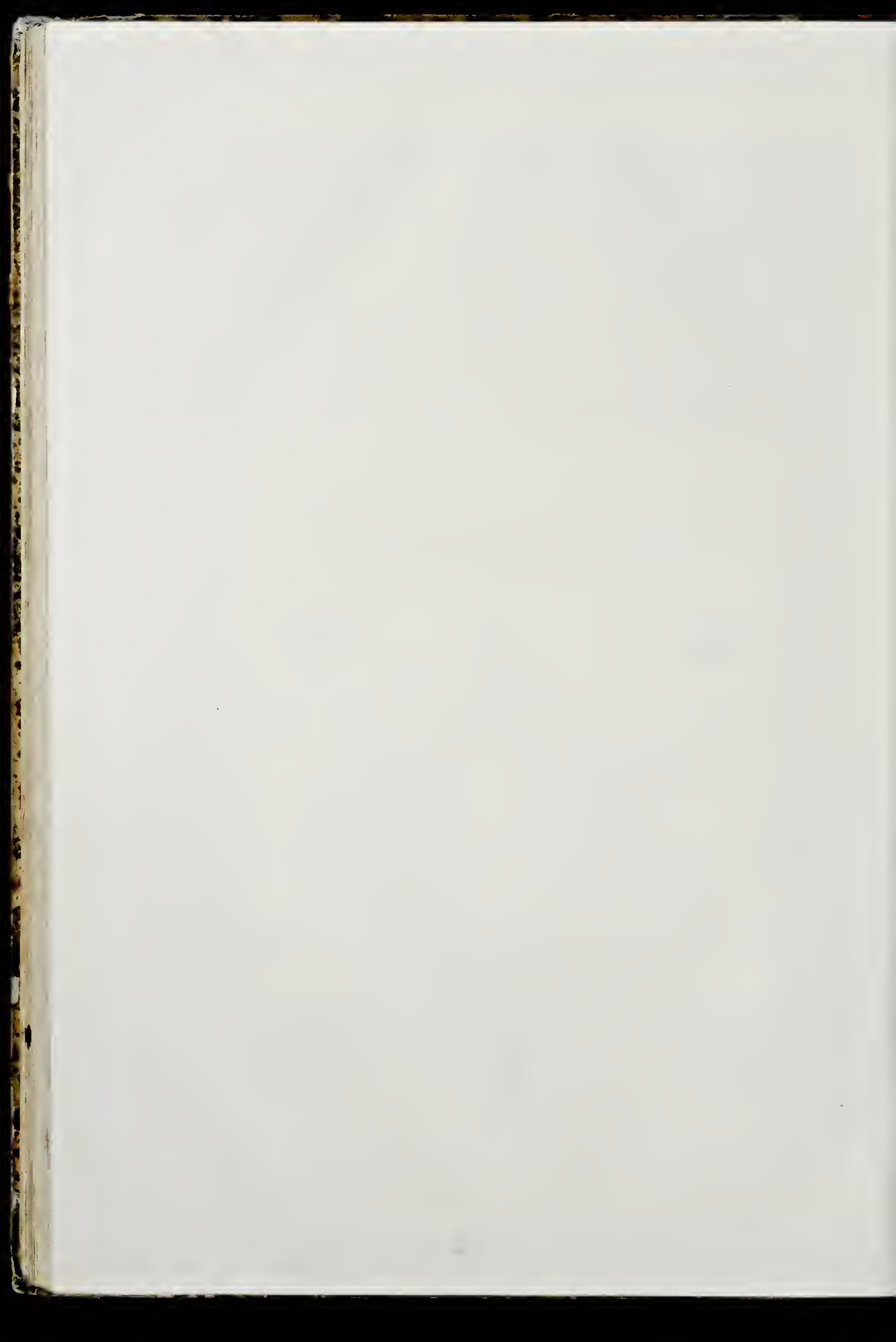




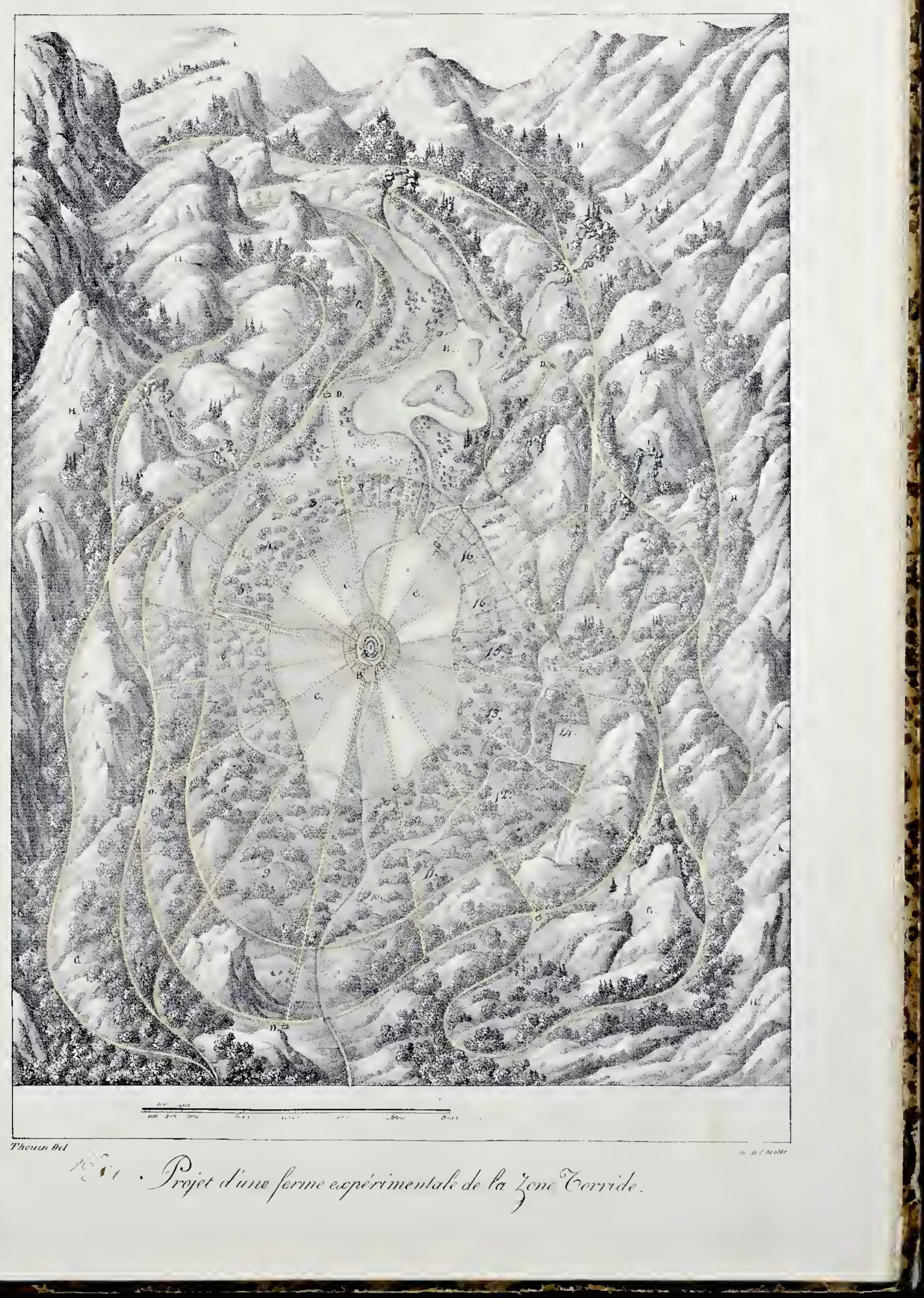




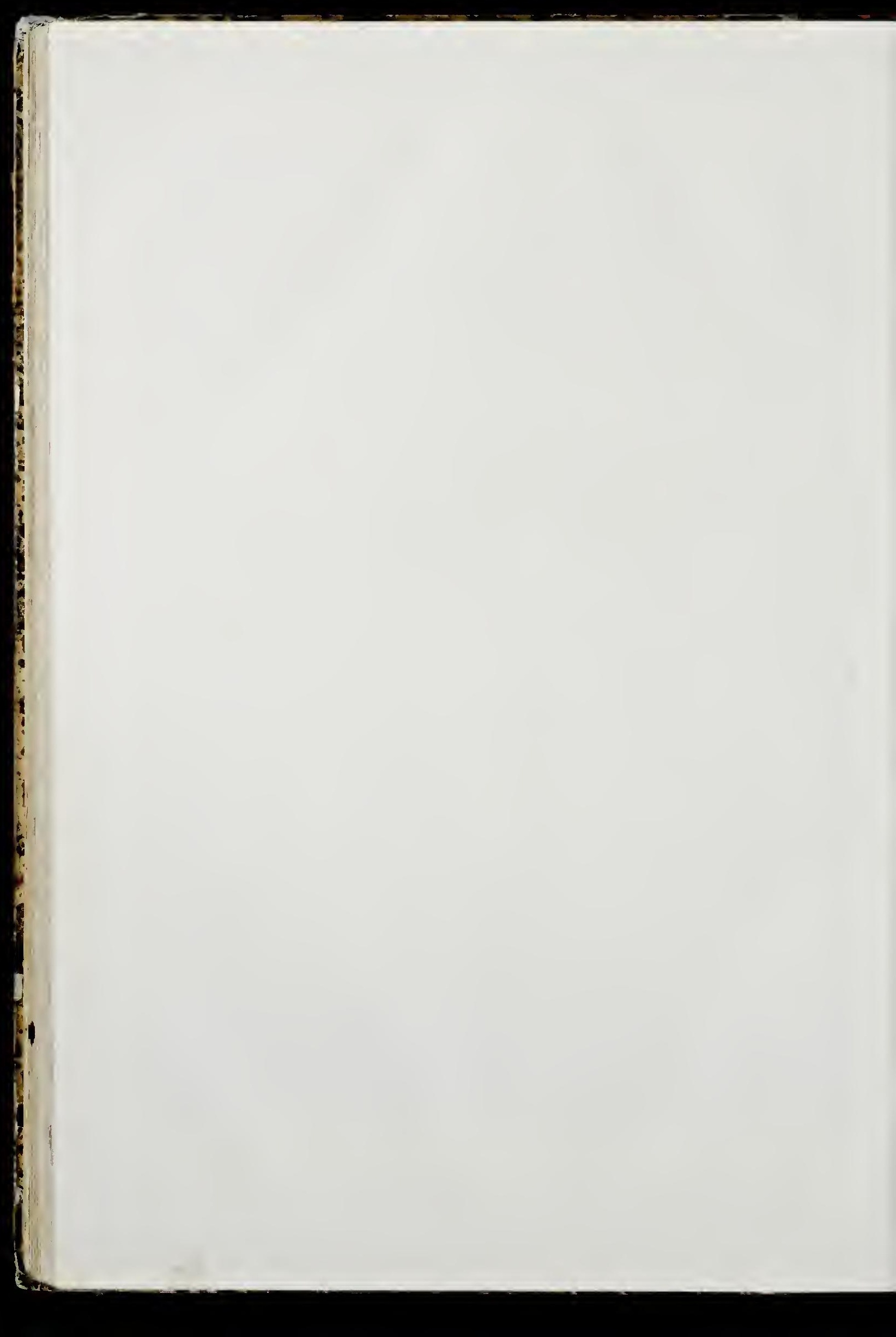



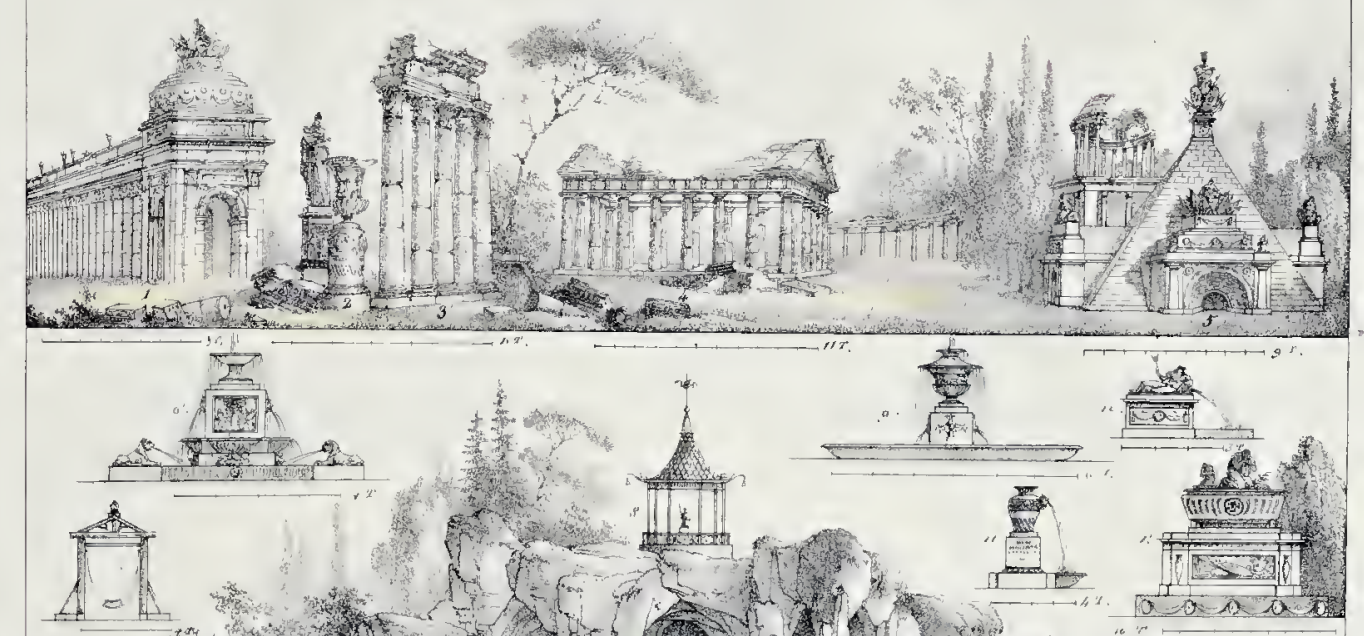

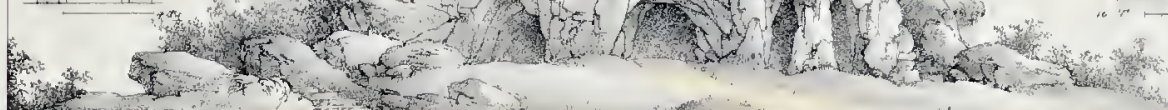

\section{tritstis

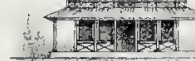

$\begin{array}{r}r+2 \\ -1 \\ \hline\end{array}$

$8+y^{2}$
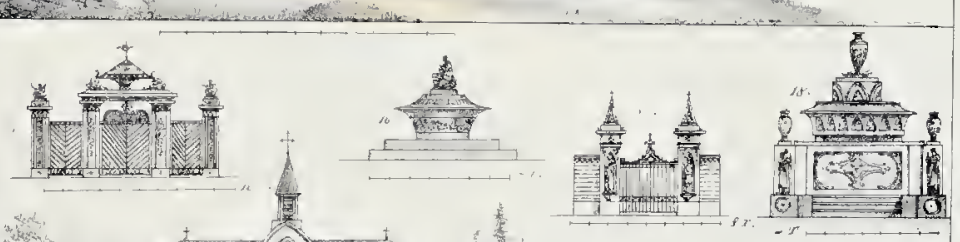

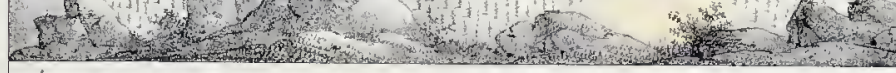
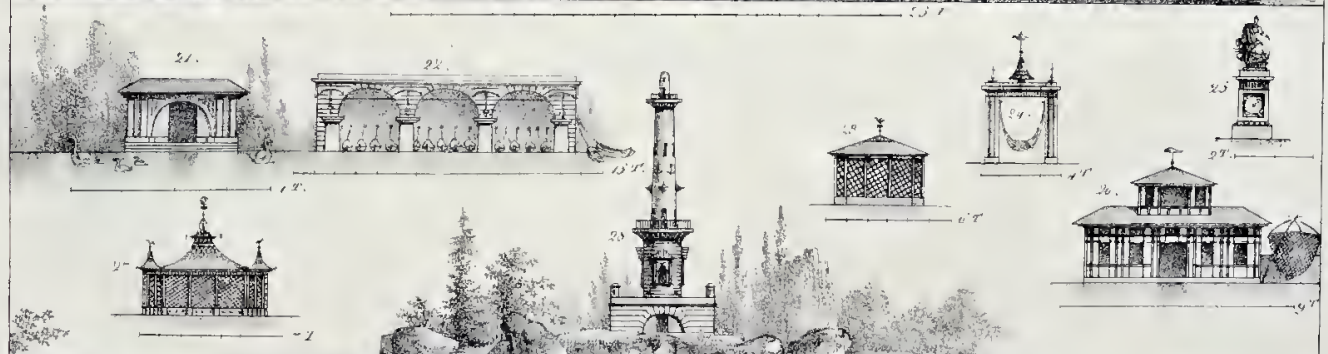

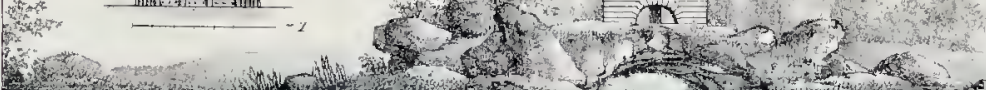

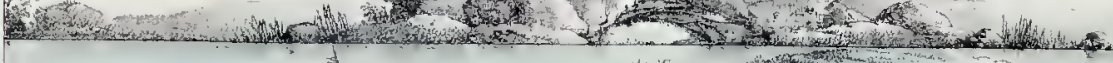

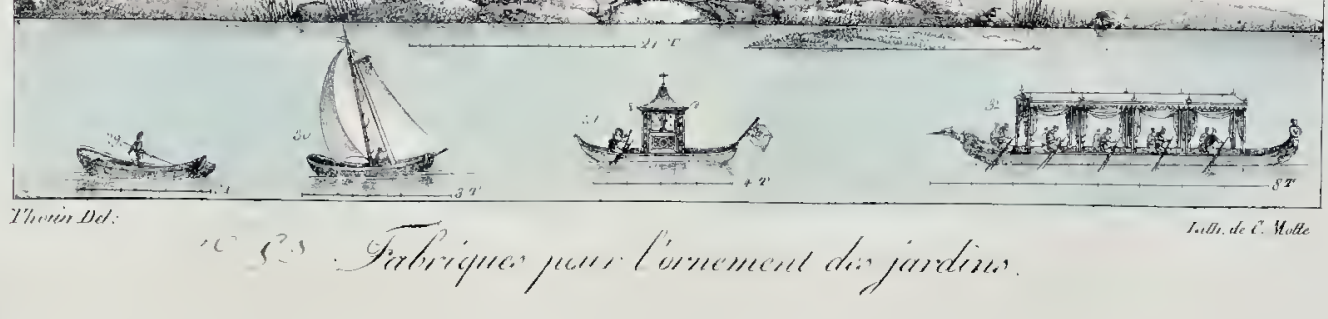




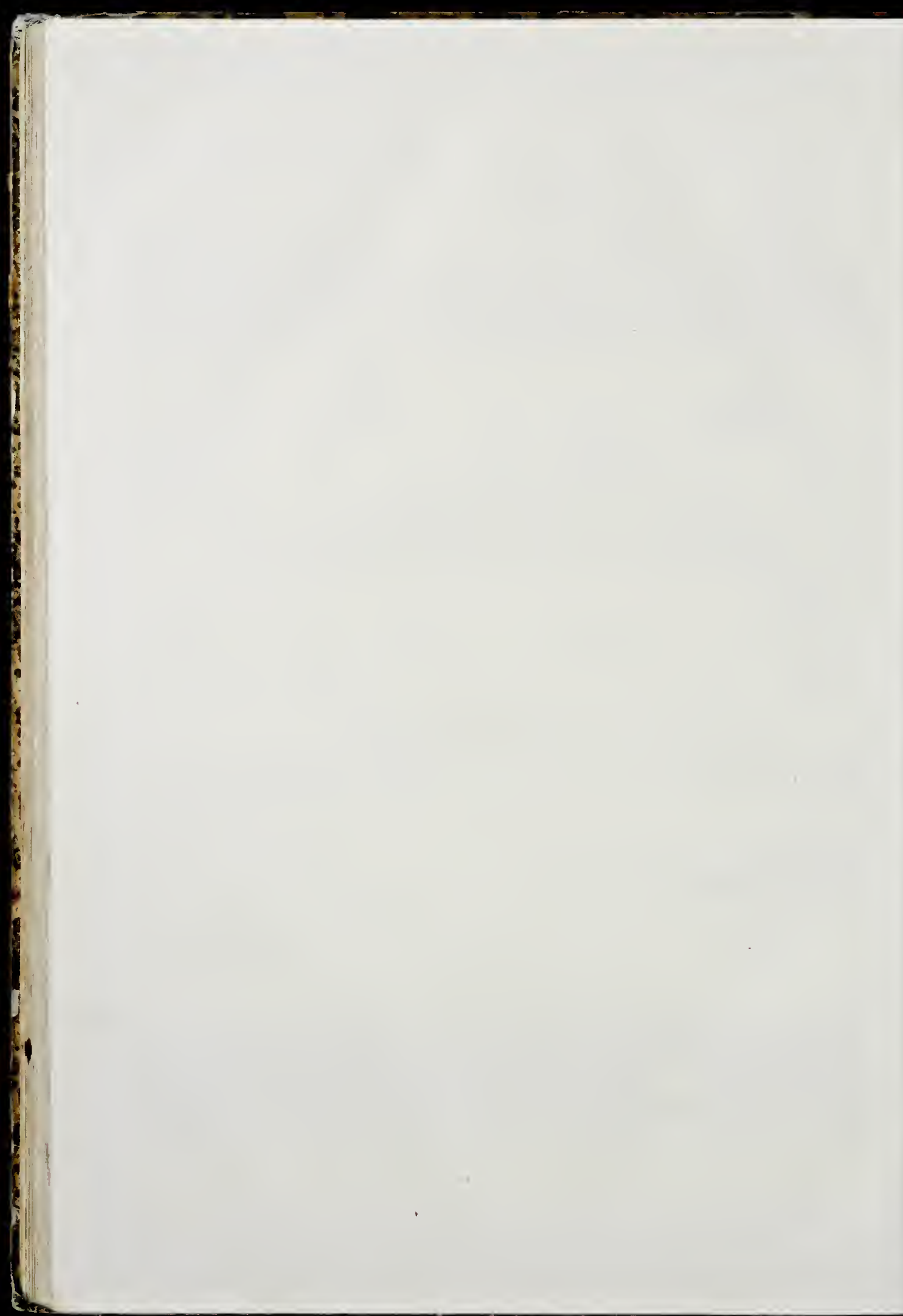




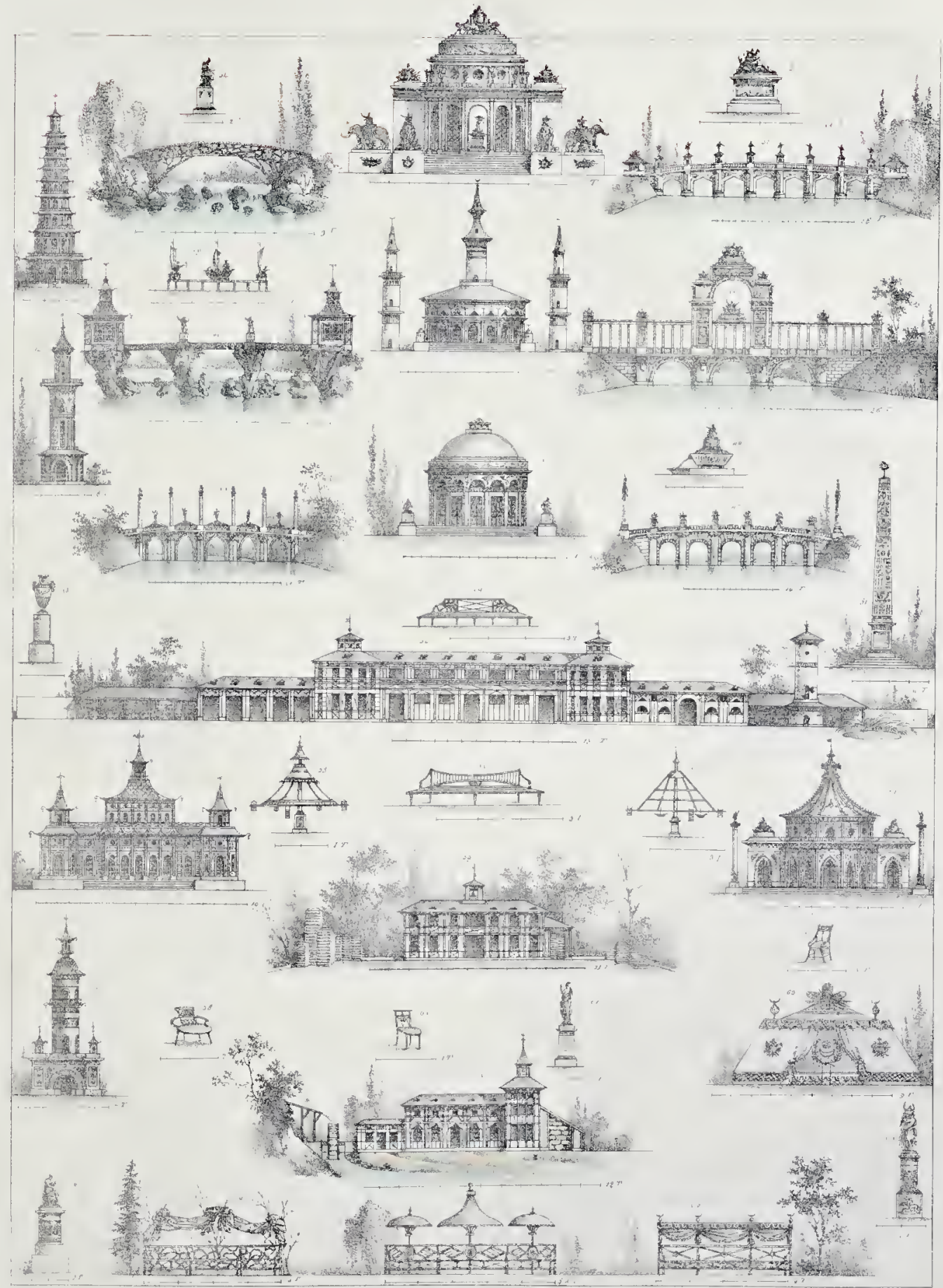




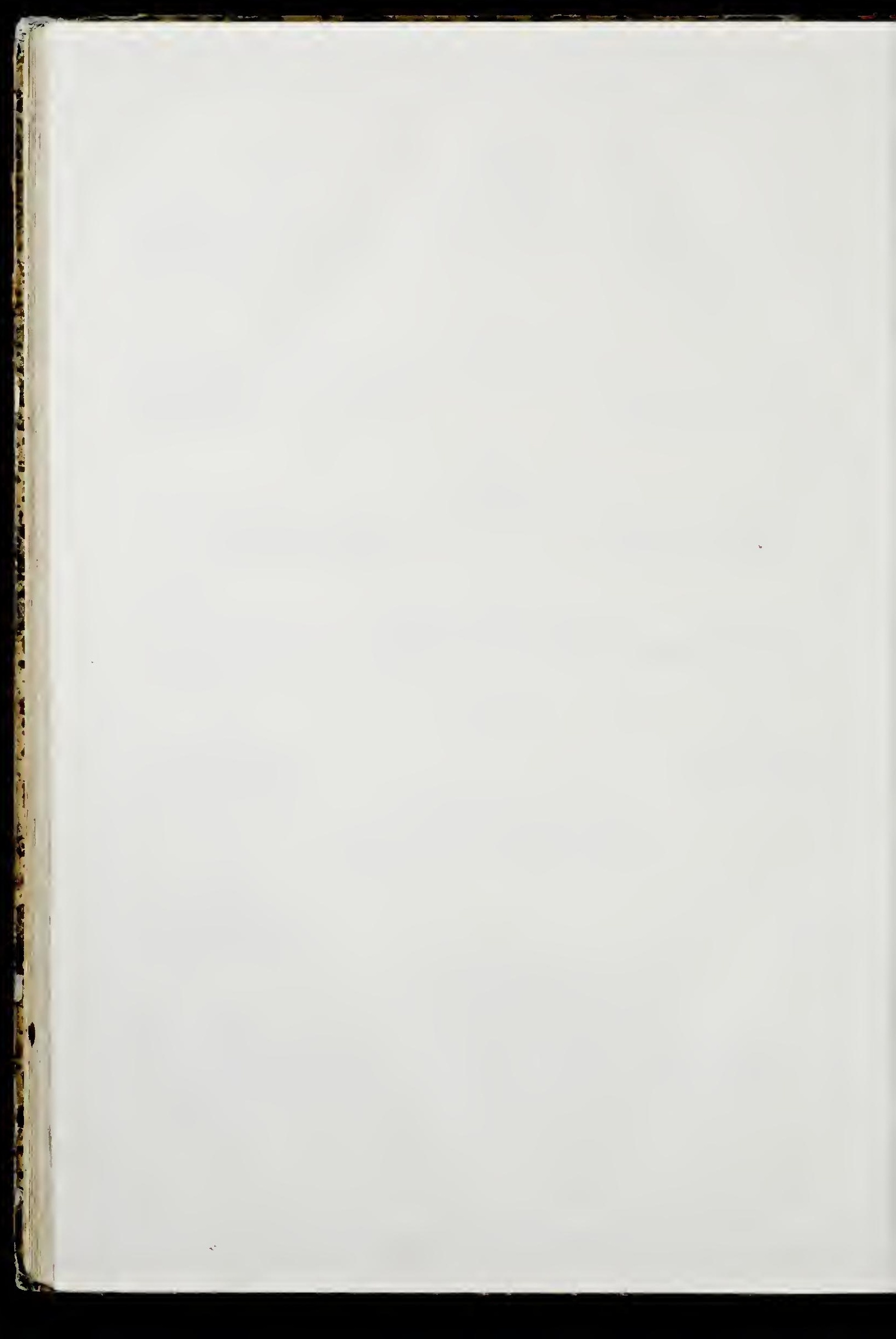




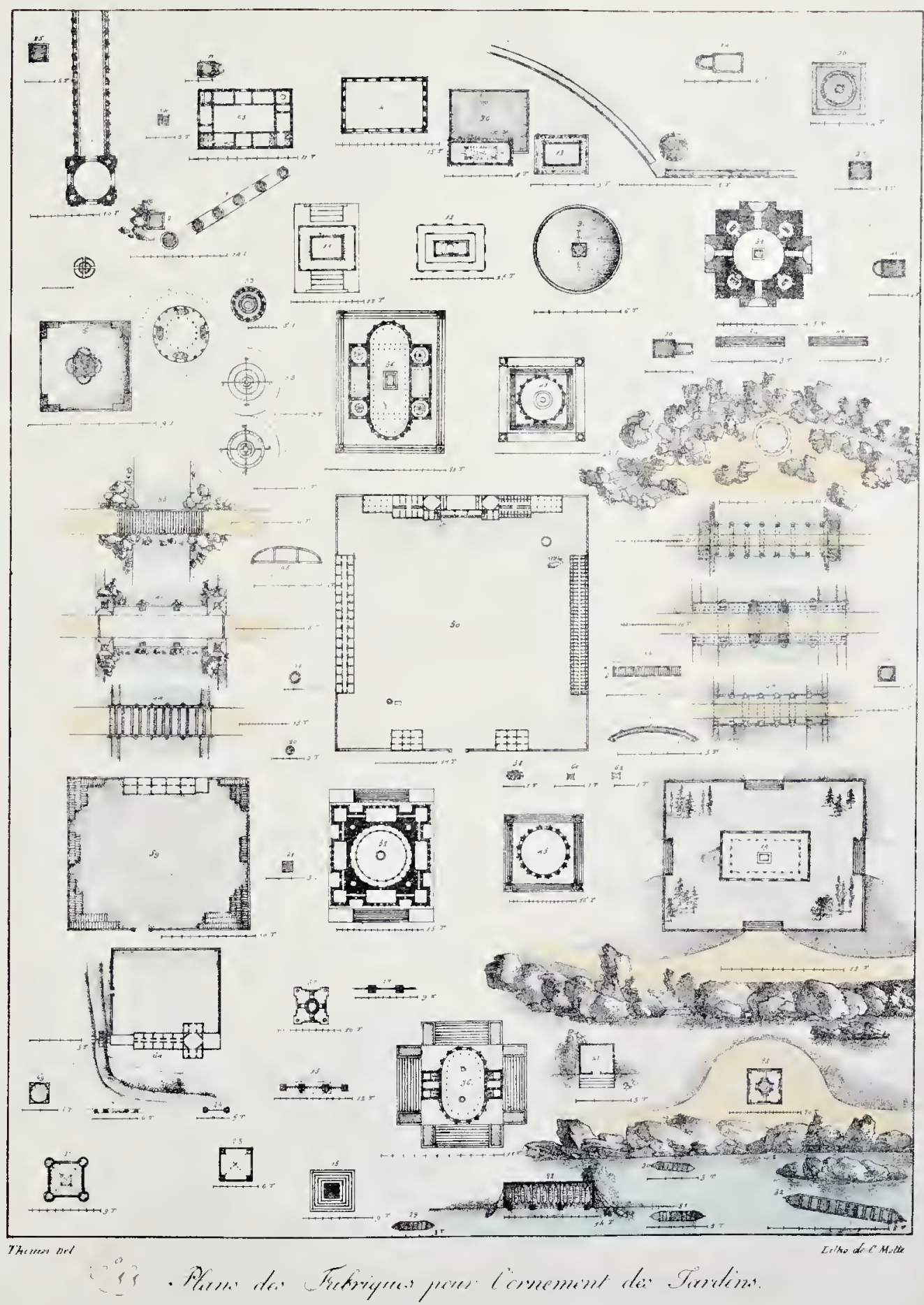




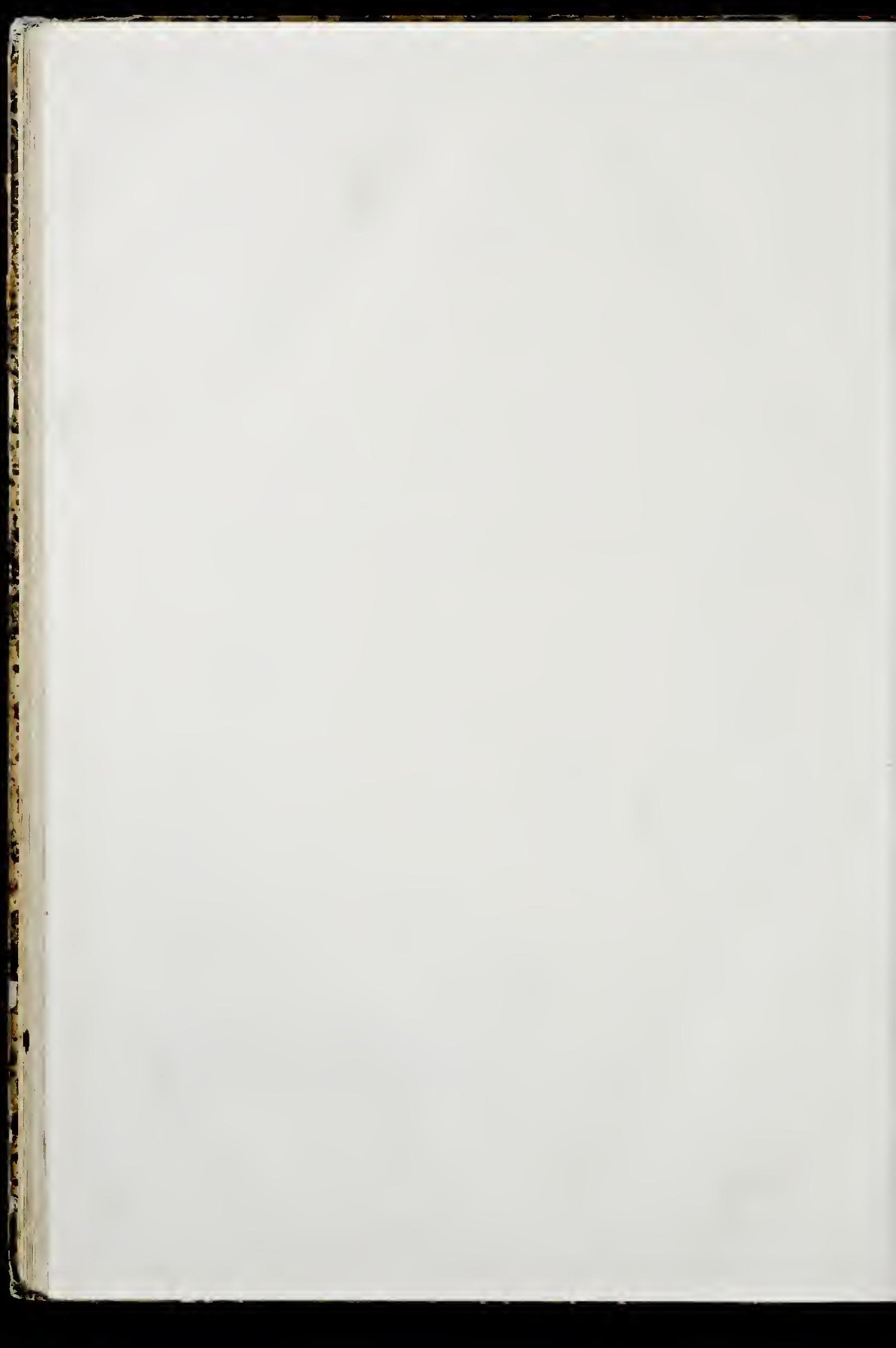




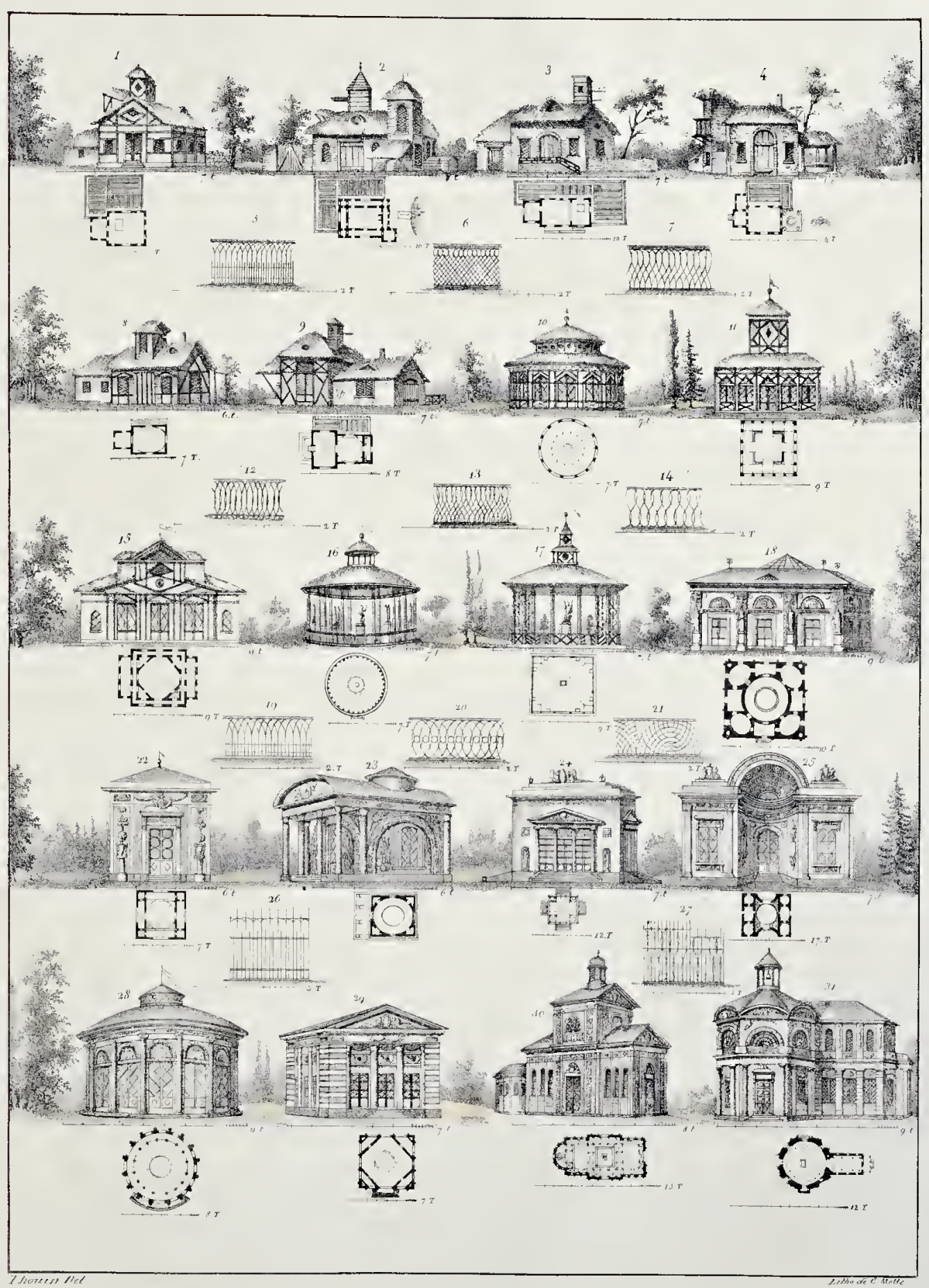




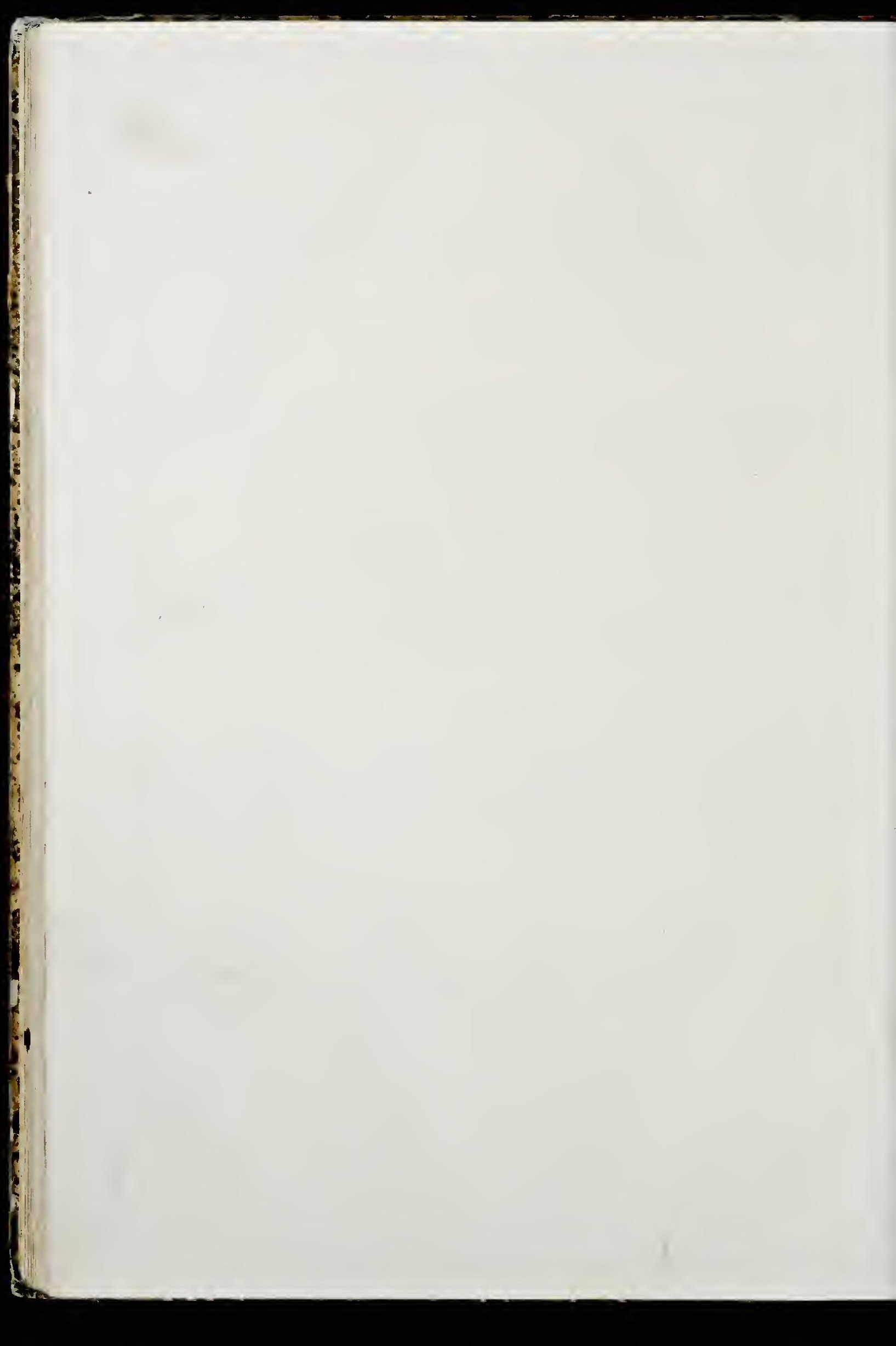





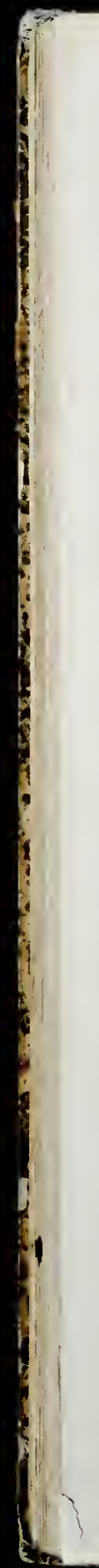




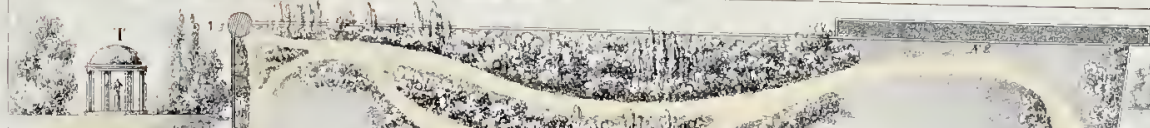

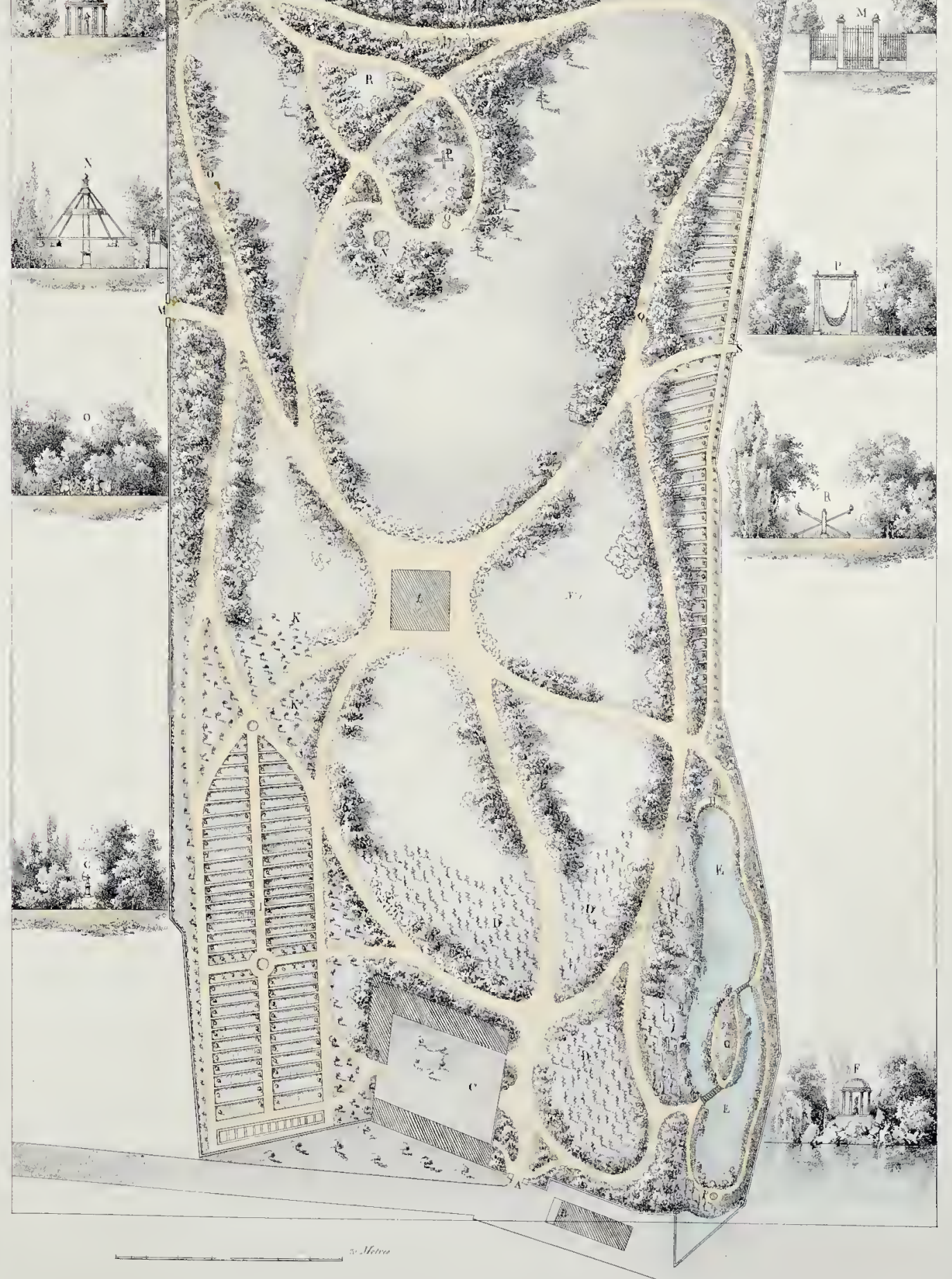




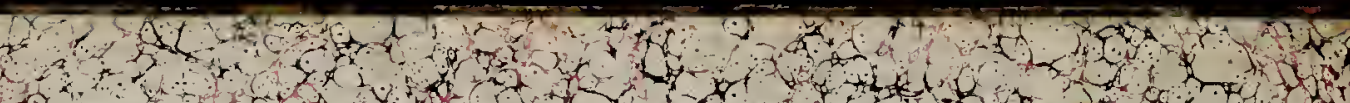

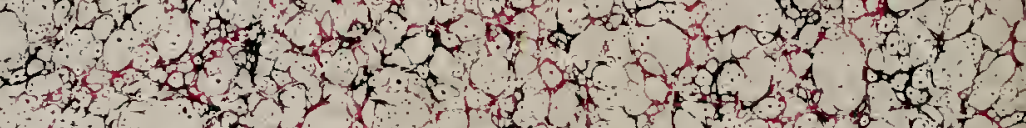

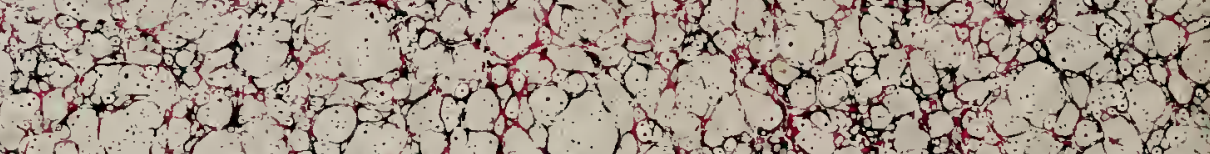

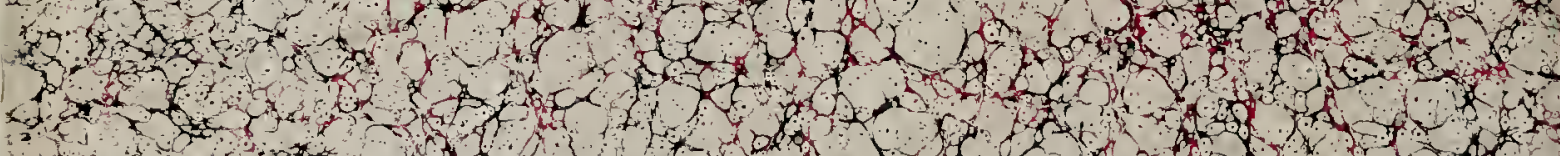

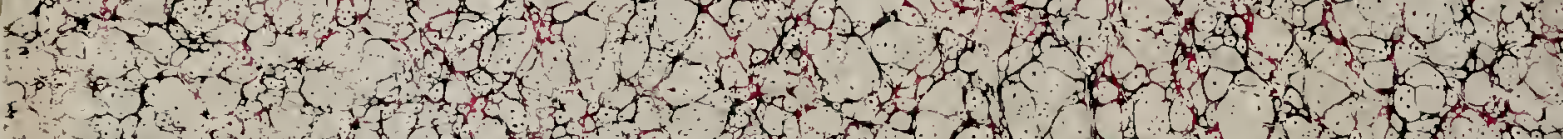

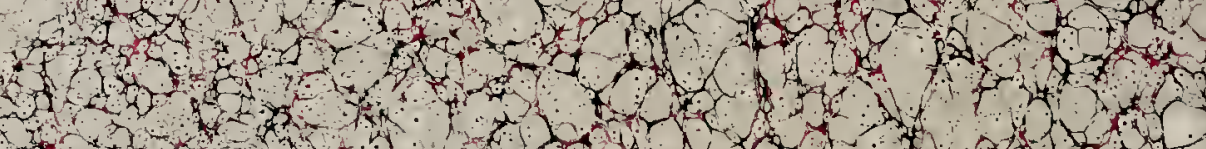
0.

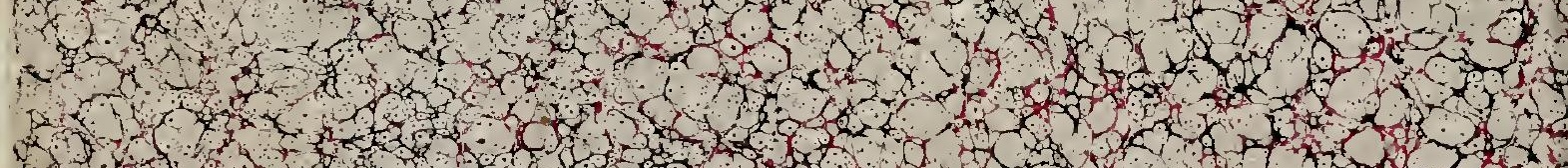

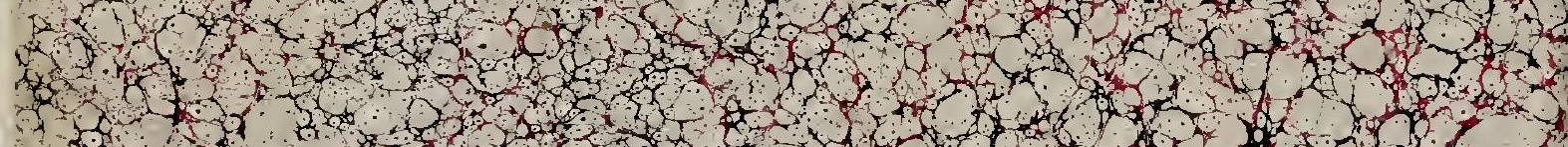

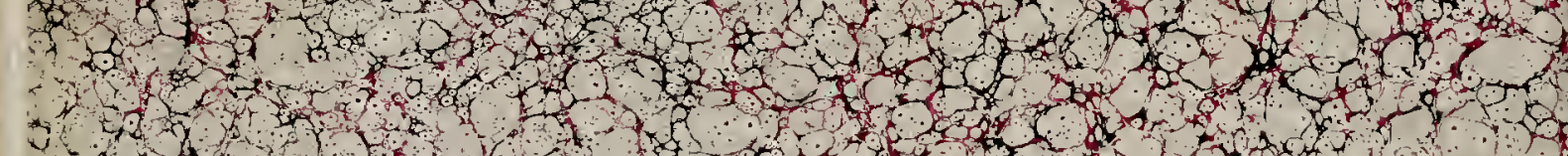

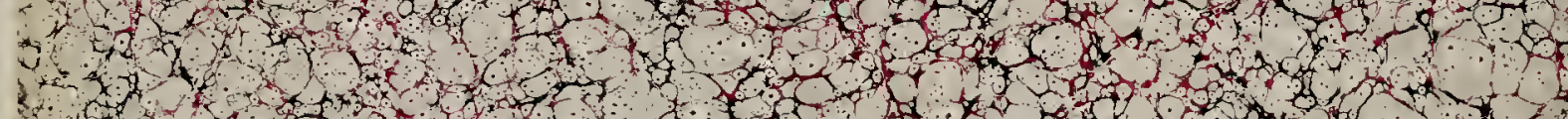

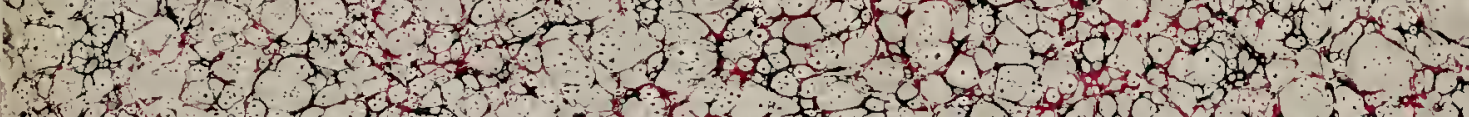

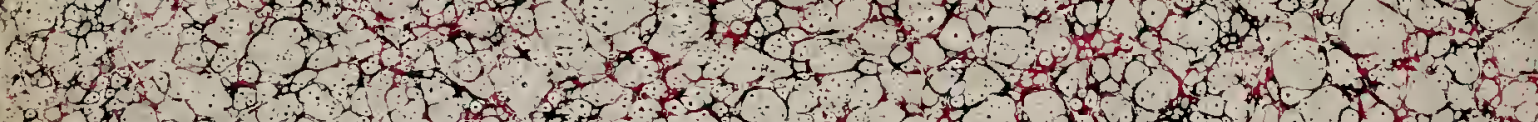

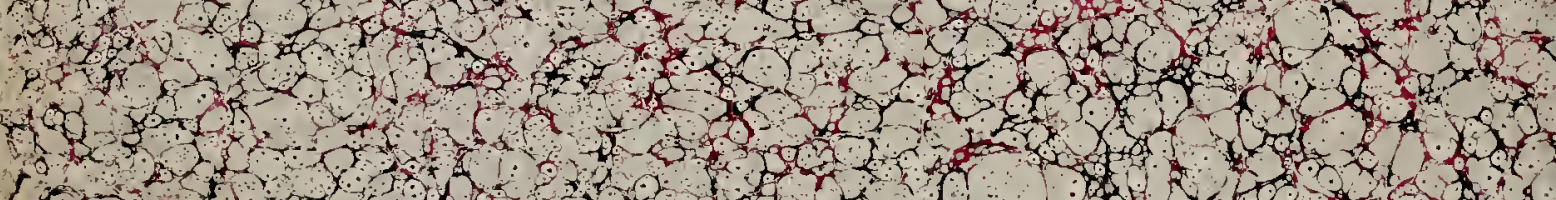

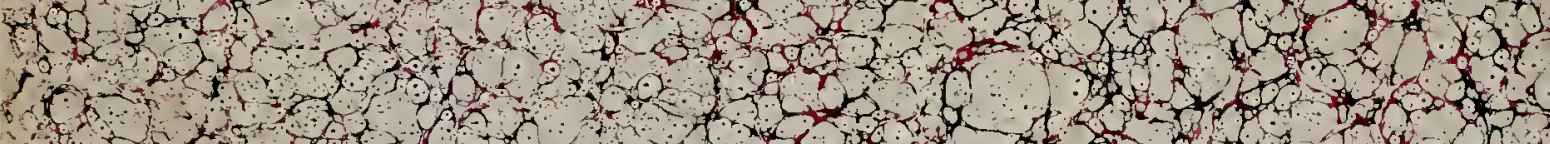

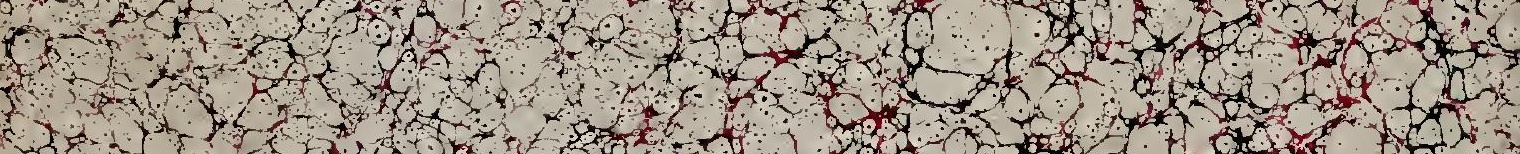

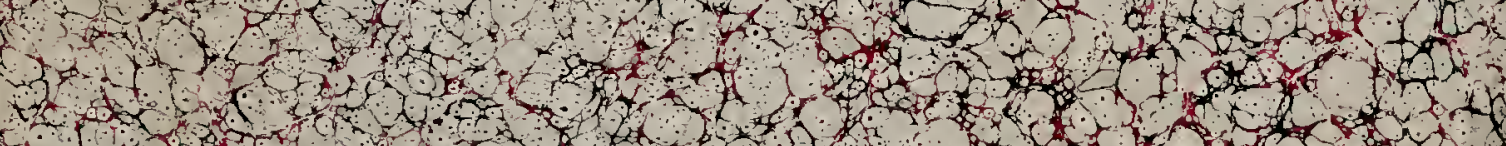

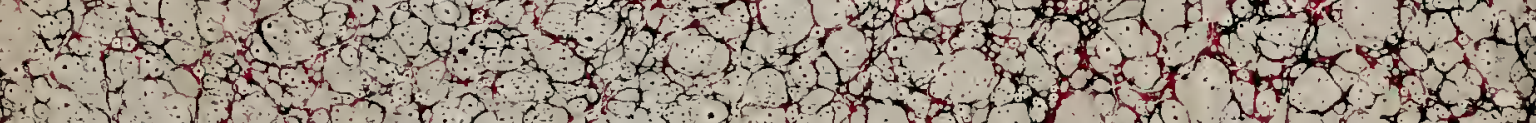
(5) 15x (5)

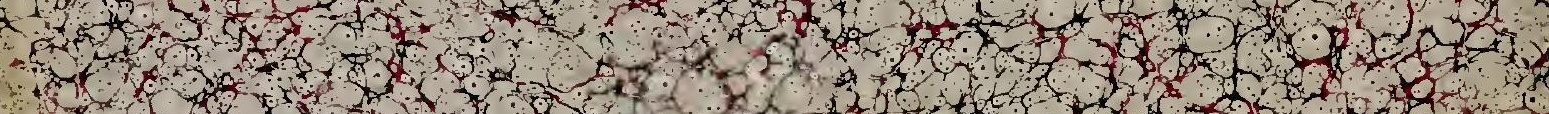

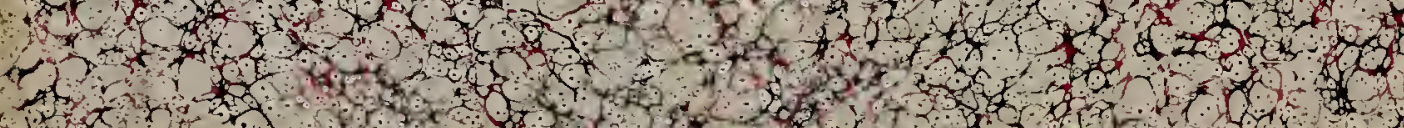




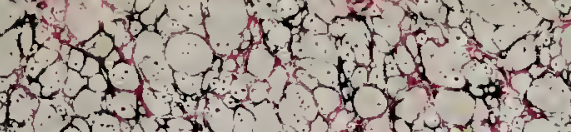

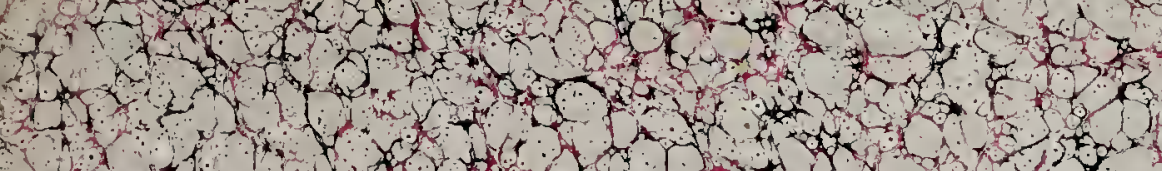

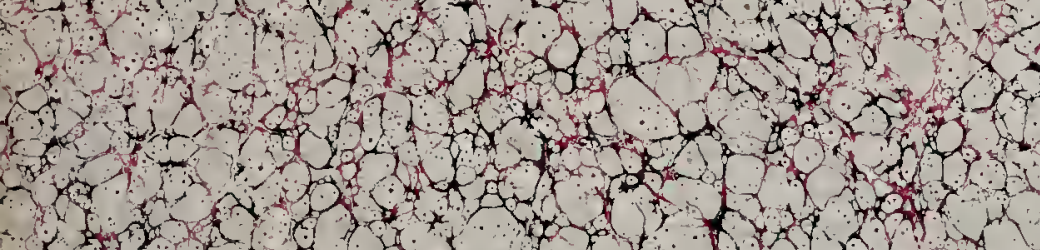
(1)

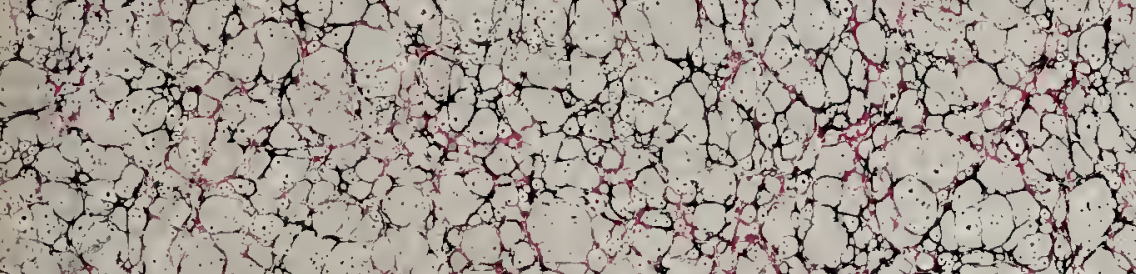

(-

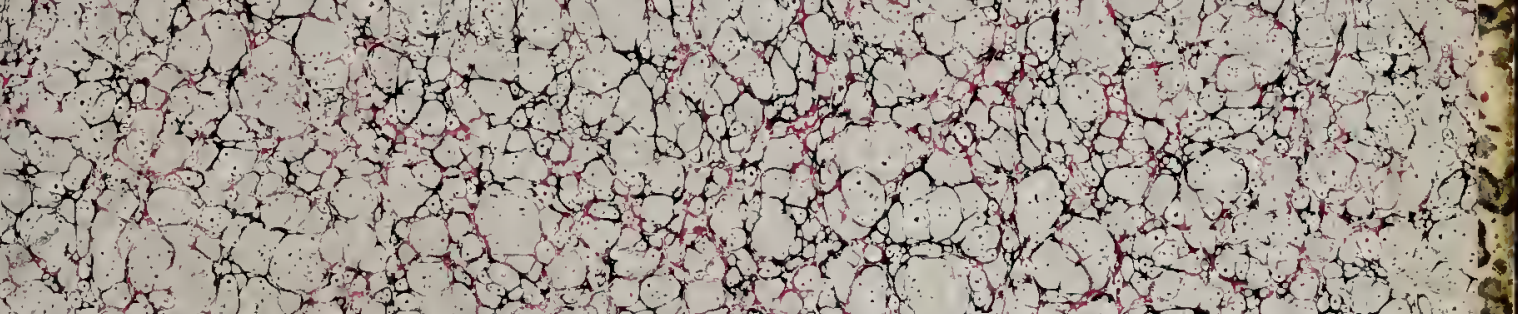

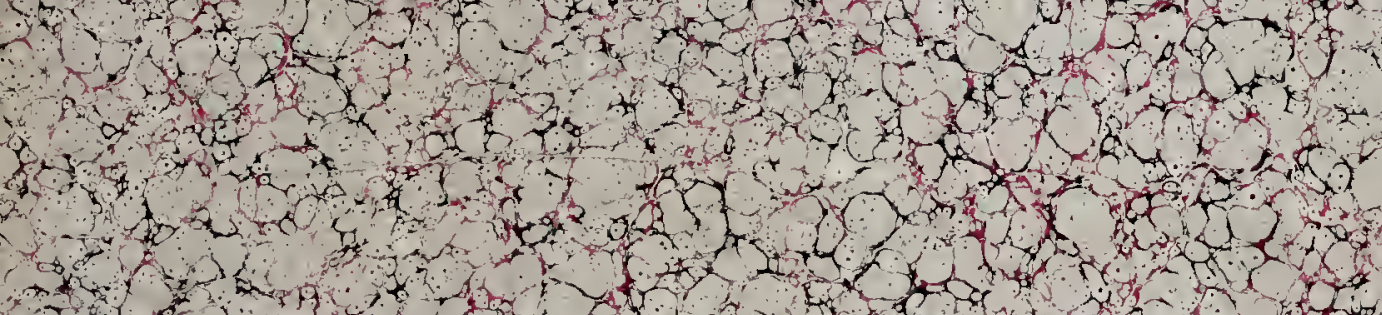

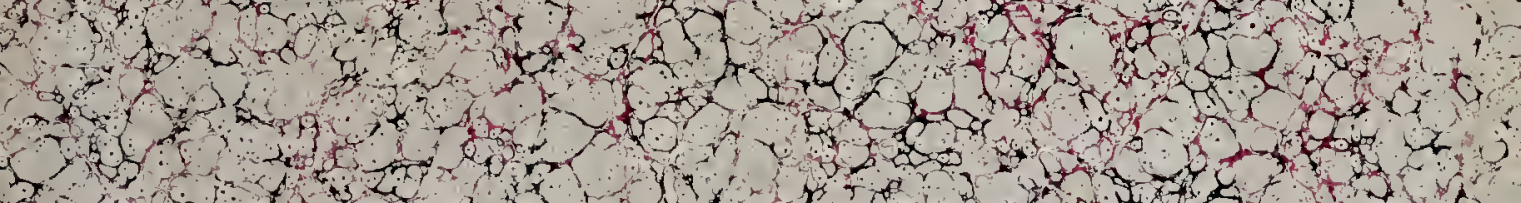

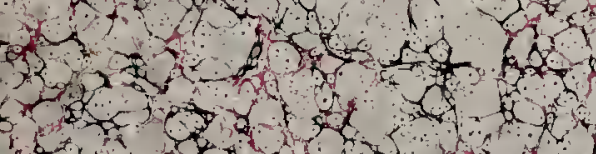

(5)

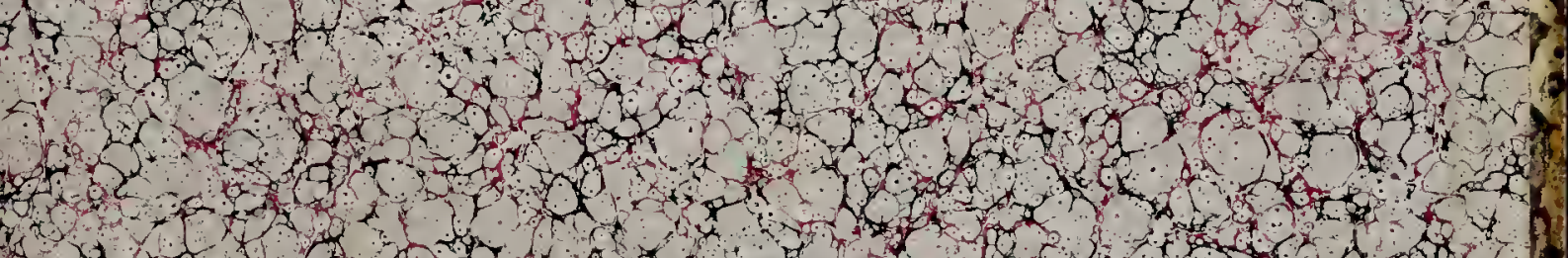

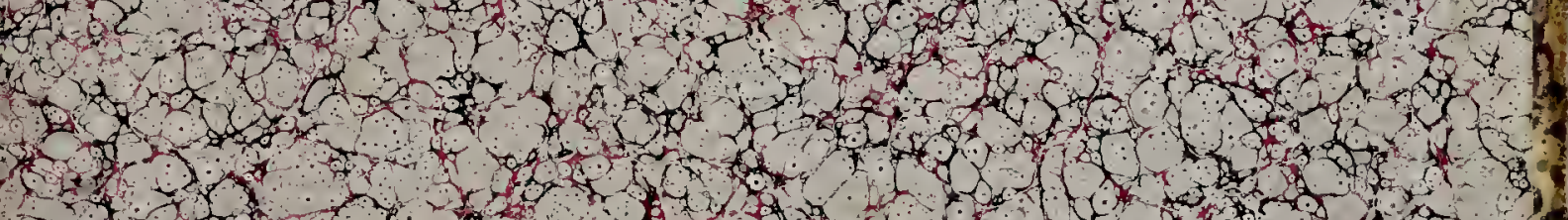

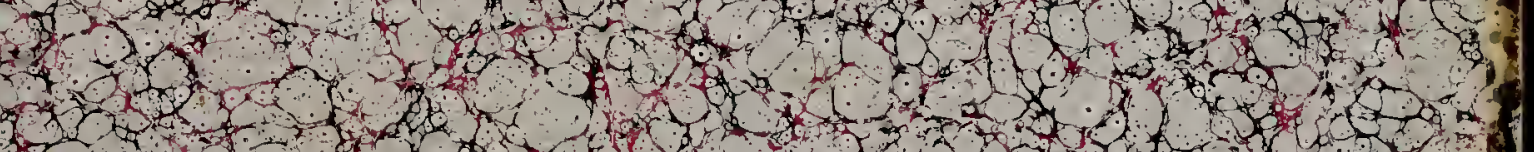

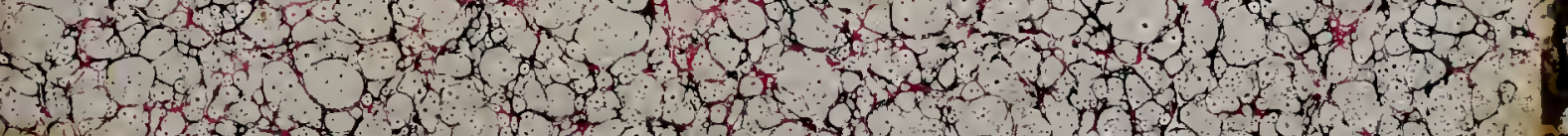

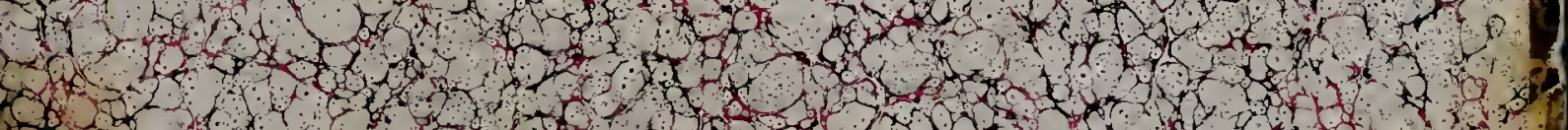

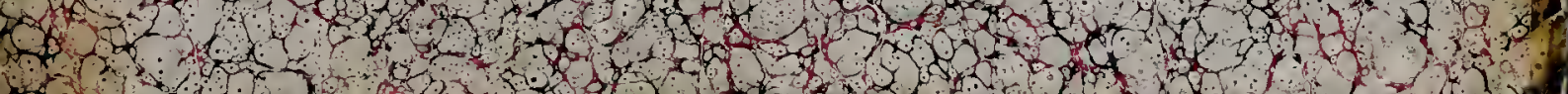

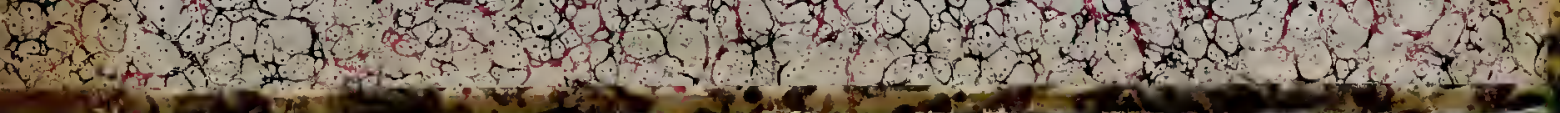




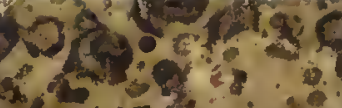

\section{- 0.6}

(

政

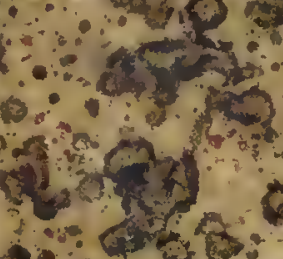

$0+2$

P.

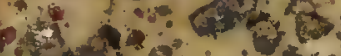

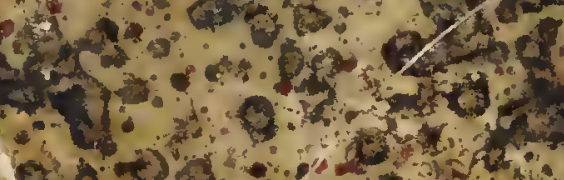

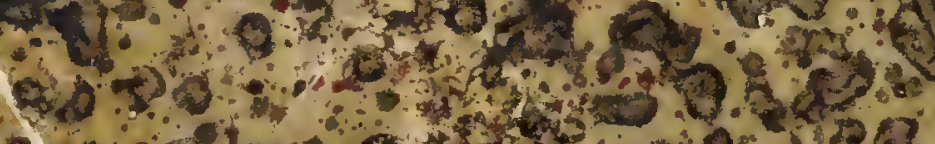

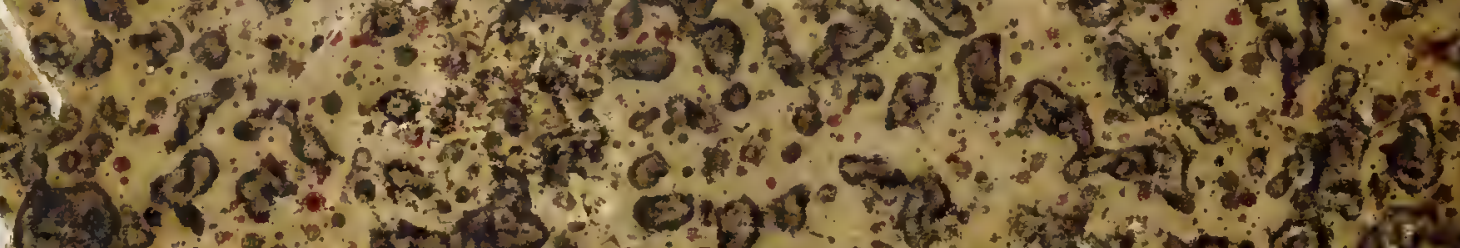

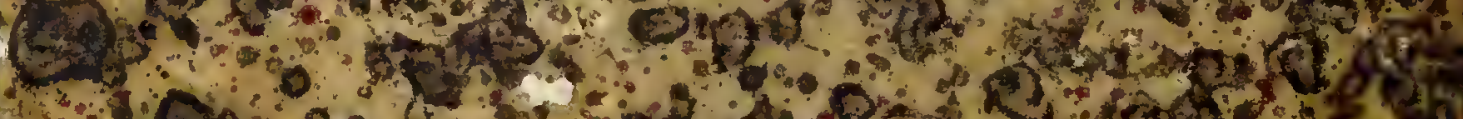

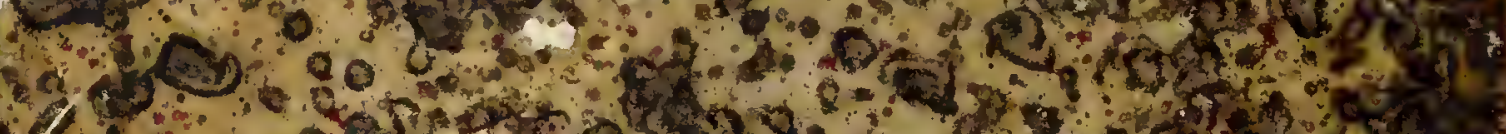
if $\rightarrow$ Fin 20,900

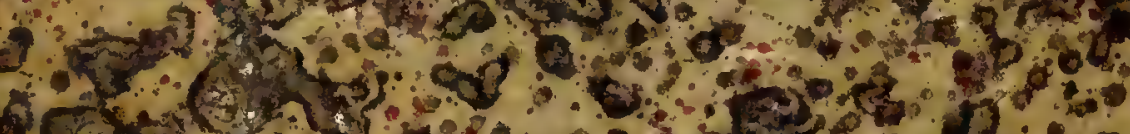

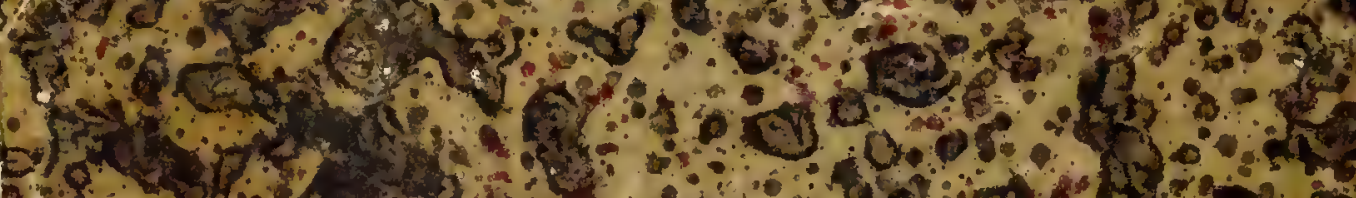

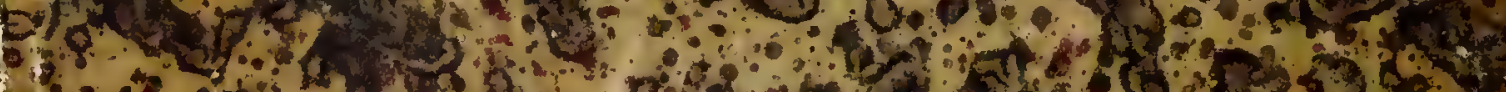

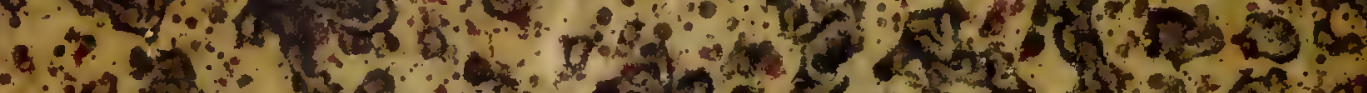

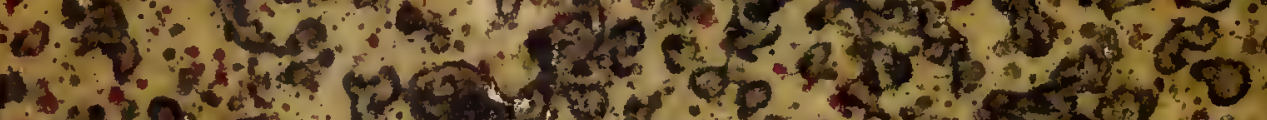

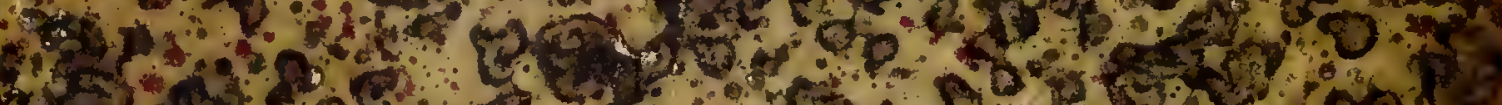

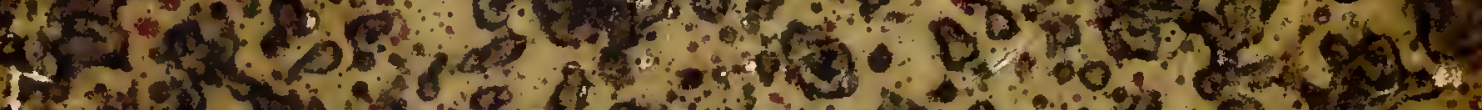

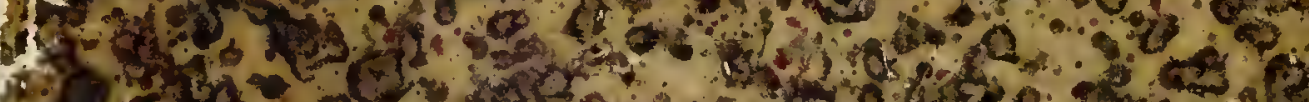
1
3

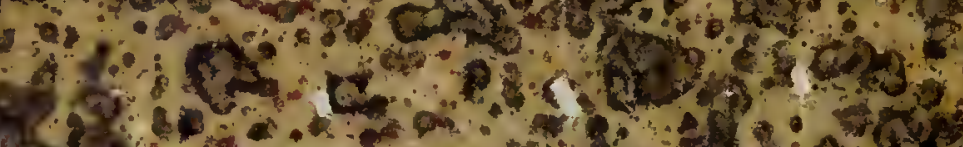

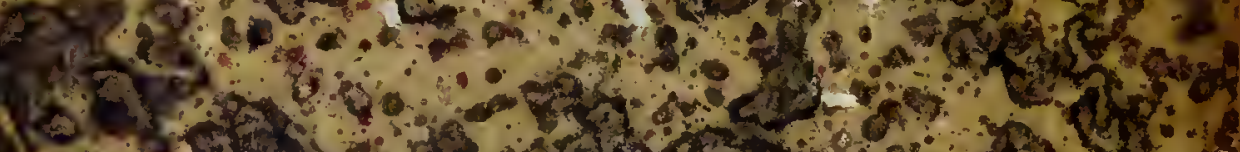

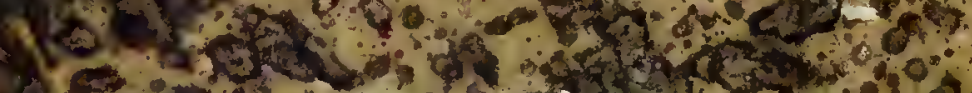
$x$ ar: 1. cos

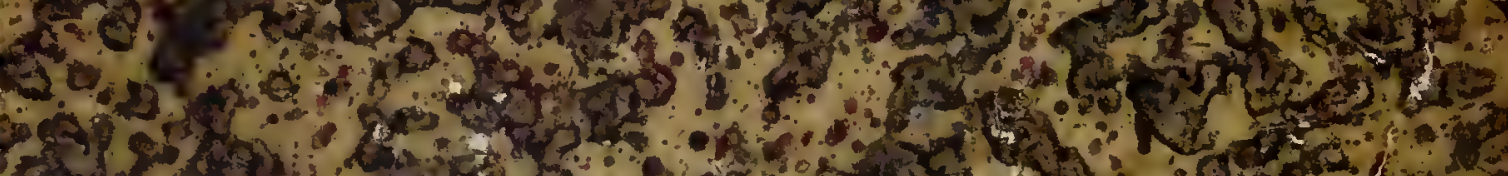

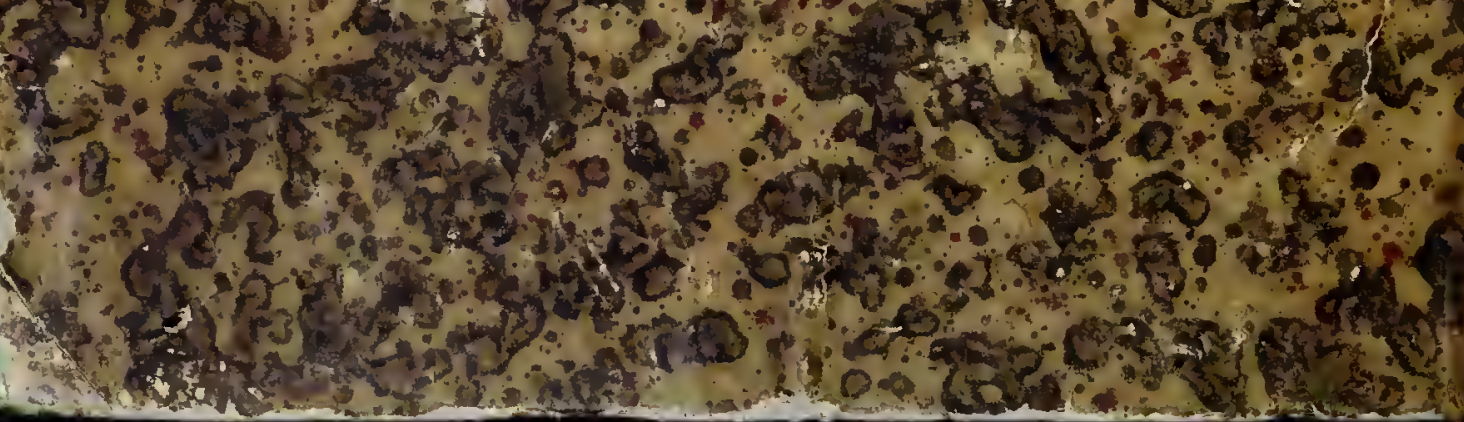

